Supporting Information

\title{
Chemoselective Installation of Diverse Succinimides on Fused Benzimidazoles via Rhodium-Catalyzed C-H Activation/Annulation: Chemosensor for Heavy Metals
}

\author{
Mohammad Aslam, ${ }^{\mathrm{a}}$ Sonaimuthu Mohandoss ${ }^{\mathrm{a}}$ and Yong Rok Lee*,a
}

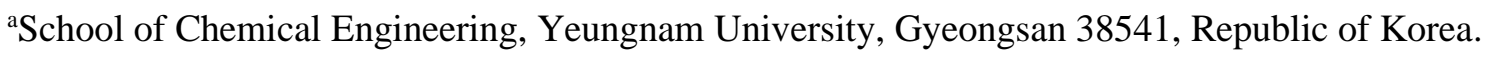

E-mail: yrlee@yu.ac.kr; Fax: +82-53-810-4631; Tel: +82-53-810-2529 


\section{Table of Contents}

$\begin{array}{lll}\text { General remarks } & \text { S3 }\end{array}$

$2 \quad$ General procedure for the synthesis of compounds $3 \quad$ S3

$3 \quad$ General procedure for the synthesis of compounds $4 \quad$ S4

$4 \quad$ Representative $1 \mathrm{mmol}$ scale synthesis of 3a $\quad$ S4

$5 \quad$ Representative $1 \mathrm{mmol}$ scale synthesis of $4 \mathrm{a} \quad \mathrm{S4}$

$6 \quad$ General procedure for the synthesis of 5a $\quad$ S5

$7 \quad$ General procedure for the synthesis of 3a from 5a $\quad$ S5

$8 \quad$ General procedure for the synthesis of 1a-D S5

9 General procedure for the synthesis of 3a-D S5

10 Control experiments $\quad$ S6

$\begin{array}{lll}11 & \text { Kinetic Isotopic Effect (KIE) study } & \text { S7 }\end{array}$

12 Characterization data for starting material 2o S12

13 Characterization data for synthesized compounds 3, 4 and $5 \quad$ S12

$14 \quad{ }^{1} \mathrm{H}$ NMR and ${ }^{13} \mathrm{C}$ NMR spectra of starting material $20 \quad$ S32

$15 \quad{ }^{1} \mathrm{H}$ NMR and ${ }^{13} \mathrm{C}$ NMR spectra of the synthesized compounds S33

16 Details of fluorophore analysis for heavy metal sensing S77

$\begin{array}{lll}17 & \text { References } & \mathbf{S 8 3}\end{array}$

18 X-ray crystallographic structure and data of compounds (3b and 4e) S86 


\section{General remarks}

All experiments were carried out under air atmosphere unless stated otherwise. Solvents were purchased from Sigma Aldrich and used without further purification. 2-Phenyl benzimidazole derivatives $\mathbf{1 a}$ and $\mathbf{1 m}$ were purchased from Thermo fisher. The starting materials $\mathbf{1 b}$-1k were prepared according to the literature procedures. ${ }^{1-5}$ The starting materials, maleimide derivatives 2a-2b, 2d-2o were purchased from Sigma Aldrich, Thermo fisher and TCI chemicals. The starting material $\mathbf{2 c}$ is a known compound prepared by literature method. ${ }^{6}$ The starting material 20 is a new compound and was prepared by literature method. ${ }^{6}$ The characterization data and NMR spectra for 20 is provided. Merck precoated silica gel plates (Art. 5554) treated with a fluorescent indicator were used for analytical thin-layer chromatography (TLC). Column chromatography was performed using silica gel 9385 (Merck) and ethyl acetate/hexane were used as eluents. Melting points are uncorrected and were determined using Fisher-Johns Melting Point Apparatus. ${ }^{1} \mathrm{H}$ NMR and ${ }^{13} \mathrm{C}$ NMR spectra were recorded on VNS (600 and 150 $\mathrm{MHz}$ ) spectrometer at the core research support centre for natural products and medical materials of Yeungnam University. The NMR spectra recorded in DMSO using $\delta=2.5$ and $39.5 \mathrm{ppm}$ and $\mathrm{CDCl}_{3}$ using $\delta=7.24$ and $77.0 \mathrm{ppm}$ as the solvent chemical shifts. All chemical shifts $(\delta)$ are expressed in units of ppm and $J$ values are given in Hz. Multiplicities are abbreviated as follows: $\mathrm{s}=$ singlet, $\mathrm{d}=$ doublet, $\mathrm{t}=$ triplet, $\mathrm{q}=$ quartet, $\mathrm{m}=$ multiplet or overlap of nonequivalent resonances, and $\mathrm{dd}=$ doublet of doublets. Infrared (IR) spectra were recorded on a PerkinElmer Spectrum Two ${ }^{\mathrm{TM}}$ IR spectrometer with frequencies expressed in $\mathrm{cm}^{-1}$, and high-resolution mass spectrometry (HRMS) was carried out using a JEOL JMS-700 spectrometer (Magnetic sector-Electric sector double focusing mass analyzer) at the Korea Basic Science Institute or by using Thermo Fisher Q exactive orbitrap mass spectrometer. The crystal structures of the compounds $\mathbf{3 b}$ and $\mathbf{4 e}$ were determined by single-crystal diffraction method at the Korea Basic Science Institute (KBSI, Western Seoul Center, Korea).

\section{General procedure for the synthesis of compounds 3}

An oven dried round bottom flask $(25 \mathrm{~mL})$ equipped with a magnetic stirrer was charged with 2-phenylbenzimidazole 1 (0.5 mmol), 1,2-DCE (3 mL), $\left[\mathrm{RhCp}^{*} \mathrm{Cl}_{2}\right]_{2}(1 \mathrm{~mol} \%), \operatorname{AgSbF}_{6}(20$ mol \%), acetic acid ( 2 equiv) and $N$-Phenyl maleimide 2 ( 2 equiv). The reaction mixture was stirred on oil bath for $12 \mathrm{~h}$ at $100{ }^{\circ} \mathrm{C}$. The progress of the reaction was monitored by TLC. After 
completion of the reaction, the volatiles were evaporated under reduced pressure. The crude product was purified over a column of silica gel and eluted with 75:25 hexane/ethyl acetate to afford the compounds 3 .

\section{General procedure for the synthesis of compounds 4}

An oven dried round bottom flask $(25 \mathrm{~mL})$ equipped with a magnetic stirrer was charged with 2-phenylbenzimidazole 1 ( $0.5 \mathrm{mmol})$, chlorobenzene (3 mL), $\left[\mathrm{RhCp}^{*} \mathrm{Cl}_{2}\right]_{2}(1 \mathrm{~mol} \%), \mathrm{AgSbF}_{6}$ (20 mol \%), NaOAc (1 equiv) and $N$-Phenyl maleimide 2 (1.2 equiv). The reaction mixture was stirred at oil bath for $12 \mathrm{~h}$ at $120^{\circ} \mathrm{C}$. The progress of the reaction was monitored by TLC. After completion of the reaction, the volatiles were evaporated under reduced pressure. The crude product was purified over a column of silica gel and eluted with 80:20 hexane/ethyl acetate to afford the compounds 4 .

\section{Representative $1 \mathrm{mmol}$ scale synthesis of $3 \mathrm{a}$}

An oven dried round bottom flask $(25 \mathrm{~mL})$ equipped with a magnetic stirrer was charged with 2-phenylbenzimidazole 1 ( $1 \mathrm{mmol}, 194 \mathrm{mg}$ ), 1,2-DCE (3 mL), [RhCp*Cl $]_{2}$ (1 mol \%), $\mathrm{AgSbF}_{6}$ (20 mol \%), acetic acid ( 2 equiv, $120 \mathrm{mg}$ ) and $N$-Phenyl maleimide 2 ( 2 equiv, $346 \mathrm{mg}$ ). The reaction mixture was stirred on an oil bath for $12 \mathrm{~h}$ at $100{ }^{\circ} \mathrm{C}$. The progress of the reaction was monitored by TLC. After completion of the reaction, the volatiles were evaporated under reduced pressure. The crude product was purified over a column of silica gel and eluted with 75:25 hexane/ethyl acetate to afford the compounds 3a in 84\% (455 mg) yield.

\section{Representative $1 \mathrm{mmol}$ scale synthesis of $4 \mathrm{a}$}

An oven dried round bottom flask $(25 \mathrm{~mL})$ equipped with a magnetic stirrer was charged with 2-phenylbenzimidazole 1 (1 mmol, $194 \mathrm{mg})$, chlorobenzene (3 $\mathrm{mL}),\left[\mathrm{RhCp}^{*} \mathrm{Cl}_{2}\right]_{2}(1 \mathrm{~mol} \%)$,

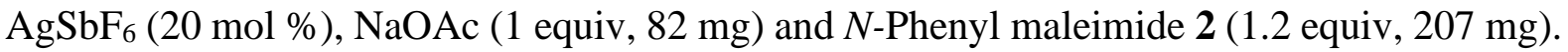
The reaction mixture was stirred at oil bath for $12 \mathrm{~h}$ at $120^{\circ} \mathrm{C}$. The progress of the reaction was monitored by TLC. After completion of the reaction, the volatiles were evaporated under reduced pressure. The crude product was purified over a column of silica gel and eluted with 80:20 hexane/ethyl acetate to afford the compounds $\mathbf{4 a}$ in $70 \%(255 \mathrm{mg}$ ) yield. 


\section{General procedure for the synthesis of $5 \mathrm{a}$}

An oven dried round bottom flask $(25 \mathrm{~mL})$ equipped with a magnetic stirrer was charged with 2-phenylbenzimidazole 1a $(0.5 \mathrm{mmol}, 97 \mathrm{mg}), \mathrm{DCM}(3 \mathrm{~mL}),\left[\mathrm{RhCp}^{*} \mathrm{Cl}_{2}\right]_{2}(1 \mathrm{~mol} \%), \mathrm{AgSbF}_{6}$ (20 mol \%), acetic acid (2 equiv, $60 \mathrm{mg}$ ) and $N$-Phenyl maleimide 2a (1 equiv, $87 \mathrm{mg}$ ). The reaction mixture was stirred at oil bath for $12 \mathrm{~h}$ at $50{ }^{\circ} \mathrm{C}$. The progress of the reaction was monitored by TLC. After completion of the reaction, the volatiles were evaporated under reduced pressure. The crude product was purified over a column of silica gel and eluted with 75:25 hexane/ethyl acetate to afford the compound 5a (80\%).

\section{General procedure for the synthesis of 3a from 5a}

An oven dried round bottom flask $(25 \mathrm{~mL})$ equipped with a magnetic stirrer was charged with 5a ( $0.2 \mathrm{mmol}, 82 \mathrm{mg}), \mathrm{DCE}(2 \mathrm{~mL})$, acetic acid (2 equiv) and $N$-Phenyl maleimide $2 \mathbf{a}$ ( 1 equiv). The mixture was stirred on an oil bath for $12 \mathrm{~h}$ at $100{ }^{\circ} \mathrm{C}$. After completion, the volatiles were evaporated under reduced pressure. The crude product was purified over a column of silica gel and eluted with 75:25 hexane/ethyl acetate to afford the compound 3a in 47\% (51 mg) yield.

\section{General procedure for the synthesis of 1a-D}

An oven dried round bottom flask $(25 \mathrm{~mL})$ equipped with a magnetic stirrer was charged with 2-phenylbenzimidazole 1a (1 mmol, $194 \mathrm{mg}), 1,2-\mathrm{DCE}$ and deuterated acetic acid (4 equiv, $240 \mathrm{mg}$ ). The reaction mixture was stirred at oil bath for $12 \mathrm{~h}$ at $100{ }^{\circ} \mathrm{C}$. After $12 \mathrm{~h}$, the volatiles were evaporated under reduced pressure. The crude product was purified over a column of silica gel and eluted with 75:25 hexane/ethyl acetate to afford the compound 1a-D (86\%).

\section{General procedure for the synthesis of 3a-D}

An oven dried round bottom flask $(25 \mathrm{~mL})$ equipped with a magnetic stirrer was charged with 2-phenylbenzimidazole 1a (1 mmol, $194 \mathrm{mg}), 1,2-\mathrm{DCE}(3 \mathrm{~mL}),\left[\mathrm{RhCp}^{*} \mathrm{Cl}_{2}\right]_{2}(1 \mathrm{~mol} \%)$, $\mathrm{AgSbF}_{6}(20 \mathrm{~mol} \%$ ), deuterated acetic acid (4 equiv, $240 \mathrm{mg}$ ) and $N$-Phenyl maleimide 2a (2 equiv, $346 \mathrm{mg}$ ). The reaction mixture was stirred at oil bath for $12 \mathrm{~h}$ at $100{ }^{\circ} \mathrm{C}$. The progress of the reaction was monitored by TLC. After completion of the reaction, the volatiles were evaporated under reduced pressure. The crude product was purified over a column of silica gel and eluted with 75:25 hexane/ethyl acetate to afford the compound 3a-D (84\%). 


\section{Control Experiments}

(A)

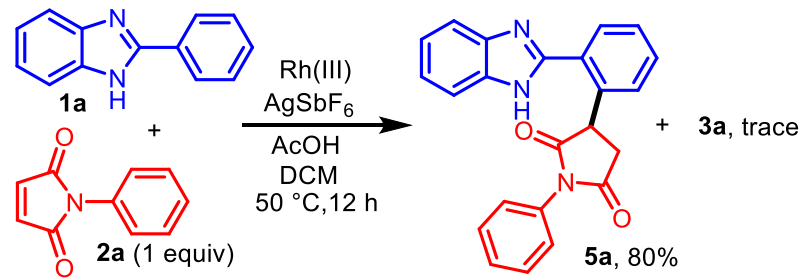

(B) $\quad \mathbf{5 a}+\underset{\text { (1 equiv) }}{\mathbf{2 a}} \stackrel{\mathrm{AcOH}(2 \text { equiv })}{\underset{\mathrm{DCE}}{\longrightarrow}}$

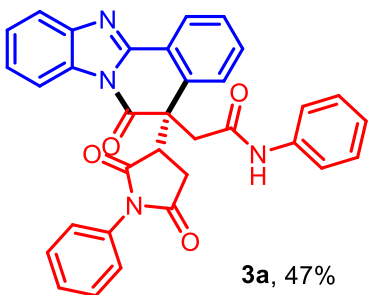

(C)
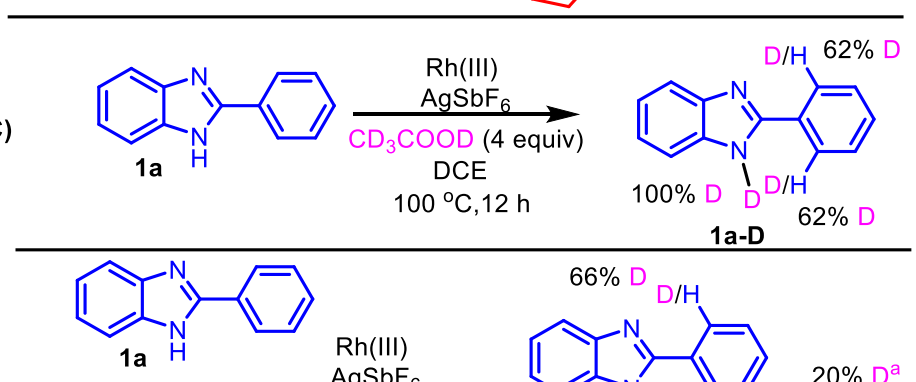

(D)

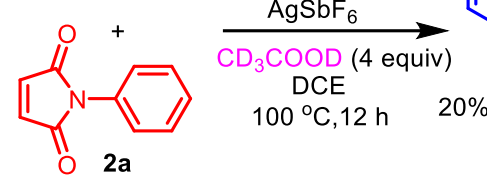
$66 \% \mathrm{D} D / H$

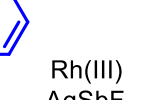

(E)

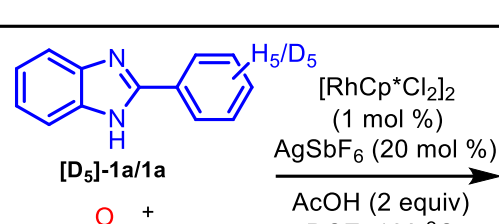<smiles>O=C1C=CC(=O)N1c1ccccc1</smiles>
DCE, $100^{\circ} \mathrm{C}$

intermolecular, $K I E=1.50$

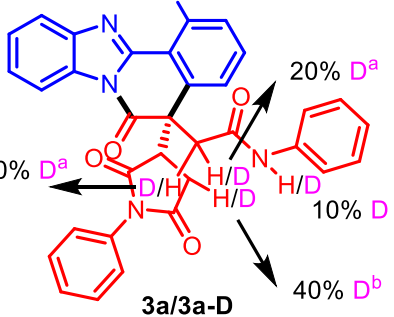

$3 a / 3 a-$
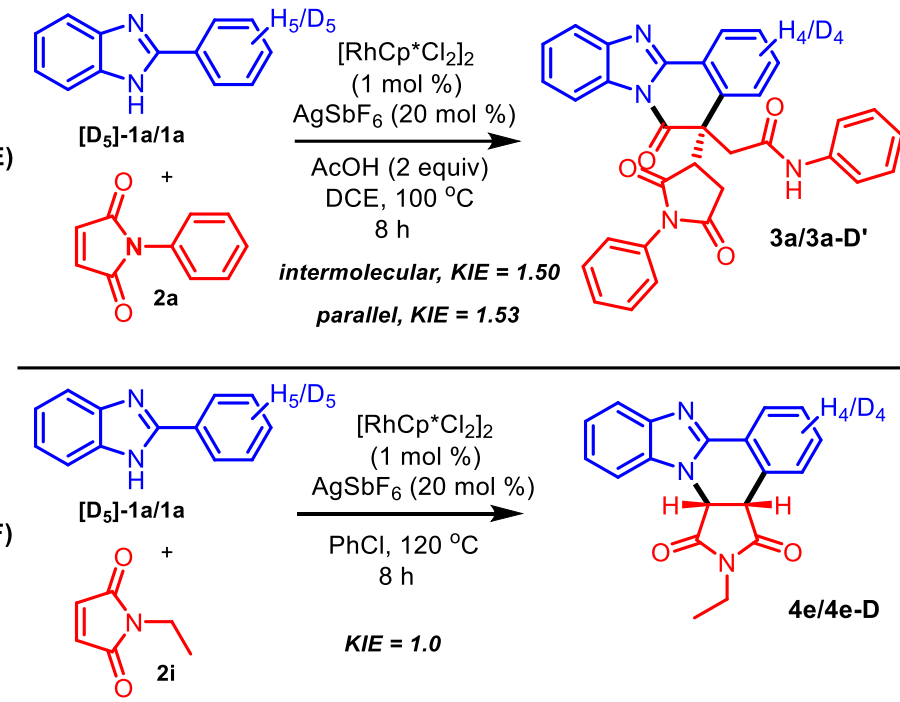

Scheme S1. Control Experiments 


\section{Kinetic Isotope Effect (KIE) Study (Intermolecular reaction experiment method)}

I. For reaction using acidic additive: An oven dried round bottom flask ( $25 \mathrm{~mL})$ equipped with a magnetic stirrer was charged with 2-phenylbenzimidazole 1a $(0.2 \mathrm{mmol}), 1,2-\mathrm{DCE}$ (2 $\mathrm{mL}),\left[\mathrm{RhCp}^{*} \mathrm{Cl}_{2}\right]_{2}(1 \mathrm{~mol} \%), \operatorname{AgSbF}_{6}(20 \mathrm{~mol} \%)$, acetic acid (2 equiv) and $N$-phenyl maleimide 2a (2 equiv). In a parallel reaction in another reaction flask, 2-(phenyl- $\left.d_{5}\right)-1 H$ benzo $[d]$ imidazole ([D5]-1a) $(0.2 \mathrm{mmol})$ was used instead of 1a. The reaction mixtures were stirred on an oil bath for $8 \mathrm{~h}$ at $100{ }^{\circ} \mathrm{C}$. Then, the reaction mixtures were combined, the volatiles were removed in vacuo and the product 3a/3a-D' was isolated by silica gel column chromatography $(\mathrm{Hex}:$ EtOAc $=75: 25)$ in $56 \%$ combined yield. The value of $K_{\mathrm{H}} / K_{\mathrm{D}}$ was calculated based on ${ }^{1} \mathrm{H}$ NMR. Here, $K_{\mathrm{H}} / K_{\mathrm{D}}=0.60 / 0.40=1.5$. HRMS was measured for 3a/3aD'. HRMS (ESI) $m / z,\left[\mathrm{M}+\mathrm{H}^{+}\right]$calcd for $\mathrm{C}_{33} \mathrm{H}_{21} \mathrm{D}_{4} \mathrm{~N}_{4} \mathrm{O}_{4}$ : 545.2121, Found: 545.2125.
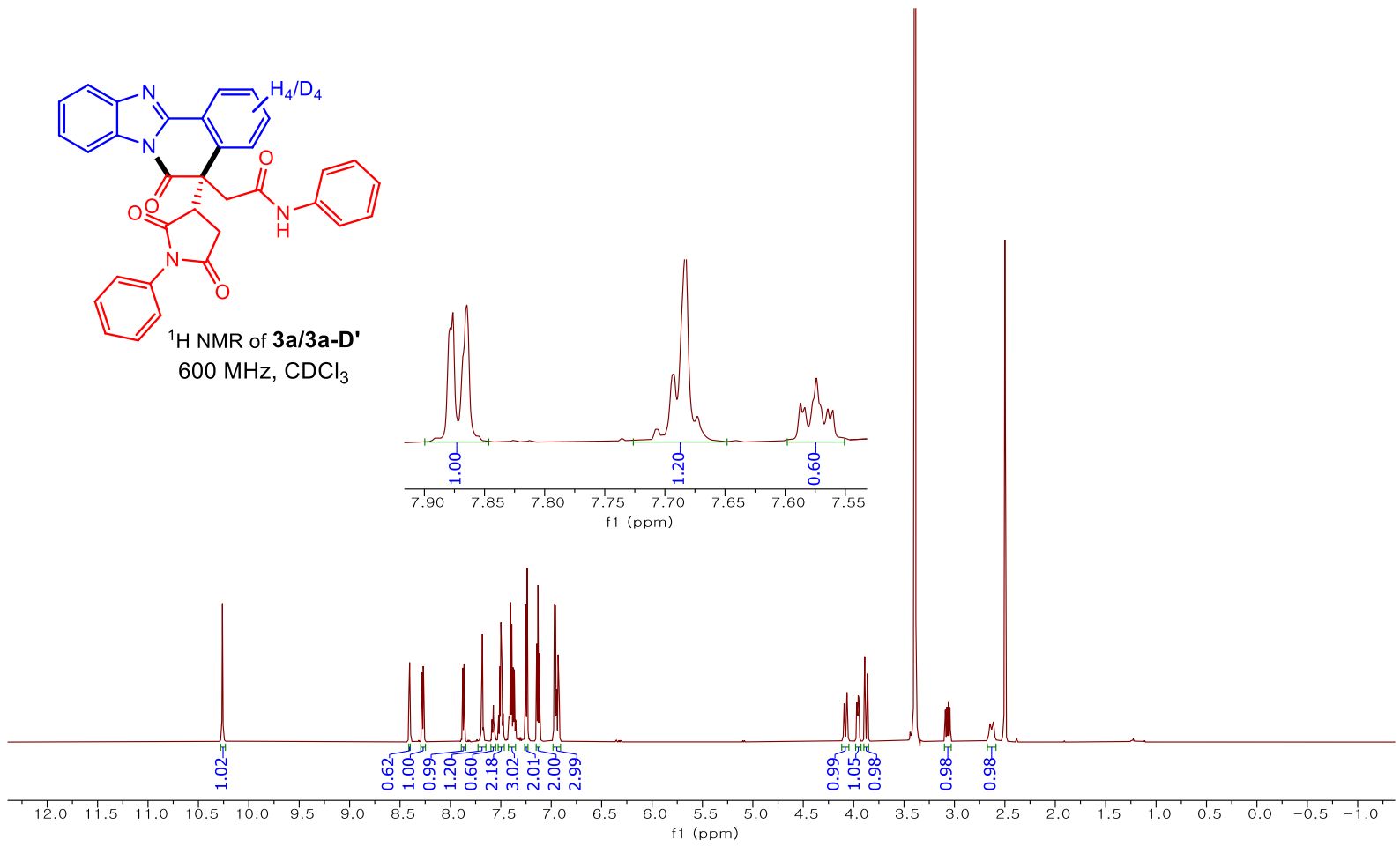

II. For reaction using basic additive: An oven dried round bottom flask (25 $\mathrm{mL}$ ) equipped with a magnetic stirrer was charged with 2-phenylbenzimidazole $\mathbf{1 a}(0.2 \mathrm{mmol})$, chlorobenzene (2 mL), $\left[\mathrm{RhCp}^{*} \mathrm{Cl}_{2}\right]_{2}(1 \mathrm{~mol} \%), \mathrm{AgSbF}_{6}(20 \mathrm{~mol} \%), \mathrm{NaOAc}(1$ equiv) and $N$-ethyl maleimide 
2i (1.2 equiv). In a parallel reaction in another reaction flask, 2-(phenyl- $\left.d_{5}\right)-1 H$ benzo $[d]$ imidazole ([D5]-1a) $(0.2 \mathrm{mmol})$ was used instead of 1a.The reaction mixtures were stirred on an oil bath for $8 \mathrm{~h}$ at $120^{\circ} \mathrm{C}$. Then, the reaction mixtures were combined, the volatiles were removed in vасио and the product 4e/4e-D was isolated by silica gel column chromatography $(\mathrm{Hex}:$ EtOAc $=75: 25)$ in $56 \%$ combined yield. The value of $K_{\mathrm{H}} / K_{\mathrm{D}}$ was calculated based on ${ }^{1} \mathrm{H}$ NMR. Here, $K_{\mathrm{H}} / K_{\mathrm{D}}=0.5 / 0.5=1.0$. HRMS was measured for 4e/4e-D. HRMS (ESI) $m / z$ [M+H'] calcd for $\mathrm{C}_{19} \mathrm{H}_{12} \mathrm{D}_{4} \mathrm{~N}_{3} \mathrm{O}_{2}: 322.1488$, Found: 322.1484 .

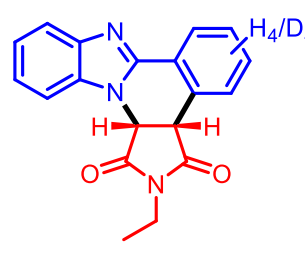

${ }^{1} \mathrm{H}$ NMR of $4 \mathrm{e} / 4 \mathrm{e}-\mathrm{D}$

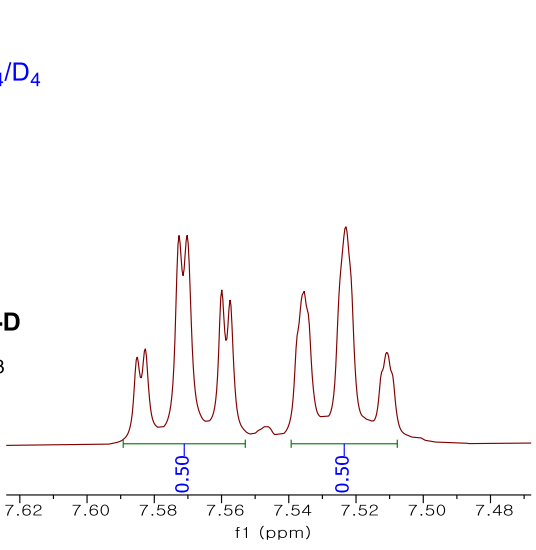
$600 \mathrm{MHz}^{\mathrm{CDCl}} \mathrm{CD}_{3}$

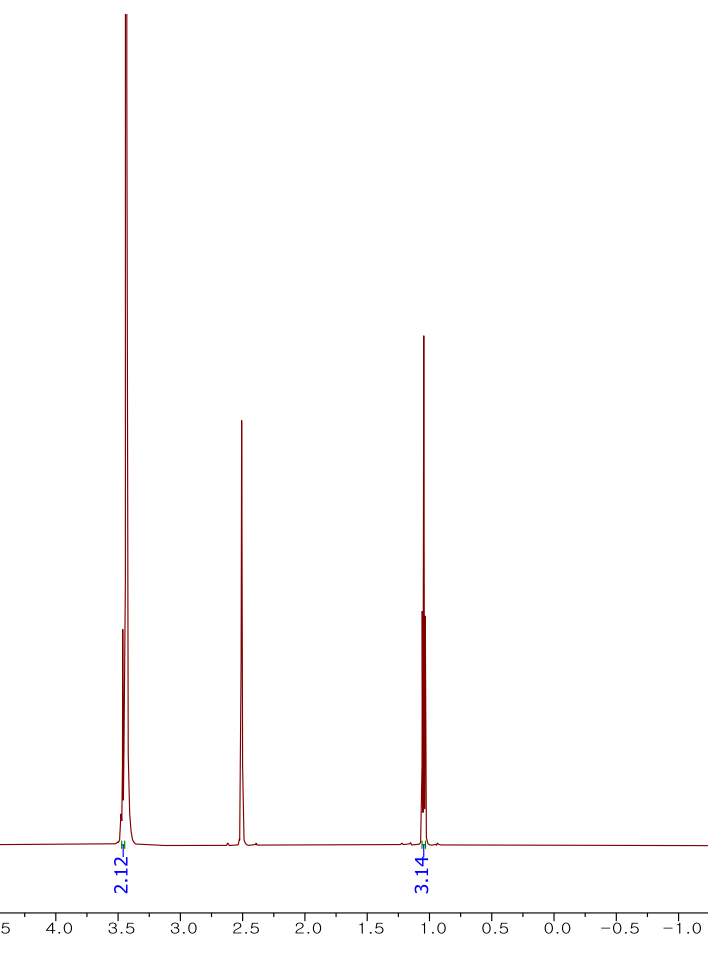

\section{Kinetic Isotope Effect (KIE) Study (Parallel reaction method):}

For reaction using acidic additive: An oven dried reaction vial equipped with a magnetic stirrer was charged with 2-phenylbenzimidazole $1 \mathbf{a}(0.1 \mathrm{mmol}), 1,2-\mathrm{DCE}(1 \mathrm{~mL}),\left[\mathrm{RhCp} * \mathrm{Cl}_{2}\right]_{2}$ (1 mol \%), $\operatorname{AgSbF}_{6}(20 \mathrm{~mol} \%)$, acetic acid (2 equiv) and $N$-phenyl maleimide 2a (2 equiv). The reaction mixtures were stirred on an oil bath for different time interval at $100{ }^{\circ} \mathrm{C}$. After given time, reaction mixture was filtered through syringe filter and solvent was evaporated and the product was isolated by silica gel column chromatography $(\mathrm{Hex}:$ EtOAc $=75: 25)$ to 
determine the conversion. These values were plotted as time vs conversion (\%) and linear fit was applied to generate graph.

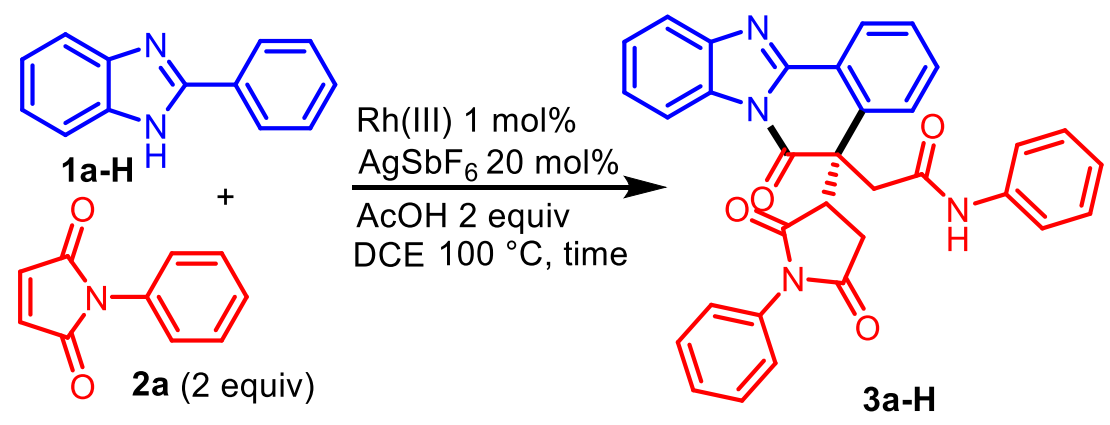

\begin{tabular}{|l|l|l|}
\hline entry & \multicolumn{1}{|c|}{$\begin{array}{c}\text { time } \\
\text { (min) }\end{array}$} & $\begin{array}{c}\text { conversion } \\
(\%)\end{array}$ \\
\hline 1 & 0 & 0 \\
\hline 2 & 30 & 5 \\
\hline 3 & 60 & 9 \\
\hline 4 & 90 & 11 \\
\hline 5 & 120 & 18 \\
\hline 6 & 180 & 24 \\
\hline 7 & 240 & 30 \\
\hline 8 & 300 & 36 \\
\hline 9 & 360 & 40 \\
\hline 10 & 420 & 46 \\
\hline 11 & 480 & 52 \\
\hline
\end{tabular}

For reaction using acidic additive: An oven dried reaction vial equipped with a magnetic stirrer was charged with 2-phenylbenzimidazole 1a-D (0.1 mmol), 1,2-DCE (1 mL), $\left[\mathrm{RhCp}^{*} \mathrm{Cl}_{2}\right]_{2}(1 \mathrm{~mol} \%), \operatorname{AgSbF}_{6}(20 \mathrm{~mol} \%)$, acetic acid (2 equiv) and $N$-phenyl maleimide 2a ( 2 equiv). The reaction mixtures were stirred on an oil bath for different time interval at 100 ${ }^{\circ} \mathrm{C}$. After given time, reaction mixture was filtered through syringe filter and solvent was evaporated and the product was isolated by silica gel column chromatography $(\mathrm{Hex}:$ EtOAc $=$ $75: 25)$ to determine the conversion. These values were plotted as time vs conversion (\%) and linear fit was applied to generate graph. 


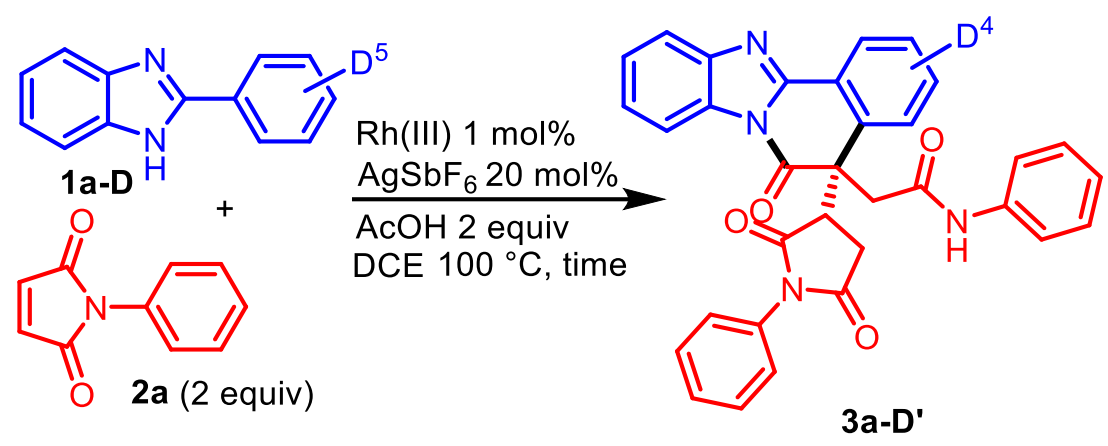

\begin{tabular}{|l|l|l|}
\hline entry & $\begin{array}{c}\text { time } \\
(\mathbf{m i n})\end{array}$ & $\begin{array}{c}\text { conversion } \\
(\mathbf{\%})\end{array}$ \\
\hline 1 & 0 & 0 \\
\hline 2 & 60 & 6 \\
\hline 3 & 120 & 12 \\
\hline 4 & 240 & 20 \\
\hline 5 & 360 & 26 \\
\hline 6 & 480 & 34 \\
\hline
\end{tabular}

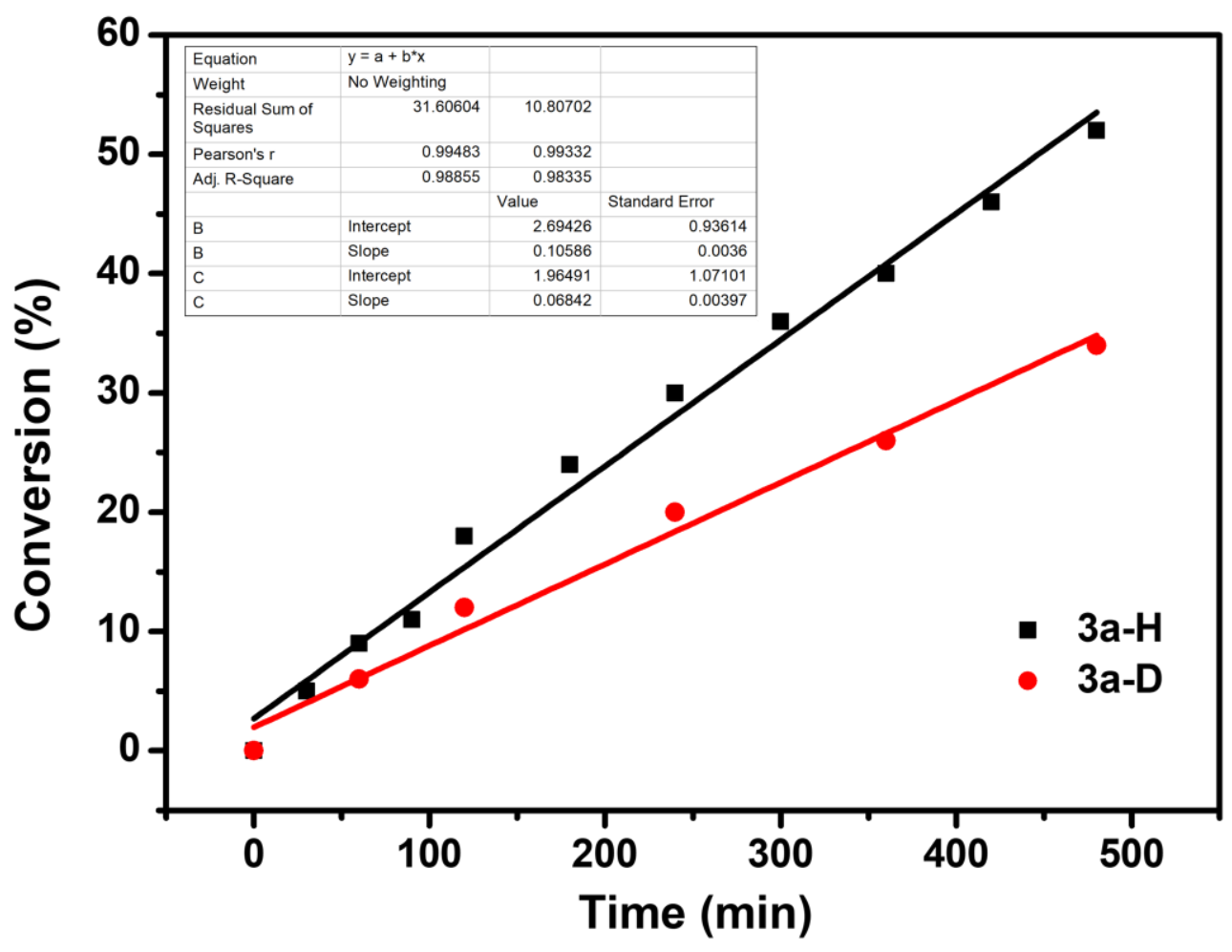

Fig S1. Time vs conversion. 


$$
\begin{gathered}
{[\text { Slope }]_{H}=\mathrm{k}_{\mathrm{H}}[0.1]} \\
0.105=\mathrm{k}_{\mathrm{H}}[0.1] \\
\text { SlopeD }=\mathrm{k}_{\mathrm{D}}[0.1] \\
0.0684=\mathrm{k}_{\mathrm{H}}[0.1] \\
\mathrm{k}_{\mathrm{H}} / \mathrm{k}_{\mathrm{D}}=1.53
\end{gathered}
$$




\section{Characterization data for starting material 2o}

\section{1-Undecyl-1H-pyrrole-2,5-dione (20):}<smiles>CC1CCCCC1=O</smiles>

The compound was prepared in $2 \mathrm{mmol}$ scale according to the literature procedure $^{6}$ and purified by column chromatography (EtOAc/hexane $\left.=5: 95\right)$. Yield: 65\% (327 mg); state: white solid; mp: 65-67 ${ }^{\circ} \mathrm{C} ; \quad{ }^{1} \mathrm{H}$ NMR (600 MHz, $\left.\mathrm{CDCl}_{3}\right) \delta 6.65(2 \mathrm{H}, \mathrm{s}), 3.47(2 \mathrm{H}, \mathrm{t}, J=7.8 \mathrm{~Hz}), 1.54(2 \mathrm{H}, \mathrm{p}, J=7.8 \mathrm{~Hz}), 1.27$ - $1.21(16 \mathrm{H}, \mathrm{m}), 0.84(3 \mathrm{H}, \mathrm{t}, J=7.2 \mathrm{~Hz}) ;{ }^{13} \mathrm{C} \mathrm{NMR}\left(150 \mathrm{MHz}, \mathrm{CDCl}_{3}\right) \delta 170.8,134.0,37.9$, 31.8, 29.5, 29.5, 29.4, 29.2, 29.1, 28.5, 26.7, 22.6, 14.0; IR (ATR): 3087, 2917, 2851, 1688, 1445, $1122 \mathrm{~cm}^{-1}$; HRMS (ESI) $m / z\left[\mathrm{M}+\mathrm{H}^{+}\right]$calcd for $\mathrm{C}_{15} \mathrm{H}_{26} \mathrm{NO}_{2}: 252.1958$, Found: 252.1956.

\section{Characterization data for synthesized compounds 3,4 and 5}

\section{2-(5-(2,5-Dioxo-1-phenylpyrrolidin-3-yl)-6-oxo-5,6-dihydrobenzo[4,5]imidazo[2,1-} a]isoquinolin-5-yl)- $N$-phenylacetamide (3a):

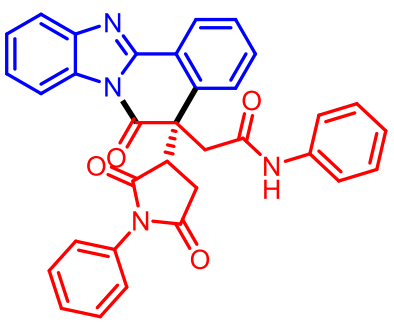

The compound was prepared according to the general procedure and purified by column chromatography $($ EtOAc/hexane $=25: 75)$. Yield: 86\% (233 mg); state: white solid; mp: $185-187{ }^{\circ} \mathrm{C}$; ${ }^{1} \mathrm{H}$ NMR (600 MHz, DMSO- $\left.d_{6}\right) \delta 10.28(1 \mathrm{H}, \mathrm{s}), 8.42(1 \mathrm{H}, \mathrm{d}, J=7.8 \mathrm{~Hz}), 8.29(1 \mathrm{H}$, $\mathrm{dd}, J=7.2,2.4 \mathrm{~Hz}), 7.88(1 \mathrm{H}, \mathrm{dd}, J=7.2,1.2 \mathrm{~Hz}), 7.70(2 \mathrm{H}, \mathrm{d}, J=4.2$ Hz), $7.57(1 \mathrm{H}, \mathrm{dd}, J=7.8,4.2 \mathrm{~Hz}), 7.52-7.47(2 \mathrm{H}, \mathrm{m}), 7.41(2 \mathrm{H}, \mathrm{t}, J=7.2 \mathrm{~Hz}), 7.37(1 \mathrm{H}, \mathrm{dd}$, $J=8.4,6.2 \mathrm{~Hz}), 7.27(2 \mathrm{H}, \mathrm{d}, J=7.8 \mathrm{~Hz}), 7.14(2 \mathrm{H}, \mathrm{t}, J=7.8 \mathrm{~Hz}), 6.98(2 \mathrm{H}, \mathrm{d}, J=7.8 \mathrm{~Hz})$, $6.94(1 \mathrm{H}, \mathrm{t}, J=7.2 \mathrm{~Hz}), 4.12(1 \mathrm{H}, \mathrm{d}, J=16.8 \mathrm{~Hz}), 3.98(1 \mathrm{H}, \mathrm{dd}, J=9.6,4.6 \mathrm{~Hz}), 3.90(1 \mathrm{H}, \mathrm{d}$, $J=16.8 \mathrm{~Hz}), 3.08(1 \mathrm{H}, \mathrm{dd}, J=18.6,9.6 \mathrm{~Hz}), 2.64(1 \mathrm{H}, \mathrm{d}, J=18 \mathrm{~Hz}) ;{ }^{13} \mathrm{C} \mathrm{NMR}(150 \mathrm{MHz}$, DMSO- $\left.d_{6}\right) \delta 175.4,173.8,170.7,167.5,149.5,143.4,138.2,137.4,131.8,131.7,130.8,128.9$, 128.5, 128.5, 126.6, 126.1, 125.9, 125.6, 125.5, 124.4, 123.4, 119.7, 119.0, 115.1, 50.8, 49.1, 44.4, 31.3; IR (ATR): 2963, 1715, 1258, 1014, $794 \mathrm{~cm}^{-1}$; HRMS $\left(\mathrm{EI}^{+}\right) \mathrm{m} / z\left[\mathrm{M}^{+}\right]$calcd for $\mathrm{C}_{33} \mathrm{H}_{24} \mathrm{~N}_{4} \mathrm{O}_{4}:$ 540.1798, Found: 540.1795. 
$N$-(2,6-Diethylphenyl)-2-(5-(1-(2,6-diethylphenyl)-2,5-dioxopyrrolidin-3-yl)-6-oxo-5,6dihydrobenzo[4,5]imidazo[2,1-a]isoquinolin-5-yl)acetamide (3b):

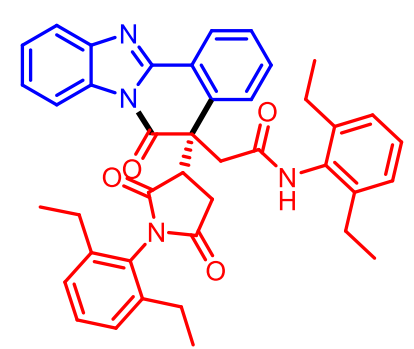

The compound was prepared according to the general procedure and purified by column chromatography $($ EtOAc/hexane $=$ 25:75). Yield: $86 \%$ (281 mg); state: white solid; mp: $210-212{ }^{\circ} \mathrm{C}$; ${ }^{1} \mathrm{H}$ NMR (600 MHz, DMSO- $\left.d_{6}\right) \delta 9.45(1 \mathrm{H}, \mathrm{s}), 8.35(1 \mathrm{H}, \mathrm{dd}, J=$ 7.8, $1.2 \mathrm{~Hz}), 8.22-8.20(1 \mathrm{H}, \mathrm{m}), 7.87(1 \mathrm{H}, \mathrm{d}, J=8.4 \mathrm{~Hz}), 7.77$ $-7.74(2 \mathrm{H}, \mathrm{m}), 7.59(1 \mathrm{H}, \mathrm{t}, J=7.8 \mathrm{~Hz}), 7.43-7.43(2 \mathrm{H}, \mathrm{m}), 7.25(1 \mathrm{H}, \mathrm{t}, J=7.8 \mathrm{~Hz}), 7.11$ $(1 \mathrm{H}, \mathrm{d}, J=6.0 \mathrm{~Hz}), 6.92-6.85(2 \mathrm{H}, \mathrm{m}), 4.50(1 \mathrm{H}, \mathrm{q}, J=9.0 \mathrm{~Hz}), 3.81-3.62(3 \mathrm{H}, \mathrm{m}), 3.39-$ $3.32(3 \mathrm{H}, \mathrm{m}), 2.38-2.37(2 \mathrm{H}, \mathrm{m}), 2.25-2.21(2 \mathrm{H}, \mathrm{m}), 2.03-1.97(1 \mathrm{H}, \mathrm{m}), 1.84-1.78(1 \mathrm{H}$, m), $1.50(2 \mathrm{H}, \mathrm{s}), 1.05-1.01(2 \mathrm{H}, \mathrm{m}), 0.95(3 \mathrm{H}, \mathrm{t}, J=7.8 \mathrm{~Hz}), 0.76(3 \mathrm{H}, \mathrm{t}, J=7.8 \mathrm{~Hz}), 0.68-$ $0.64(2 \mathrm{H}, \mathrm{m}) ;{ }^{13} \mathrm{C}$ NMR (150 MHz, DMSO- $\left.d_{6}\right) \delta 176.1,174.9,171.4,167.7,150.0,143.4$, $141.2,141.0,139.8,132.9,131.8,130.9,129.5,128.8,128.0,127.2,126.7,126.3,126.2$, 125.6, 125.2, 125.1, 124.5, 119.5, 115.1, 50.1, 49.1, 45.2, 32.3, 23.5, 23.3, 14.4, 13.9; IR (ATR): 1709, 1451, 1362, 1169, $747 \mathrm{~cm}^{-1}$; HRMS $\left(\mathrm{EI}^{+}\right) \mathrm{m} / z\left[\mathrm{M}^{+}\right]$calcd for $\mathrm{C}_{41} \mathrm{H}_{40} \mathrm{~N}_{4} \mathrm{O}_{4}$ : 652.3050, Found: 652.3047 .

$N$-(4-Methoxyphenyl)-2-(5-(1-(4-methoxyphenyl)-2,5-dioxopyrrolidin-3-yl)-6-oxo-5,6dihydrobenzo[4,5]imidazo[2,1-a]isoquinolin-5-yl)acetamide (3c):

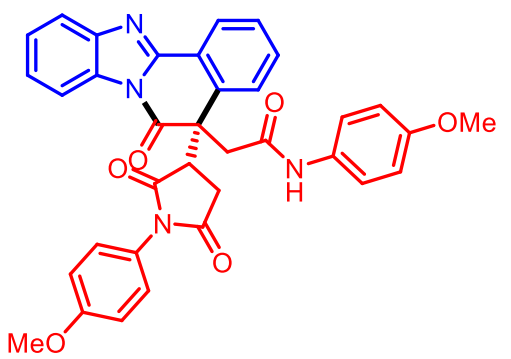

The compound was prepared according to the general procedure and purified by column chromatography $($ EtOAc/hexane $=25: 75)$. Yield: $84 \%(253 \mathrm{mg})$; state: white solid; mp: 200-202 ${ }^{\circ} \mathrm{C} ;{ }^{1} \mathrm{H}$ NMR (600 MHz, DMSO- $\left.d_{6}\right) \delta$ $10.10(1 \mathrm{H}, \mathrm{s}), 8.40(1 \mathrm{H}, \mathrm{d}, J=7.8 \mathrm{~Hz}), 8.27(1 \mathrm{H}, \mathrm{dd}, J=6.6$, $1.2 \mathrm{~Hz}), 7.87(1 \mathrm{H}, \mathrm{dd}, J=6.0,1.2 \mathrm{~Hz}), 7.70-7.64(2 \mathrm{H}, \mathrm{m})$, $7.59-7.55$ (1H, m), $7.52-7.46(2 \mathrm{H}, \mathrm{m}), 7.16-7.12(2 \mathrm{H}, \mathrm{m}), 6.94(2 \mathrm{H}, \mathrm{d}, J=9.0 \mathrm{~Hz}), 6.88$ $(2 \mathrm{H}, \mathrm{d}, J=9.0 \mathrm{~Hz}), 6.70(2 \mathrm{H}, \mathrm{d}, J=9.0 \mathrm{~Hz}), 4.04(1 \mathrm{H}, \mathrm{d}, J=16.8 \mathrm{~Hz}), 3.92(1 \mathrm{H}, \mathrm{dd}, J=9.6$, $4.2 \mathrm{~Hz}), 3.83(1 \mathrm{H}, \mathrm{d}, J=16.8 \mathrm{~Hz}), 3.74(3 \mathrm{H}, \mathrm{s}), 3.61(3 \mathrm{H}, \mathrm{s}), 3.03(1 \mathrm{H}, \mathrm{dd}, J=18.0,9.6 \mathrm{~Hz})$, $2.57(1 \mathrm{H}, \mathrm{d}, J=18.0 \mathrm{~Hz}) ;{ }^{13} \mathrm{C} \mathrm{NMR}\left(150 \mathrm{MHz}, \mathrm{DMSO}-d_{6}\right) \delta 175.7,174.1,170.8,166.9,159.0$, 155.2 , 149.6, 143.5, 137.4, 131.8, 131.3, 130.9, 128.5, 127.8, 126.1, 125.9, 125.6, 125.5, 124.4, 124.2, 120.6, 119.8, 115.1, 114.2, 113.7, 55.3, 55.0, 50.8, 49.0, 44.3, 31.2 ; IR (ATR): 3353, 
2925, 1697, 1366, $692 \mathrm{~cm}^{-1}$; HRMS (ESI) $m / z$ [ $\left[\mathrm{M}+\mathrm{H}^{+}\right]$calcd for $\mathrm{C}_{35} \mathrm{H}_{29} \mathrm{~N}_{4} \mathrm{O}_{6}$ : 601.2081, Found: 601.2077 .

$N$-(4-Fluorophenyl)-2-(5-(1-(4-fluorophenyl)-2,5-dioxopyrrolidin-3-yl)-6-oxo-5,6dihydrobenzo[4,5]imidazo[2,1-a]isoquinolin-5-yl)acetamide (3d):

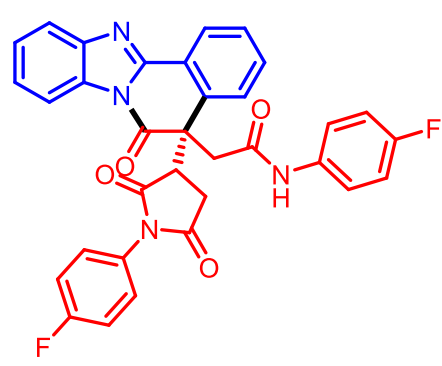

The compound was prepared according to the general procedure and purified by column chromatography (EtOAc/hexane = 25:75). Yield: $76 \%$ (220 mg); state: white solid; mp: 240-242 ${ }^{\circ} \mathrm{C} ;{ }^{1} \mathrm{H}$ NMR $\left(600 \mathrm{MHz}, \mathrm{DMSO}-d_{6}\right) \delta 10.34(1 \mathrm{H}, \mathrm{s}), 8.43(1 \mathrm{H}$, $\mathrm{d}, J=7.8 \mathrm{~Hz}), 8.29(1 \mathrm{H}, \mathrm{d}, J=7.8 \mathrm{~Hz}), 7.88(1 \mathrm{H}, \mathrm{d}, J=7.2 \mathrm{~Hz})$, $7.69(2 \mathrm{H}, \mathrm{d}, J=7.8 \mathrm{~Hz}), 7.61-7.55(1 \mathrm{H}, \mathrm{m}), 7.51-7.47(2 \mathrm{H}$, m), $7.31-7.24(4 \mathrm{H}, \mathrm{m}), 7.05(2 \mathrm{H}, \mathrm{dd}, J=8.4,4.2 \mathrm{~Hz}), 6.97(2 \mathrm{H}, \mathrm{t}, J=9.0 \mathrm{~Hz}), 4.10(1 \mathrm{H}, \mathrm{d}, J$ $=16.2 \mathrm{~Hz}), 3.98(1 \mathrm{H}, \mathrm{dd}, J=9.0,4.2 \mathrm{~Hz}), 3.89(1 \mathrm{H}, \mathrm{d}, J=16.8 \mathrm{~Hz}), 3.07(1 \mathrm{H}, \mathrm{dd}, J=18.0$, $9.0 \mathrm{~Hz}), 2.62(1 \mathrm{H}, \mathrm{d}, J=16.2 \mathrm{~Hz}) ;{ }^{13} \mathrm{C} \mathrm{NMR}\left(150 \mathrm{MHz}, \mathrm{DMSO}-d_{6}\right) \delta 175.4,173.8,170.7$, 167.4, 162.3 (C-F, $\left.{ }^{1} \mathrm{JC}-\mathrm{F}=244.5 \mathrm{~Hz}\right), 160.6\left(\mathrm{C}-\mathrm{F},{ }^{1} \mathrm{JC}-\mathrm{F}=244.5 \mathrm{~Hz}\right), 158.7\left(\mathrm{C}-\mathrm{F},{ }^{2} \mathrm{JC}-\mathrm{F}=\right.$ $238.3 \mathrm{~Hz}), 157.1\left(\mathrm{C}-\mathrm{F},{ }^{2} \mathrm{JC}-\mathrm{F}=238.3 \mathrm{~Hz}\right), 149.5,143.5,137.3,134.8,134.5,131.90,130.9$, $128.8\left(\mathrm{C}-\mathrm{F},{ }^{3} J \mathrm{C}-\mathrm{F}=9 \mathrm{~Hz}\right), 128.7\left(\mathrm{C}-\mathrm{F},{ }^{3} \mathrm{JC}-\mathrm{F}=9 \mathrm{~Hz}\right), 128.6,127.9,127.8,126.1\left(\mathrm{C}-\mathrm{F},{ }^{4} J \mathrm{C}-\mathrm{F}\right.$ $=8.2 \mathrm{~Hz}), 125.9\left(\mathrm{C}-\mathrm{F},{ }^{4} \mathrm{JC}-\mathrm{F}=8.2 \mathrm{~Hz}\right), 125.6\left(\mathrm{C}-\mathrm{F},{ }^{5} \mathrm{JC}-\mathrm{F}=8.2 \mathrm{~Hz}\right), 125.5\left(\mathrm{C}-\mathrm{F},{ }^{5} \mathrm{JC}-\mathrm{F}=8.2\right.$ $\mathrm{Hz}), 124.5,120.8\left(\mathrm{C}-\mathrm{F},{ }^{6} \mathrm{JC}-\mathrm{F}=8 \mathrm{~Hz}\right), 120.84\left(\mathrm{C}-\mathrm{F},{ }^{6} \mathrm{JC}-\mathrm{F}=8 \mathrm{~Hz}\right), 119.8,116.0$ (C-F, ${ }^{7} \mathrm{JC}-\mathrm{F}$ $=22.8 \mathrm{~Hz}), 115.9\left(\mathrm{C}-\mathrm{F},{ }^{7} \mathrm{JC}-\mathrm{F}=22.8 \mathrm{~Hz}\right), 115.2\left(\mathrm{C}-\mathrm{F},{ }^{8} \mathrm{JC}-\mathrm{F}=22.2 \mathrm{~Hz}\right), 115.1\left(\mathrm{C}-\mathrm{F},{ }^{8} \mathrm{JC}-\mathrm{F}=\right.$ $22.2 \mathrm{~Hz}), 115.1,50.8,49.1,44.3,31.3 ;{ }^{19} \mathrm{~F}$ NMR (565 Hz, DMSO- $\left.d_{6}\right) \delta-112.6(1 \mathrm{~F}),-119.0$ (1F). IR (ATR): 1712, 1600, 1492, 1359, $747 \mathrm{~cm}^{-1}$; HRMS $\left(\mathrm{EI}^{+}\right) \mathrm{m} / \mathrm{z}\left[\mathrm{M}^{+}\right]$calcd for $\mathrm{C}_{33} \mathrm{H}_{22} \mathrm{~F}_{2} \mathrm{~N}_{4} \mathrm{O}_{4}:$ 576.1609, Found: 576.1612.

\section{$N$-(4-Chlorophenyl)-2-(5-(1-(4-chlorophenyl)-2,5-dioxopyrrolidin-3-yl)-6-oxo-5,6-} dihydrobenzo[4,5]imidazo[2,1-a]isoquinolin-5-yl)acetamide (3e):

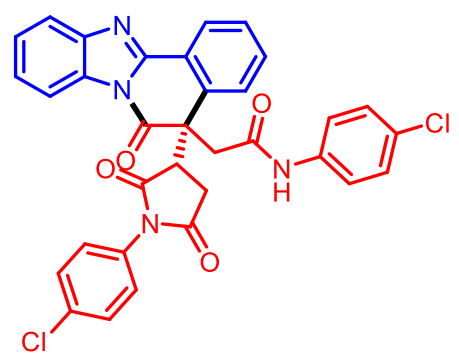

The compound was prepared according to the general procedure and purified by column chromatography $($ EtOAc/hexane $=25: 75)$. Yield: $77 \%(235 \mathrm{mg})$; state: white solid; mp: $173-175{ }^{\circ} \mathrm{C} ;{ }^{1} \mathrm{H}$ NMR $\left(600 \mathrm{MHz}, \mathrm{DMSO}-d_{6}\right) \delta$ $10.42(1 \mathrm{H}, \mathrm{s}), 8.41(1 \mathrm{H}, \mathrm{d}, J=7.2 \mathrm{~Hz}), 8.27(1 \mathrm{H}, \mathrm{d}, J=8.4$ $\mathrm{Hz}), 7.87(1 \mathrm{H}, \mathrm{d}, J=8.4 \mathrm{~Hz}), 7.70-7.64(2 \mathrm{H}, \mathrm{m}), 7.59-7.56$ 
$(1 \mathrm{H}, \mathrm{m}), 7.53-7.47(4 \mathrm{H}, \mathrm{m}), 7.29(2 \mathrm{H}, \mathrm{d}, J=9.0 \mathrm{~Hz}), 7.18(2 \mathrm{H}, \mathrm{d}, J=9.0 \mathrm{~Hz}), 7.03(2 \mathrm{H}, \mathrm{d}, J$ $=8.4 \mathrm{~Hz}), 4.09(1 \mathrm{H}, \mathrm{d}, J=16.8 \mathrm{~Hz}), 3.96(1 \mathrm{H}, \mathrm{dd}, J=9.0,4.2 \mathrm{~Hz}), 3.89(1 \mathrm{H}, \mathrm{d}, J=16.8 \mathrm{~Hz})$, $3.06(1 \mathrm{H}, \mathrm{dd}, J=18.0,9.0 \mathrm{~Hz}), 2.60(1 \mathrm{H}, \mathrm{d}, J=16.2 \mathrm{~Hz}) ;{ }^{13} \mathrm{C}$ NMR $\left(150 \mathrm{MHz}, \mathrm{DMSO}-d_{6}\right) \delta$ 175.3, 173.6, 170.7, 167.7, 149.5, 143.5, 137.1, 133.1, 131.9, 130.8, 130.4, 129.1, 128.6, 128.5, 128.3, 127.0, 126.0, 125.9, 125.6, 125.6, 124.4, 120.6, 119.8, 115.1, 50.7, 49.1, 44.4, 31.3. IR (ATR): 1714, 1492, 1359, 1172, $746 \mathrm{~cm}^{-1}$; HRMS $\left(\mathrm{EI}^{+}\right) \mathrm{m} / z\left[\mathrm{M}^{+}\right]$calcd for $\mathrm{C}_{33} \mathrm{H}_{22} \mathrm{Cl}_{2} \mathrm{~N}_{4} \mathrm{O}_{4}$ : 608.1018, Found: 608.1021.

\section{$N$-(4-Bromophenyl)-2-(5-(1-(4-bromophenyl)-2,5-dioxopyrrolidin-3-yl)-6-oxo-5,6-} dihydrobenzo[4,5]imidazo[2,1-a]isoquinolin-5-yl)acetamide (3f):

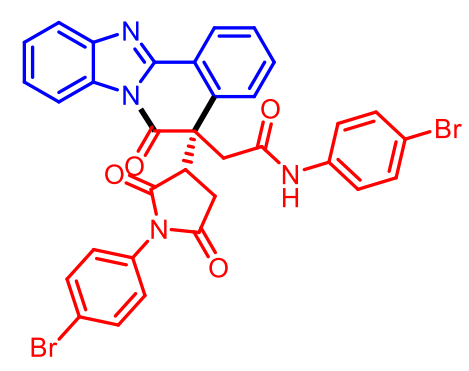

The compound was prepared according to the general procedure and purified by column chromatography (EtOAc/hexane $=$ 25:75). Yield: $74 \%$ (259 mg); state: orange solid; mp: 250-252 ${ }^{\circ} \mathrm{C} ;{ }^{1} \mathrm{H}$ NMR $\left(600 \mathrm{MHz}, \mathrm{DMSO}-d_{6}\right) \delta 10.42(1 \mathrm{H}, \mathrm{s}), 8.42(1 \mathrm{H}, \mathrm{d}$, $J=7.8 \mathrm{~Hz}), 8.27(1 \mathrm{H}, \mathrm{d}, J=7.2 \mathrm{~Hz}), 7.88(1 \mathrm{H}, \mathrm{d}, J=7.8 \mathrm{~Hz})$, $7.69-7.61(4 \mathrm{H}, \mathrm{m}), 7.58(1 \mathrm{H}, \mathrm{t}, J=7.2 \mathrm{~Hz}), 7.52-7.47(2 \mathrm{H}, \mathrm{m})$, $7.31(2 \mathrm{H}, \mathrm{d}, J=8.4 \mathrm{~Hz}), 7.25(2 \mathrm{H}, \mathrm{d}, J=9.0 \mathrm{~Hz}), 6.97(2 \mathrm{H}, \mathrm{d}, J=9 \mathrm{~Hz}), 4.10(1 \mathrm{H}, \mathrm{d}, J=16.8$ $\mathrm{Hz}), 3.96(1 \mathrm{H}, \mathrm{dd}, J=9.0,4.2 \mathrm{~Hz}), 3.89(1 \mathrm{H}, \mathrm{d}, J=16.8 \mathrm{~Hz}), 3.06(1 \mathrm{H}, \mathrm{dd}, J=18.0,12.6 \mathrm{~Hz})$, $2.60(1 \mathrm{H}, \mathrm{d}, J=17.4 \mathrm{~Hz}) ;{ }^{13} \mathrm{C}$ NMR $\left(150 \mathrm{MHz}, \mathrm{DMSO}-d_{6}\right) \delta 175.2,173.5,170.6,167.7,149.5$, $143.4,137.5,137.2,132.0,131.9,131.4,130.8,130.8,128.6,126.0,125.9,125.6,125.5,124.4$, 121.5, 120.9, 119.8, 115.1, 115.0, 50.7, 49.1, 44.4, 31.3. IR (ATR): 1710, 1487, 1360, 1190, 835, $748 \mathrm{~cm}^{-1}$; HRMS $\left(\mathrm{EI}^{+}\right) \mathrm{m} / z\left[\mathrm{M}^{+}\right]$calcd for $\mathrm{C}_{33} \mathrm{H}_{22} \mathrm{Br}_{2} \mathrm{~N}_{4} \mathrm{O}_{4}:$ 696.0008, Found: 696.0007 .

$N$-(4-Acetylphenyl)-2-(5-(1-(4-acetylphenyl)-2,5-dioxopyrrolidin-3-yl)-6-oxo-5,6dihydrobenzo[4,5]imidazo[2,1-a]isoquinolin-5-yl)acetamide (3g):

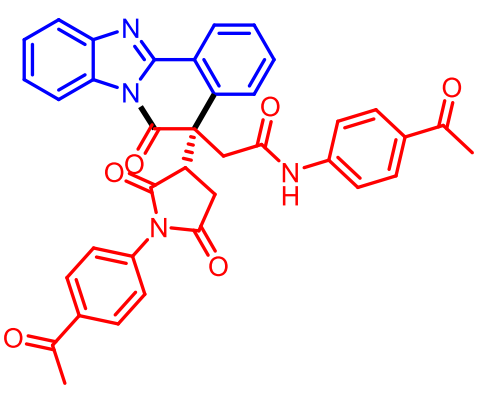

The compound was prepared according to the general procedure and purified by column chromatography $($ EtOAc/hexane $=25: 75)$. Yield: $70 \%(218 \mathrm{mg})$; state: orange solid; mp: $163-165{ }^{\circ} \mathrm{C} ;{ }^{1} \mathrm{H}$ NMR (600 MHz, DMSO- $\left.d_{6}\right) \delta 10.64$ $(1 \mathrm{H}, \mathrm{s}), 8.42(1 \mathrm{H}, \mathrm{d}, J=7.8 \mathrm{~Hz}), 8.27(1 \mathrm{H}, \mathrm{d}, J=7.2 \mathrm{~Hz}), 7.99$ $(2 \mathrm{H}, \mathrm{d}, J=8.4 \mathrm{~Hz}), 7.88(1 \mathrm{H}, \mathrm{d}, J=7.8 \mathrm{~Hz}), 7.76(2 \mathrm{H}, \mathrm{d}, J=$ $8.4 \mathrm{~Hz}), 7.69(2 \mathrm{H}, \mathrm{d}, J=3.6 \mathrm{~Hz}), 7.58(1 \mathrm{H}, \mathrm{dt}, J=8.1,4.1 \mathrm{~Hz}), 7.53-7.48(2 \mathrm{H}, \mathrm{m}) 7.41(2 \mathrm{H}$, d, $J=9.0 \mathrm{~Hz}), 7.16(2 \mathrm{H}, \mathrm{d}, J=7.8 \mathrm{~Hz}), 4.15(1 \mathrm{H}, \mathrm{d}, J=16.8 \mathrm{~Hz}), 4.00(1 \mathrm{H}, \mathrm{dd}, J=9.0,4.2$ 
$\mathrm{Hz}), 3.95(1 \mathrm{H}, \mathrm{d}, J=16.8 \mathrm{~Hz}), 3.10(1 \mathrm{H}, \mathrm{dd}, J=18.0,9.0 \mathrm{~Hz}), 2.65-2.60(1 \mathrm{H}, \mathrm{m}), 2.57(3 \mathrm{H}$, s), $2.43(3 \mathrm{H}, \mathrm{s}) ;{ }^{13} \mathrm{C}$ NMR (150 MHz, DMSO- $\left.d_{6}\right) \delta 197.1,196.3,175.1,173.5,170.6,168.2$, 149.5, 143.5, 142.4, 136.4, 135.5, 132.0, 131.8, 131.3, 130.8, 129.3, 128.9, 126.6, 126.1 , 126.0, 125.8, 125.7, 125.6, 124.4, 119.8, 118.3, 115.1, 50.7, 49.2, 44.5, 31.4, 26.7, 26.3; IR (ATR): 1713, 1600, 1497, 1388, $689 \mathrm{~cm}^{-1}$; HRMS $\left(\mathrm{EI}^{+}\right) \mathrm{m} / z\left[\mathrm{M}^{+}\right]$calcd for $\mathrm{C}_{37} \mathrm{H}_{28} \mathrm{~N}_{4} \mathrm{O}_{6}: 624.2009$, Found: 624.2013.

\section{2-(5-(2,5-Dioxo-1-(2,4,6-trichlorophenyl)pyrrolidin-3-yl)-6-oxo-5,6-dihydrobenzo[4,5]} imidazo[2,1-a]isoquinolin-5-yl)- $N$-(2,4,6-trichlorophenyl)acetamide (3h):

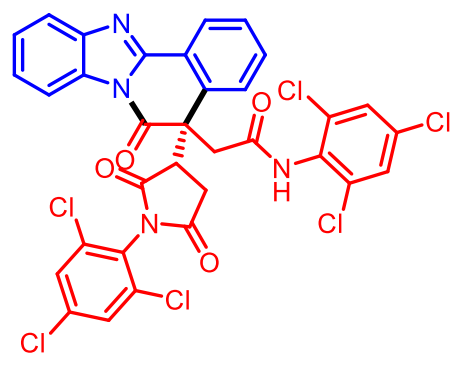

The compound was prepared according to the general procedure and purified by column chromatography (EtOAc/hexane $=25$ : 75). Yield: $74 \%$ (278 mg); state: white solid; mp: $>300{ }^{\circ} \mathrm{C} ;{ }^{1} \mathrm{H}$ NMR (600 MHz, DMSO- $\left.d_{6}\right) \delta 10.21(1 \mathrm{H}, \mathrm{s}), 8.33-8.30(1 \mathrm{H}, \mathrm{d}$, $9 \mathrm{~Hz}), 8.16(1 \mathrm{H}, \mathrm{dd}, J=7.2,1.8 \mathrm{~Hz}), 7.89-7.85(2 \mathrm{H}, \mathrm{m}), 7.80$ - $7.76(2 \mathrm{H}, \mathrm{m}), 7.73-7.69(1 \mathrm{H}, \mathrm{m}), 7.66-7.47$ (3H, m), 7.45$7.40(2 \mathrm{H}, \mathrm{m}), 4.53(1 \mathrm{H}, \mathrm{dd}, J=9.0,5.4 \mathrm{~Hz}), 3.80(2 \mathrm{H}, \mathrm{d}, J=15.6 \mathrm{~Hz}), 3.72(1 \mathrm{H}, \mathrm{d}, J=15.6$ $\mathrm{Hz}), 3.42(1 \mathrm{H}, \mathrm{dd}, J=18.0,9.0 \mathrm{~Hz}) ;{ }^{13} \mathrm{C} \mathrm{NMR}\left(150 \mathrm{MHz}, \mathrm{DMSO}-d_{6}\right) \delta 173.6,172.6,170.3$, $167.5,149.9,143.3,135.9,134.1,133.9,132.5,131.9,131.4,130.9,128.8,128.7,128.0,126.9$, 126.6, 125.7, 125.3, 125.0, 124.5, 119.6, 115.0, 49.8, 49.1, 44.4, 32.0. IR (ATR): 3373, 1723, 1452, 1175, $766 \mathrm{~cm}^{-1}$; HRMS $\left(\mathrm{EI}^{+}\right) \mathrm{m} / z\left[\mathrm{M}^{+}\right]$calcd for $\mathrm{C}_{33} \mathrm{H}_{18} \mathrm{Cl}_{6} \mathrm{~N}_{4} \mathrm{O}_{4}$ : 743.9459, Found: 743.9462 .

$N$-Ethyl-2-(5-(1-ethyl-2,5-dioxopyrrolidin-3-yl)-6-oxo-5,6-dihydrobenzo[4,5]imidazo[2,1a]isoquinolin-5-yl)acetamide (3i):

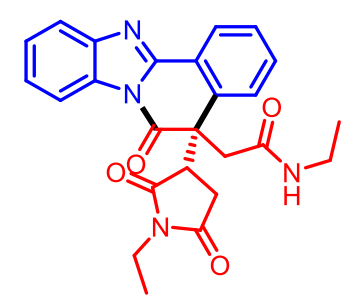

The compound was prepared according to the general procedure and purified by column chromatography $($ EtOAc/hexane $=25: 75)$. Yield: $73 \%$ (163 mg); state: white solid; mp: $215-217^{\circ} \mathrm{C} ;{ }^{1} \mathrm{H}$ NMR (600 MHz, $\left.\mathrm{CDCl}_{3}\right) \delta 8.58(1 \mathrm{H}, \mathrm{d}, J=7.8 \mathrm{~Hz}), 8.29(1 \mathrm{H}, \mathrm{d}, J=7.8 \mathrm{~Hz}), 7.81(1 \mathrm{H}$, $\mathrm{d}, J=7.8 \mathrm{~Hz}), 7.46(1 \mathrm{H}, \mathrm{t}, J=7.2 \mathrm{~Hz}), 7.41(3 \mathrm{H}, \mathrm{q}, J=7.2 \mathrm{~Hz}), 7.36$ $(1 \mathrm{H}, \mathrm{d}, J=7.8 \mathrm{~Hz}), 5.47(1 \mathrm{H}, \mathrm{s}), 4.03(1 \mathrm{H}, \mathrm{d}, J=16.2 \mathrm{~Hz}), 3.72(1 \mathrm{H}, \mathrm{d}, J=16.2 \mathrm{~Hz}), 3.54(1 \mathrm{H}$, dd, $J=9.6,5.4 \mathrm{~Hz}), 3.39-3.35(2 \mathrm{H}, \mathrm{m}), 2.95-2.90(2 \mathrm{H}, \mathrm{m}), 2.52(1 \mathrm{H}, \mathrm{dd}, J=18.2,9.2 \mathrm{~Hz})$, $2.03(1 \mathrm{H}, \mathrm{d}, J=14.4 \mathrm{~Hz}), 0.96(3 \mathrm{H}, \mathrm{t}, J=6.6 \mathrm{~Hz}), 0.84(3 \mathrm{H}, \mathrm{t}, J=7.2 \mathrm{~Hz}) ;{ }^{13} \mathrm{C} \mathrm{NMR}(150$ 
MHz, DMSO- $\left.d_{6}\right) \delta 176.1,174.7,170.8,167.9,149.7,143.4,138.0,131.5,130.8,128.1,126.2$, $125.7,125.3,125.1,124.4,119.6,115.0,50.4,48.7,43.8,33.1,32.8,31.0,14.2$, 12.3. IR (ATR): 1700, 1600, 1497, 1267, $832 \mathrm{~cm}^{-1}$; HRMS (ESI) $\mathrm{m} / z\left[\mathrm{M}+\mathrm{H}^{+}\right]$calcd for $\mathrm{C}_{25} \mathrm{H}_{25} \mathrm{~N}_{4} \mathrm{O}_{4}$ : 445.1870, Found: 445.1867.

\section{$N$-Cyclohexyl-2-(5-(1-cyclohexyl-2,5-dioxopyrrolidin-3-yl)-6-oxo-5,6-dihydrobenzo[4,5]} imidazo[2,1-a]isoquinolin-5-yl)acetamide (3j):

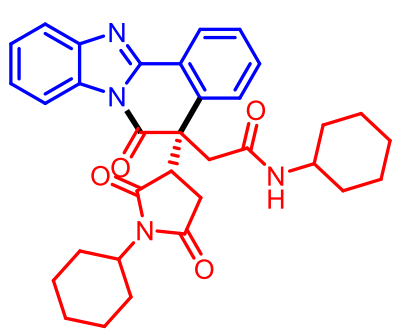

The compound was prepared according to the general procedure and purified by column chromatography (EtOAc/hexane $=25: 75)$. Yield: 80\% (221 mg); state: white solid; mp: 205-207 ${ }^{\circ} \mathrm{C} ;{ }^{1} \mathrm{H}$ NMR $\left(600 \mathrm{MHz}, \mathrm{DMSO}-d_{6}\right) \delta 8.34(1 \mathrm{H}, \mathrm{d}, J=8.4 \mathrm{~Hz}), 8.18(1 \mathrm{H}, \mathrm{d}, J=$ $7.2 \mathrm{~Hz}), 7.93(1 \mathrm{H}, \mathrm{d}, J=8.4 \mathrm{~Hz}), 7.83(1 \mathrm{H}, \mathrm{d}, J=8.4 \mathrm{~Hz}), 7.59(1 \mathrm{H}$, t, $J=6.0 \mathrm{~Hz}), 7.53(2 \mathrm{H}, \mathrm{t}, J=6.6 \mathrm{~Hz}), 7.49-7.41(2 \mathrm{H}, \mathrm{m}), 3.70(1 \mathrm{H}, \mathrm{dd}, J=9.0,4.2 \mathrm{~Hz}), 3.65$ $-3.58(2 \mathrm{H}, \mathrm{m}), 3.43(1 \mathrm{H}, \mathrm{d}, J=15.6 \mathrm{~Hz}), 3.04(1 \mathrm{H}, \mathrm{s}), 2.82(1 \mathrm{H}, \mathrm{dd}, J=18.0,9.2 \mathrm{~Hz}), 2.55$ $(1 \mathrm{H}, \mathrm{s}), 1.77(1 \mathrm{H}, \mathrm{d}, J=9.4 \mathrm{~Hz}), 1.74-1.66(1 \mathrm{H}, \mathrm{m}), 1.64-1.47(5 \mathrm{H}, \mathrm{m}), 1.40(2 \mathrm{H}, \mathrm{s}), 1.23$ $(2 \mathrm{H}, \mathrm{dd}, J=28.2,11.6 \mathrm{~Hz}), 1.07(4 \mathrm{H}, \mathrm{dd}, J=30.0,13.7 \mathrm{~Hz}), 1.00-0.91(4 \mathrm{H}, \mathrm{m}), 0.74-0.66$ $(1 \mathrm{H}, \mathrm{m}) ;{ }^{13} \mathrm{C}$ NMR $\left(150 \mathrm{MHz}, \mathrm{DMSO}-d_{6}\right) \delta 176.3,174.9,170.8,167.2,149.7,143.4,131.4$, 130.9, 128.0, 126.4, 125.6, 125.3, 125.0, 124.3, 119.5, 115.0, 50.6, 47.1, 43.9, 31.8, 30.9, 28.0, 27.8, 25.1, 24.9, 24.6, 24.2, 24.0. IR (ATR): 2931, 2857, 1596, 1555, $1363 \mathrm{~cm}^{-1}$; HRMS (EI $\left.{ }^{+}\right)$ $m / z\left[\mathrm{M}^{+}\right]$calcd for $\mathrm{C}_{33} \mathrm{H}_{36} \mathrm{~N}_{4} \mathrm{O}_{4}:$ 552.2737, Found: 552.2734.

\section{$N$-(4-(2,5-Dioxo-2,5-dihydro-1H-pyrrol-1-yl)phenyl)-2-(5-(1-(4-(2,5-dioxo-2,5-dihydro-} 1H-pyrrol-1-yl)phenyl)-2,5-dioxopyrrolidin-3-yl)-6-oxo-5,6-dihydrobenzo[4,5]imidazo $[2,1-a]$ isoquinolin-5-yl)acetamide (3k):

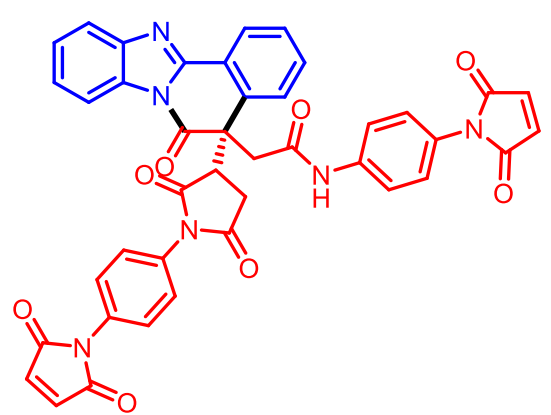

The compound was prepared according to the general procedure and purified by column chromatography (EtOAc/hexane = 30:70). Yield: 80\% (293 mg); state: off white solid; mp: $180-182{ }^{\circ} \mathrm{C}$; ${ }^{1} \mathrm{H}$ NMR (600 MHz, DMSO$\left.d_{6}\right) \delta 10.46(1 \mathrm{H}, \mathrm{s}), 8.41(1 \mathrm{H}, \mathrm{d}, J=8.4 \mathrm{~Hz}), 8.30-8.27$ $(1 \mathrm{H}, \mathrm{m}), 7.88(1 \mathrm{H}, \mathrm{d}, J=9.0 \mathrm{~Hz}), 7.71(2 \mathrm{H}, \mathrm{d}, J=7.8 \mathrm{~Hz})$, $7.58(1 \mathrm{H}, \mathrm{t}, J=8.4 \mathrm{~Hz}), 7.53-7.47(2 \mathrm{H}, \mathrm{m}), 7.40(2 \mathrm{H}, \mathrm{d}, J=8.4 \mathrm{~Hz}), 7.36(2 \mathrm{H}, \mathrm{d}, J=9.0 \mathrm{~Hz})$, $7.19(2 \mathrm{H}, \mathrm{s}), 7.09(6 \mathrm{H}, \mathrm{t}, J=4.4 \mathrm{~Hz}), 4.13(1 \mathrm{H}, \mathrm{d}, J=16.8 \mathrm{~Hz}), 3.98(1 \mathrm{H}, \mathrm{dd}, J=9.6,4.8 \mathrm{~Hz})$, 
$3.92(1 \mathrm{H}, \mathrm{d}, J=16.2 \mathrm{~Hz}), 3.08(1 \mathrm{H}, \mathrm{dd}, J=18.0,9.6 \mathrm{~Hz}), 2.60(1 \mathrm{H}, \mathrm{d}, J=14.2 \mathrm{~Hz}) ;{ }^{13} \mathrm{C} \mathrm{NMR}$ $\left(150 \mathrm{MHz}, \mathrm{DMSO}-d_{6}\right) \delta 175.4,173.8,170.7,169.9,169.8,169.6,167.7,149.5,143.5,137.6$, 134.7, 134.5, 132.0, 131.6, 130.9, 130.7, 128.7, 127.2, 127.1, 126.5, 126.1, 125.9, 125.6, 125.5, 124.4, 119.8, 119.2, 115.1, 50.8, 49.1, 44.4, 31.3. IR (ATR): 1709, 1514, 1359, 1022, $770 \mathrm{~cm}^{-}$ ${ }^{1}$; HRMS $\left(\mathrm{FAB}^{+}\right) \mathrm{m} / z\left[\mathrm{M}+\mathrm{H}^{+}\right]$calcd for $\mathrm{C}_{41} \mathrm{H}_{27} \mathrm{~N}_{6} \mathrm{O}_{8}$ : 731.1890, Found: 731.1894 .

\section{$N$-(4-(4-(2,5-Dioxo-2,5-dihydro-1H-pyrrol-1-yl)benzyl)phenyl)-2-(5-(1-(4-(4-(2,5-dioxo-}

\section{2,5-dihydro-1H-pyrrol-1-yl)benzyl)phenyl)-2,5-dioxopyrrolidin-3-yl)-6-oxo-5,6-}

dihydrobenzo[4,5]imidazo[2,1-a]isoquinolin-5-yl)acetamide (31):

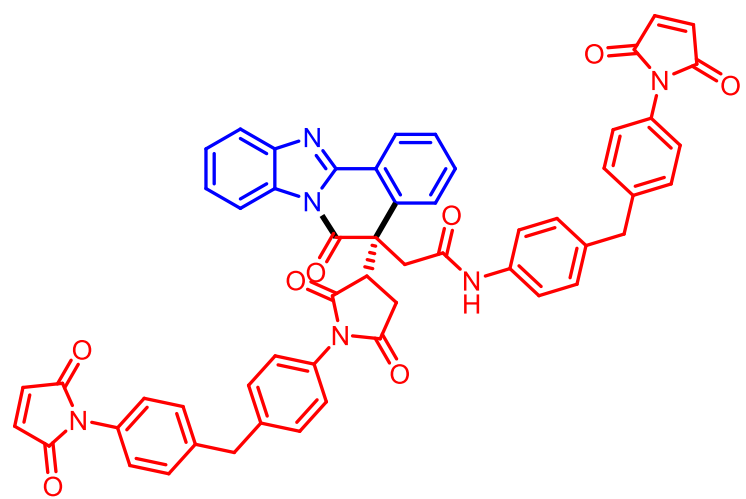

The compound was prepared according to the general procedure and purified by column chromatography (EtOAc/hexane $=70: 30)$. Yield: 68\% (310 mg); state: off white solid; mp: 195-197 ${ }^{\circ} \mathrm{C} ;{ }^{1} \mathrm{H}$ NMR (600 MHz, DMSO- $\left.d_{6}\right) \delta$ $10.23(1 \mathrm{H}, \mathrm{s}), 8.39(1 \mathrm{H}, \mathrm{dd}, J=7.8,1.2 \mathrm{~Hz}), 8.26$ $(1 \mathrm{H}, \mathrm{dd}, J=7.8,1.2 \mathrm{~Hz}), 7.87(1 \mathrm{H}, \mathrm{dd}, J=7.8$, $1.8 \mathrm{~Hz}), 7.78-7.76(1 \mathrm{H}, \mathrm{m}), 7.69-7.66(1 \mathrm{H}, \mathrm{m}), 7.65-7.62(1 \mathrm{H}, \mathrm{m}), 7.55(1 \mathrm{H}, \mathrm{dt}, J=7.2$, $1.2 \mathrm{~Hz}), 7.51-7.46(1 \mathrm{H}, \mathrm{m}), 7.37-7.35(2 \mathrm{H}, \mathrm{m}), 7.32-7.30(2 \mathrm{H}, \mathrm{m}), 7.28-7.26(2 \mathrm{H}, \mathrm{m})$, $7.25-7.23(2 \mathrm{H}, \mathrm{m}), 7.21-7.18(2 \mathrm{H}, \mathrm{m}), 7.17-7.16(2 \mathrm{H}, \mathrm{m}), 7.16-7.15(4 \mathrm{H}, \mathrm{m}), 7.01(2 \mathrm{H}$, d, $J=9.0 \mathrm{~Hz}), 6.90(2 \mathrm{H}, \mathrm{d}, J=8.4 \mathrm{~Hz}), 4.08-4.01(3 \mathrm{H}, \mathrm{m}), 3.98(1 \mathrm{H}, \mathrm{s}), 3.93-3.91(1 \mathrm{H}, \mathrm{m})$, $3.86-3.82(2 \mathrm{H}, \mathrm{m}), 3.05-3.01(1 \mathrm{H}, \mathrm{m}), 2.56-2.50(1 \mathrm{H}, \mathrm{m}) .{ }^{13} \mathrm{C}$ NMR $(150 \mathrm{MHz}, \mathrm{DMSO}-$ $\left.d_{6}\right) \delta 175.5,173.9,173.1,172.7,170.7,169.9,169.9,167.4,149.5,143.5,141.5,141.1,140.6$, 140.4, 136.4, 135.9, 134.6, 134.6, 130.9, 129.7, 129.6, 129.5, 129.3, 129.3, 129.2, 129.2 129.1, 129.0, 128.9, 128.9, 126.9, 126.7, 126.6, 126.1, 125.9, 125.6, 125.5, 124.4, 119.8, 119.2, 115.1, 50.8, 49.0, 44.4, 40.1, 40.0, 31.3. IR (ATR): 1705, 1511, 1377, 1147, $755 \mathrm{~cm}^{-1}$; $\left.\mathrm{HRMS}_{(\mathrm{FAB}}{ }^{+}\right)$ $m / z,\left[\mathrm{M}+\mathrm{H}^{+}\right]$calcd for $\mathrm{C}_{55} \mathrm{H}_{39} \mathrm{~N}_{6} \mathrm{O}_{8}$ : 911.2829, Found: 911.2831. 


\section{2-(5-(2,5-Dioxo-1-phenylpyrrolidin-3-yl)-9,10-dimethyl-6-oxo-5,6-dihydrobenzo[4,5]}

imidazo[2,1-a]isoquinolin-5-yl)- $N$-phenylacetamide (3m):

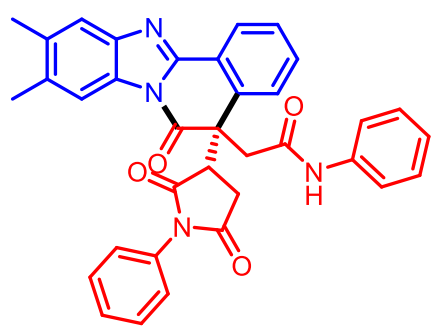

The compound was prepared according to the general procedure and purified by column chromatography $($ EtOAc/hexane $=$ 25:75). Yield: $78 \%$ (222 mg); state: white solid; mp: $220-222{ }^{\circ} \mathrm{C}$; ${ }^{1} \mathrm{H}$ NMR (600 MHz, DMSO-d $) \delta 10.24(1 \mathrm{H}, \mathrm{s}), 8.36(1 \mathrm{H}, \mathrm{d}, \mathrm{J}=$ $7.8 \mathrm{~Hz}), 8.06(1 \mathrm{H}, \mathrm{s}), 7.65-7.62(3 \mathrm{H}, \mathrm{m}), 7.57-7.53(1 \mathrm{H}, \mathrm{m})$, $7.43(2 \mathrm{H}, \mathrm{t}, \mathrm{J}=7.2 \mathrm{~Hz}), 7.38(1 \mathrm{H}, \mathrm{t}, \mathrm{J}=7.2 \mathrm{~Hz}), 7.25(2 \mathrm{H}, \mathrm{d}, \mathrm{J}=8.4 \mathrm{~Hz}), 7.13(2 \mathrm{H}, \mathrm{t}, \mathrm{J}=7.2$ Hz), $6.99(2 \mathrm{H}, \mathrm{d}, \mathrm{J}=7.2 \mathrm{~Hz}), 6.93(1 \mathrm{H}, \mathrm{t}, \mathrm{J}=7.2 \mathrm{~Hz}), 4.10(1 \mathrm{H}, \mathrm{d}, \mathrm{J}=16.8 \mathrm{~Hz}), 3.91(1 \mathrm{H}, \mathrm{dd}$, $\mathrm{J}=10.8,5.4 \mathrm{~Hz}), 3.84(1 \mathrm{H}, \mathrm{d}, \mathrm{J}=16.8 \mathrm{~Hz}), 3.02(1 \mathrm{H}, \mathrm{dd}, \mathrm{J}=18.0,9.6 \mathrm{~Hz}), 2.52-2.51(1 \mathrm{H}, \mathrm{m})$, $2.40(3 \mathrm{H}, \mathrm{s}), 2.39(3 \mathrm{H}, \mathrm{s}) ;{ }^{13} \mathrm{C}$ NMR $\left(150 \mathrm{MHz}, \mathrm{DMSO}-d_{6}\right) \delta 175.5,173.8,170.5,167.5,148.7$, $141.9,138.9,138.2$, 134.6, 134.5, 131.7, 131.5, 129.2 , 129.0, 128.6, 128.5, 126.6, 126.0, 125.3, 124.7, 123.3, 119.8, 118.9, 115.3, 50.7, 49.0, 44.3, 31.2, 20.1, 19.8.; IR (ATR): 1709, 1514, 1359, 1154, $821 \mathrm{~cm}^{-1}$; HRMS $\left(\mathrm{EI}^{+}\right) \mathrm{m} / z\left[\mathrm{M}^{+}\right]$calcd for $\mathrm{C}_{35} \mathrm{H}_{28} \mathrm{~N}_{4} \mathrm{O}_{4}$ : 568.2111, Found: 568.2110

\section{2-(9,10-Bichloro-5-(2,5-dioxo-1-phenylpyrrolidin-3-yl)-6-oxo-5,6-dihydrobenzo[4,5]} imidazo[2,1-a]isoquinolin-5-yl)- $N$-phenylacetamide (3n):

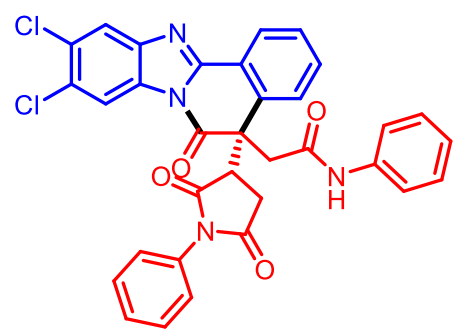

The compound was prepared according to the general procedure and purified by column chromatography $($ EtOAc/hexane $=$ 25:75). Yield: $75 \%$ (229 mg); state: white solid; $\mathrm{mp}: 280-282$ ${ }^{\circ} \mathrm{C},{ }^{1} \mathrm{H}$ NMR (600 MHz, DMSO-d6) $\delta 10.27(1 \mathrm{H}, \mathrm{s}), 8.42(1 \mathrm{H}$, s), $8.40(1 \mathrm{H}, \mathrm{d}, J=7.8 \mathrm{~Hz}), 8.22(1 \mathrm{H}, \mathrm{s}), 7.74(1 \mathrm{H}, \mathrm{t}, J=7.2$ Hz), $7.69(1 \mathrm{H}, \mathrm{d}, J=7.8 \mathrm{~Hz}), 7.60(1 \mathrm{H}, \mathrm{t}, J=7.2 \mathrm{~Hz}), 7.43(2 \mathrm{H}, \mathrm{t}, J=7.2 \mathrm{~Hz}), 7.38(1 \mathrm{H}, \mathrm{t}, J=$ $7.8 \mathrm{~Hz}), 7.23(2 \mathrm{H}, \mathrm{d}, J=8.4 \mathrm{~Hz}), 7.14(2 \mathrm{H}, \mathrm{t}, J=7.8 \mathrm{~Hz}), 7.02(2 \mathrm{H}, \mathrm{d}, J=7.6 \mathrm{~Hz}), 6.94(1 \mathrm{H}$, t, $J=7.2 \mathrm{~Hz}), 4.11(1 \mathrm{H}, \mathrm{d}, J=16.8 \mathrm{~Hz}), 3.99(1 \mathrm{H}, \mathrm{dd}, J=9.0,4.2 \mathrm{~Hz}), 3.87(1 \mathrm{H}, \mathrm{d}, J=16.8$ $\mathrm{Hz}), 3.06(1 \mathrm{H}, \mathrm{dd}, J=18.6,9.0 \mathrm{~Hz}), 2.64(1 \mathrm{H}, \mathrm{d}, J=20.4 \mathrm{~Hz}) ;{ }^{13} \mathrm{C}$ NMR (150 MHz, DMSO$\left.d_{6}\right) \delta 175.4,173.8,170.8,167.4,143.2,151.5,138.0,132.6,131.6,130.2,128.9,128.7,128.6$, $128.5,128.2,127.7,126.6,125.8,123.7,123.5,121.2,119.0,116.2,50.7,49.0,44.8,31.3$. IR (ATR): 1698, 1445, 1404, 1227, $734 \mathrm{~cm}^{-1}$; HRMS $\left(\mathrm{EI}^{+}\right) \mathrm{m} / z\left[\mathrm{M}^{+}\right]$calcd for $\mathrm{C}_{33} \mathrm{H}_{22} \mathrm{Cl}_{2} \mathrm{~N}_{4} \mathrm{O}_{4}$ : 608.1018, Found: 608.1022. 


\section{2-(3-(Tert-butyl)-5-(2,5-dioxo-1-phenylpyrrolidin-3-yl)-6-oxo-5,6-dihydrobenzo[4,5]}

\section{imidazo[2,1-a]isoquinolin-5-yl)- $N$-phenylacetamide (3o):}

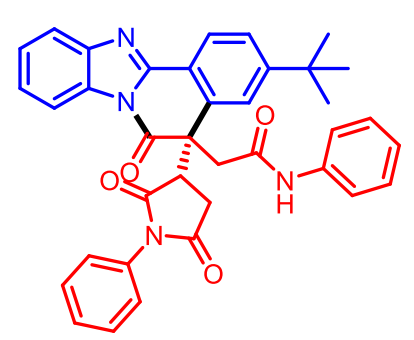

The compound was prepared according to the general procedure and purified by column chromatography (EtOAc/hexane $=25: 75)$.

Yield: $82 \%$ (245 mg); state: off white solid; mp: $230-232{ }^{\circ} \mathrm{C} ;{ }^{1} \mathrm{H}$ NMR (600 MHz, DMSO- $\left.d_{6}\right) \delta 10.30(1 \mathrm{H}, \mathrm{s}), 8.31(2 \mathrm{H}, \mathrm{t}, J=8.4$ $\mathrm{Hz}), 7.86(1 \mathrm{H}, \mathrm{d}, J=8.4 \mathrm{~Hz}), 7.66(1 \mathrm{H}, \mathrm{s}), 7.59(1 \mathrm{H}, \mathrm{dd}, J=8.4$, $1.2 \mathrm{~Hz}), 7.52-7.46(2 \mathrm{H}, \mathrm{m}), 7.40-7.34(3 \mathrm{H}, \mathrm{m}), 7.22(2 \mathrm{H}, \mathrm{d}, J=7.8 \mathrm{~Hz}), 7.13(2 \mathrm{H}, \mathrm{t}, J=7.8$ Hz), $6.96(2 \mathrm{H}, \mathrm{d}, J=7.2 \mathrm{~Hz}), 6.93(1 \mathrm{H}, \mathrm{t}, J=7.2 \mathrm{~Hz}),, 4.22(1 \mathrm{H}, \mathrm{d}, J=16.2 \mathrm{~Hz}), 4.05(1 \mathrm{H}$, dd, $J=9.0,5.4 \mathrm{~Hz},), 3.86(1 \mathrm{H}, \mathrm{d}, J=16.2 \mathrm{~Hz}),, 3.04(1 \mathrm{H}, \mathrm{dd}, J=18.0,9.0 \mathrm{~Hz}),, 2.64(1 \mathrm{H}, \mathrm{d}$, $J=15.6 \mathrm{~Hz},), 1.23(9 \mathrm{H}, \mathrm{s}) ;{ }^{13} \mathrm{C}$ NMR (150 MHz, DMSO- $\left.d_{6}\right) \delta 175.4,173.8,170.9,167.5$, 154.9, 149.6, 143.6, 139.1, 138.1, 131.6, 130.8, 128.8, 128.5, 128.5, 126.5, 125.8, 125.7, 125.4, 125.3, 123.4, 123.1, 121.8, 119.6, 119.2, 115.1, 51.0, 49.2, 44.4, 35.0, 31.2, 30.7. IR (ATR): 1701, 1603, 1410, 1378, $842 \mathrm{~cm}^{-1}$; HRMS $\left(\mathrm{EI}^{+}\right) \mathrm{m} / z\left[\mathrm{M}^{+}\right]$calcd for $\mathrm{C}_{37} \mathrm{H}_{32} \mathrm{~N}_{4} \mathrm{O}_{4}: 596.2424$, Found: 596.2425.

\section{2-(5-(2,5-Dioxo-1-phenylpyrrolidin-3-yl)-6-oxo-3-phenyl-5,6-dihydrobenzo[4,5]imidazo} [2,1-a]isoquinolin-5-yl)- $N$-phenylacetamide (3p):

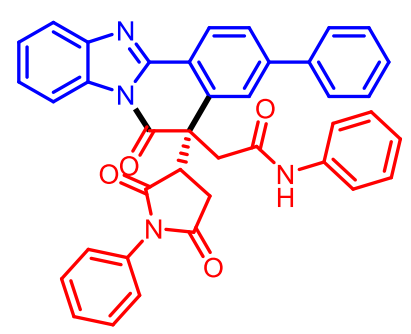

The compound was prepared according to the general procedure and purified by column chromatography $($ EtOAc/hexane $=25: 75)$. Yield: 78\% (241 mg); state: white solid; mp: 200-202 ${ }^{\circ} \mathrm{C} ;{ }^{1} \mathrm{H}$ NMR $\left(600 \mathrm{MHz}, \mathrm{DMSO}-d_{6}\right) \delta 10.31(1 \mathrm{H}, \mathrm{s}), 8.47(1 \mathrm{H}, \mathrm{d}, J=7.8 \mathrm{~Hz})$, $8.31(1 \mathrm{H}, \mathrm{dd}, J=6.6,2.4 \mathrm{~Hz}), 7.97(1 \mathrm{H}, \mathrm{s}), 7.91-7.87(2 \mathrm{H}, \mathrm{m})$, $7.79(2 \mathrm{H}, \mathrm{d}, J=7.8 \mathrm{~Hz}), 7.54-7.45(5 \mathrm{H}, \mathrm{m}), 7.29-7.25(3 \mathrm{H}, \mathrm{m}), 7.21(2 \mathrm{H}, \mathrm{t}, J=6.6 \mathrm{~Hz}), 7.13$ $(2 \mathrm{H}, \mathrm{t}, J=6.6 \mathrm{~Hz}),, 6.92(1 \mathrm{H}, \mathrm{t}, J=7.2 \mathrm{~Hz}), 6.80(2 \mathrm{H}, \mathrm{d}, J=7.2 \mathrm{~Hz}), 4.23(1 \mathrm{H}, \mathrm{d}, J=16.2$ Hz), $4.13(1 \mathrm{H}, \mathrm{dd}, J=9.0,4.8 \mathrm{~Hz}),, 3.90(1 \mathrm{H}, \mathrm{d}, J=16.2 \mathrm{~Hz}$, ), $3.12(1 \mathrm{H}, \mathrm{dd}, J=18.0,9.6 \mathrm{~Hz}$,$) ,$ $2.76(1 \mathrm{H}, \mathrm{d}, J=13.8 \mathrm{~Hz},) ;{ }^{13} \mathrm{C}$ NMR $\left(150 \mathrm{MHz}, \mathrm{DMSO}-d_{6}\right) \delta 175.7,173.9,170.8,167.6,149.4$, $143.6,143.3,138.76,138.2$, 131.5, 130.9, 129.0, 128.7, 128.6, 128.5, 127.0, 126.9, 126.5, 126.1, 125.9, 125.6, 124.6, 123.4, 123.4, 119.8, 119.1, 115.1, 50.9, 49.2, 44.6, 31.5. IR (ATR): 3363, 1707, 1398, 1207, $842 \mathrm{~cm}^{-1}$; HRMS $\left(\mathrm{EI}^{+}\right) \mathrm{m} / z\left[\mathrm{M}^{+}\right]$calcd for $\mathrm{C}_{39} \mathrm{H}_{28} \mathrm{~N}_{4} \mathrm{O}_{4}: 616.2111$, Found: 616.2109. 
2-(5-(2,5-dioxo-1-phenylpyrrolidin-3-yl)-3-(4-fluorophenyl)-6-oxo-5,6-dihydrobenzo[4,5] imidazo[2,1-a]isoquinolin-5-yl)- $N$-phenylacetamide (3q):

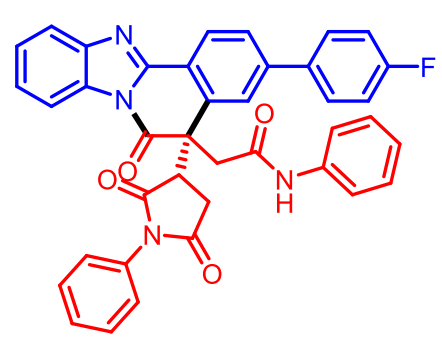

The compound was prepared according to the general procedure and purified by column chromatography (EtOAc/hexane $=$ 25:75). Yield: 76\% (240 mg); State: white solid; mp: 270-272 ${ }^{\circ} \mathrm{C} ;{ }^{1} \mathrm{H}$ NMR (600 MHz, DMSO- $\left.d_{6}\right) \delta 10.30(1 \mathrm{H}, \mathrm{s}), 8.45(1 \mathrm{H}$, $\mathrm{d}, J=6.6 \mathrm{~Hz}), 8.30(1 \mathrm{H}, \mathrm{dd}, J=6.6,1.2 \mathrm{~Hz}), 7.95(1 \mathrm{H}, \mathrm{s}), 7.89-$ $7.87(2 \mathrm{H}, \mathrm{m}), 7.86-7.83(2 \mathrm{H}, \mathrm{m}), 7.52-7.50(2 \mathrm{H}, \mathrm{m}), 7.38(2 \mathrm{H}, \mathrm{t}, J=9 \mathrm{~Hz}), 7.30(1 \mathrm{H}, \mathrm{t}, J=$ $7.2 \mathrm{~Hz}), 7.27-7.22(4 \mathrm{H}, \mathrm{m}), 7.13(2 \mathrm{H}, \mathrm{t}, J=7.8 \mathrm{~Hz}), 6.93(1 \mathrm{H}, \mathrm{t}, J=7.3 \mathrm{~Hz}), 6.80(2 \mathrm{H}, \mathrm{d}, J$ $=7.2 \mathrm{~Hz}), 4.19(1 \mathrm{H}, \mathrm{d}, J=16.2 \mathrm{~Hz}), 4.14(1 \mathrm{H}, \mathrm{dd}, J=9.0,4.2 \mathrm{~Hz}), 3.89(1 \mathrm{H}, \mathrm{d}, J=16.2 \mathrm{~Hz})$, $3.13(1 \mathrm{H}, \mathrm{dd}, J=18.0,9.2 \mathrm{~Hz}), 2.81(1 \mathrm{H}, \mathrm{d}, J=13.8 \mathrm{~Hz}) ;{ }^{13} \mathrm{C}$ NMR (150 MHz, DMSO- $\left.d_{6}\right) \delta$ 175.6, 174.0, 170.8, 167.6, $163.3\left(\mathrm{C}-\mathrm{F},{ }^{1} J \mathrm{C}-\mathrm{F}=244.8 \mathrm{~Hz}\right), 161.7\left(\mathrm{C}-\mathrm{F},{ }^{1} J \mathrm{C}-\mathrm{F}=244.8 \mathrm{~Hz}\right)$, 149.4, 143.6, 142.2, 138.2, 135.2, 131.5, 130.9, $129.2\left(\mathrm{C}-\mathrm{F},{ }^{2} J \mathrm{C}-\mathrm{F}=8.2 \mathrm{~Hz}\right), 129.1\left(\mathrm{C}-\mathrm{F},{ }^{2} J \mathrm{C}-\right.$ $\mathrm{F}=8.2 \mathrm{~Hz}), 128.7,128.6,128.5,126.9,126.5,126.1\left(\mathrm{C}-\mathrm{F},{ }^{3} J \mathrm{C}-\mathrm{F}=24.1 \mathrm{~Hz}\right), 125.9\left(\mathrm{C}-\mathrm{F},{ }^{3} J \mathrm{C}-\right.$ $\mathrm{F}=24.1 \mathrm{~Hz}), 125.6,124.5,123.5,123.3,119.8,119.1,116.0\left(\mathrm{C}-\mathrm{F},{ }^{4} J \mathrm{C}-\mathrm{F}=21.3 \mathrm{~Hz}\right), 115.9$ $\left(\mathrm{C}-\mathrm{F},{ }^{4} \mathrm{JC}-\mathrm{F}=21.3 \mathrm{~Hz}\right), 115.1,50.9,49.3,44.6,31.5 ;{ }^{19} \mathrm{~F}$ NMR $(565 \mathrm{~Hz}$, DMSO-d $) \delta-113.6$ (1F). IR (ATR): 3317, 1692, 1405, 1175, $692 \mathrm{~cm}^{-1}$; HRMS $\left(\mathrm{EI}^{+}\right) \mathrm{m} / z\left[\mathrm{M}^{+}\right]$calcd for $\mathrm{C}_{39} \mathrm{H}_{27} \mathrm{FN}_{4} \mathrm{O}_{4}$ : 634.2016, Found: 634.2018.

\section{2-(3-Chloro-5-(2,5-dioxo-1-phenylpyrrolidin-3-yl)-6-oxo-5,6-dihydrobenzo[4,5]imidazo} $[2,1-a]$ isoquinolin-5-yl)- $N$-phenylacetamide (3r):

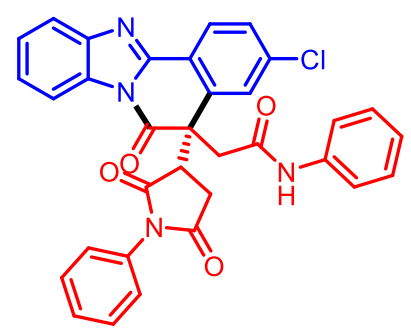

The compound was prepared according to the general procedure and purified by column chromatography (EtOAc/ hexane $=25: 75)$. Yield: 74\% (213 mg); state: white solid; mp: 272-274 ${ }^{\circ} \mathrm{C} ;{ }^{1} \mathrm{H}$ NMR $\left(600 \mathrm{MHz}, \mathrm{DMSO}-d_{6}\right) \delta 10.26(1 \mathrm{H}, \mathrm{s}), 8.38(1 \mathrm{H}, \mathrm{d}, J=8.4 \mathrm{~Hz})$, $8.25(1 \mathrm{H}, \mathrm{dd}, J=6.6,1.2 \mathrm{~Hz}), 7.88-7.85(2 \mathrm{H}, \mathrm{m}), 7.65(1 \mathrm{H}, \mathrm{dd}$, $J=6.6,1.8 \mathrm{~Hz}), 7.51-7.49(2 \mathrm{H}, \mathrm{m}), 7.39-7.34(3 \mathrm{H}, \mathrm{m}), 7.26(2 \mathrm{H}, \mathrm{d}, J=7.8 \mathrm{~Hz}), 7.15(2 \mathrm{H}$, t, $J=7.8 \mathrm{~Hz}), 6.96-6.91(3 \mathrm{H}, \mathrm{m}), 4.06(1 \mathrm{H}, \mathrm{dd}, J=9.0,4.2 \mathrm{~Hz}), 3.99(1 \mathrm{H}, \mathrm{d}, J=16.8 \mathrm{~Hz})$, $3.87(1 \mathrm{H}, \mathrm{d}, J=16.8 \mathrm{~Hz}), 3.12(1 \mathrm{H}, \mathrm{dd}, J=18.0,9.6 \mathrm{~Hz}), 2.93(1 \mathrm{H}, \mathrm{d}, J=17.4 \mathrm{~Hz}) ;{ }^{13} \mathrm{C} \mathrm{NMR}$ (150 MHz, DMSO-d $) \delta 175.4,174.0,170.2$, 167.4, 148.9, 143.3, 140.4, 138.1, 136.5, 131.6, $130.8,130.5,128.9,128.8,128.7,128.6,127.1,126.6,126.3,126.1,125.9,123.6,123.5,119.9$, 
119.1, 115.1, 50.4, 49.3, 44.6, 31.5. IR (ATR): 3350, 2928, 1684, 1539, $1365 \mathrm{~cm}^{-1}$; HRMS $\left(\mathrm{EI}^{+}\right) \mathrm{m} / z\left[\mathrm{M}^{+}\right]$calcd for $\mathrm{C}_{33} \mathrm{H}_{23} \mathrm{ClN}_{4} \mathrm{O}_{4}$ : 574.1408, Found: 574.1406.

\section{2-(3-Bromo-5-(2,5-dioxo-1-phenylpyrrolidin-3-yl)-6-oxo-5,6-dihydrobenzo[4,5]}

imidazo[2,1-a]isoquinolin-5-yl)- $N$-phenylacetamide (3s):

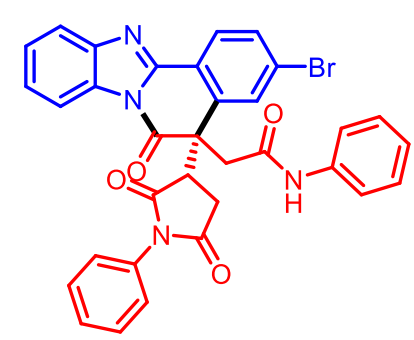

The compound was prepared according to the general procedure and purified by column chromatography (EtOAc/hexane $=25: 75)$. Yield: $73 \%$ (225 mg); state: white solid; mp: $275-277{ }^{\circ} \mathrm{C} ;{ }^{1} \mathrm{H}$ NMR $\left(600 \mathrm{MHz}, \mathrm{DMSO}-d_{6}\right) \delta 10.26(1 \mathrm{H}, \mathrm{s}), 8.29(1 \mathrm{H}, \mathrm{d}, J=8.4 \mathrm{~Hz})$, $8.26-8.23(1 \mathrm{H}, \mathrm{m}), 7.99(1 \mathrm{H}, \mathrm{s}), 7.86(1 \mathrm{H}, \mathrm{dd}, J=7.2,2.4 \mathrm{~Hz})$, $7.78(1 \mathrm{H}, \mathrm{dd}, J=7.8,1.2 \mathrm{~Hz}), 7.52-7.49(2 \mathrm{H}, \mathrm{m}), 7.40-7.34(3 \mathrm{H}, \mathrm{m}), 7.26(2 \mathrm{H}, \mathrm{d}, J=7.8$ $\mathrm{Hz}), 7.15(2 \mathrm{H}, \mathrm{t}, J=7.8 \mathrm{~Hz}), 6.95(3 \mathrm{H}, \mathrm{t}, J=7.2 \mathrm{~Hz}), 4.05(1 \mathrm{H}, \mathrm{dd}, J=9.0,4.2 \mathrm{~Hz}), 4.00(1 \mathrm{H}$, d, $J=16.7 \mathrm{~Hz}), 3.86(1 \mathrm{H}, \mathrm{d}, J=16.8 \mathrm{~Hz}), 3.11(1 \mathrm{H}, \mathrm{dd}, J=18.0,9.0 \mathrm{~Hz}), 2.90(1 \mathrm{H}, \mathrm{d}, J=16.8$ $\mathrm{Hz}) ;{ }^{13} \mathrm{C}$ NMR (150 MHz, DMSO- $\left.d_{6}\right) \delta 175.4,173.9,170.1,167.4$, 149.0, 143.3, 140.4, 138.1, $131.7,131.5,130.8,129.1,128.9,128.6,128.6,127.2$, 126.6, 126.0, 125.8, 125.4, 123.8, 123.5, 119.9, 119.14, 115.0, 50.4, 49.2, 44.6, 31.5. IR (ATR): 3360, 1710, 1600, 1552, $992 \mathrm{~cm}^{-1}$; HRMS $\left(\mathrm{EI}^{+}\right) \mathrm{m} / z\left[\mathrm{M}^{+}\right]$calcd for $\mathrm{C}_{33} \mathrm{H}_{23} \mathrm{BrN}_{4} \mathrm{O}_{4}$ : 618.0903, Found: 618.0905.

\section{2-(5-(2,5-Dioxo-1-phenylpyrrolidin-3-yl)-6-oxo-3-(trifluoromethyl)-5,6-}

dihydrobenzo[4,5]imidazo[2,1- $a]$ isoquinolin-5-yl)- $N$-phenylacetamide $(3 t)$

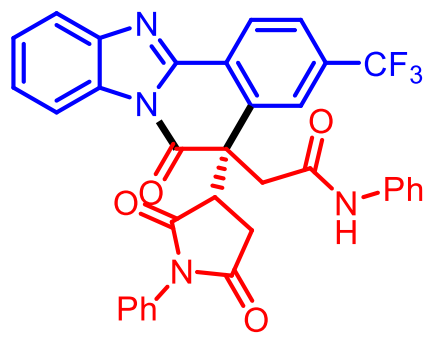

The compound was prepared according to the general procedure and purified by column chromatography $($ EtOAc/hexane $=25: 75)$. Yield: $78 \%$ (237 mg); state: white solid; mp: $240-242{ }^{\circ} \mathrm{C} ;{ }^{1} \mathrm{H}$ NMR (600 MHz, DMSO- $\left.d_{6}\right) \delta 10.267(1 \mathrm{H}, \mathrm{s}), 8.57(1 \mathrm{H}, \mathrm{d}, J=8.4$ $\mathrm{Hz}), 8.29-8.26(1 \mathrm{H}, \mathrm{m}), 8.16(\mathrm{~s}, 1 \mathrm{H}), 7.93-7.90(2 \mathrm{H}, \mathrm{m}), 7.55-$ $7.52(2 \mathrm{H}, \mathrm{m}), 7.34-7.31(3 \mathrm{H}, \mathrm{m}), 7.21(2 \mathrm{H}, \mathrm{d}, J=7.2 \mathrm{~Hz}), 7.13(1 \mathrm{H}, \mathrm{t}, J=7.2 \mathrm{~Hz}), 6.94(1 \mathrm{H}$, t, $J=7.2 \mathrm{~Hz}), 6.88-6.85(2 \mathrm{H}, \mathrm{m}), 4.18-4.15(1 \mathrm{H}, \mathrm{m}), 4.03(1 \mathrm{H}, \mathrm{d}, J=16.6 \mathrm{~Hz}), 3.90(1 \mathrm{H}$, $\mathrm{d}, J=16.6 \mathrm{~Hz}), 3.17-3.10(2 \mathrm{H}, \mathrm{m}) .{ }^{13} \mathrm{C} \mathrm{NMR}\left(150 \mathrm{MHz}, \mathrm{DMSO}-d_{6}\right) \delta 175.4,174.0,170.2$, $167.4,148.5,143.3,139.9,138.0,131.5,131.3,130.8,128.8,128.6,128.6,128.1,126.5,126.3$, 126.3, $126.3\left(\mathrm{C}-\mathrm{F},{ }^{2} J \mathrm{C}-\mathrm{F}=11.7 \mathrm{~Hz}\right), 126.2\left(\mathrm{C}-\mathrm{F},{ }^{2} J \mathrm{C}-\mathrm{F}=11.7 \mathrm{~Hz}\right), 125.2,124.6\left(\mathrm{C}-\mathrm{F},{ }^{1} \mathrm{JC}-\mathrm{F}\right.$ $=271.3 \mathrm{~Hz}), 123.6,123.4,122.8\left(\mathrm{C}-\mathrm{F},{ }^{1} \mathrm{JC}-\mathrm{F}=271.3 \mathrm{~Hz}\right), 120.2,119.2,115.1,50.3,49.4$, 44.8, 
31.6; ${ }^{19} \mathrm{~F}$ NMR (565 Hz, DMSO- $\left.d_{6}\right) \delta-61.1$ (1F). IR (ATR): 3345, 1737, 1602, 1497, $1243 \mathrm{~cm}^{-}$ 1; HRMS (ESI) $m / z\left[\mathrm{M}+\mathrm{H}^{+}\right]$calcd for $\mathrm{C}_{34} \mathrm{H}_{24} \mathrm{~F}_{3} \mathrm{~N}_{4} \mathrm{O}_{4}$ : 609.1744, Found: 609.1740 .

\section{2-(5-(2,5-Dioxo-1-phenylpyrrolidin-3-yl)-6-oxo-5,6-dihydronaphtho[2',3' :4,5]imidazo}

\section{[2,1-a]isoquinolin-5-yl)- $N$-phenylacetamide (3u):}

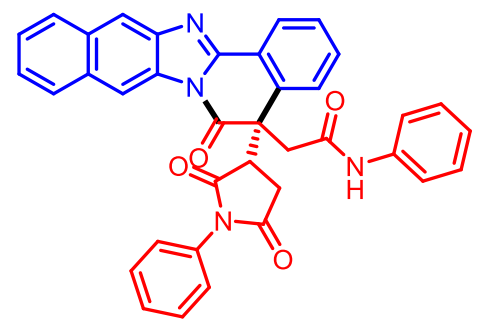

The compound was prepared according to the general procedure and purified by column chromatography (EtOAc/ hexane $=25: 75)$. Yield: $73 \%$ (215 mg); state: white solid; mp: 203-205 ${ }^{\circ} \mathrm{C} ;{ }^{1} \mathrm{H}$ NMR (600 MHz, DMSO-d 6$) \delta 10.26$ (1H,s), $8.81(1 \mathrm{H}, \mathrm{s}), 8.49(1 \mathrm{H}, \mathrm{d}, \mathrm{J}=8.4 \mathrm{~Hz}), 8.41(1 \mathrm{H}, \mathrm{s}), 8.17-8.14$ $(2 \mathrm{H}, \mathrm{m}), 7.76-7.71(2 \mathrm{H}, \mathrm{m}), 7.61(1 \mathrm{H}, \mathrm{t}, \mathrm{J}=7.2 \mathrm{~Hz}), 7.59-$ $7.54(2 \mathrm{H}, \mathrm{m}), 7.40-7.32(3 \mathrm{H}, \mathrm{m}), 7.24(2 \mathrm{H}, \mathrm{d}, \mathrm{J}=7.8 \mathrm{~Hz}), 7.12(2 \mathrm{H}, \mathrm{t}, \mathrm{J}=7.2 \mathrm{~Hz}), 6.98(2 \mathrm{H}$, d, J = 7.2 Hz), $6.92(1 \mathrm{H}, \mathrm{t}, \mathrm{J}=7.2 \mathrm{~Hz}), 4.08(1 \mathrm{H}, \mathrm{d}, \mathrm{J}=15.6 \mathrm{~Hz}), 4.04-4.00(1 \mathrm{H}, \mathrm{m}), 3.90$ $(1 \mathrm{H}, \mathrm{d}, \mathrm{J}=16.8 \mathrm{~Hz}), 3.09(1 \mathrm{H}, \mathrm{dd}, \mathrm{J}=18.0,9.6 \mathrm{~Hz}), 2.75(1 \mathrm{H}, \mathrm{d}, \mathrm{J}=19.8 \mathrm{~Hz}) ;{ }^{13} \mathrm{C} \mathrm{NMR}(150$ MHz, DMSO-d 6 ) $\delta 175.6,174.0,170.2,167.5,152.2,142.8,138.2,132.6,131.7,131.6,131.4$, 130.6, 128.9, 128.6, 128.4, 128.3, 126.6, 126.2, 126.0, 125.6, 125.4, 124.2, 123.4, 119.0, 117.0, 112.4, 50.6, 49.2, 44.5, 31.4. IR (ATR): 3341, 1704, 1600, 1374, $\left.745 \mathrm{~cm}^{-1} ; \mathrm{HRMS}_{(\mathrm{EI}}{ }^{+}\right) \mathrm{m} / \mathrm{z}$ $\left[\mathrm{M}^{+}\right]$calcd for $\mathrm{C}_{37} \mathrm{H}_{26} \mathrm{~N}_{4} \mathrm{O}_{4}: 590.1954$, Found: 590.1957 .

\section{2-(5-(2,5-Dioxo-1-phenylpyrrolidin-3-yl)-3-(naphthalen-2-yl)-6-oxo-5,6-dihydrobenzo} $[4,5]$ imidazo[2,1-a]isoquinolin-5-yl)- $N$-phenylacetamide $(3 \mathrm{v})$ :

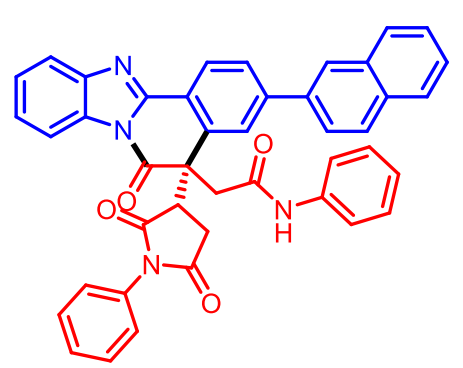

The compound was prepared according to the general procedure and purified by column chromatography $($ EtOAc/hexane $=25: 75)$. Yield: $81 \%(270 \mathrm{mg})$; state: white solid; mp: $189-191{ }^{\circ} \mathrm{C} ;{ }^{1} \mathrm{H}$ NMR (600 MHz, DMSO-d 6 ) $\delta 10.34$ $(1 \mathrm{H}, \mathrm{s}), 8.50(1 \mathrm{H}, \mathrm{d}, J=8.4 \mathrm{~Hz}), 8.31(2 \mathrm{H}, \mathrm{d}, J=7.2 \mathrm{~Hz}), 8.10$ $(1 \mathrm{H}, \mathrm{s}), 8.07(1 \mathrm{H}, \mathrm{d}, J=8.4 \mathrm{~Hz}), 8.04(1 \mathrm{H}, \mathrm{dd}, J=9.0,1.2 \mathrm{~Hz})$, $8.00(1 \mathrm{H}, \mathrm{d}, J=7.2 \mathrm{~Hz}), 7.94(2 \mathrm{H}, \mathrm{d}, \mathrm{J}=7.2 \mathrm{~Hz}), 7.90-7.88(1 \mathrm{H}, \mathrm{m}), 7.59(2 \mathrm{H}, \mathrm{m}), 7.52(2 \mathrm{H}$, m), $7.27(2 \mathrm{H}, \mathrm{d}, J=7.8 \mathrm{~Hz}), 7.23(1 \mathrm{H}, \mathrm{t}, J=7.5 \mathrm{~Hz}), 7.12(4 \mathrm{H}, \mathrm{t}, J=7.8 \mathrm{~Hz}), 6.91(1 \mathrm{H}, \mathrm{t}, J=$ $7.8 \mathrm{~Hz}), 6.80(2 \mathrm{H}, \mathrm{d}, J=7.2 \mathrm{~Hz}), 4.26(1 \mathrm{H}, \mathrm{d}, J=16.2 \mathrm{~Hz}), 4.15(1 \mathrm{H}, \mathrm{dd}, J=9.0,4.8 \mathrm{~Hz}), 3.92$ $(1 \mathrm{H}, \mathrm{d}, J=16.2 \mathrm{~Hz}), 3.14(1 \mathrm{H}, \mathrm{dd}, J=18.0,9.0 \mathrm{~Hz}), 2.79(1 \mathrm{H}, \mathrm{d}, J=10.2 \mathrm{~Hz}) ;{ }^{13} \mathrm{C} \mathrm{NMR}(150$ MHz, DMSO-d 6 ) $\delta 175.7,174.0,170.8,167.6,149.4,143.6,143.2,138.2,136.1,133.1,132.6$, $131.5,130.9,128.7,128.6,128.4,128.2$, 127.6, 127.2 , 126.7, 126.5, 126.0, 125.9, 125.6, 124.9, 
123.4, 123.4, 119.8, 119.1, 115.1, 50.9, 49.3, 44.6, 31.5. IR: 3250, 1710, 1599, 1360, $750 \mathrm{~cm}^{-}$ 1; HRMS: $m / z\left[\mathrm{M}+\mathrm{H}^{+}\right]$calcd for $\mathrm{C}_{43} \mathrm{H}_{31} \mathrm{~N}_{4} \mathrm{O}_{4}$ : 667.2339, Found: 667.2343.

\section{2-(5-(2,5-Dioxo-1-phenylpyrrolidin-3-yl)-10-methyl-6-oxo-5,6-}

dihydrobenzo $[4,5]$ imidazo $[2,1-a]$ isoquinolin-5-yl)- $N$-phenylacetamide (3w) and 2-(5-(2,5Dioxo-1-phenylpyrrolidin-3-yl)-9-methyl-6-oxo-5,6-dihydrobenzo[4,5]imidazo[2,1a]isoquinolin-5-yl)- $N$-phenylacetamide $\left(3 w^{\prime}\right)$ :

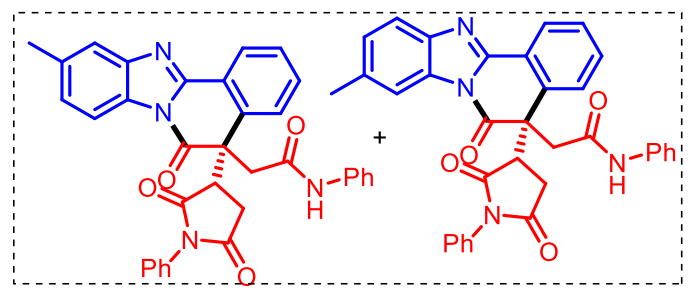

The compound was prepared according to the general procedure and purified by column chromatography (EtOAc/hexane $=25: 75)$. Combined yield: $75 \%$ (208 mg); state: white solid; ${ }^{1} \mathrm{H}$ NMR $\left(600 \mathrm{MHz}, \mathrm{DMSO}-d_{6}\right) \delta 10.29(2 \mathrm{H}, \mathrm{d}, J=$ $3.6 \mathrm{~Hz}), 8.43-8.41(2 \mathrm{H}, \mathrm{m}), 8.17-8.15(2 \mathrm{H}, \mathrm{m}), 7.77(1 \mathrm{H}, \mathrm{d}, J=8.4 \mathrm{~Hz}), 7.70-7.68(5 \mathrm{H}$, m), $7.60-7.56(2 \mathrm{H}, \mathrm{m}), 7.45-7.42(4 \mathrm{H}, \mathrm{m}), 7.40-7.37(2 \mathrm{H}, \mathrm{m}), 7.35-7.32(2 \mathrm{H}, \mathrm{m}), 7.29$ $(4 \mathrm{H}, \mathrm{d}, J=7.8 \mathrm{~Hz}), 7.15(4 \mathrm{H}, \mathrm{t}, J=7.8 \mathrm{~Hz}), 7.03-7.00(4 \mathrm{H}, \mathrm{m}), 6.95(2 \mathrm{H}, \mathrm{t}, J=7.2 \mathrm{~Hz}), 4.16$ - $4.11(2 \mathrm{H}, \mathrm{m}), 3.98(2 \mathrm{H}, \mathrm{dd}, J=9.4,4.2 \mathrm{~Hz}), 3.90(2 \mathrm{H}, \mathrm{d}, J=16.8 \mathrm{~Hz}), 3.10-3.05(2 \mathrm{H}, \mathrm{m})$, $2.62-2.56(2 \mathrm{H}, \mathrm{m}), 2.51(6 \mathrm{H}, \mathrm{s}) .{ }^{13} \mathrm{C}$ NMR $\left(150 \mathrm{MHz}, \mathrm{DMSO}-d_{6}\right) \delta 175.5,175.5,173.9,170.8$, 170.6, 167.5, 167.5, 149.6, 149.0, 143.8, 141.5, 138.2 137.3, 137.0, 135.5, 135.4, 131.8, 131.7, 131.6, 131.1, 129.0, 128.9, 128.6, 128.6, 128.5, 127.0, 126.7, 126.6, 126.6, 126.1, 125.5, 125.4, 124.6, 124.6, 123.4, 119.7, 119.3, 119.0, 119.0, 115.2, 114.6, 50.8, 50.7, 49.1, 44.4, 44.4, 31.3, 21.4, 21.2; IR (ATR): 3391, 1738, 1646, 1375, $1024 \mathrm{~cm}^{-1}$; HRMS (ESI) $\mathrm{m} / z$ [M+H'] calcd for $\mathrm{C}_{34} \mathrm{H}_{27} \mathrm{~N}_{4} \mathrm{O}_{4}$ : 555.2027, Found: 555.2029.

\section{2-(10-Chloro-5-(2,5-dioxo-1-phenylpyrrolidin-3-yl)-6-oxo-5,6-} dihydrobenzo[4,5]imidazo[2,1-a]isoquinolin-5-yl)- $N$-phenylacetamide $(3 x)$ and 2-(9Chloro-5-(2,5-dioxo-1-phenylpyrrolidin-3-yl)-6-oxo-5,6-dihydrobenzo[4,5]imidazo[2,1a]isoquinolin-5-yl)- $N$-phenylacetamide $\left(3 \mathbf{x}^{\prime}\right)$ :

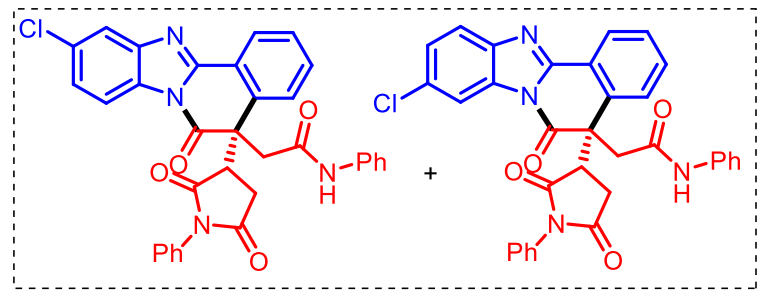

The compound was prepared according to the general procedure and purified by column chromatography $($ EtOAc/hexane $=25: 75)$. Combined yield: $72 \%$ (208 mg); state: white solid; ${ }^{1} \mathrm{H}$ NMR $\left(600 \mathrm{MHz}, \mathrm{CDCl}_{3}\right) \delta 8.55-$ $8.53(2 \mathrm{H}, \mathrm{m}), 8.39(1 \mathrm{H}, \mathrm{d}, J=1.8 \mathrm{~Hz}), 8.25(1 \mathrm{H}, \mathrm{d}, J=8.4 \mathrm{~Hz}), 7.77(1 \mathrm{H}, \mathrm{d}, J=1.2 \mathrm{~Hz}), 7.71$ $(1 \mathrm{H}, \mathrm{d}, J=8.4 \mathrm{~Hz}), 7.60(2 \mathrm{H}, \mathrm{s}), 7.55-7.52(2 \mathrm{H}, \mathrm{m}), 7.46-7.35(12 \mathrm{H}, \mathrm{m}), 7.13-7.08(8 \mathrm{H}$, 
m), $7.00(4 \mathrm{H}, \mathrm{d}, J=7.8 \mathrm{~Hz}), 6.96(2 \mathrm{H}, \mathrm{t}, J=6.0 \mathrm{~Hz}), 4.25(1 \mathrm{H}, \mathrm{d}, J=3.6 \mathrm{~Hz}), 4.22(1 \mathrm{H}, \mathrm{d}, J=$ $3.6 \mathrm{~Hz}), 3.96(1 \mathrm{H}, \mathrm{d}, J=4.8 \mathrm{~Hz}), 3.93(1 \mathrm{H}, \mathrm{d}, J=4.8 \mathrm{~Hz}), 3.75-3.73(2 \mathrm{H}, \mathrm{m}), 2.70-2.64$ $(2 \mathrm{H}, \mathrm{m}), 2.17(1 \mathrm{H}, \mathrm{d}, J=4.2 \mathrm{~Hz}), 2.14(1 \mathrm{H}, \mathrm{d}, J=4.2 \mathrm{~Hz}) .{ }^{13} \mathrm{C} \mathrm{NMR}\left(150 \mathrm{MHz}, \mathrm{CDCl}_{3}\right) \delta$ $175.7,172.8,172.8,170.4,170.3,166.8,166.8,150.2$, 149.6, 144.9, 142.5, 136.9, 134.8, 132.0, 131.9, 131.7, 131.7, 131.5, 131.1, 129.9, 129.4, 129.4, 129.2, 129.1, 128.9, 128.8, 127.2, 127.1, 126.7, 126.3, 126.1 , 125.4, 125.1, 125.1, 124.5, 120.7, 120.0, 119.6, 119.6, 116.3, 115.9, 52.0, 52.0, 48.4, 44.3, 30.5; IR (ATR): 1706, 1598, 1355, 1168, $923 \mathrm{~cm}^{-1}$; HRMS (ESI) $\mathrm{m} / z$ [M+H $\left.{ }^{+}\right]$ calcd for $\mathrm{C}_{33} \mathrm{H}_{24} \mathrm{ClN}_{4} \mathrm{O}_{4}$ : 575.1481, Found: 575.1478.

\section{2-Phenyl-3a,13a-dihydro-1 $H$-benzo[4,5]imidazo[2,1-a]pyrrolo $[3,4-c]$ isoquinoline-}

\section{1,3(2H)-dione (4a):}

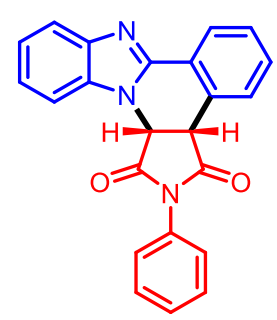

The compound was prepared according to the general procedure and purified by column chromatography $($ EtOAc/hexane $=20: 80)$. Yield: $74 \%$ (135 mg); state: orange solid; mp: 204-206 ${ }^{\circ} \mathrm{C}$; ${ }^{1} \mathrm{H}$ NMR (600 MHz, $\left.\mathrm{CDCl}_{3}\right) \delta 8.41(1 \mathrm{H}, \mathrm{d}, J=7.8 \mathrm{~Hz}), 7.91(1 \mathrm{H}, \mathrm{d}, J=7.8 \mathrm{~Hz}), 7.75(1 \mathrm{H}, \mathrm{d}, J$

$=7.2 \mathrm{~Hz}), 7.70(1 \mathrm{H}, \mathrm{d}, J=7.8 \mathrm{~Hz}), 7.47(2 \mathrm{H}, \mathrm{t}, J=8.4 \mathrm{~Hz}), 7.42(2 \mathrm{H}, \mathrm{t}, J$ $=7.8 \mathrm{~Hz}), 7.38(1 \mathrm{H}, \mathrm{d}, J=7.2 \mathrm{~Hz}), 7.29(2 \mathrm{H}, \mathrm{t}, J=7.8 \mathrm{~Hz}), 7.25(2 \mathrm{H}, \mathrm{d}, J=8.4 \mathrm{~Hz}), 5.84(1 \mathrm{H}$, d, $J=9.6 \mathrm{~Hz}), 4.82(1 \mathrm{H}, \mathrm{d}, J=9.6 \mathrm{~Hz}) ;{ }^{13} \mathrm{C} \mathrm{NMR}\left(150 \mathrm{MHz}, \mathrm{CDCl}_{3}\right) \delta 172.8,171.2,145.1$, $134.5,131.3,130.8,129.3,129.2,129.1,128.1,126.1,126.1,126.0,124.0,123.8,119.2,111.3$, 53.3, 41.7. IR: 2931, 1696, 1555, 1026, $766 \mathrm{~cm}^{-1}$; HRMS $\left(\mathrm{EI}^{+}\right) \mathrm{m} / z\left[\mathrm{M}^{+}\right]$calcd for $\mathrm{C}_{23} \mathrm{H}_{15} \mathrm{~N}_{3} \mathrm{O}_{2}$ : 365.1164, Found: 365.1166.

\section{2-(4-Methoxyphenyl)-3a,13a-dihydro-1H-benzo[4,5]imidazo[2,1-a]pyrrolo[3,4-} c]isoquinoline-1,3(2H)-dione (4b):

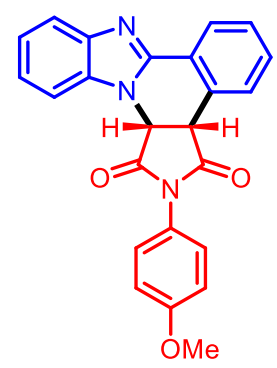

The compound was prepared according to the general procedure and purified by column chromatography $($ EtOAc/hexane $=20: 80)$. Yield: $77 \%$ (153 mg); state: white solid; mp: 242-244 ${ }^{\circ} \mathrm{C}$; ${ }^{1} \mathrm{H}$ NMR (600 MHz, DMSO$\left.d_{6}\right) \delta 8.31(1 \mathrm{H}, \mathrm{d}, J=7.2 \mathrm{~Hz}), 7.87(1 \mathrm{H}, \mathrm{d}, J=7.2 \mathrm{~Hz}), 7.81(1 \mathrm{H}, \mathrm{d}, J=8.4$ $\mathrm{Hz}), 7.72(1 \mathrm{H}, \mathrm{d}, J=8.4 \mathrm{~Hz}), 7.60-7.53(2 \mathrm{H}, \mathrm{m}), 7.33(1 \mathrm{H}, \mathrm{t}, J=7.2 \mathrm{~Hz})$, $7.28(1 \mathrm{H}, \mathrm{t}, J=7.1 \mathrm{~Hz}), 7.22(2 \mathrm{H}, \mathrm{d}, J=8.4 \mathrm{~Hz}), 7.01(2 \mathrm{H}, \mathrm{d}, J=9.0 \mathrm{~Hz})$, $6.28(1 \mathrm{H}, \mathrm{d}, J=9.6 \mathrm{~Hz}), 5.06(1 \mathrm{H}, \mathrm{d}, J=10.2 \mathrm{~Hz}), 3.77(3 \mathrm{H}, \mathrm{s}) ;{ }^{13} \mathrm{C}$ NMR $(150 \mathrm{MHz}, \mathrm{DMSO}-$ $\left.d_{6}\right) \delta 174.1,172.8,159.2,145.6,143.4,135.1,130.6,128.6,128.1,128.0,125.1,124.4,124.1$, 
122.9, 122.7, 119.0, 114.2, 111.8, 55.3, 53.1, 41.5. IR (ATR): 1736, 1372, 1235, 1044, $730 \mathrm{~cm}^{-}$ 1; HRMS $\left(\mathrm{EI}^{+}\right) \mathrm{m} / z\left[\mathrm{M}+\mathrm{H}^{+}\right]$calcd for $\mathrm{C}_{24} \mathrm{H}_{18} \mathrm{~N}_{3} \mathrm{O}_{3}: 396.1342$, Found: 396.1339.

\section{2-(4-Bromophenyl)-3a,13a-dihydro-1H-benzo[4,5]imidazo[2,1-a]pyrrolo[3,4-} $c$ ]isoquinoline-1,3(2H)-dione (4c):

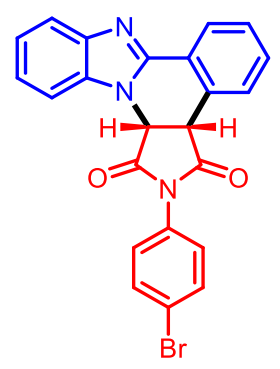

The compound was prepared according to the general procedure and purified by column chromatography (EtOAc/hexane $=20: 80)$. Yield: $72 \%$ (160 mg); state: brown solid; mp: 220-222 ${ }^{\circ} \mathrm{C}$; ${ }^{1} \mathrm{H}$ NMR $(600 \mathrm{MHz}$, DMSO- $\left.d_{6}\right) \delta 8.31(1 \mathrm{H}, \mathrm{d}, J=7.2 \mathrm{~Hz}), 7.87(1 \mathrm{H}, \mathrm{d}, J=7.8 \mathrm{~Hz}), 7.82(1 \mathrm{H}$, $\mathrm{d}, J=7.8 \mathrm{~Hz}), 7.73(1 \mathrm{H}, \mathrm{d}, J=7.8 \mathrm{~Hz}), 7.70(2 \mathrm{H}, \mathrm{d}, J=9 \mathrm{~Hz}), 7.59(1 \mathrm{H}$, $\mathrm{t}, J=7.2 \mathrm{~Hz}), 7.55(1 \mathrm{H}, \mathrm{t}, J=7.2 \mathrm{~Hz}), 7.35-7.27(4 \mathrm{H}, \mathrm{m}), 6.28(1 \mathrm{H}, \mathrm{d}$, $J=9.6 \mathrm{~Hz}), 5.08(1 \mathrm{H}, \mathrm{d}, J=10.2 \mathrm{~Hz}) ;{ }^{13} \mathrm{C} \mathrm{NMR}\left(150 \mathrm{MHz}, \mathrm{DMSO}-d_{6}\right) \delta 173.6,172.2,145.6$, 135.0, 131.9, 131.0, 130.6, 129.0, 128.5, 128.1, 127.8, 125.0, 122.9, 122.7, 121.7, 118.9, 111.9, 53.1, 41.4. IR (ATR): 3342, 2925, 1716, 1366, $692 \mathrm{~cm}^{-1} ; \mathrm{HRMS}\left(\mathrm{EI}^{+}\right) \mathrm{m} / z\left[\mathrm{M}+\mathrm{H}^{+}\right]$calcd for $\mathrm{C}_{23} \mathrm{H}_{14} \mathrm{BrN}_{3} \mathrm{O}_{2}$ : 443.0269, Found: 443.0267.

\section{2-Methyl-3a,13a-dihydro-1H-benzo[4,5]imidazo[2,1-a]pyrrolo[3,4-c]isoquinoline-}

\section{1,3(2H)-dione (4d):}

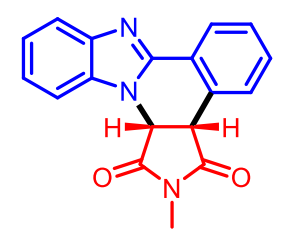

The compound was prepared according to the general procedure and purified by column chromatography $($ EtOAc/hexane $=20: 80)$. Yield: $78 \%(118 \mathrm{mg})$; state: off white solid; mp: $210-212{ }^{\circ} \mathrm{C} ;{ }^{1} \mathrm{H}$ NMR $\left(600 \mathrm{MHz}, \mathrm{CDCl}_{3}\right) \delta 8.38$ $(1 \mathrm{H}, \mathrm{d}, J=7.8 \mathrm{~Hz}), 7.87(1 \mathrm{H}, \mathrm{d}, J=7.2 \mathrm{~Hz}), 7.76(1 \mathrm{H}, \mathrm{d}, J=7.8 \mathrm{~Hz}), 7.70$ $(1 \mathrm{H}, \mathrm{d}, J=7.8 \mathrm{~Hz}), 7.48-7.434(2 \mathrm{H}, \mathrm{m}), 7.32-7.28(2 \mathrm{H}, \mathrm{m}), 5.59(1 \mathrm{H}, \mathrm{d}, J=10.2 \mathrm{~Hz}), 4.64$ $(1 \mathrm{H}, \mathrm{d}, J=10.2 \mathrm{~Hz}), 3.02(3 \mathrm{H}, \mathrm{s}) ;{ }^{13} \mathrm{C} \mathrm{NMR}\left(150 \mathrm{MHz}, \mathrm{CDCl}_{3}\right) \delta 173.9,172.2,145.3,134.7$, 130.9, 129.2, 128.2 126.2, 125.9, 123.8, 123.6, 119.6, 111.2, 53.1, 41.5, 25.6. IR (ATR): 3406, 1023, 998, 824, $762 \mathrm{~cm}^{-1}$; HRMS $\left(\mathrm{EI}^{+}\right) \mathrm{m} / z\left[\mathrm{M}^{+}\right]$calcd for $\mathrm{C}_{18} \mathrm{H}_{13} \mathrm{~N}_{3} \mathrm{O}_{2}$ : 303.1008, Found: 303.1006 . 
2-Ethyl-3a,13a-dihydro-1H-benzo[4,5]imidazo[2,1-a]pyrrolo[3,4-c]isoquinoline $-1,3(2 H)$ dione (4e):

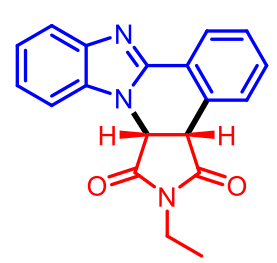

The compound was prepared according to the general procedure and purified by column chromatography (EtOAc/hexane $=20: 80)$. Yield: $75 \%(119 \mathrm{mg})$; state: off white solid; mp: $216-218{ }^{\circ} \mathrm{C} ;{ }^{1} \mathrm{H}$ NMR (600 MHz, DMSO- $d_{6}$ ) $\delta$ $8.26(1 \mathrm{H}, \mathrm{dd}, J=7.8,1.8 \mathrm{~Hz}), 7.85(1 \mathrm{H}, \mathrm{d}, J=7.8 \mathrm{~Hz}), 7.81(1 \mathrm{H}, \mathrm{d}, J=7.8$ $\mathrm{Hz}), 7.71(1 \mathrm{H}, \mathrm{d}, J=7.8 \mathrm{~Hz}), 7.57(1 \mathrm{H}, \mathrm{td}, J=7.2,1.2 \mathrm{~Hz}), 7.54-7.50(1 \mathrm{H}, \mathrm{m}), 7.35-7.31$ $(1 \mathrm{H}, \mathrm{m}), 7.29-7.26(1 \mathrm{H}, \mathrm{m}), 6.13(1 \mathrm{H}, \mathrm{d}, J=9.6 \mathrm{~Hz}), 4.90(1 \mathrm{H}, \mathrm{d}, J=9.6 \mathrm{~Hz}), 3.45(2 \mathrm{H}, \mathrm{q}, J$ $=7.2 \mathrm{~Hz}), 1.04(3 \mathrm{H}, \mathrm{t}, J=7.2 \mathrm{~Hz}) ;{ }^{13} \mathrm{C} \mathrm{NMR}\left(150 \mathrm{MHz}, \mathrm{CDCl}_{3}\right) \delta 174.5,173.2,145.4,143.3$, 135.0, 130.6, 128.6, 128.1, 128.0, 125.0, 124.1, 122.9, 122.7, 119.0, 111.9, 52.8, 41.4, 33.9, 12.3. IR (ATR): $3406,1124,1004,712,762 \mathrm{~cm}^{-1}$; $\mathrm{HRMS}\left(\mathrm{EI}^{+}\right) \mathrm{m} / z\left[\mathrm{M}^{+}\right]$calcd for $\mathrm{C}_{19} \mathrm{H}_{15} \mathrm{~N}_{3} \mathrm{O}_{2}$ : 317.1164, Found: 317.1162.

\section{2-Propyl-3a,13a-dihydro-1H-benzo[4,5]imidazo[2,1-a]pyrrolo[3,4-c]isoquinoline- 1,3(2H)-dione (4f):}

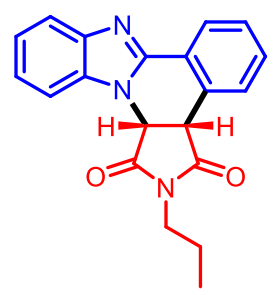

The compound was prepared according to the general procedure and purified by column chromatography (EtOAc/hexane $=20: 80)$. Yield: 74\% (123 mg); state: orange solid; mp: $225-227{ }^{\circ} \mathrm{C} ;{ }^{1} \mathrm{H} \mathrm{NMR}\left(600 \mathrm{MHz}, \mathrm{CDCl}_{3}\right) \delta 8.38(1 \mathrm{H}$, $\mathrm{d}, J=7.8 \mathrm{~Hz}), 7.87(1 \mathrm{H}, \mathrm{d}, J=7.8 \mathrm{~Hz}), 7.72(1 \mathrm{H}, \mathrm{d}, J=8.4), 7.72(1 \mathrm{H}, \mathrm{d}, J=$ 7.8), $7.48-7.41(2 \mathrm{H}, \mathrm{m}), 7.33-7.26(2 \mathrm{H}, \mathrm{m}), 5.65(1 \mathrm{H}, \mathrm{d}, J=9.6 \mathrm{~Hz}), 4.67$ $(1 \mathrm{H} \mathrm{d}, J=9.6 \mathrm{~Hz}), 3.52-3.44(2 \mathrm{H}, \mathrm{m}), 1.55(2 \mathrm{H}, \mathrm{q}, J=7.2 \mathrm{~Hz}), 0.79(3 \mathrm{H}, \mathrm{t}, J=7.8 \mathrm{~Hz}) ;{ }^{13} \mathrm{C}$ NMR (150 MHz, $\left.\mathrm{CDCl}_{3}\right) \delta 173.9,172.2,145.1,134.5,131.2,129.2,128.1,126.4,126.0,123.9$, 123.8, 119.2, 111.3, 53.1, 41.5, 41.3, 20.7, 11.0. IR (ATR): 3305, 1158, 998, 815, $703 \mathrm{~cm}^{-1}$; HRMS $\left(\mathrm{EI}^{+}\right) \mathrm{m} / z\left[\mathrm{M}^{+}\right]$calcd for $\mathrm{C}_{20} \mathrm{H}_{17} \mathrm{~N}_{3} \mathrm{O}_{2}: 331.1321$, Found: 331.1319 .

\section{2-Undecyl-3a,13a-dihydro-1H-benzo[4,5]imidazo[2,1-a]pyrrolo[3,4-c] isoquinoline-}

\section{1,3(2H)-dione (4g):}

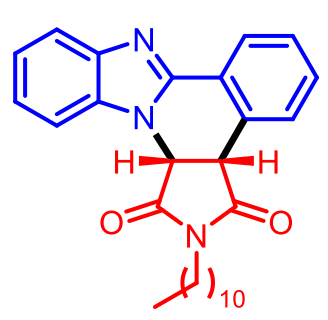

The compound was prepared according to the general procedure and purified by column chromatography $($ EtOAc/hexane $=15: 85)$. Yield: $70 \%$ (155 mg); state: viscous liquid. ${ }^{1} \mathrm{H} \mathrm{NMR}\left(600 \mathrm{MHz}, \mathrm{CDCl}_{3}\right) \delta$ 8.38 - $8.37(1 \mathrm{H}, \mathrm{m}), 7.87-7.85(1 \mathrm{H}, \mathrm{m}), 7.78-7.76(1 \mathrm{H}, \mathrm{m}), 7.69-$ $7.68(1 \mathrm{H}, \mathrm{m}), 7.47-7.43(2 \mathrm{H}, \mathrm{m}), 7.32-7.28(2 \mathrm{H}, \mathrm{m}), 5.55(1 \mathrm{H}, \mathrm{d}, J=$ 
$9.6 \mathrm{~Hz}), 4.59(1 \mathrm{H}, \mathrm{d}, J=9.6 \mathrm{~Hz}), 3.54-3.46(2 \mathrm{H}, \mathrm{m}), 1.53-1.48(2 \mathrm{H}, \mathrm{m}), 1.27-1.14(16 \mathrm{H}$, $\mathrm{m}), 0.85(3 \mathrm{H}, \mathrm{t}, J=7.2 \mathrm{~Hz}) .{ }^{13} \mathrm{C} \mathrm{NMR}\left(150 \mathrm{MHz}, \mathrm{CDCl}_{3}\right) \delta 174.0,172.2,145.4,143.7,134.8$, 130.8, 129.1, 128.1, 126.2, 125.8, 124.2, 123.7, 123.5, 119.7, 111.0, 53.0, 41.5, 39.7, 31.8, 29.5, 29.4, 29.3, 29.2, 28.9, 27.3, 26.5, 22.6, 14.0. IR (ATR): $3310,1175,994,817,723 \mathrm{~cm}^{-1}$; HRMS (ESI) m/z [M+H'] calcd for $\mathrm{C}_{28} \mathrm{H}_{34} \mathrm{~N}_{3} \mathrm{O}_{2}$ : 444.2646, Found: 444.2645.

\section{2-Benzyl-3a,13a-dihydro-1H-benzo[4,5] imidazo[2,1-a]pyrrolo $[3,4-c]$ isoquinoline-}

\section{1,3(2H)-dione (4h):}

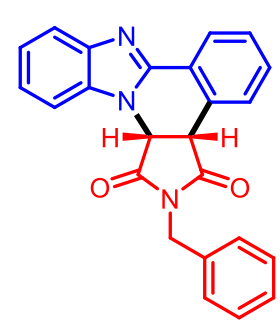

The compound was prepared according to the general procedure and purified by column chromatography $($ EtOAc/hexane $=20: 80)$. Yield: $70 \%$ (133 mg); state: white solid; mp: 226-228 ${ }^{\circ} \mathrm{C} ;{ }^{1} \mathrm{H}$ NMR (600 MHz, $\mathrm{CDCl}_{3}$ ) $\delta 8.38(1 \mathrm{H}, \mathrm{d}, J=7.2 \mathrm{~Hz}), 7.87(1 \mathrm{H}, \mathrm{d}, J=7.2 \mathrm{~Hz}), 7.75(1 \mathrm{H}, \mathrm{d}, J=7.8 \mathrm{~Hz})$, $7.70(1 \mathrm{H}, \mathrm{d}, J=7.2 \mathrm{~Hz}), 7.47-7.41(2 \mathrm{H}, \mathrm{m}), 7.33-7.25(7 \mathrm{H}, \mathrm{m}), 5.60(1 \mathrm{H}$, $\mathrm{d}, J=9.6 \mathrm{~Hz}), 4.67-4.61(3 \mathrm{H}, \mathrm{m}) ;{ }^{13} \mathrm{C} \mathrm{NMR}\left(150 \mathrm{MHz}, \mathrm{CDCl}_{3}\right) \delta 173.6,171.8,145.2,134.5$, 131.0, 129.2, 128.9, 128.8, 128.4, 128.1, 126.1, 126.0, 123.9, 123.7, 119.4, 111.2, 53.1, 43.4, 41.5. IR (ATR): 3301, 2899, 1968, 1362, 997, $758 \mathrm{~cm}^{-1}$; HRMS $\left(\mathrm{EI}^{+}\right) \mathrm{m} / z\left[\mathrm{M}^{+}\right]$calcd for $\mathrm{C}_{24} \mathrm{H}_{17} \mathrm{~N}_{3} \mathrm{O}_{2}$ : 379.1321, Found: 379.1323 .

\section{2,10,11-Trimethyl-3a,13a-dihydro-1H-benzo[4,5]imidazo[2,1-a]pyrrolo[3,4- c]isoquinoline-1,3(2H)-dione (4i):}

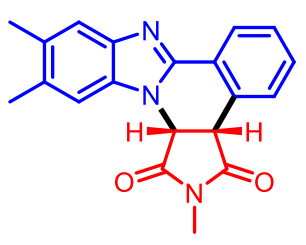

The compound was prepared according to the general procedure and purified by column chromatography $($ EtOAc/hexane $=20: 80)$. Yield: $71 \%$ (118 mg); state: white solid; mp: $240-242{ }^{\circ} \mathrm{C} ;{ }^{1} \mathrm{H}$ NMR $(600 \mathrm{MHz}$, DMSO-d6) $\delta 8.20(1 \mathrm{H}, \mathrm{d}, J=7.2 \mathrm{~Hz}), 7.82(1 \mathrm{H}, \mathrm{d}, J=7.8 \mathrm{~Hz}), 7.55(1 \mathrm{H}$, s), $7.54-7.47(2 \mathrm{H}, \mathrm{m}), 7.46(1 \mathrm{H}, \mathrm{s}), 6.00(1 \mathrm{H}, \mathrm{d}, \mathrm{J}=9.6 \mathrm{~Hz}), 4.87(1 \mathrm{H}, \mathrm{d}, \mathrm{J}=9.6 \mathrm{~Hz}), 2.89$ $(3 \mathrm{H}, \mathrm{s}) 2.37(3 \mathrm{H}, \mathrm{s}), 2.34(3 \mathrm{H}, \mathrm{s}) ;{ }^{13} \mathrm{C}$ NMR $\left(150 \mathrm{MHz}, \mathrm{DMSO}-d_{6}\right) \delta 174.9,173.5,144.5,142.0$, 133.5, 131.6, 131.2, 130.2, 128.5, 128.1, 127.7, 124.7, 124.4, 119.0, 111.8, 52.8, 41.4, 25.1, 20.3, 19.9. IR (ATR): 3326, 2922, 1706, 1218, 1081, $709 \mathrm{~cm}^{-1}$; HRMS (EI $\left.{ }^{+}\right) \mathrm{m} / z\left[\mathrm{M}^{+}\right]$calcd for $\mathrm{C}_{20} \mathrm{H}_{17} \mathrm{~N}_{3} \mathrm{O}_{2}$ : 331.1321, Found: 331.1323 . 


\section{0,11-Dichloro-2-methyl-3a,13a-dihydro-1H-benzo[4,5]imidazo[2,1-a]pyrrolo[3,4-}

c]isoquinoline-1,3(2H)-dione $(4 \mathbf{j})$ :

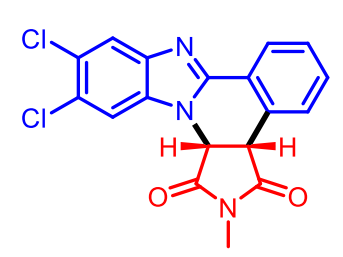

The compound was prepared according to the general procedure and purified by column chromatography (EtOAc/hexane = 20: 80). Yield: 69\% (128 mg); State: white solid; mp: 280-282 ${ }^{\circ} \mathrm{C} ;{ }^{1} \mathrm{H}$ NMR (600 MHz, DMSO- $\left.d_{6}\right) \delta 8.23(1 \mathrm{H}, \mathrm{d}, J=6.6 \mathrm{~Hz}), 8.13(1 \mathrm{H}, \mathrm{s}), 7.98(1 \mathrm{H}, \mathrm{s}), 7.85$ $(1 \mathrm{H}, \mathrm{d}, J=7.8 \mathrm{~Hz}), 7.60(1 \mathrm{H}, \mathrm{td}, J=7.2,1.2 \mathrm{~Hz}), 7.52(1 \mathrm{H}, \mathrm{t}, J=7.2 \mathrm{~Hz}), 6.08(1 \mathrm{H}, \mathrm{d}, J=9.6$ $\mathrm{Hz}), 4.91(1 \mathrm{H}, \mathrm{d}, J=9.6 \mathrm{~Hz}), 2.89(3 \mathrm{H}, \mathrm{s}) ;{ }^{13} \mathrm{C}$ NMR (150 MHz, DMSO-d $\left.d_{6}\right) \delta 174.6,173.2$, $147.9,142.9,134.5,131.1,128.5,128.5,128.2,125.2$, 125.1, 125.0, 123.4, 120.0, 113.8, 52.9, 41.1, 25.3; IR (ATR): 3395, 2922, 1709, 1549, 1024, $757 \mathrm{~cm}^{-1}$; HRMS $\left(\mathrm{EI}^{+}\right) \mathrm{m} / z\left[\mathrm{M}^{+}\right]$calcd for $\mathrm{C}_{18} \mathrm{H}_{11} \mathrm{Cl}_{2} \mathrm{~N}_{3} \mathrm{O}_{2}$ : 371.0228, Found: 371.0229 .

\section{5-(tert-Butyl)-2-phenyl-3a,13a-dihydro-1H-benzo[4,5]imidazo[2,1-a]pyrrolo[3,4- $c$ ]isoquinoline-1,3(2H)-dione (4k) :}

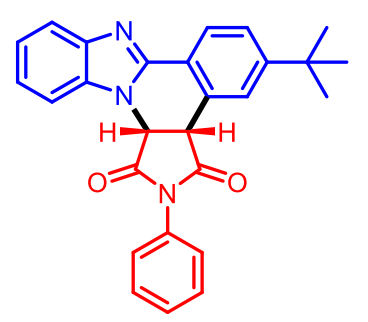

The compound was prepared according to the general procedure and purified by column chromatography $($ EtOAc/hexane $=20: 80)$. Yield: $68 \%$ (144 mg); state: orange solid; mp: 230-232 ${ }^{\circ} \mathrm{C} ;{ }^{1} \mathrm{H}$ NMR (600 $\left.\mathrm{MHz}, \mathrm{DMSO}-d_{6}\right) \delta 8.23(1 \mathrm{H}, \mathrm{d}, J=8.4 \mathrm{~Hz}), 7.91(1 \mathrm{H}, \mathrm{s}), 7.80(1 \mathrm{H}, \mathrm{d}$, $J=7.8 \mathrm{~Hz}), 7.70(1 \mathrm{H}, \mathrm{d}, J=8.4 \mathrm{~Hz}), 7.61(1 \mathrm{H}, \mathrm{dd}, J=7.8,1.2 \mathrm{~Hz})$, $7.49(2 \mathrm{H}, \mathrm{t}, J=7.2 \mathrm{~Hz}), 7.43(1 \mathrm{H}, \mathrm{t}, J=7.2 \mathrm{~Hz}), 7.33-7.30(3 \mathrm{H}, \mathrm{m}), 7.29-7.25(1 \mathrm{H}, \mathrm{m}), 6.30$ $(1 \mathrm{H}, \mathrm{d}, J=10.2 \mathrm{~Hz}), 5.07(1 \mathrm{H}, \mathrm{d}, J=10.2 \mathrm{~Hz}), 1.34(9 \mathrm{H}, \mathrm{s}) ;{ }^{13} \mathrm{C}$ NMR $\left(150 \mathrm{MHz}, \mathrm{DMSO}-d_{6}\right)$ $\delta 174.0,172.6,153.4,145.7,143.5,135.1,131.7,129.0,128.7,126.8,125.9,125.0,124.6$, 122.7, 122.6, 121.9, 118.9, 111.7, 53.2, 41.6, 34.8, 30.8. IR (ATR): 2960, 1721, 1430, 1179, $750 \mathrm{~cm}^{-1}$; HRMS $\left(\mathrm{EI}^{+}\right) \mathrm{m} / z,\left[\mathrm{M}^{+}\right]$calcd for $\mathrm{C}_{27} \mathrm{H}_{23} \mathrm{~N}_{3} \mathrm{O}_{2}$ : 421.1790 , Found: 421.1793.

\section{5-Chloro-2-phenyl-3a,13a-dihydro-1H-benzo[4,5]imidazo[2,1-a]pyrrolo[3,4-c] isoquinoline-1,3(2H)-dione (4I):}

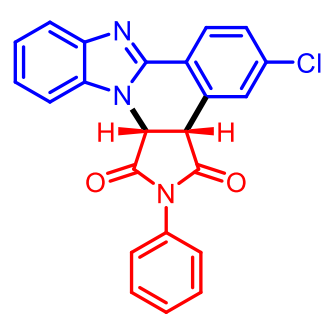

The compound was prepared according to the general procedure and purified by column chromatography $($ EtOAc/hexane $=20: 80)$. Yield: $71 \%$ (141 mg); state: white solid; mp: $270-272{ }^{\circ} \mathrm{C} ;{ }^{1} \mathrm{H}$ NMR $(600 \mathrm{MHz}$, DMSO- $\left.d_{6}\right) \delta 8.29(1 \mathrm{H}, \mathrm{d}, J=8.4 \mathrm{~Hz}), 7.88(1 \mathrm{H}, \mathrm{s}), 7.83(1 \mathrm{H}, \mathrm{d}, J=7.8$ 
$\mathrm{Hz}), 7.72(1 \mathrm{H}, \mathrm{d}, J=7.8 \mathrm{~Hz}), 7.61(1 \mathrm{H}, \mathrm{dd}, J=8.4,1.2 \mathrm{~Hz}), 7.49(2 \mathrm{H}, \mathrm{t}, J=7.2 \mathrm{~Hz}), 7.43(1 \mathrm{H}$, t, $J=7.8 \mathrm{~Hz}), 7.33(3 \mathrm{H}, \mathrm{t}, J=7.8 \mathrm{~Hz}), 7.29(1 \mathrm{H}, \mathrm{t}, J=7.2 \mathrm{~Hz}), 6.27(1 \mathrm{H}, \mathrm{d}, J=10.1 \mathrm{~Hz}), 5.10$ $(1 \mathrm{H}, \mathrm{d}, J=10.2 \mathrm{~Hz}) ;{ }^{13} \mathrm{C}$ NMR $\left(150 \mathrm{MHz}, \mathrm{DMSO}-d_{6}\right) \delta 173.6,172.4,145.0,143.3,135.08$, $134.8,131.7,130.1,128.9,128.8,128.6,127.8,127.0,126.8,123.5,123.05,122.8,119.1$, 112.1, 53.0, 41.1; IR (ATR): 3404, 1559, 1046, 998, $764 \mathrm{~cm}^{-1}$; HRMS (ESI) $m / z$ [M+H'] calcd for $\mathrm{C}_{23} \mathrm{H}_{15} \mathrm{ClN}_{3} \mathrm{O}_{2}$ : 400.0847, Found: 400.0844 .

\section{2-Phenyl-5-(trifluoromethyl)-3a,13a-dihydro-1 $H$-benzo[4,5]imidazo[2,1-a]pyrrolo[3,4- c]isoquinoline-1,3(2H)-dione $(4 \mathrm{~m})$}

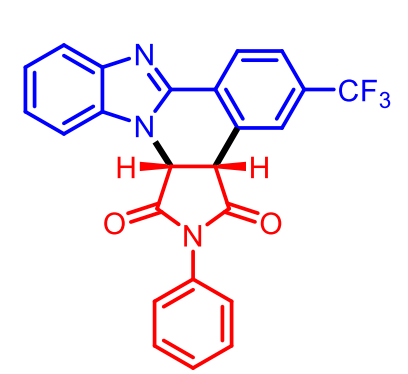

The compound was prepared according to the general procedure and purified by column chromatography (EtOAc/hexane $=25: 75)$. Yield: 69\% (150 mg); state: white solid; mp: $190-192{ }^{\circ} \mathrm{C} ; \quad{ }^{1} \mathrm{H}$ NMR (600 MHz, DMSO-d 6$) \delta 8.49(1 \mathrm{H}, \mathrm{d}, J=8.2 \mathrm{~Hz}), 8.18(1 \mathrm{H}, \mathrm{d}, J=1.2 \mathrm{~Hz})$, $7.88(2 \mathrm{H}, \mathrm{d}, J=7.8 \mathrm{~Hz}), 7.75(1 \mathrm{H}, \mathrm{d}, J=7.8 \mathrm{~Hz}), 7.50-7.47(2 \mathrm{H}$, m), $7.44-7.41(1 \mathrm{H}, \mathrm{m}), 7.38-7.30(4 \mathrm{H}, \mathrm{m}), 6.29(1 \mathrm{H}, \mathrm{d}, J=10.2$ $\mathrm{Hz}), 5.19(1 \mathrm{H}, \mathrm{d}, J=10.2 \mathrm{~Hz}) ;{ }^{13} \mathrm{C}$ NMR $\left(150 \mathrm{MHz}, \mathrm{DMSO}-d_{6}\right) \delta 173.7,172.3,144.5,143.3$, $135.1,131.8,129.9,129.7,129.1,128.8,128.8,128.4,127.0,125.7,125.1\left(\mathrm{C}-\mathrm{F},{ }^{2} J \mathrm{C}-\mathrm{F}=3.9\right.$ Hz), $125.0\left(\mathrm{C}-\mathrm{F},{ }^{2} J \mathrm{C}-\mathrm{F}=3.9 \mathrm{~Hz}\right), 124.8\left(\mathrm{C}-\mathrm{F},{ }^{1} \mathrm{JC}-\mathrm{F}=270.9 \mathrm{~Hz}\right), 123.4,123.1,123.0(\mathrm{C}-\mathrm{F}$, $\left.{ }^{1} \mathrm{JC}-\mathrm{F}=270.9 \mathrm{~Hz}\right), 119.3,112.5,53.0,41.0 ;{ }^{19} \mathrm{~F}$ NMR $\left(565 \mathrm{~Hz}, \mathrm{DMSO}-d_{6}\right) \delta-61.4(1 \mathrm{~F}) . \mathrm{IR}$ (ATR): 1715, 1600, 1546, 1376, $1131 \mathrm{~cm}^{-1}$; HRMS (ESI) $\mathrm{m} / z\left[\mathrm{M}+\mathrm{H}^{+}\right]$calcd for $\mathrm{C}_{24} \mathrm{H}_{15} \mathrm{~F}_{3} \mathrm{~N}_{3} \mathrm{O}_{2}$ : 434.1111, Found: 434.1107.

\section{3-(2-(1H-Benzo[d]imidazol-2-yl)phenyl)-1-phenylpyrrolidine-2,5-dione (5a):}

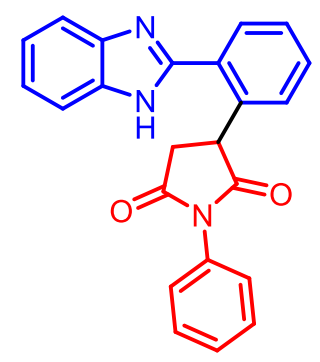

The compound was prepared according to the general procedure and purified by column chromatography (EtOAc/hexane $=20: 80)$. Yield: $80 \%$ (147 mg); state: white solid; mp: 222-224 ${ }^{\circ} \mathrm{C}$; ${ }^{1} \mathrm{H}$ NMR $(600 \mathrm{MHz}$, DMSO- $\left.d_{6}\right) \delta 7.83(3 \mathrm{H}, \mathrm{d}, J=6.6 \mathrm{~Hz}), 7.66(3 \mathrm{H}, \mathrm{d}, J=5.4 \mathrm{~Hz}), 7.56(3 \mathrm{H}$, t, $J=7.8 \mathrm{~Hz}), 7.49-7.35(5 \mathrm{H}, \mathrm{m}), 6.14(1 \mathrm{H}, \mathrm{dd}, J=9.6,7.2 \mathrm{~Hz}), 3.64$ $(1 \mathrm{H}, \mathrm{dd}, J=18,9.6 \mathrm{~Hz}), 3.52(1 \mathrm{H}, \mathrm{dd}, J=18,6.6 \mathrm{~Hz}) ;{ }^{13} \mathrm{C} \mathrm{NMR}(150$ MHz, DMSO- $\left.d_{6}\right) \delta 173.0,172.6,153.9,142.4,133.2,132.0,130.2,129.6,129.1,129.0,128.7$, 127.0, 123.5, 122.8, 119.7, 110.8, 54.1, 34.5; IR (ATR): 3334, 2952, 1720, 1363, $712 \mathrm{~cm}^{-1}$; HRMS (ESI) $m / z\left[\mathrm{M}+\mathrm{H}^{+}\right]$calcd for $\mathrm{C}_{23} \mathrm{H}_{18} \mathrm{~N}_{3} \mathrm{O}_{2}$ : 368.1394, Found: 368.1390 . 
2-(5-(2,5-dioxo-1-phenylpyrrolidin-3-yl)-6-oxo-5,6-dihydrobenzo[4,5]imidazo[2,1a]isoquinolin-5-yl-1,2,3,4-d4)-N-phenylacetamide (3a-D')

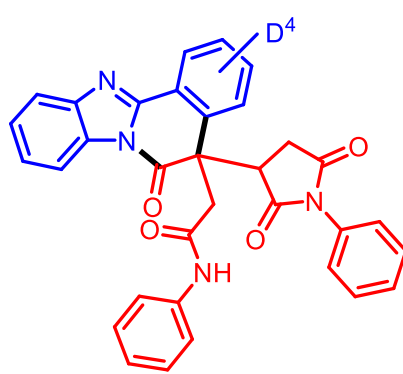

${ }^{1} \mathrm{H}$ NMR (600 MHz, DMSO- $\left.d_{6}\right) \delta 10.25(1 \mathrm{H}, \mathrm{s}), 8.39(1 \mathrm{H}, \mathrm{s}) 8.27$ $(1 \mathrm{H}, \mathrm{dd}, J=7.2,1.8 \mathrm{~Hz}), 7.87(1 \mathrm{H}, \mathrm{dd}, J=6.6,1.2 \mathrm{~Hz}), 7.52-7.47$ $(2 \mathrm{H}, \mathrm{m}), 7.41-7.36(3 \mathrm{H}, \mathrm{m}), 7.24(2 \mathrm{H}, \mathrm{d}, J=7.8 \mathrm{~Hz}), 7.15-7.11(2 \mathrm{H}$, m), $6.98-6.91(3 \mathrm{H}, \mathrm{m}), 4.08(1 \mathrm{H}, \mathrm{d}, J=16.8 \mathrm{~Hz}), 3.95(1 \mathrm{H}, \mathrm{dd}, J=$ 9, $4.2 \mathrm{~Hz}), 3.87(1 \mathrm{H}, \mathrm{d}, J=16.8 \mathrm{~Hz}), 3.06(1 \mathrm{H}, \mathrm{dd}, J=18.0,9.0 \mathrm{~Hz})$, $2.64-2.57(1 \mathrm{H}, \mathrm{m})$; HRMS (ESI) $\mathrm{m} / z\left[\mathrm{M}+\mathrm{H}^{+}\right]$calcd for $\mathrm{C}_{33} \mathrm{H}_{21} \mathrm{D}$ ${ }_{3} \mathrm{~N}_{3} \mathrm{O}_{2}$ : 544.2058, Found: 544.2057. 
Copies of ${ }^{1} \mathrm{H}$ NMR and ${ }^{13} \mathrm{C}$ NMR spectra of starting material 20

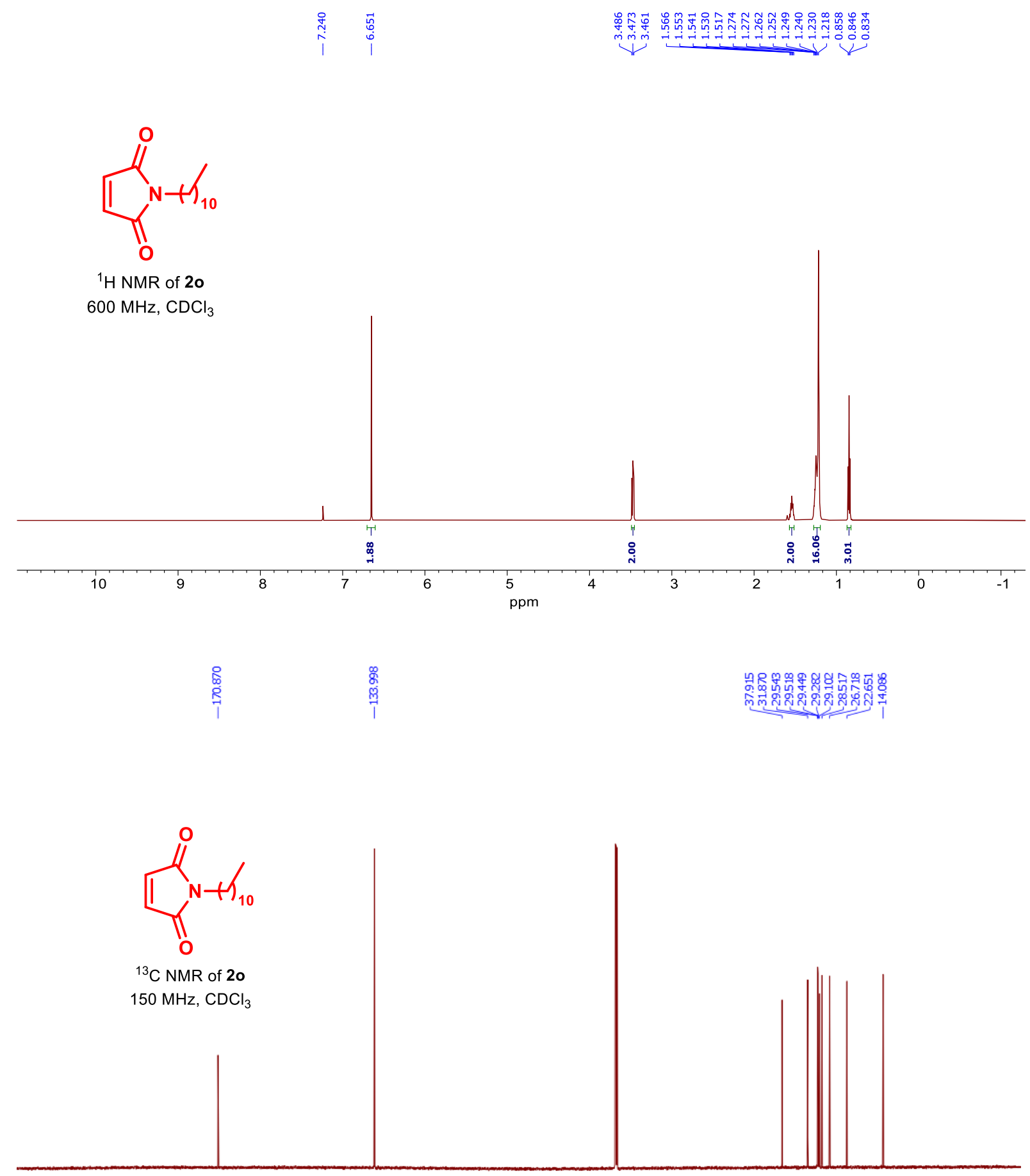

${ }_{150 \mathrm{MHz},}^{{ }_{13} \mathrm{C} \mathrm{NMR}}$

$160-140$

120

100

$80-60$

40

20

0 


\section{Copies of ${ }^{1} \mathrm{H}$ NMR and ${ }^{13} \mathrm{C}$ NMR spectra of the synthesized compounds}
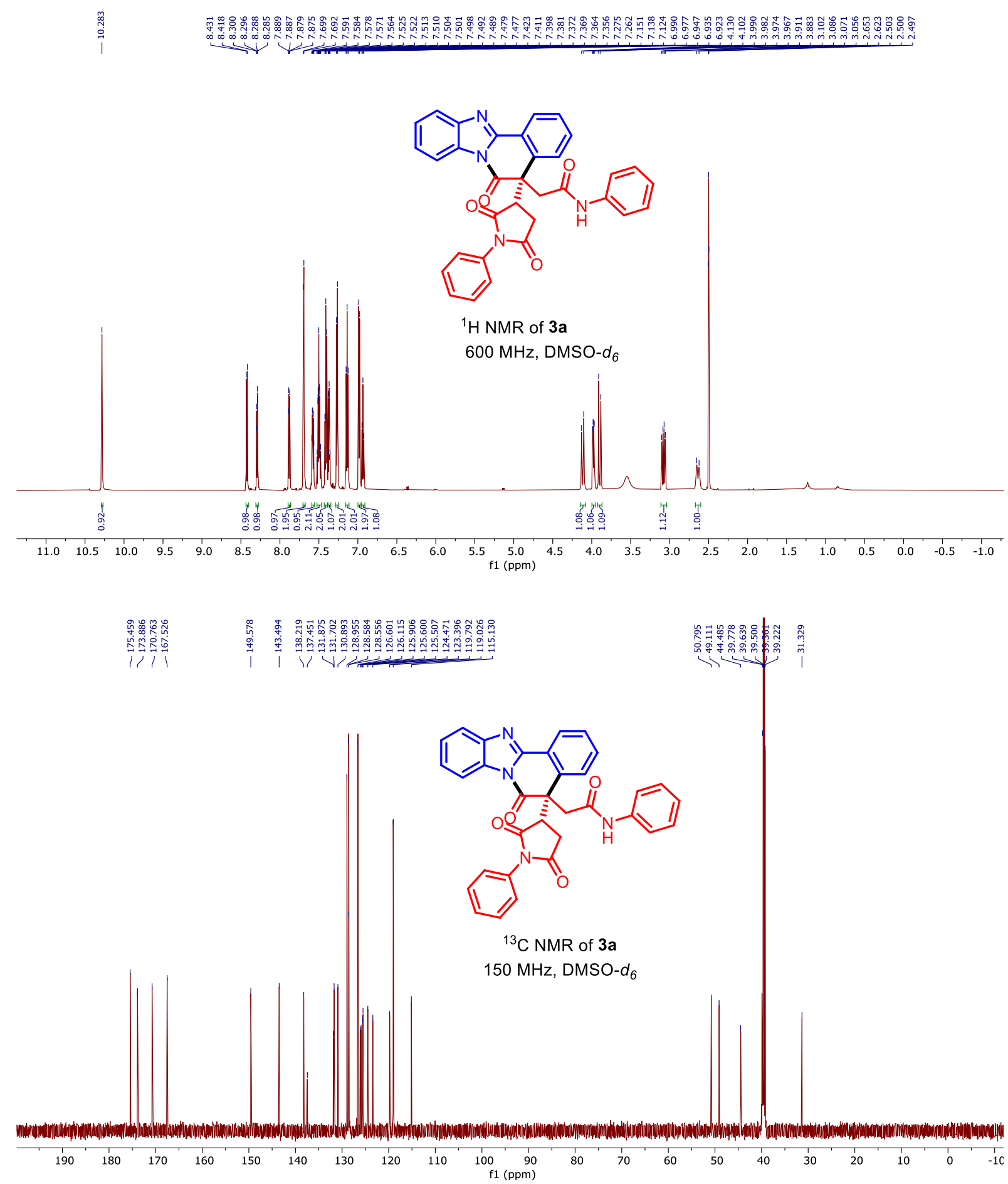

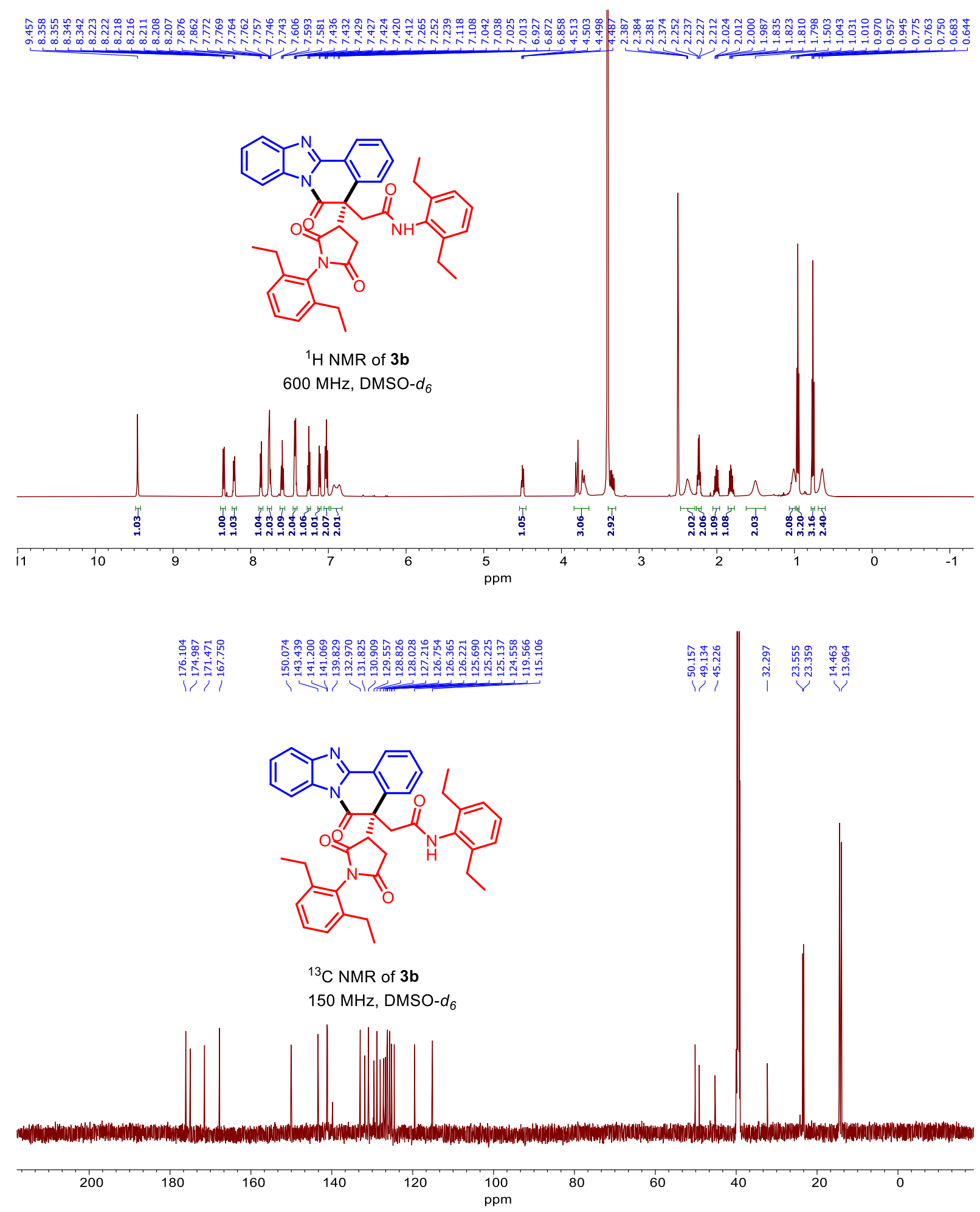


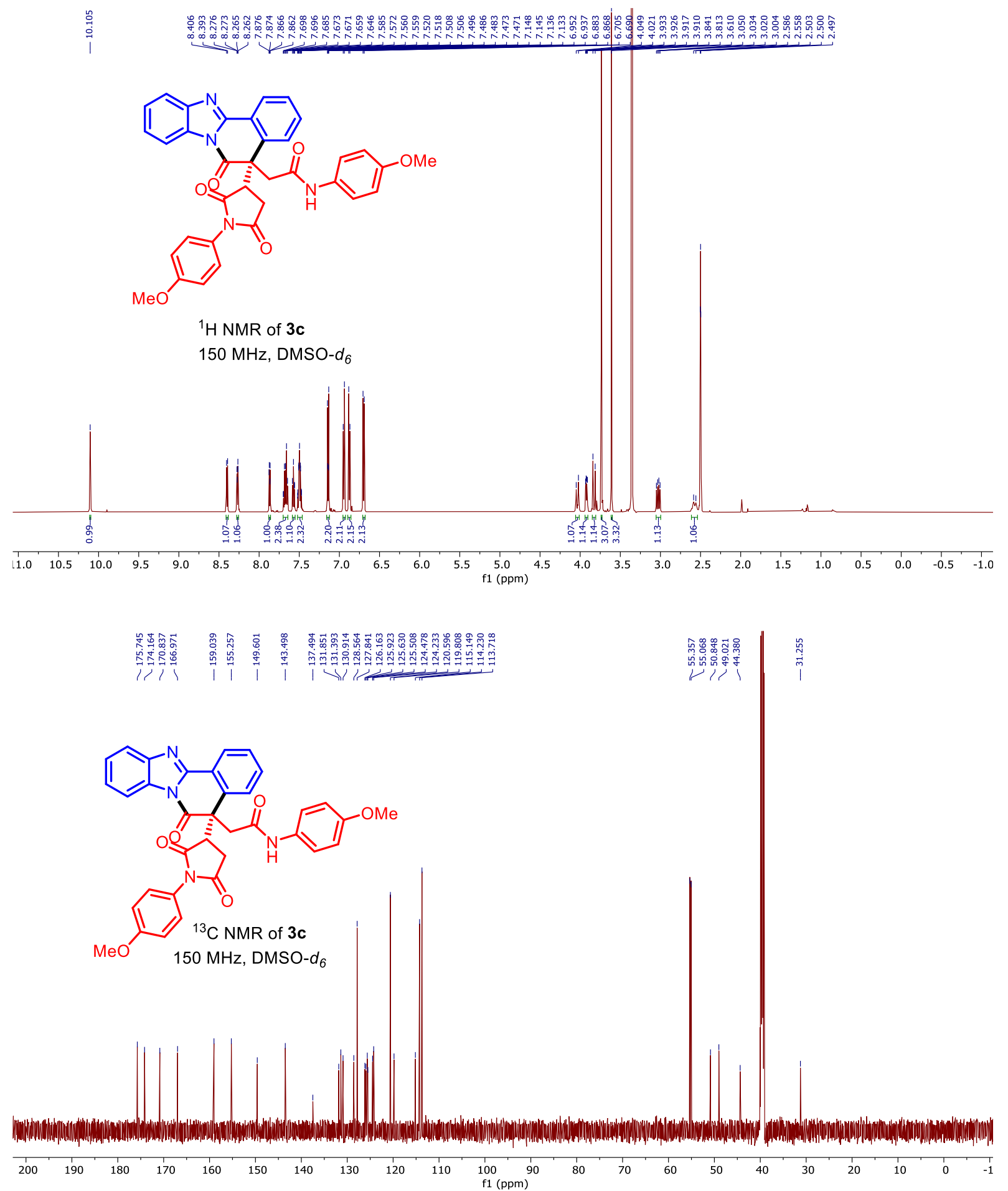




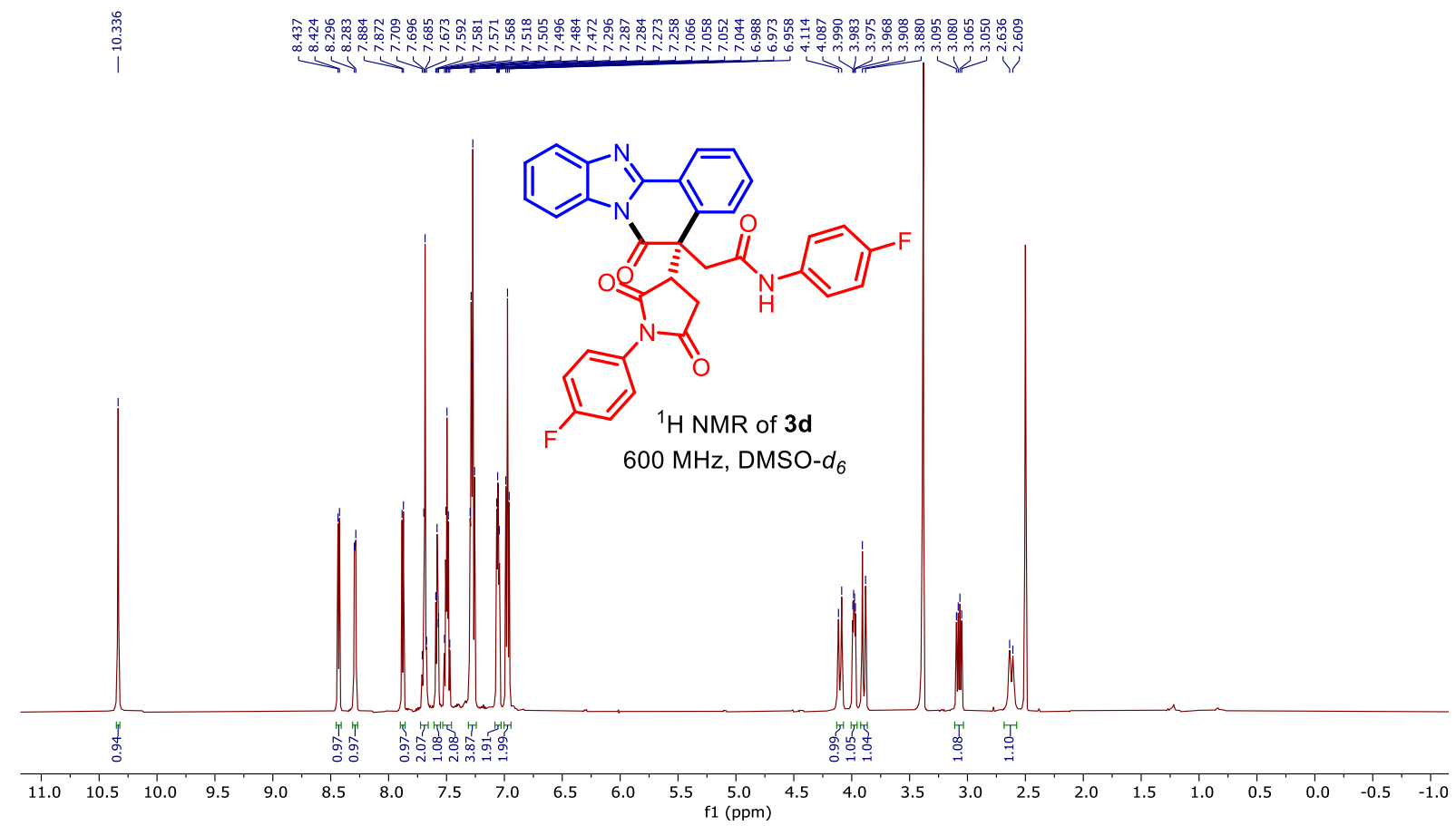

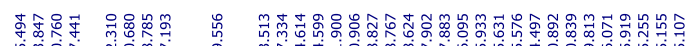

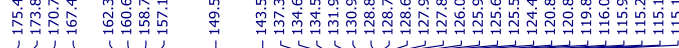

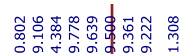

1111111101 1

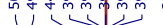

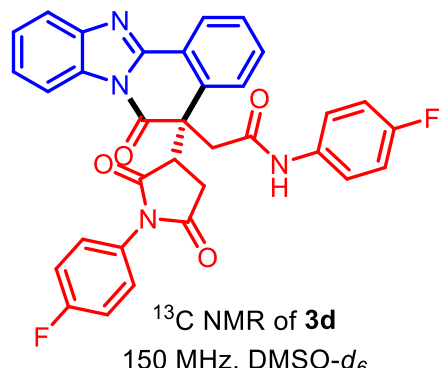

$150 \mathrm{MHz}, \mathrm{DMSO}-\mathrm{d}_{6}$

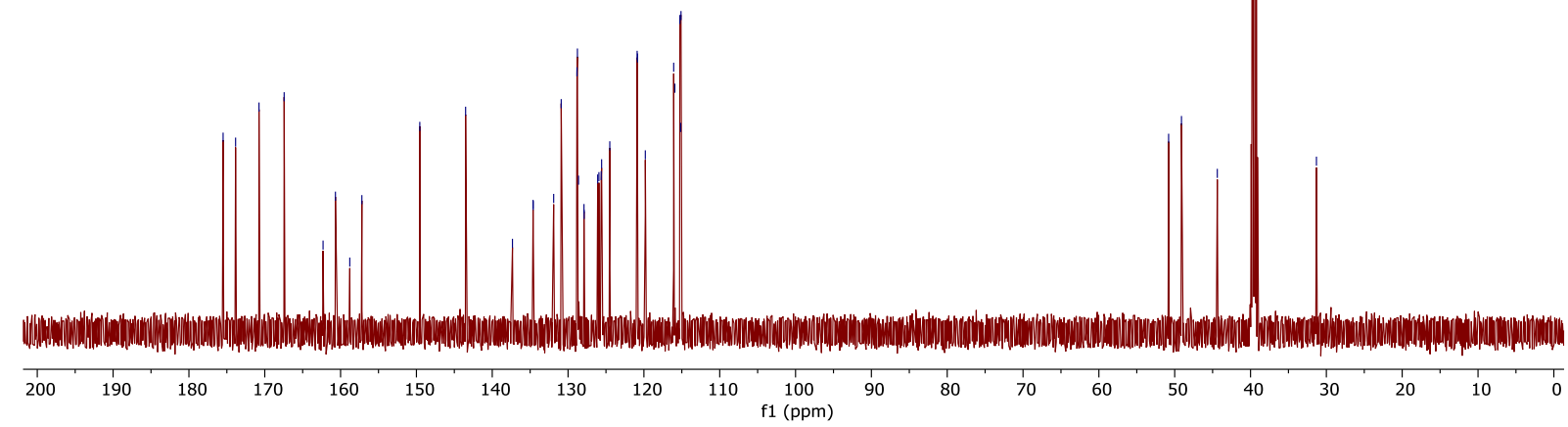




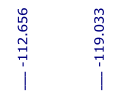
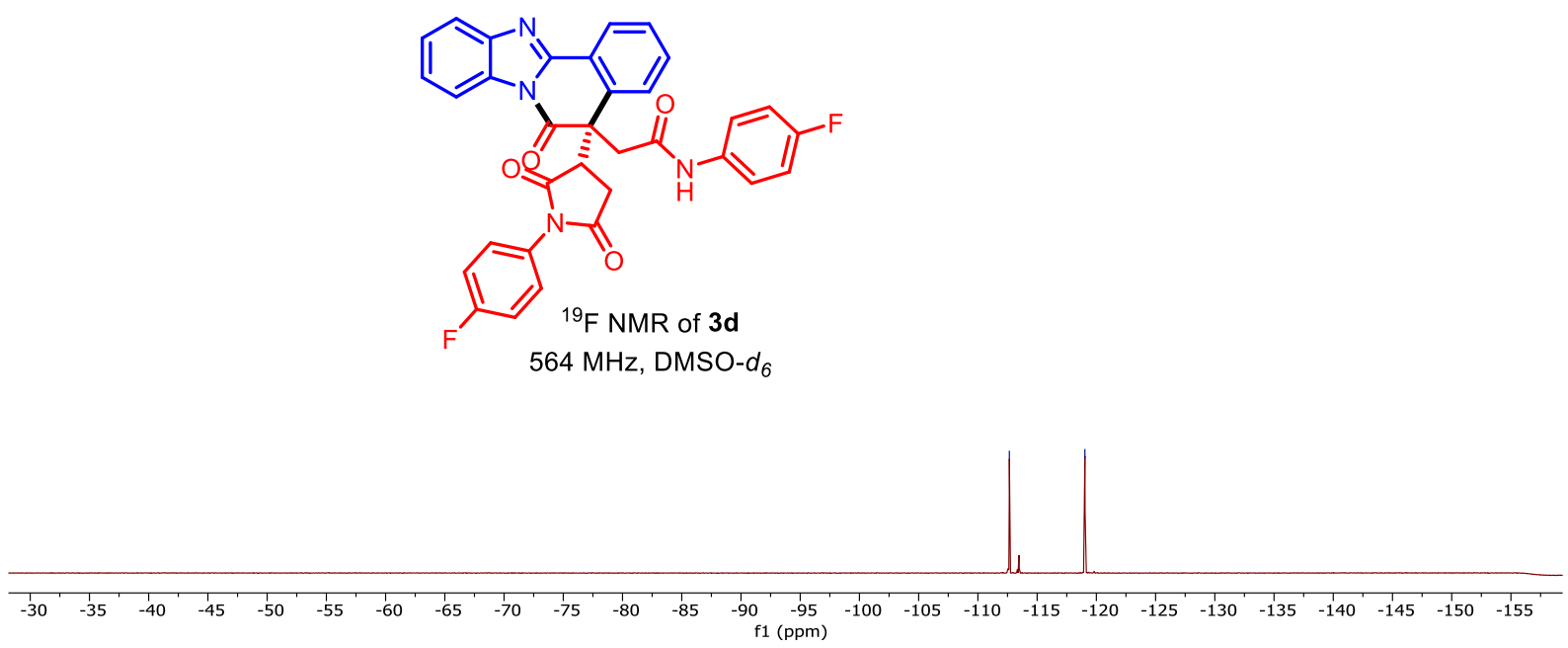

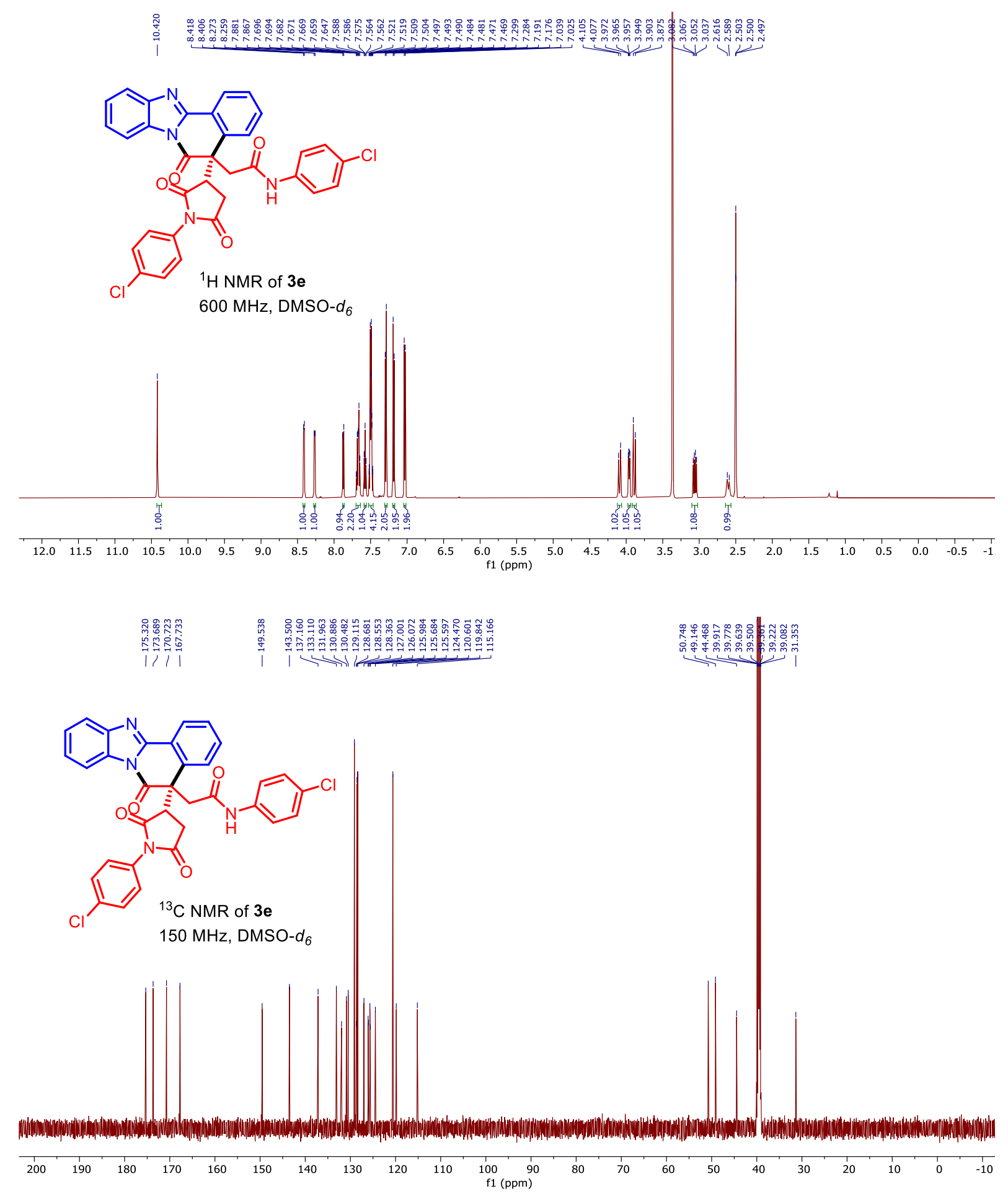

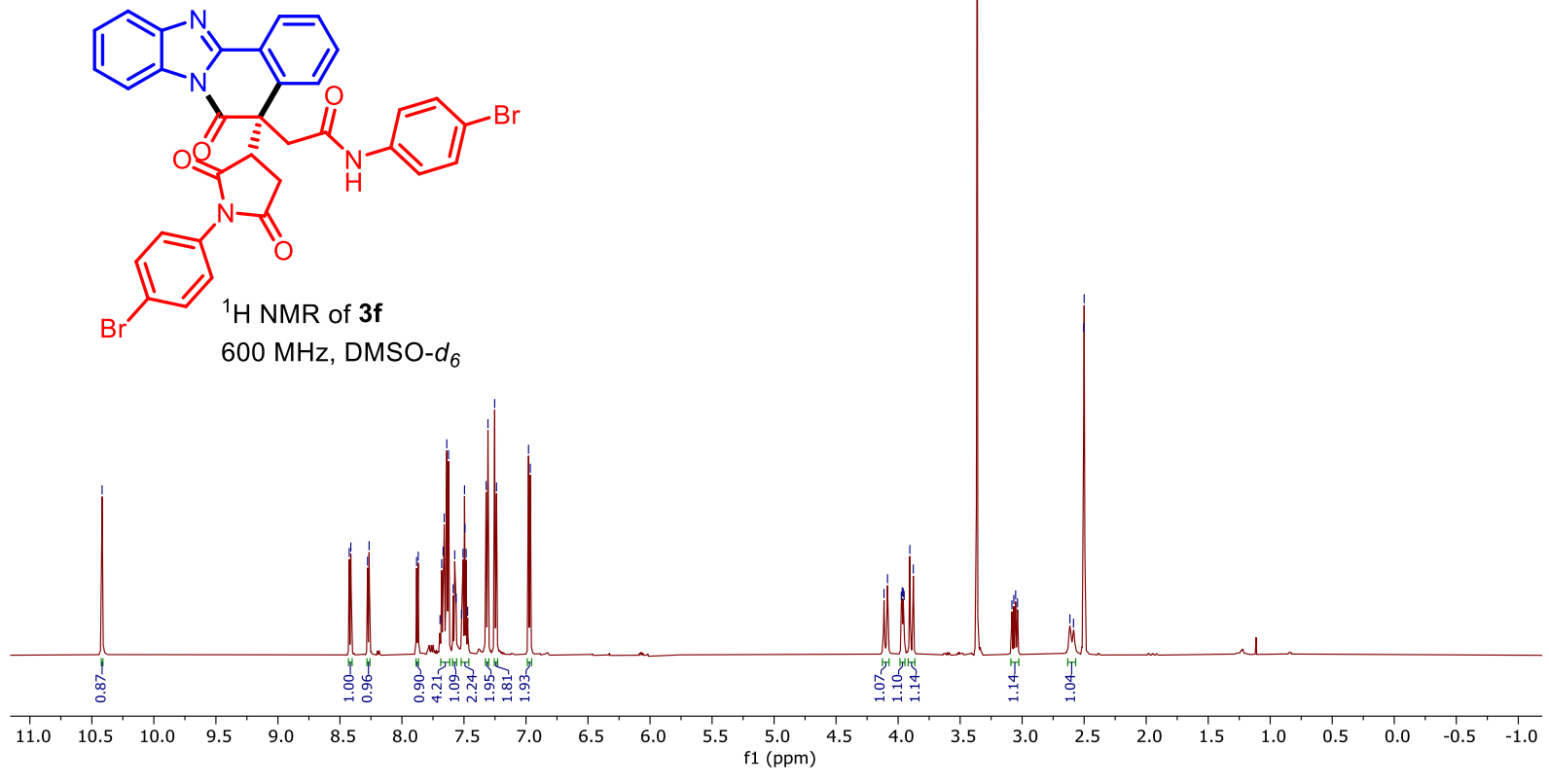

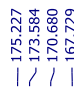

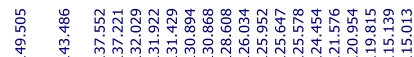

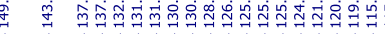

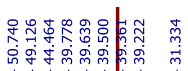

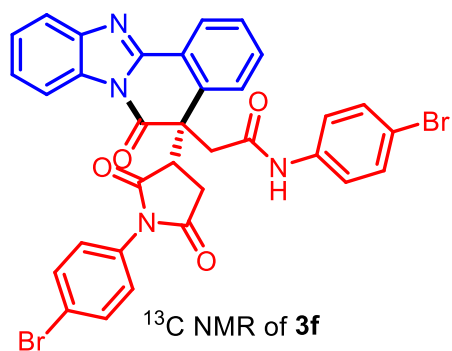

$150 \mathrm{MHz}$, DMSO- $d_{6}$

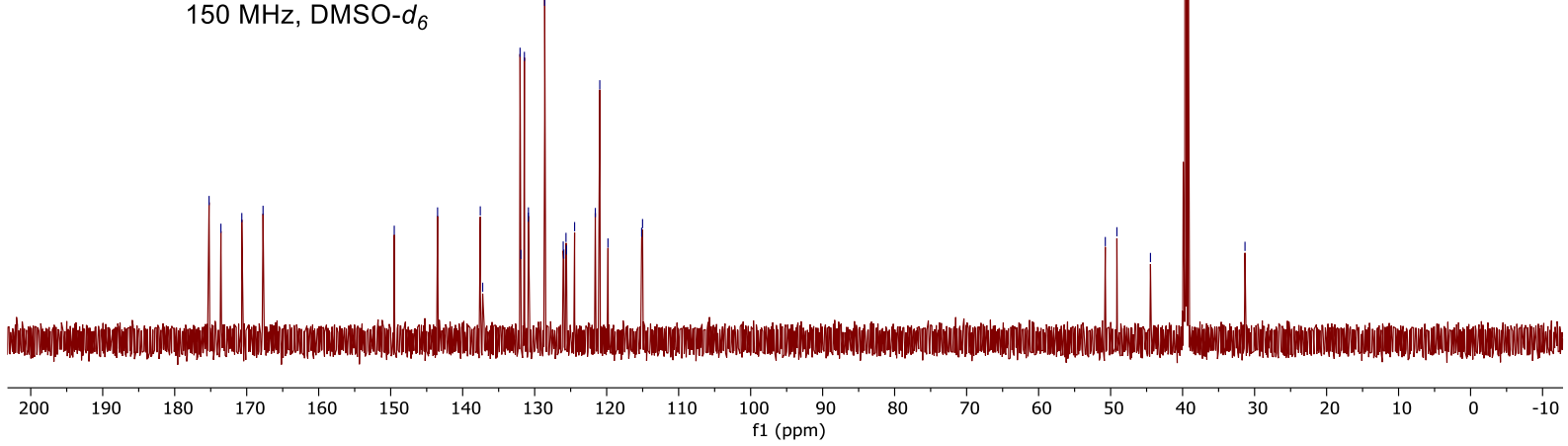



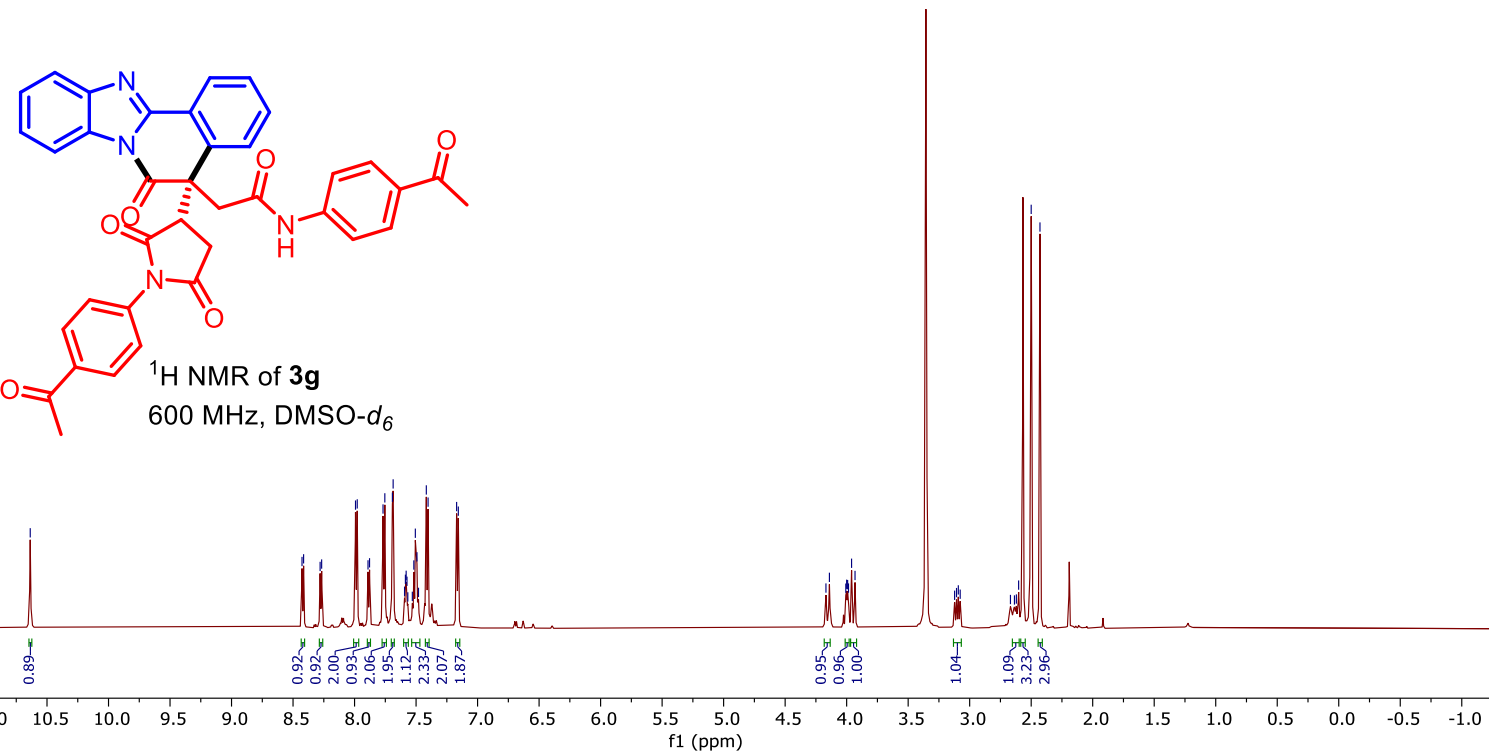

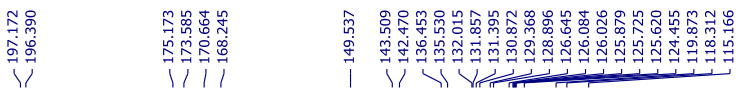

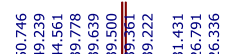

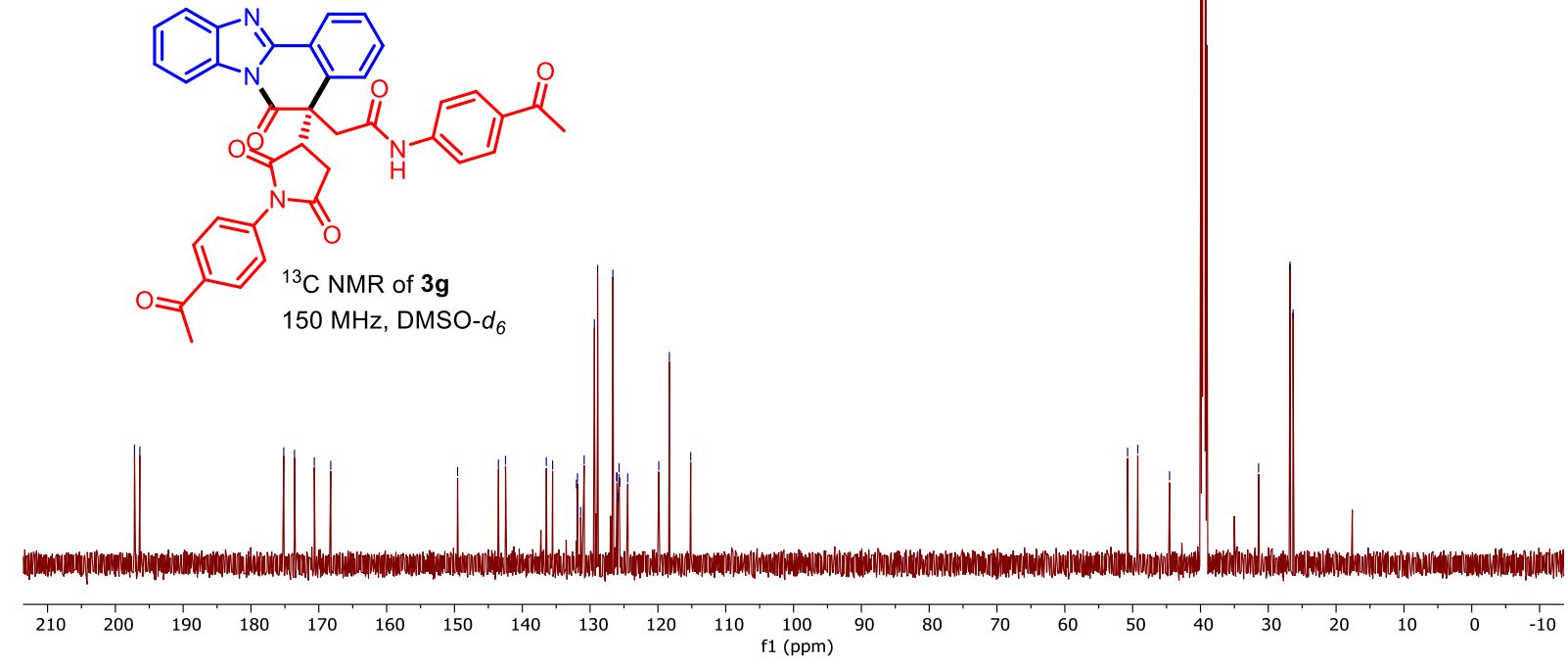



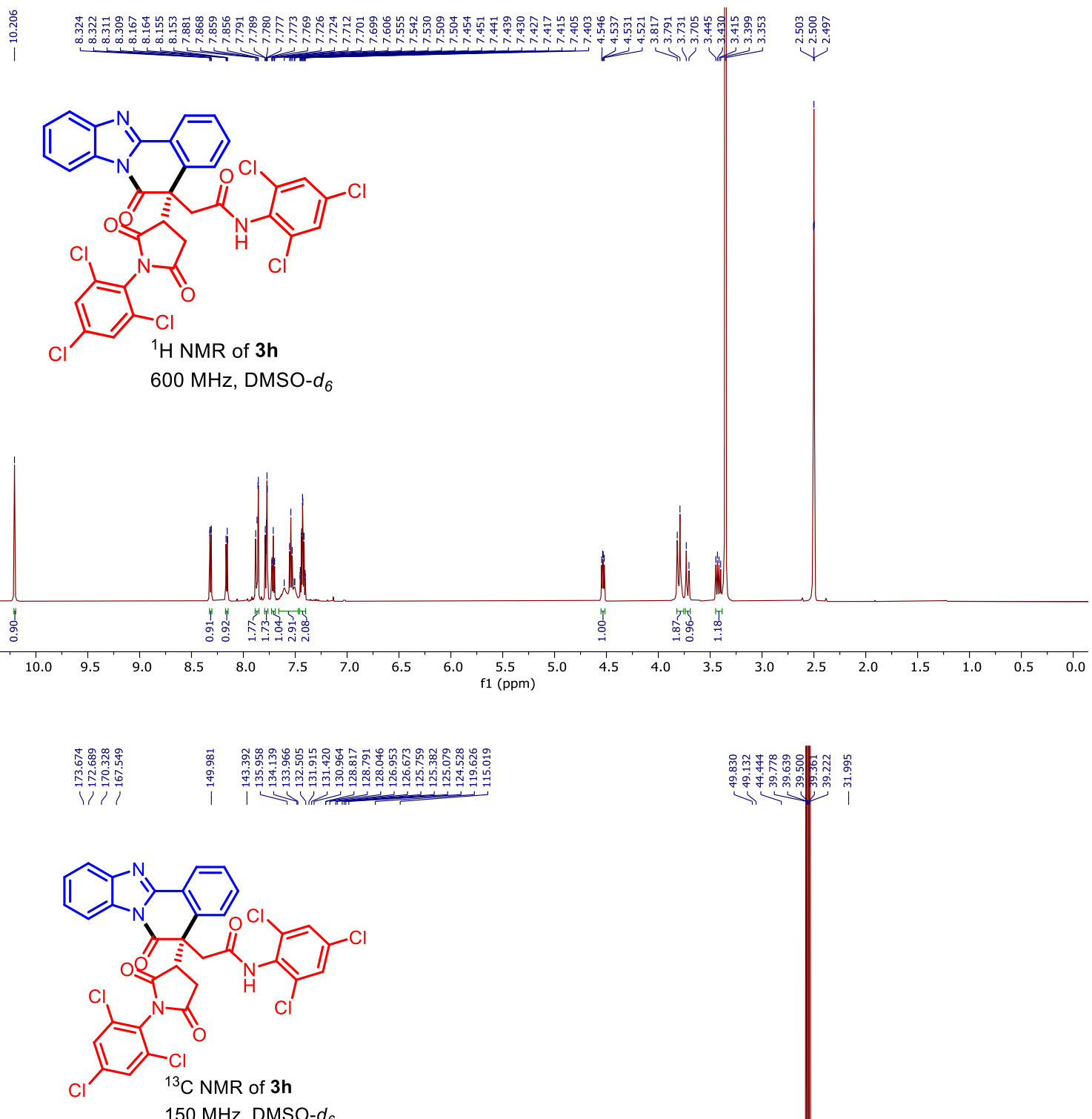

$150 \mathrm{MHz}$, DMSO- $d_{6}$

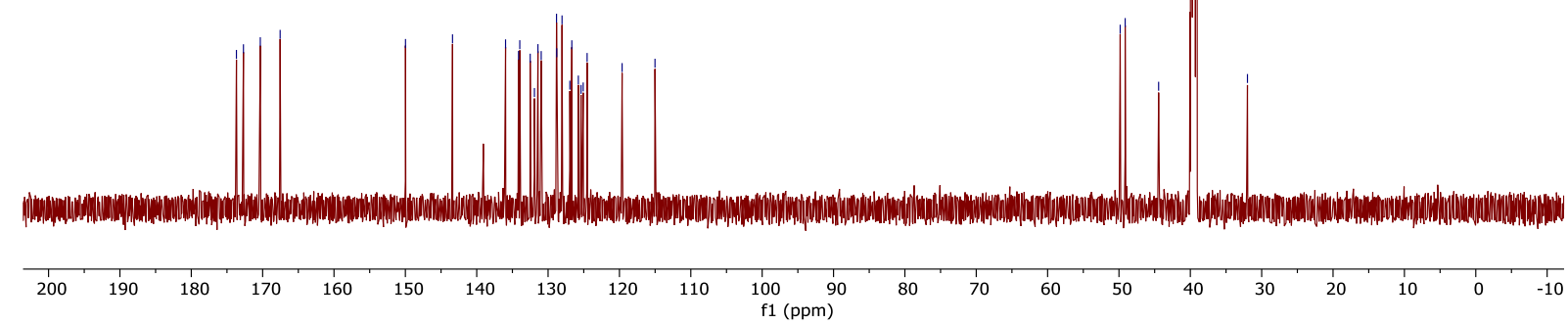



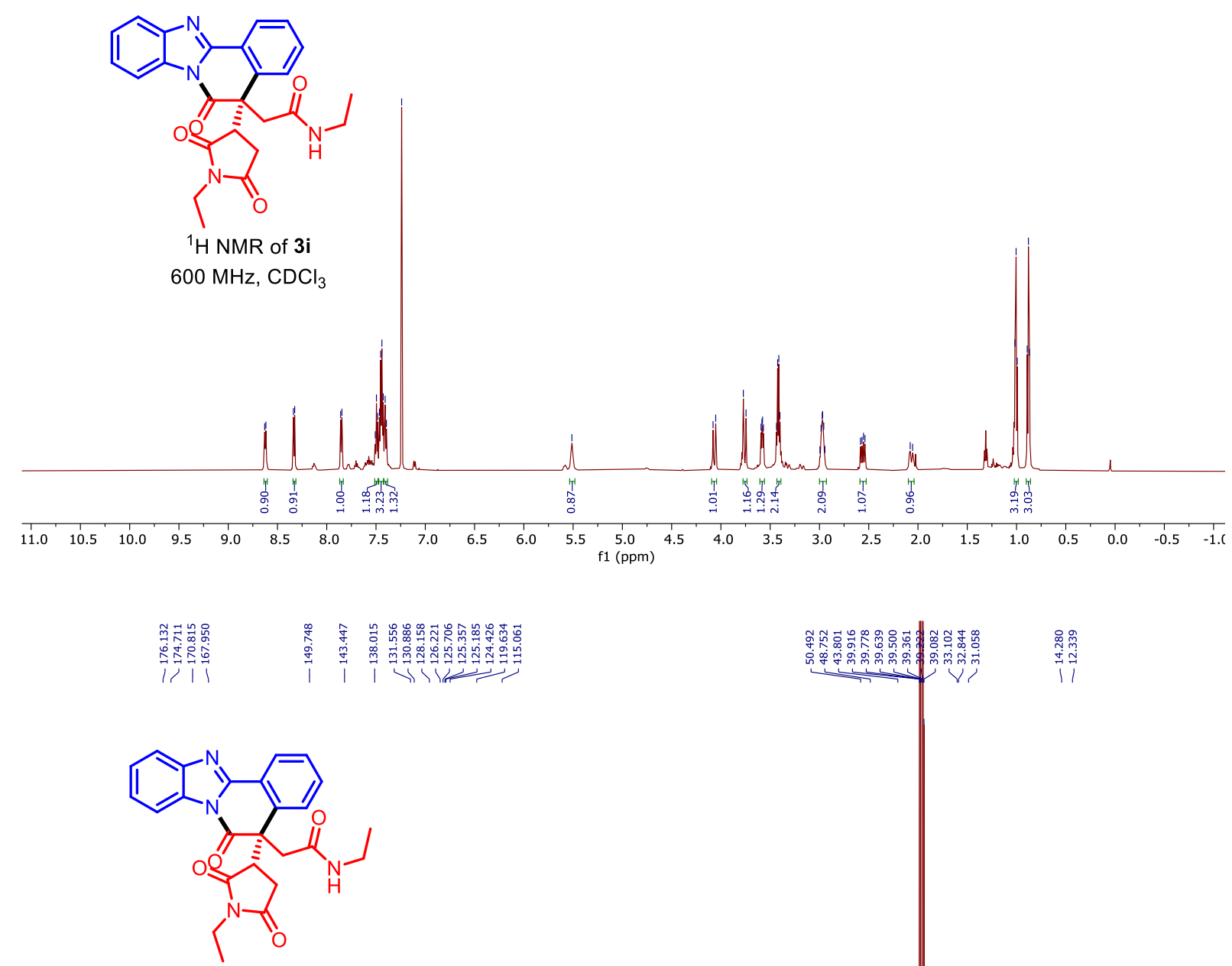

${ }^{13} \mathrm{C}$ NMR of $3 \mathbf{i}$

$150 \mathrm{MHz}, \mathrm{DMSO}-\mathrm{d}_{6}$

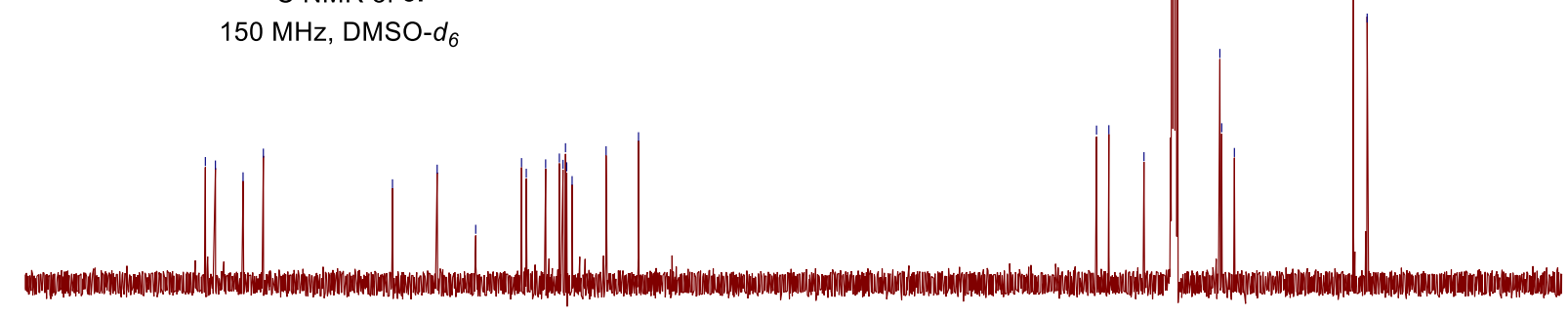

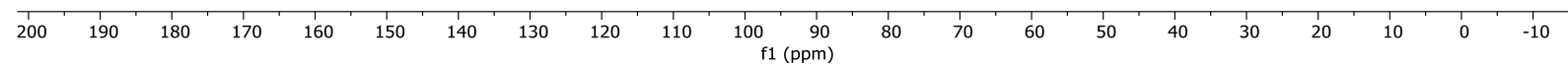




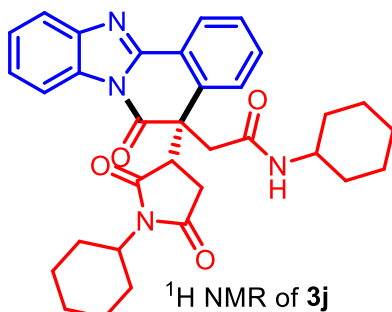

$600 \mathrm{MHz}$, DMSO-d

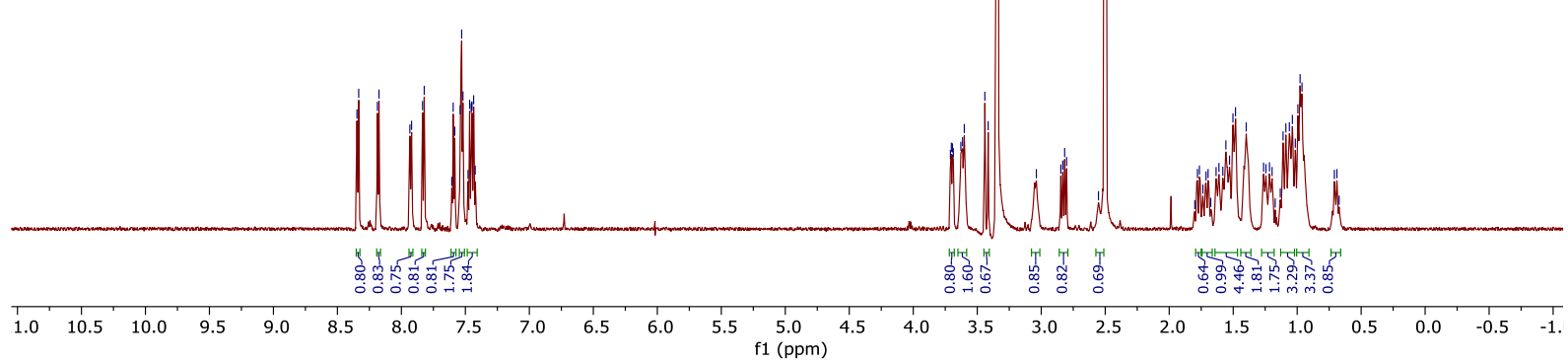

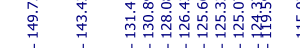

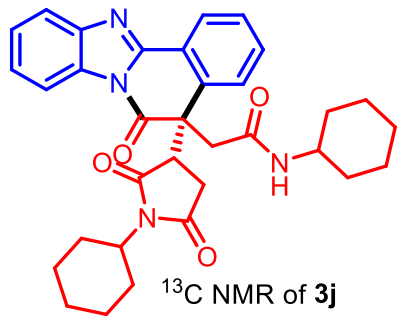

$150 \mathrm{MHz}, \mathrm{DMSO}-d_{6}$

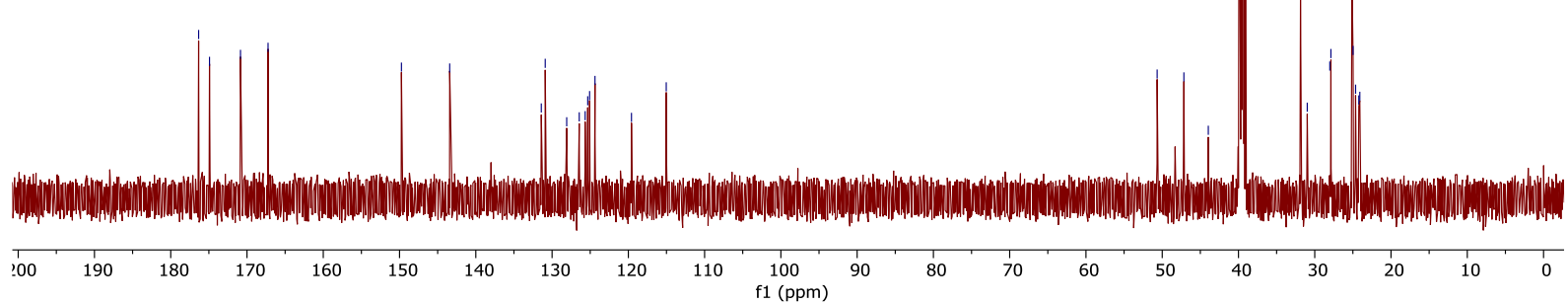



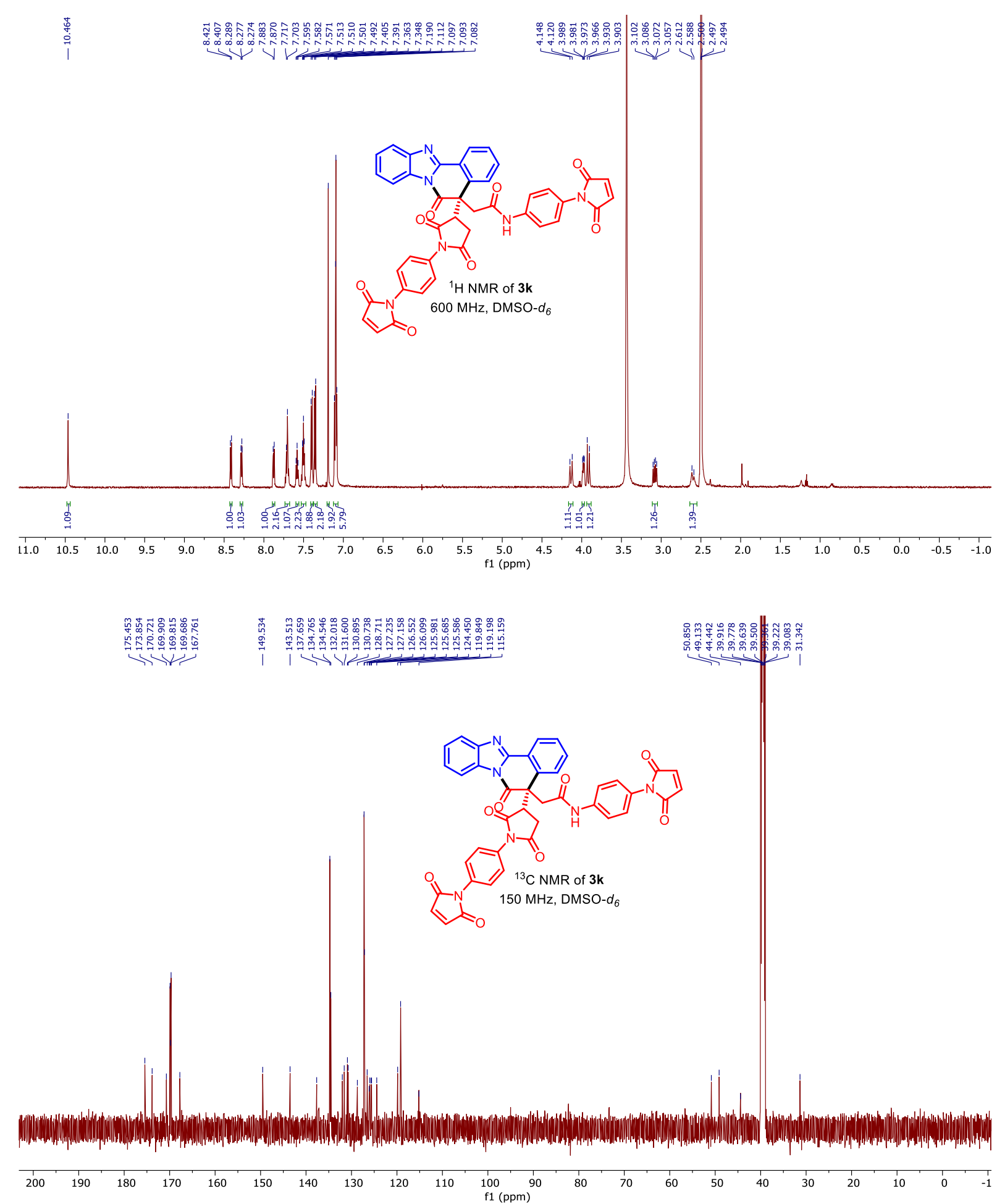

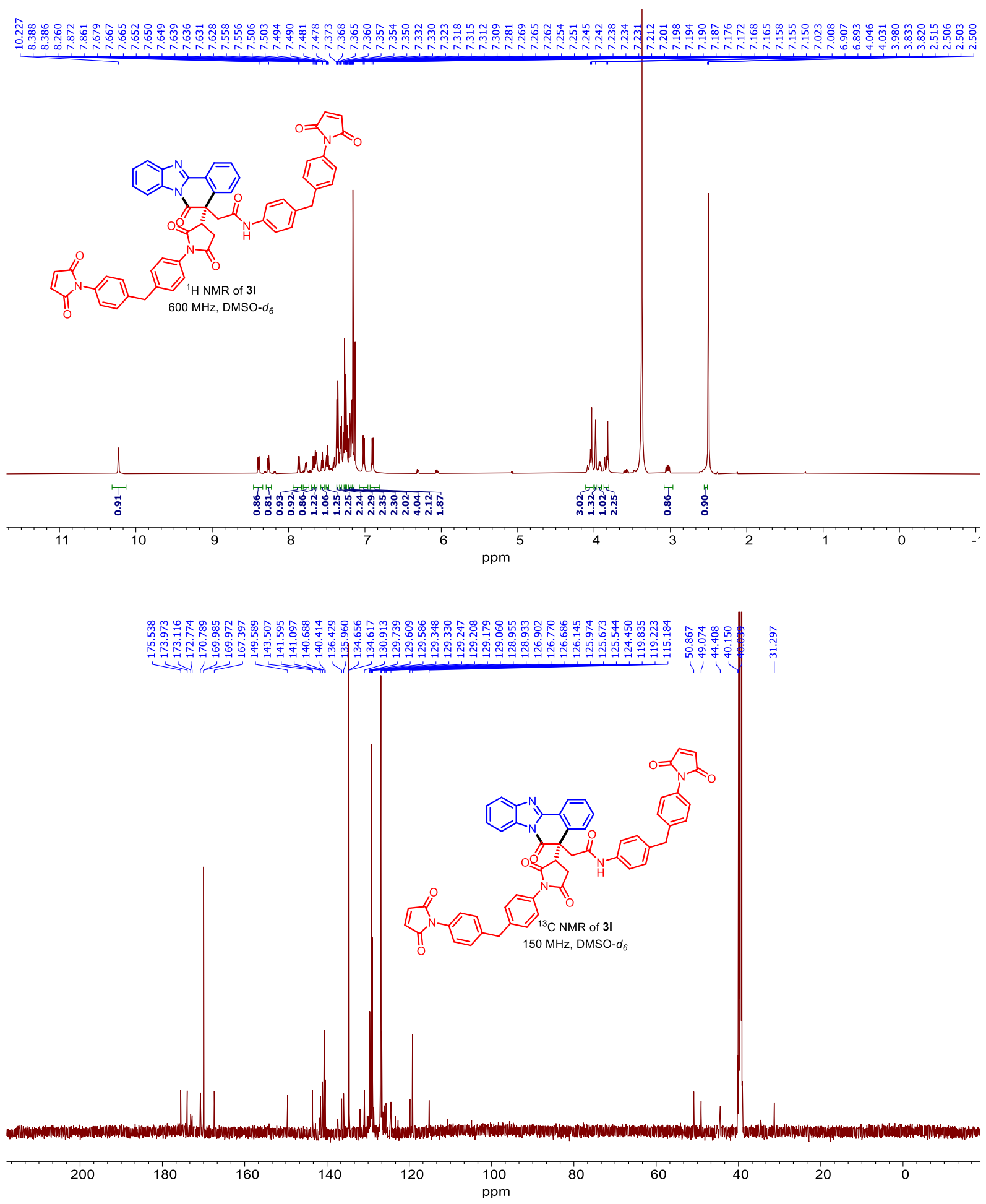


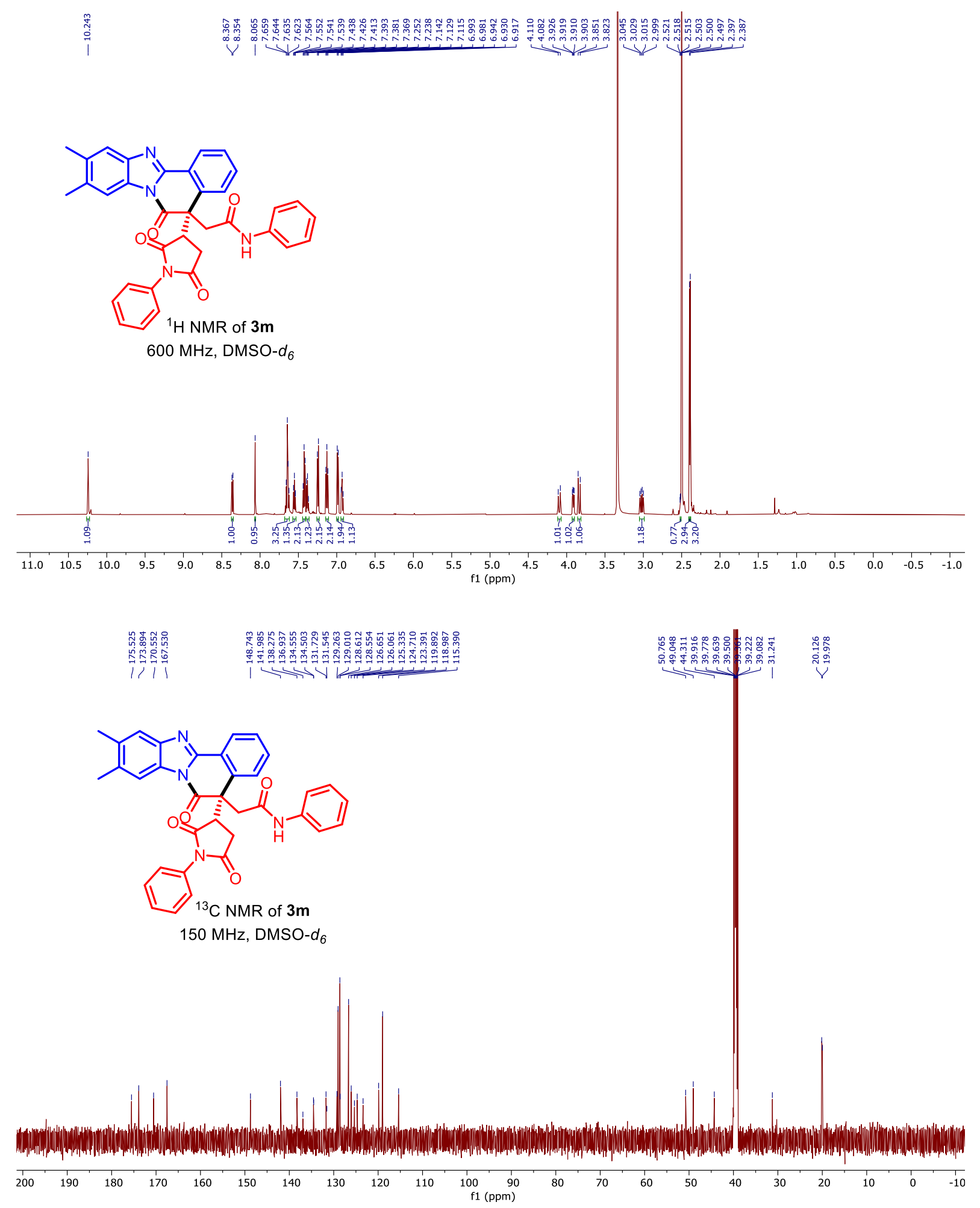



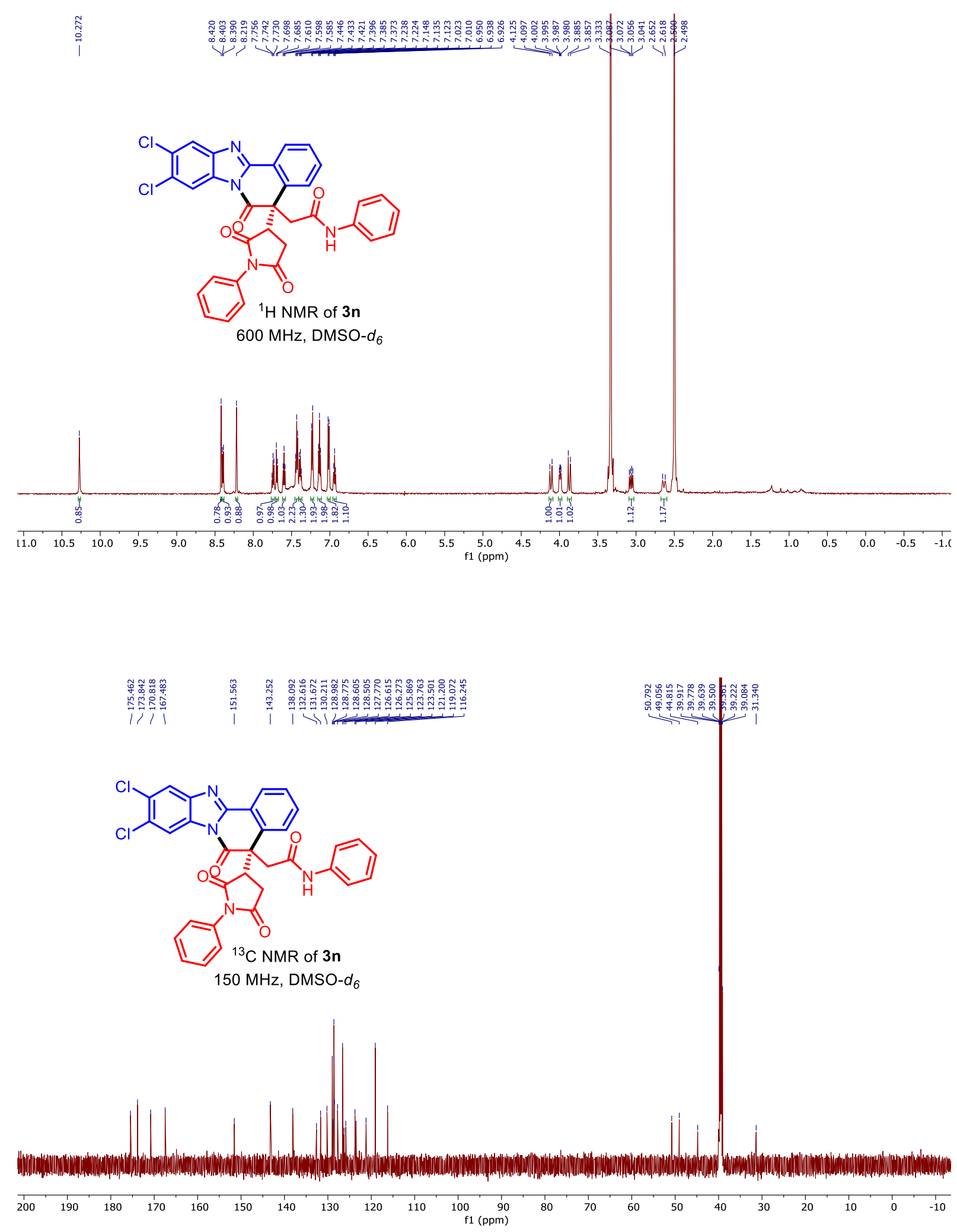


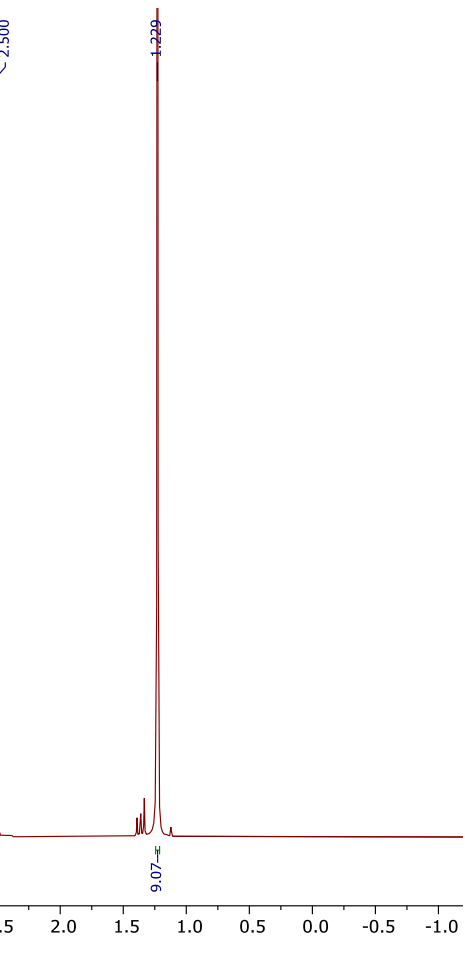

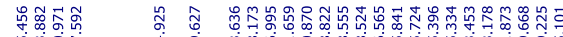

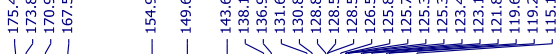

${ }^{1} \mathrm{H}$ NMR of 30

$600 \mathrm{MHz}, \mathrm{DMSO}-d_{6}$
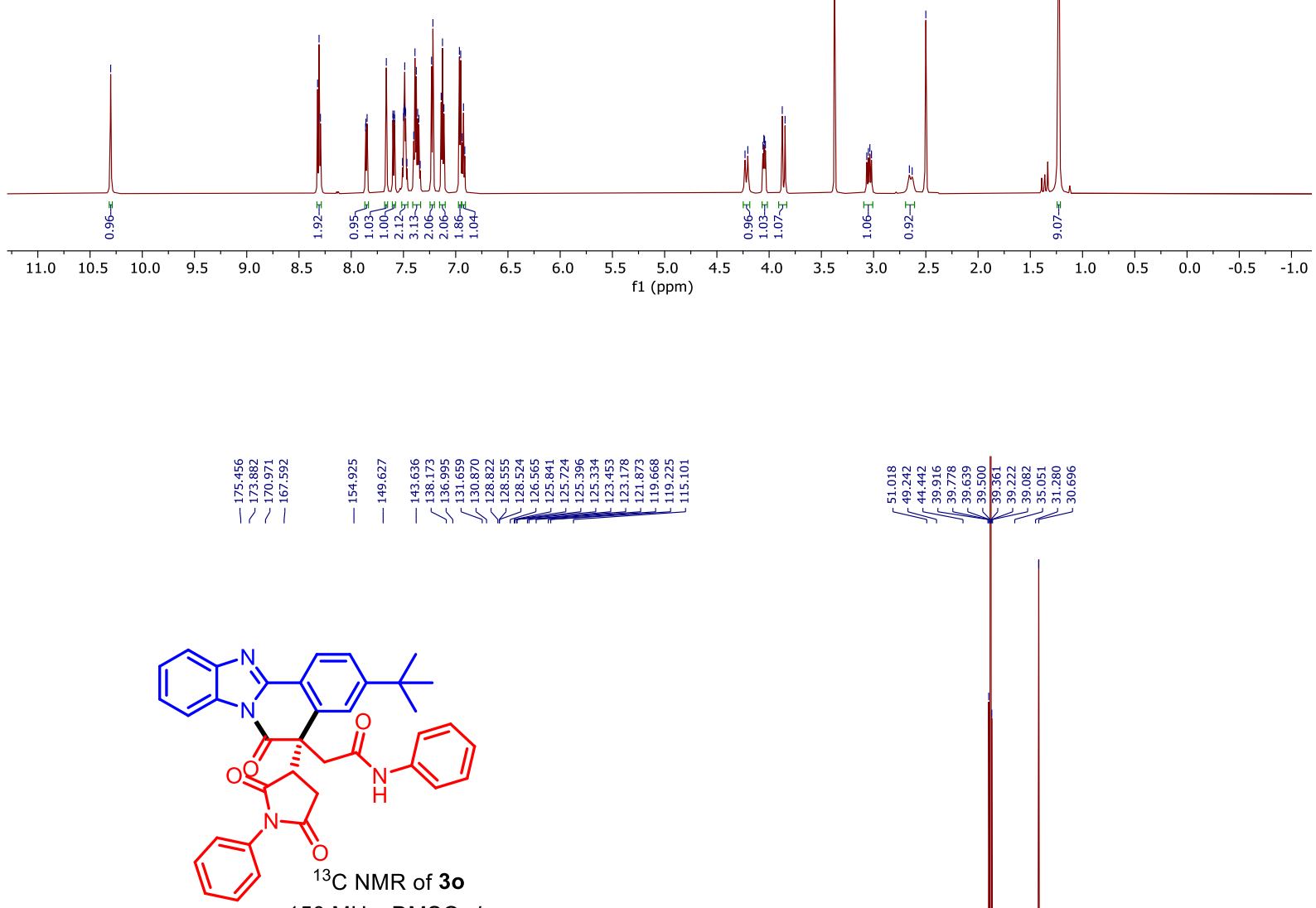

$150 \mathrm{MHz}, \mathrm{DMSO}-d_{6}$

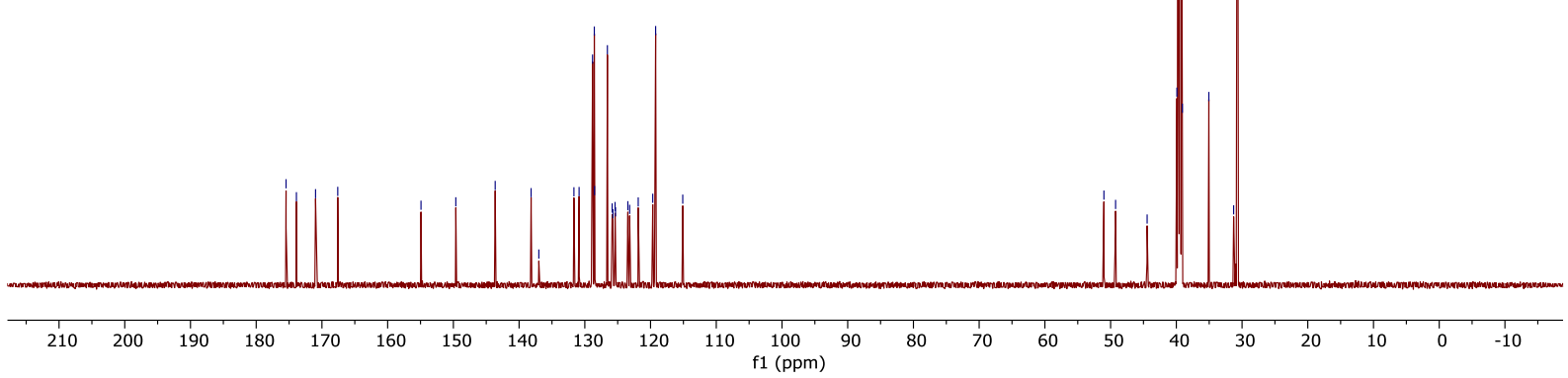



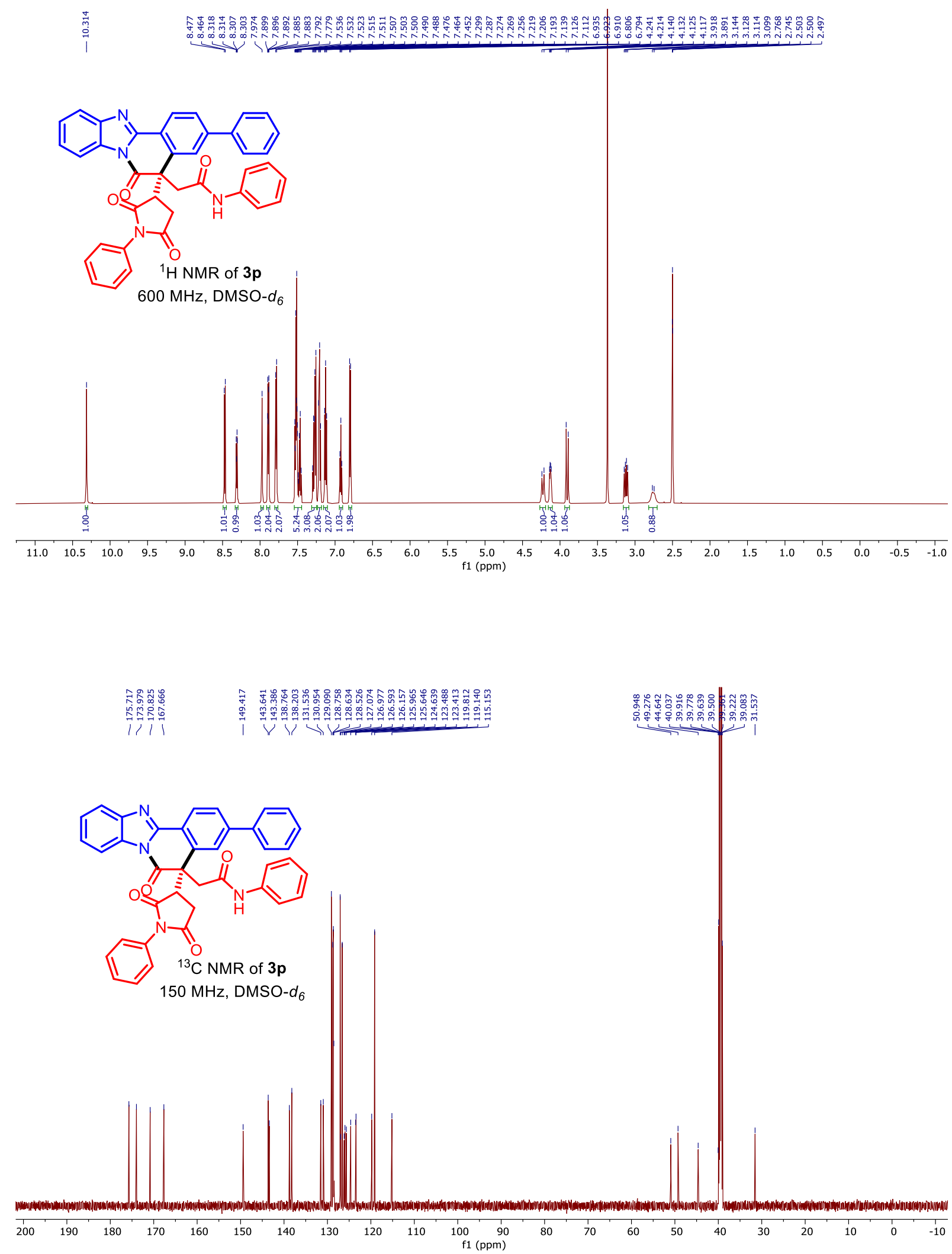

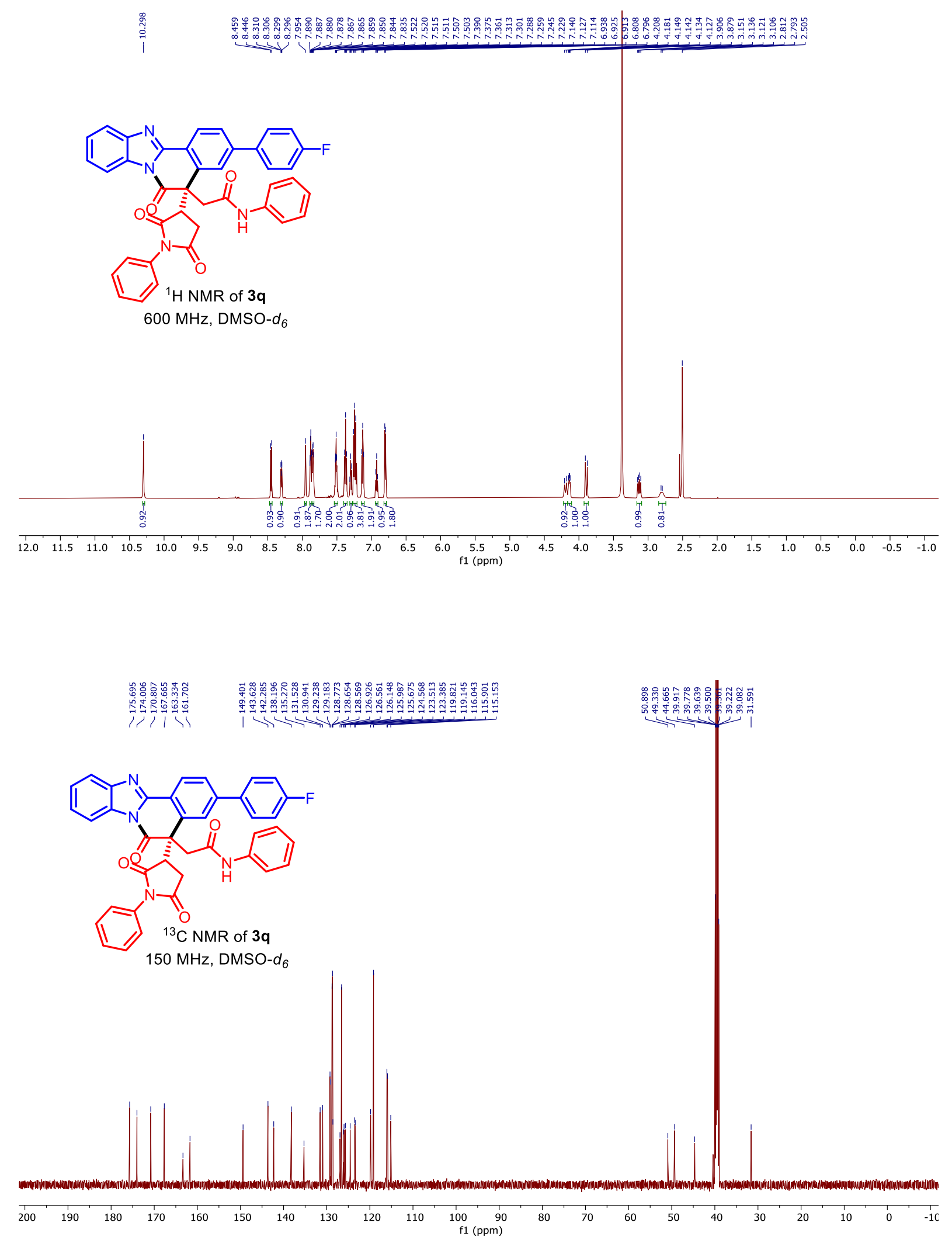

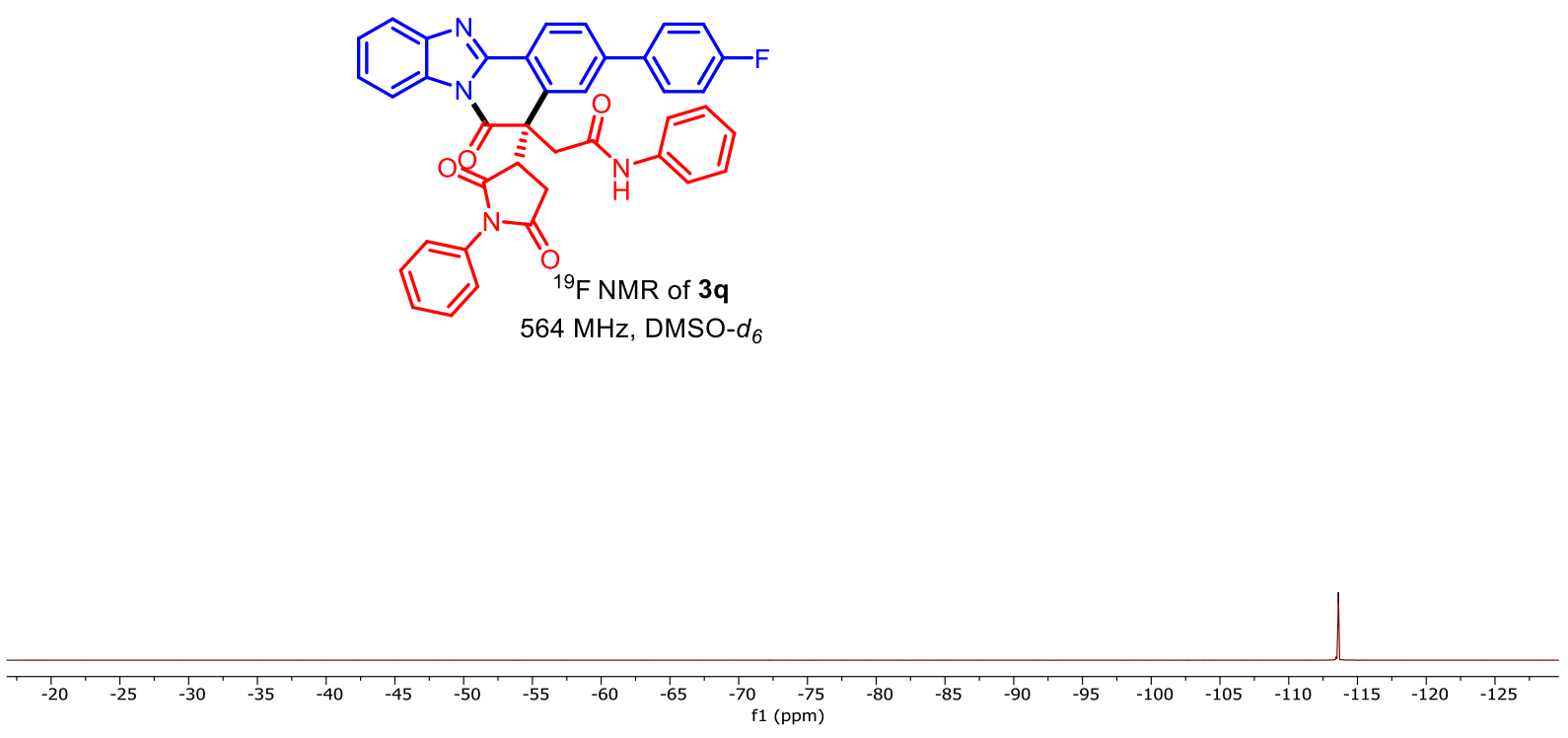

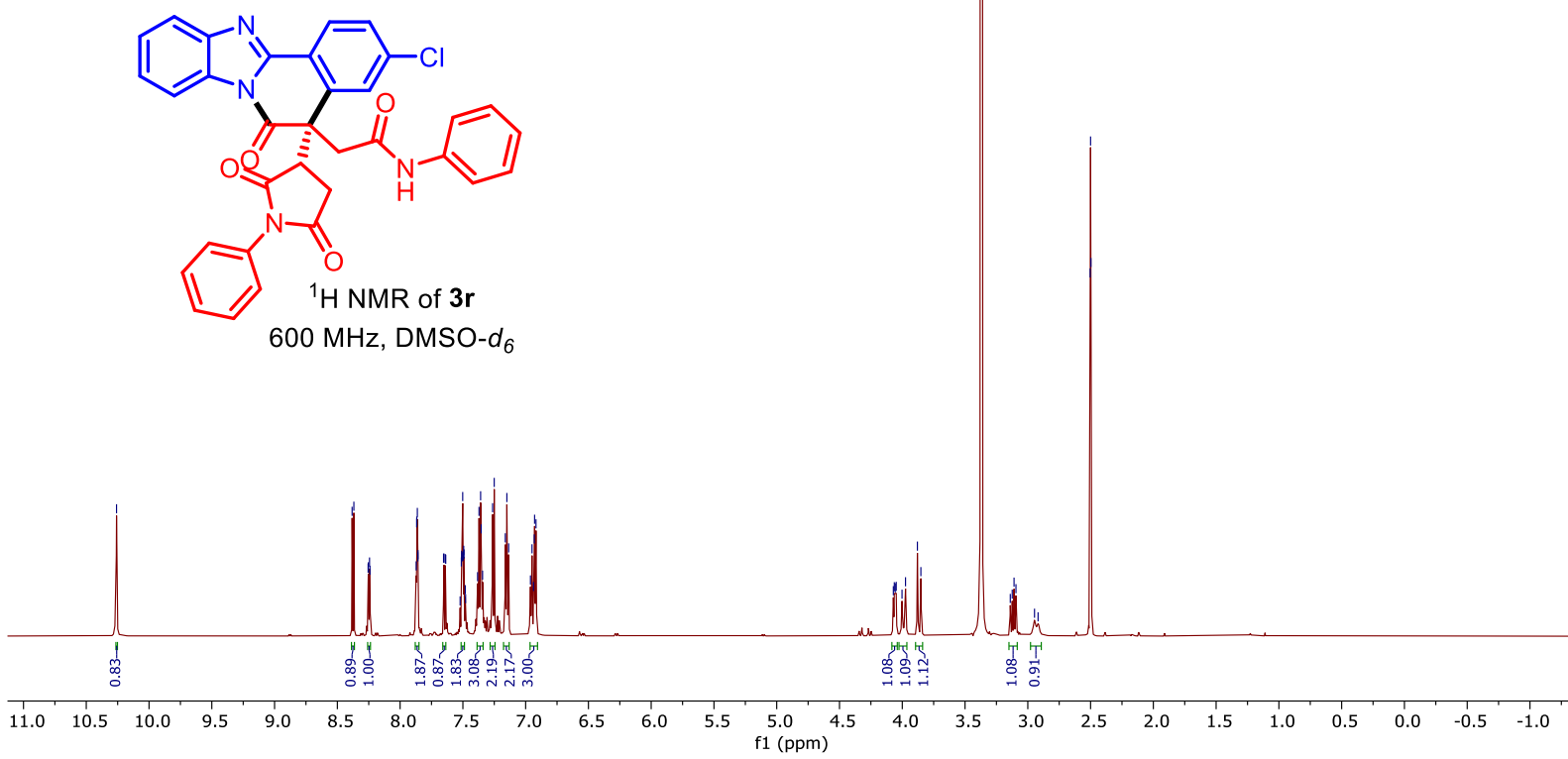

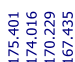

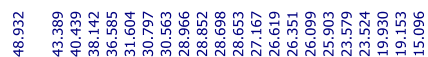

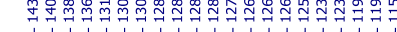

1) 11

$1111<1$

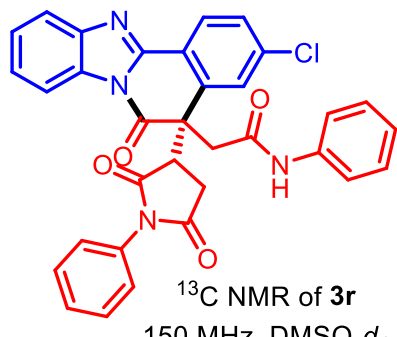

$150 \mathrm{MHz}, \mathrm{DMSO}-d_{6}$

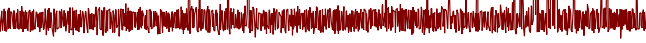

$\begin{array}{rlllllllllll}7200 & 190 & 180 & 170 & 160 & 150 & 140 & 130 & 120 & 110 & 1 & 100 \\ \mathrm{f} 1(\mathrm{ppm})\end{array}$ 

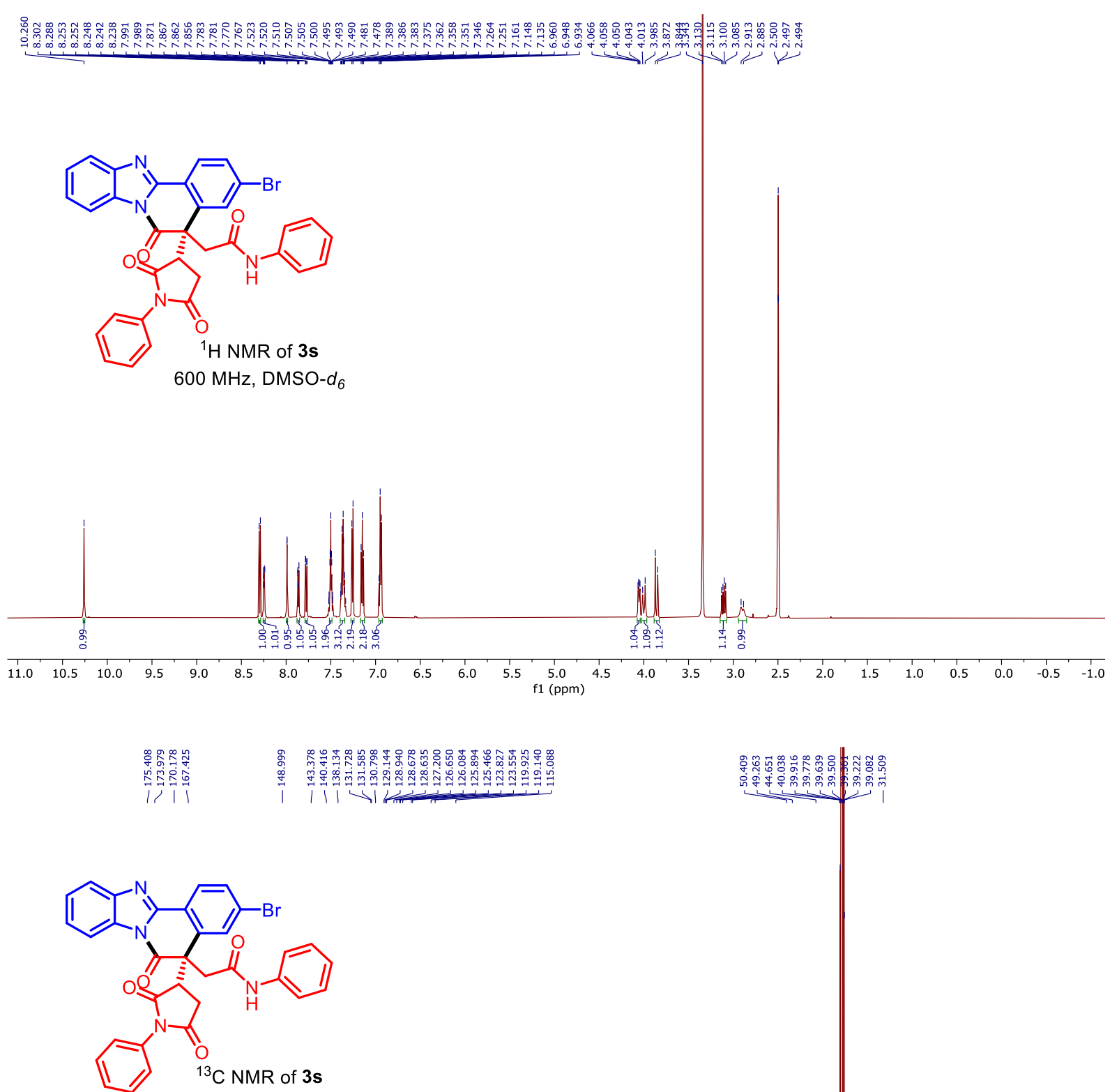

$150 \mathrm{MHz}$, DMSO- $d_{6}$

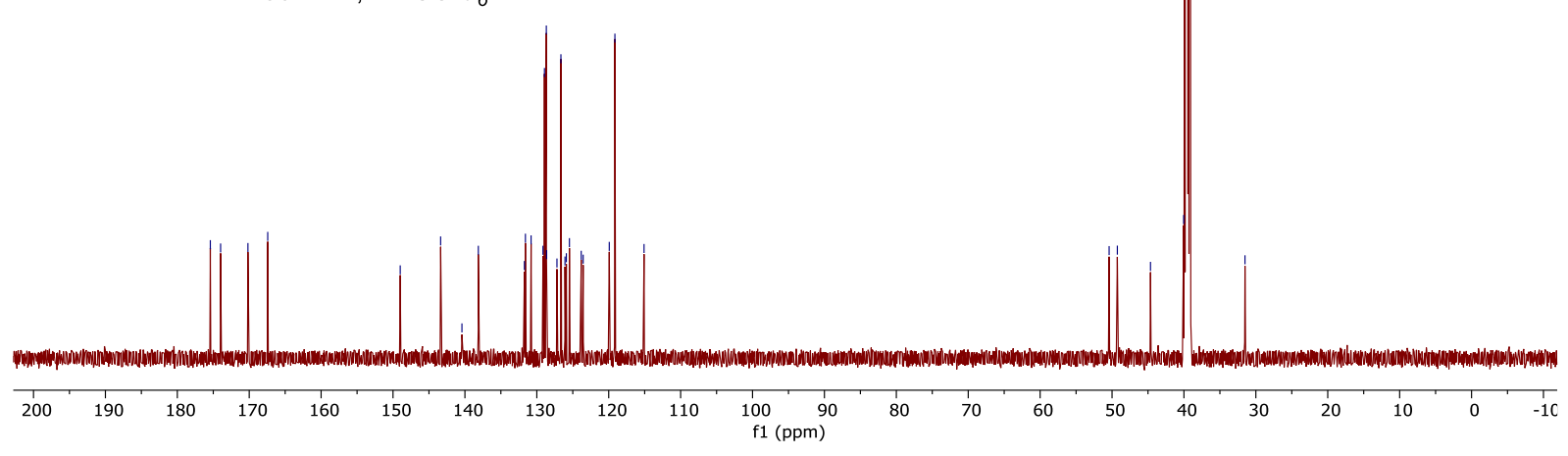




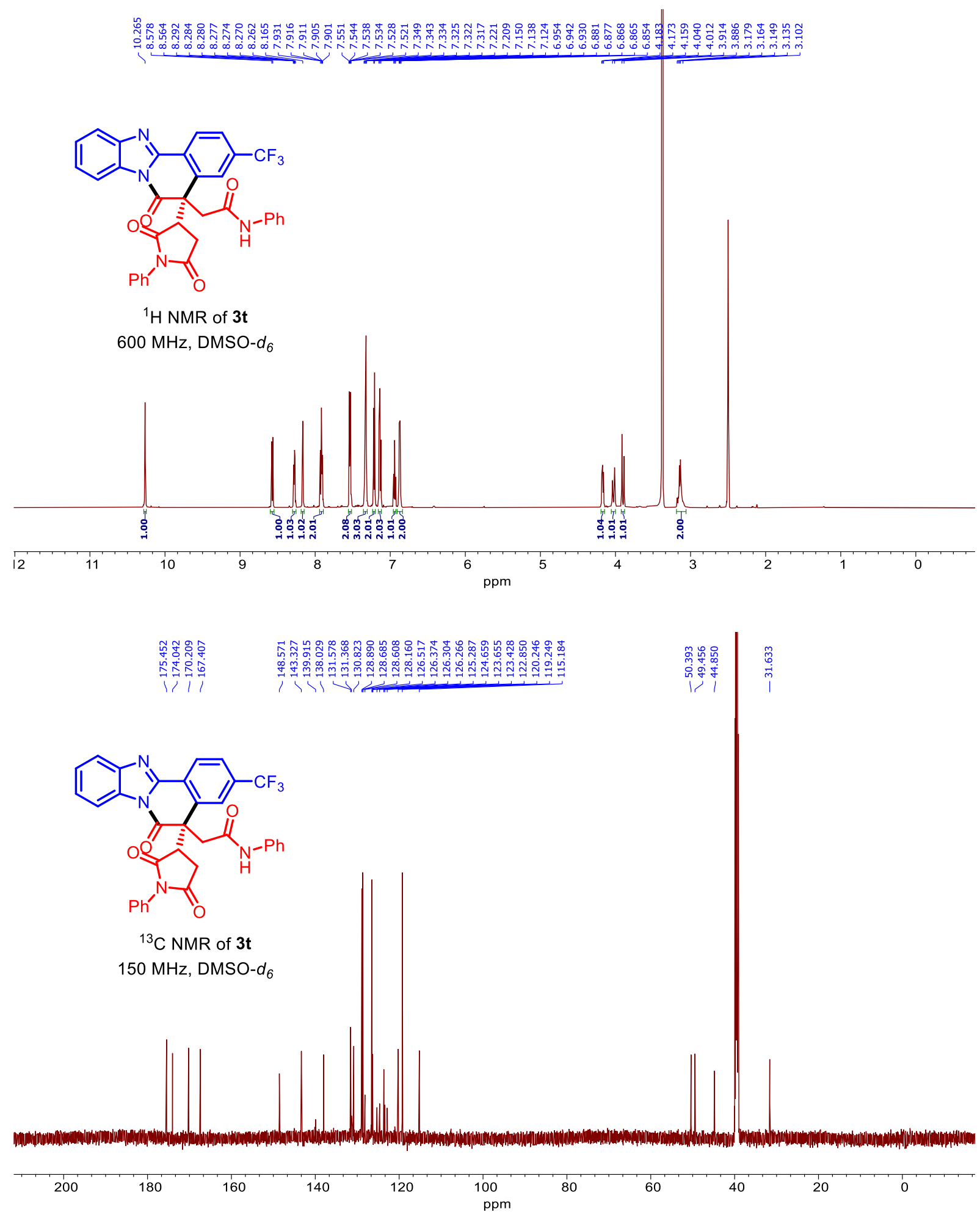




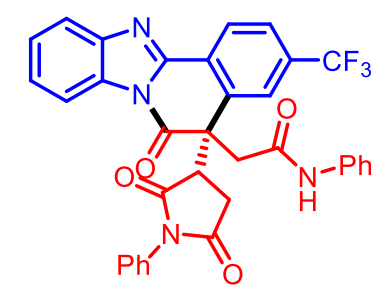

${ }^{19} \mathrm{~F} \mathrm{NMR}$ of $\mathbf{3 t}$

$565 \mathrm{MHz}, \mathrm{DMSO}-d_{6}$

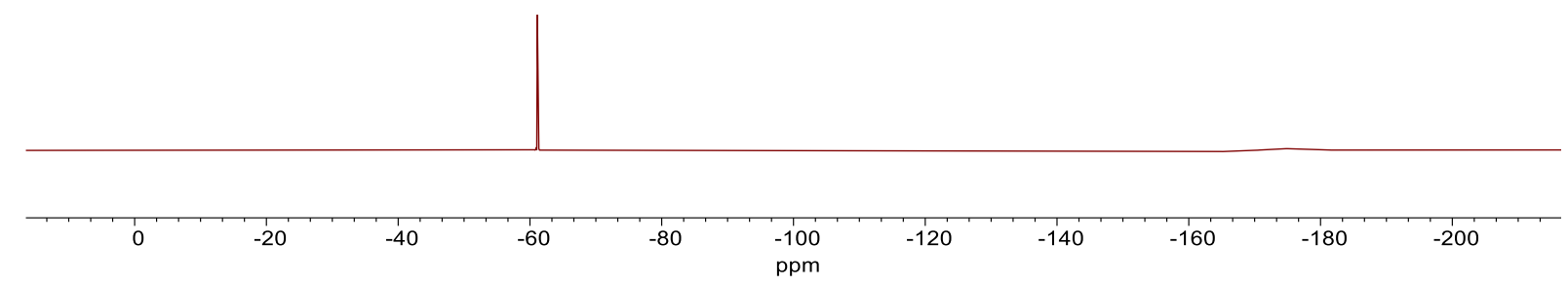



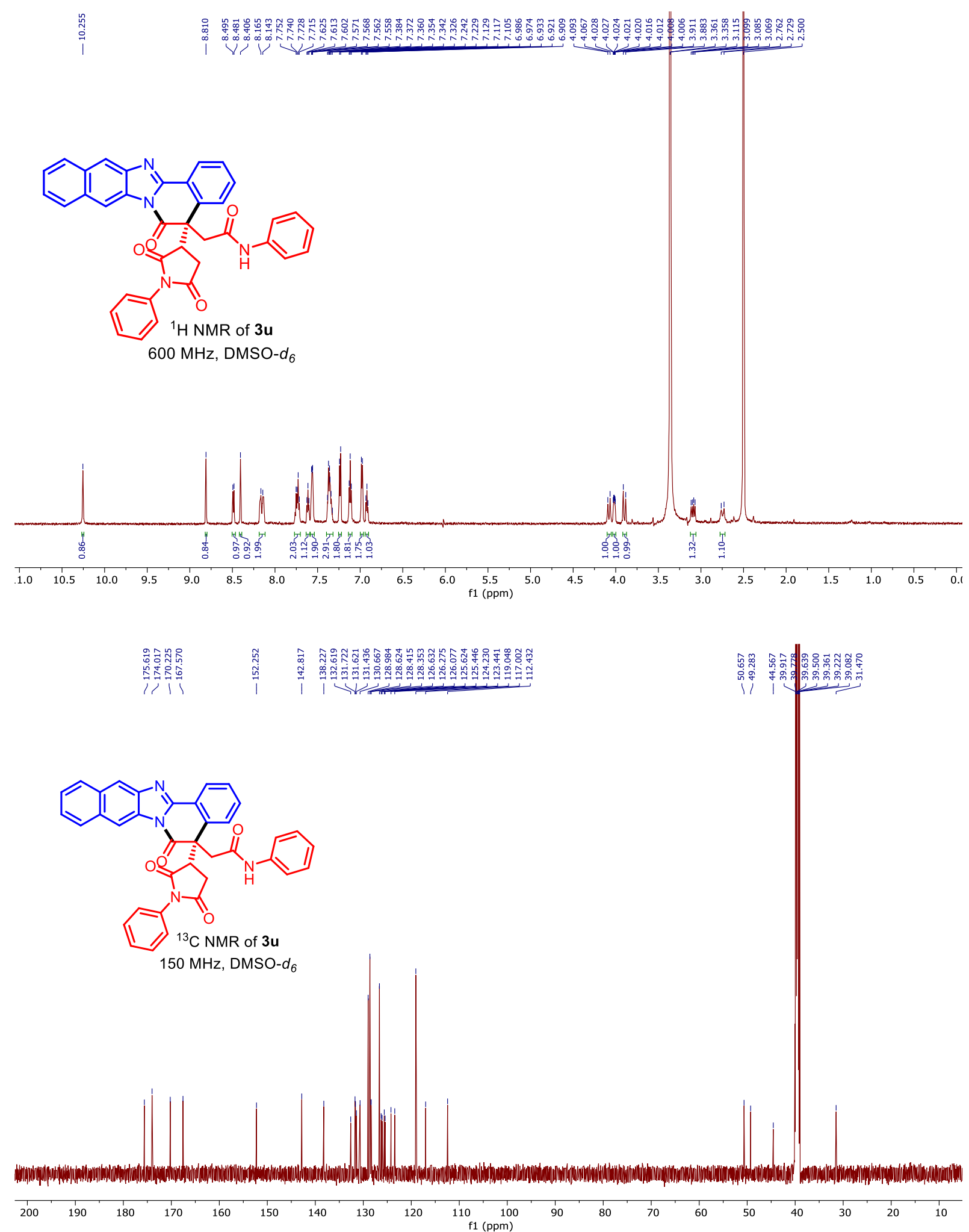

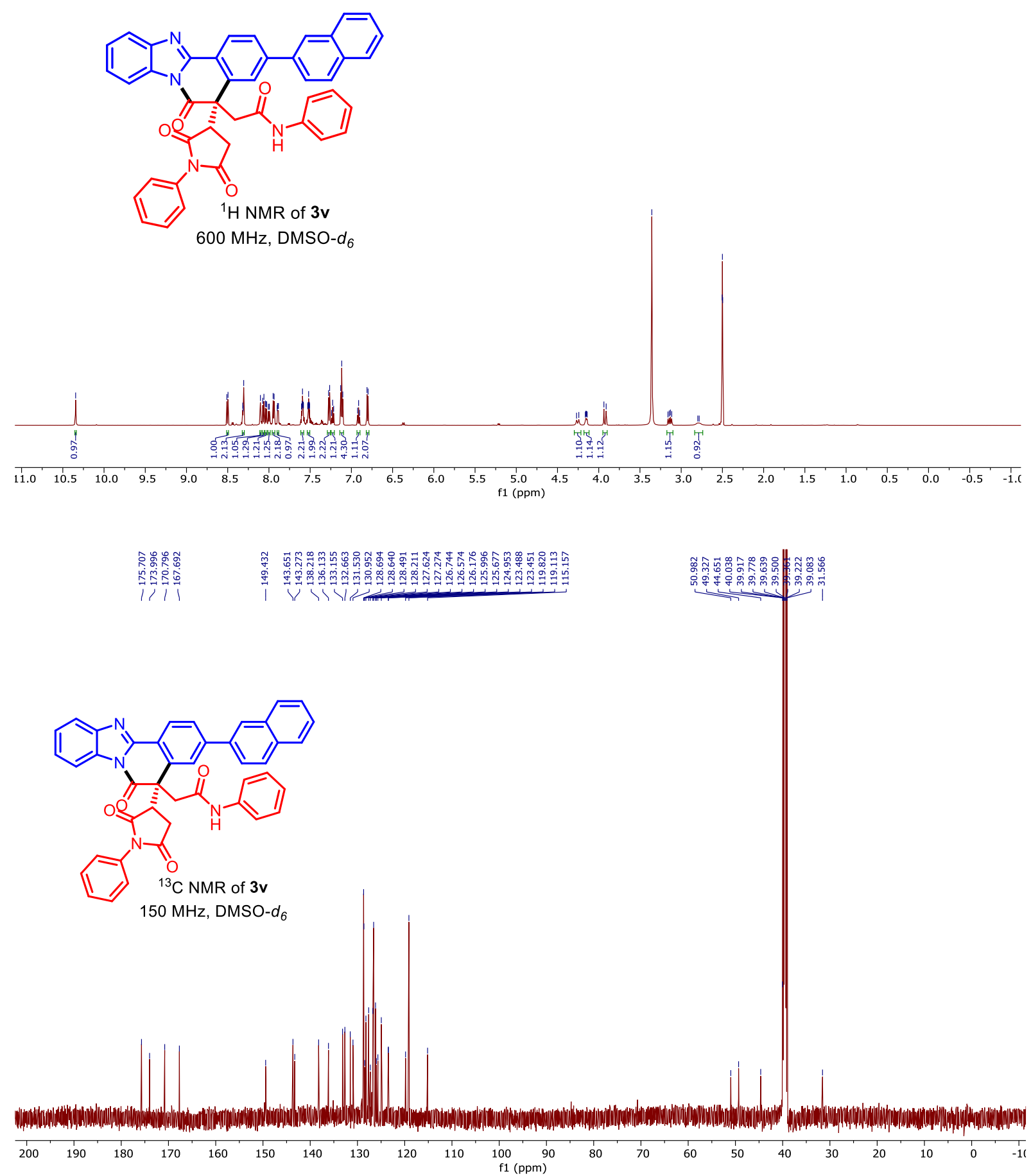


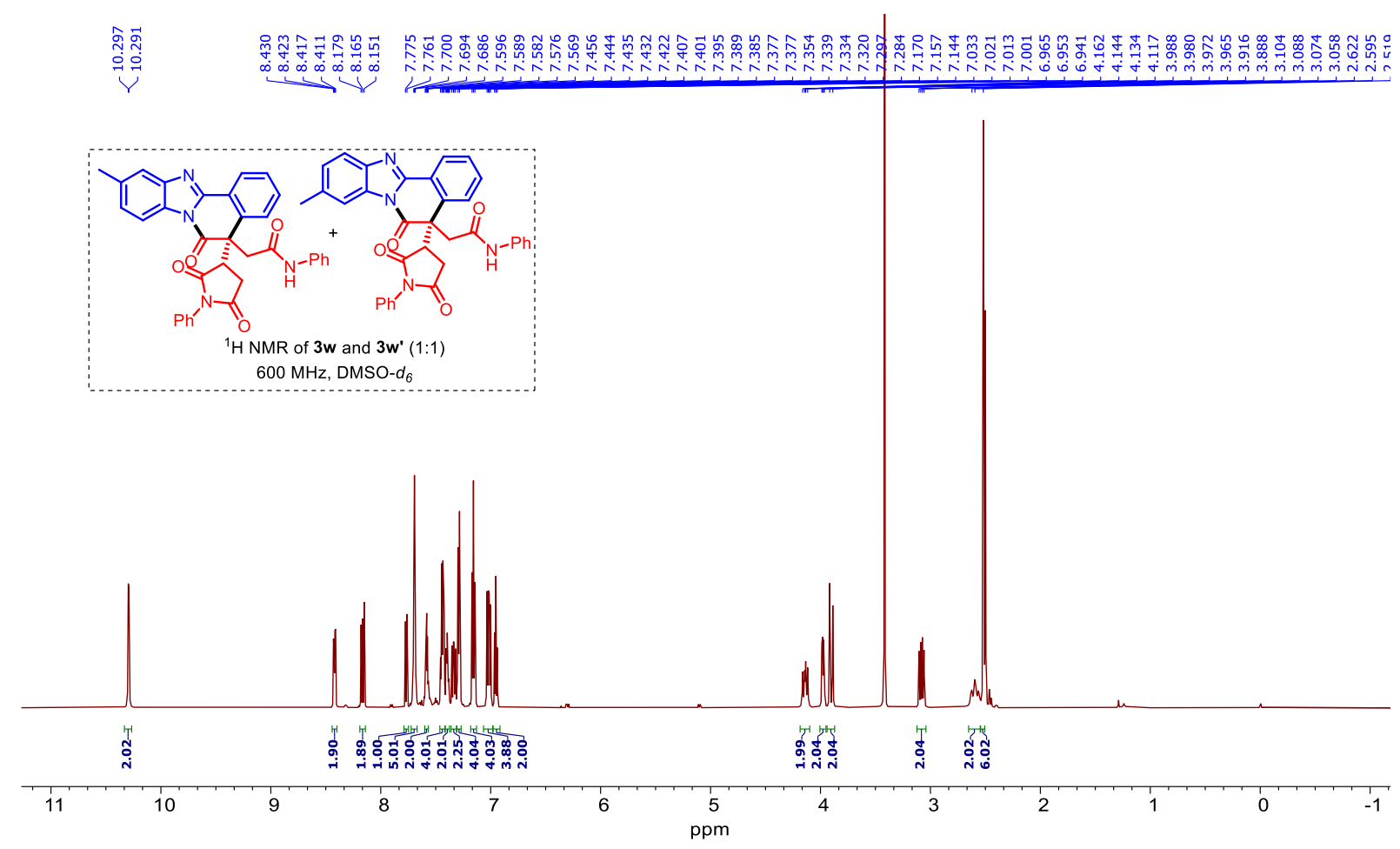

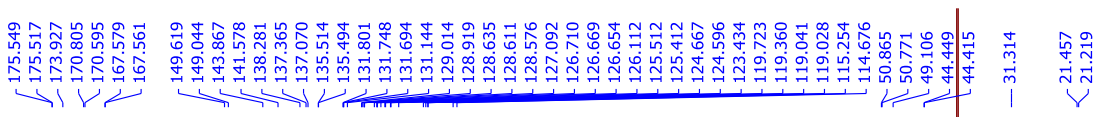

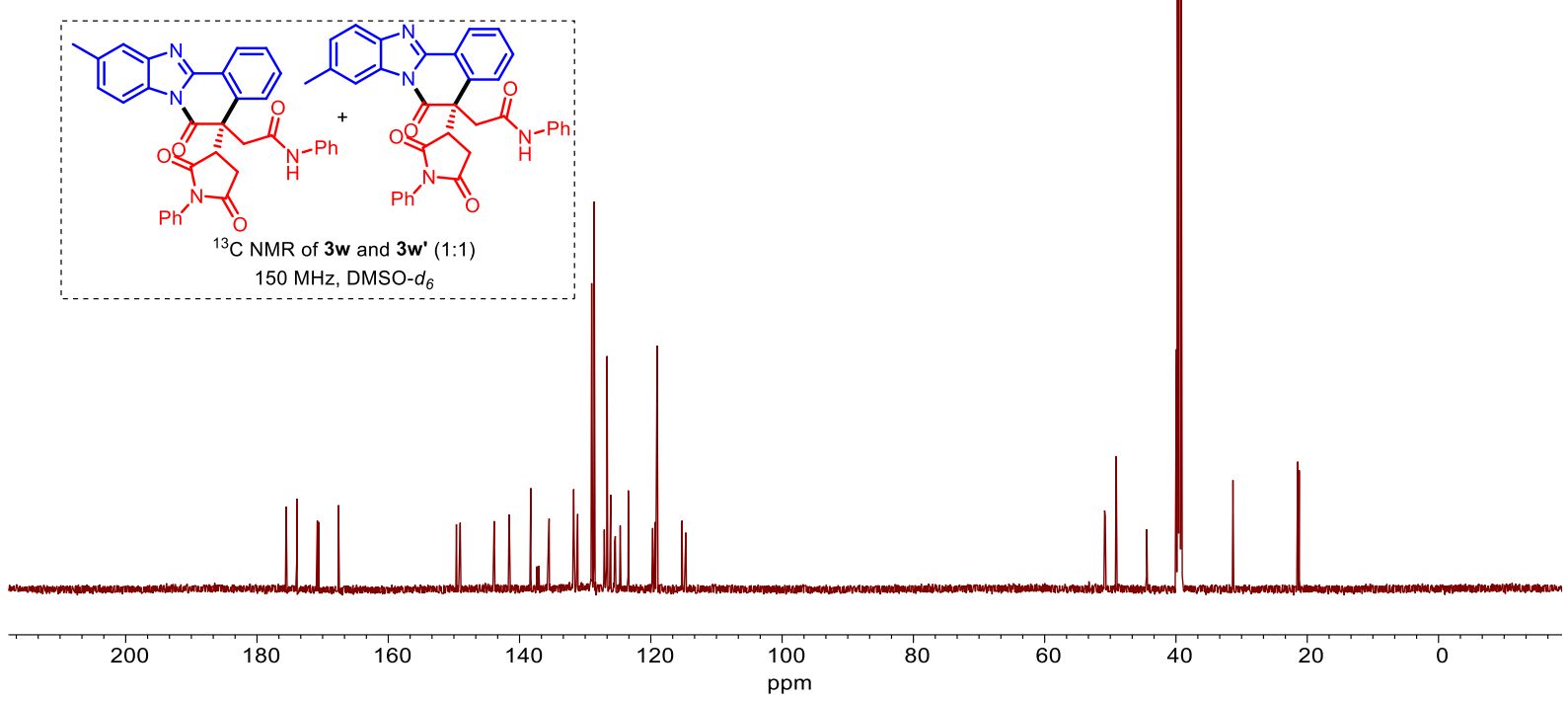




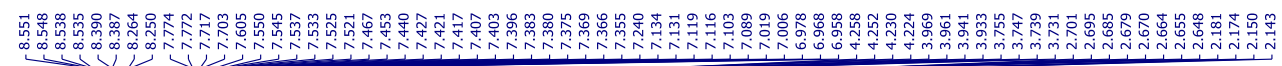
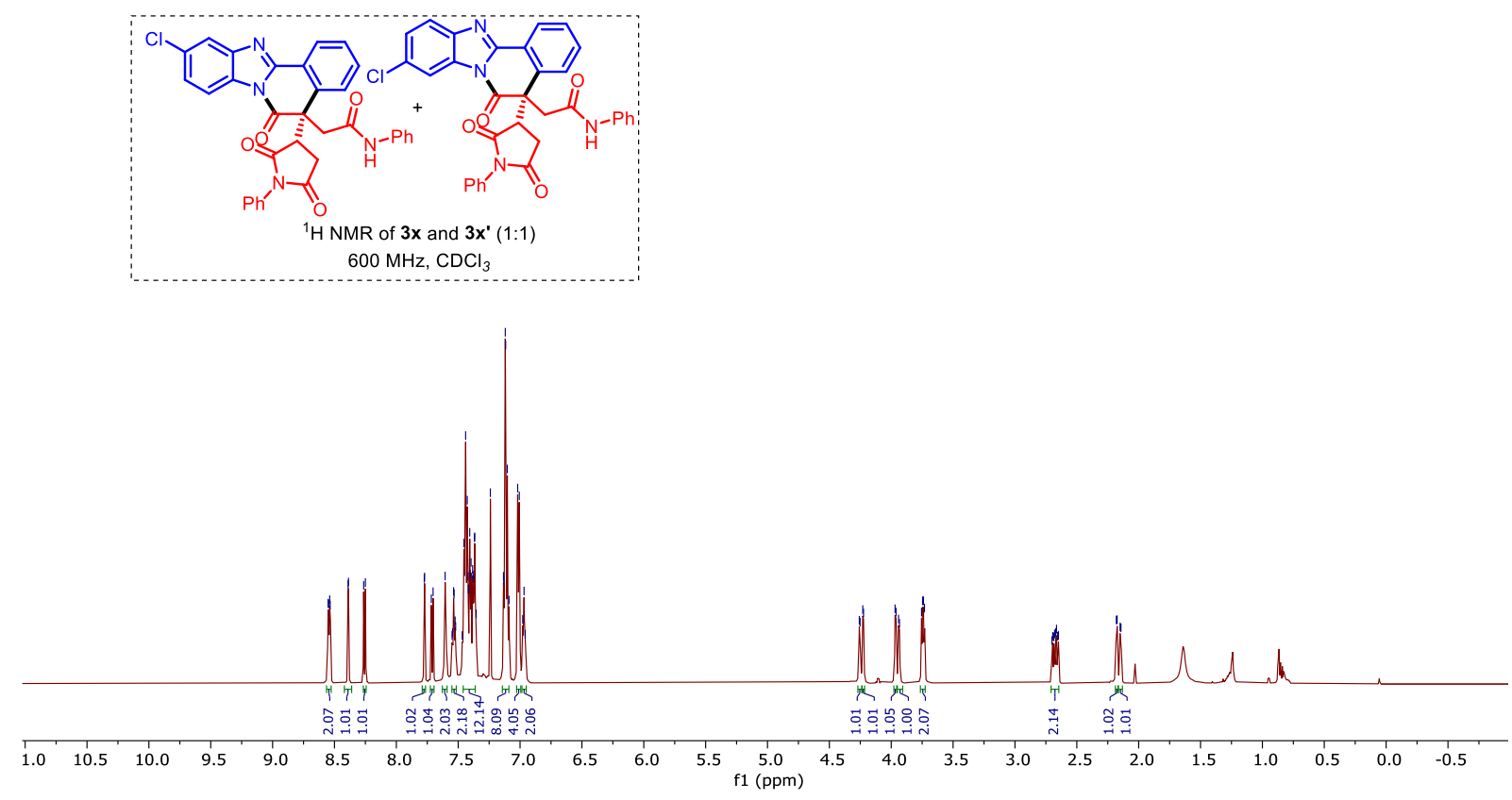

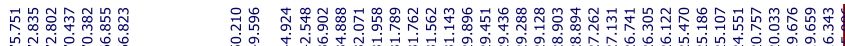

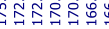

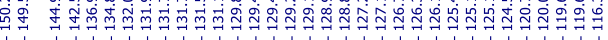

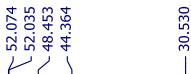
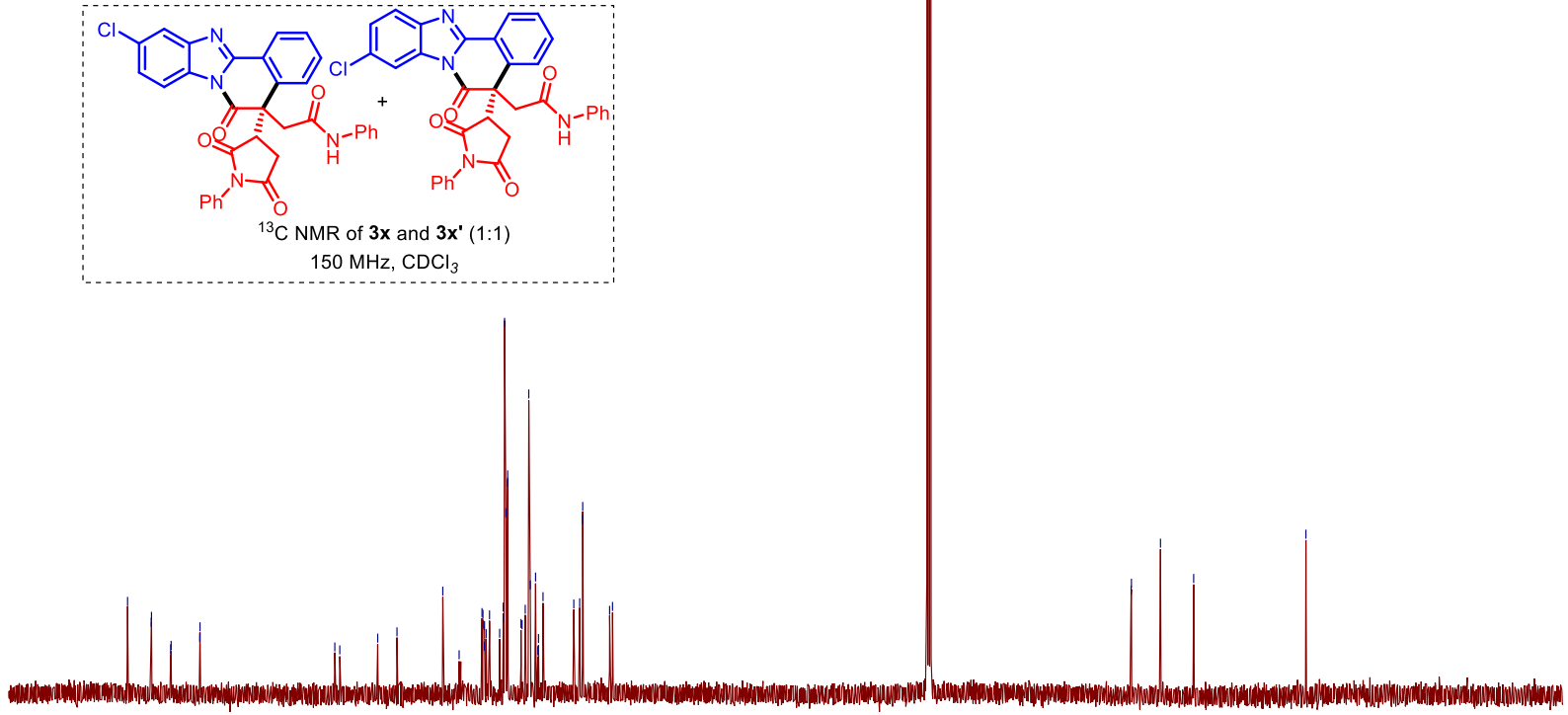

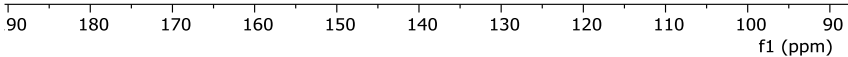

$80 \quad 70 \quad 60$

$\begin{array}{llllll}1 & 1 & 1 & 1 & 1 & 1 \\ 50 & 40 & 30 & 20 & 10 & 0\end{array}$ 


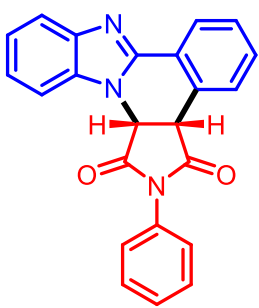

${ }^{1} \mathrm{H}$ NMR of $4 a$

$600 \mathrm{MHz}, \mathrm{CDCl}_{3}$
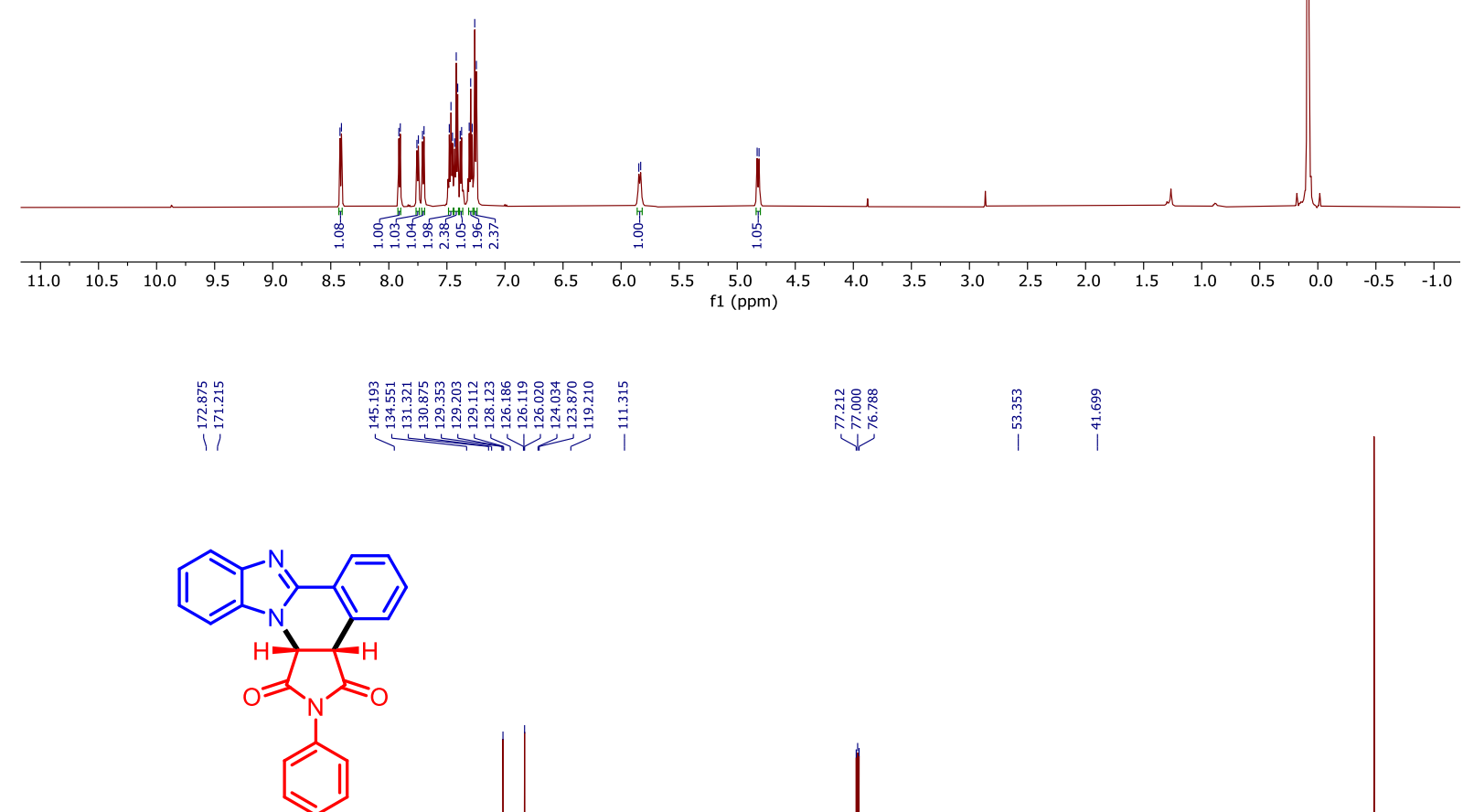

${ }^{13} \mathrm{C}$ NMR of $4 \mathrm{a}$

$150 \mathrm{MHz}, \mathrm{CDCl}_{3}$

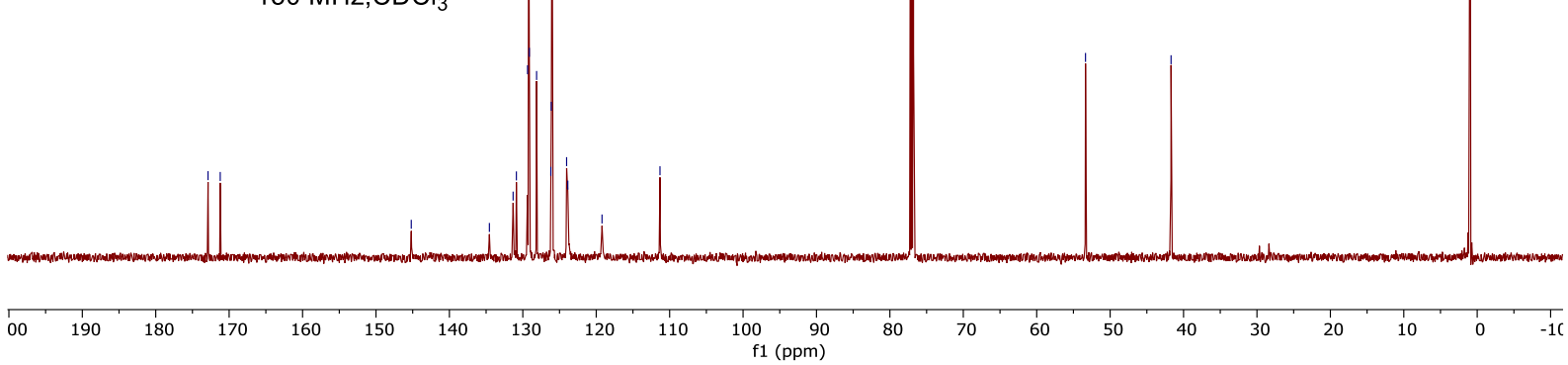



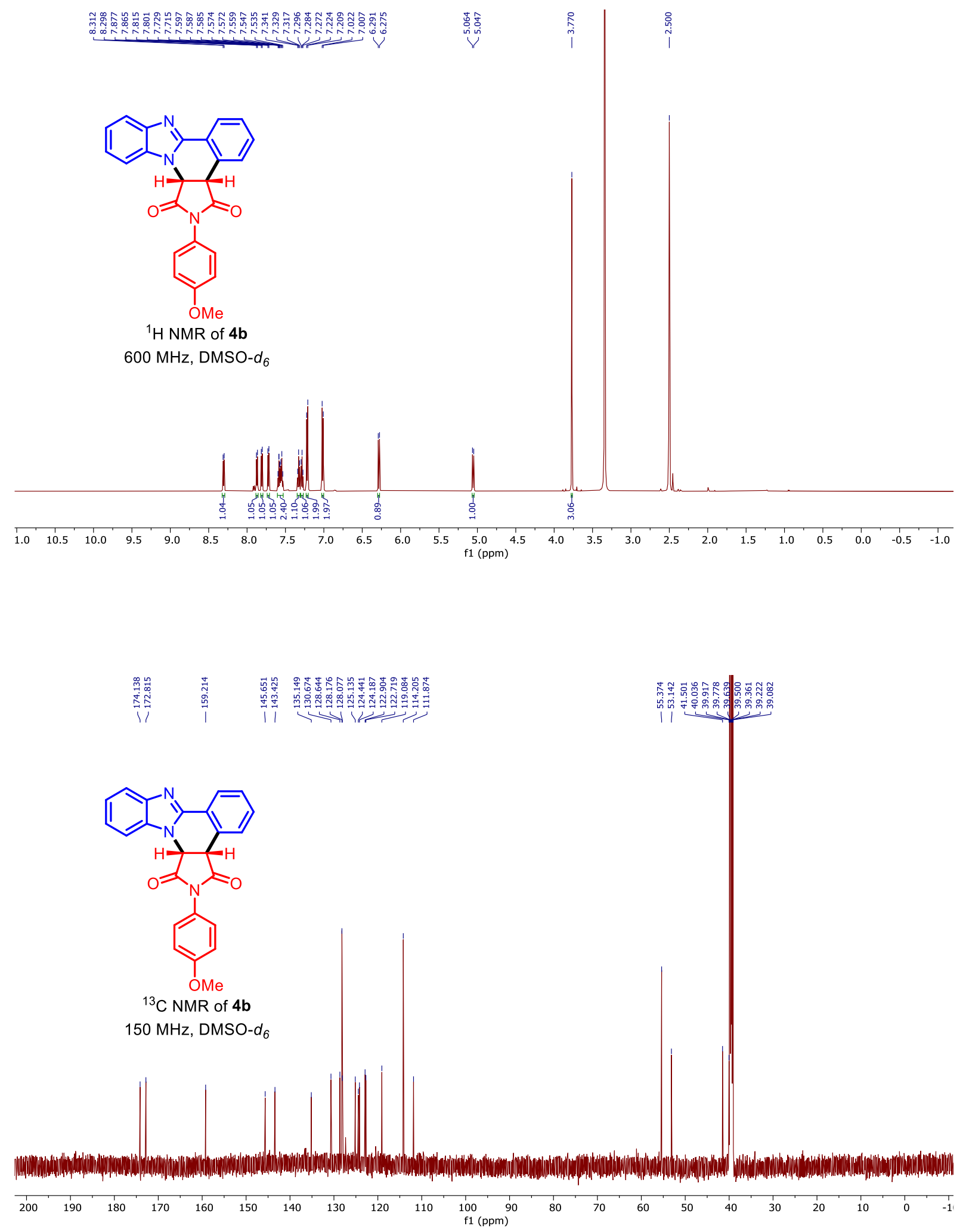

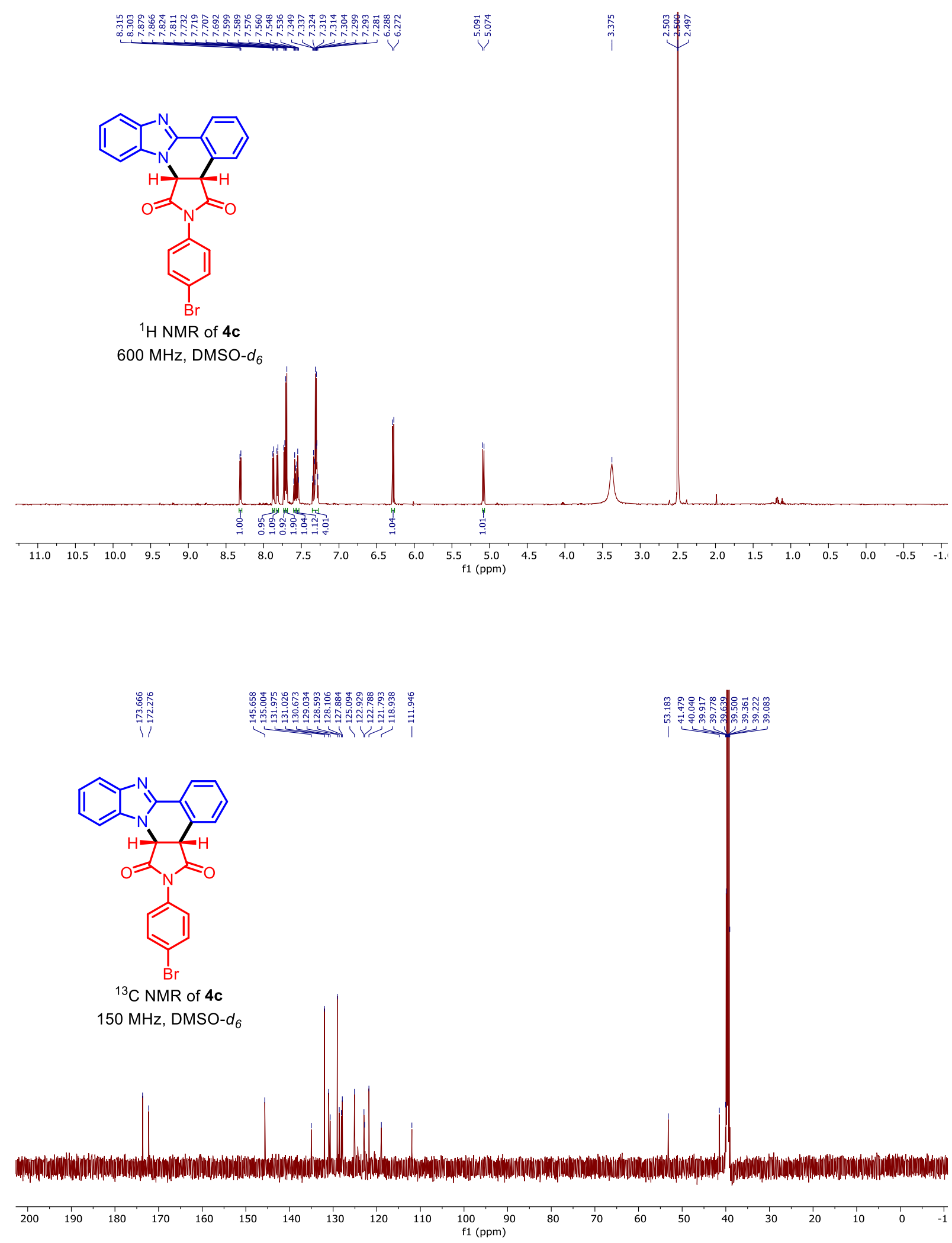


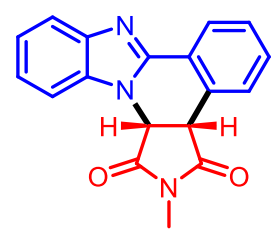

${ }^{1} \mathrm{H}$ NMR of $4 d$

$600 \mathrm{MHz}, \mathrm{CDCl}_{3}$

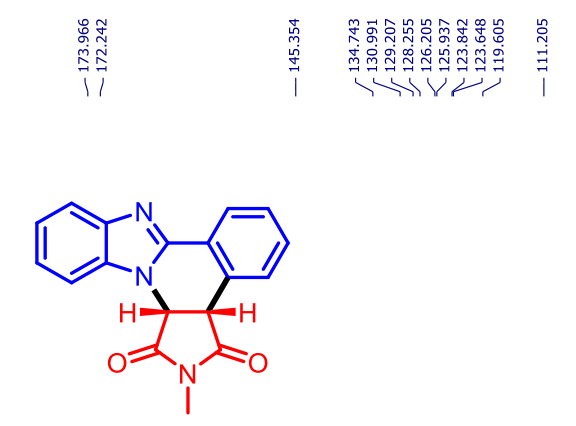

${ }^{13} \mathrm{C}$ NMR of $4 \mathbf{d}$

$150 \mathrm{MHz}, \mathrm{CDCl}_{3}$

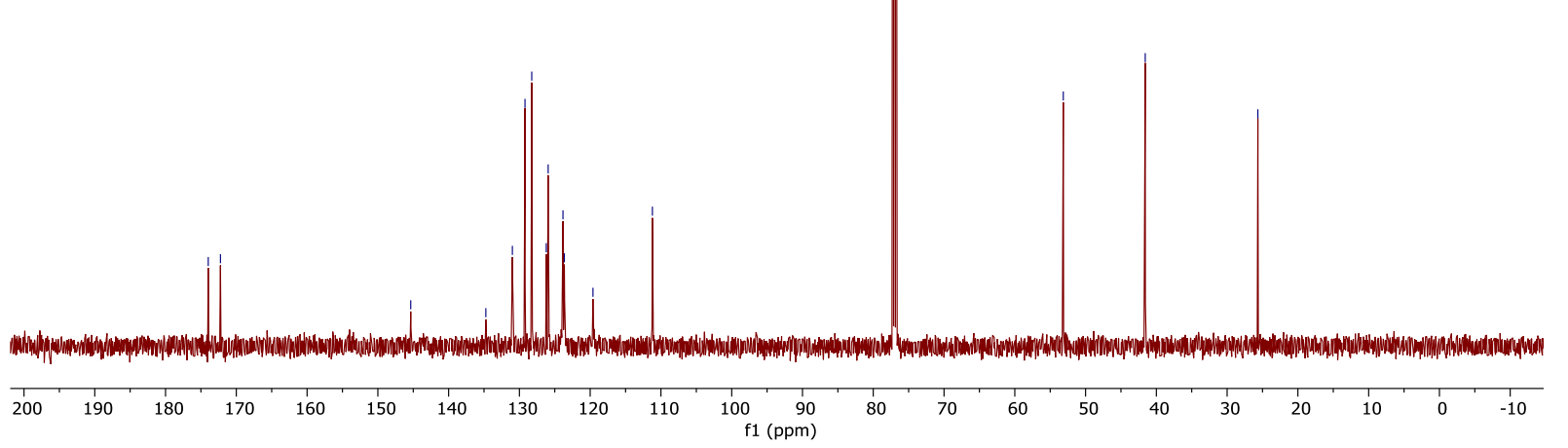




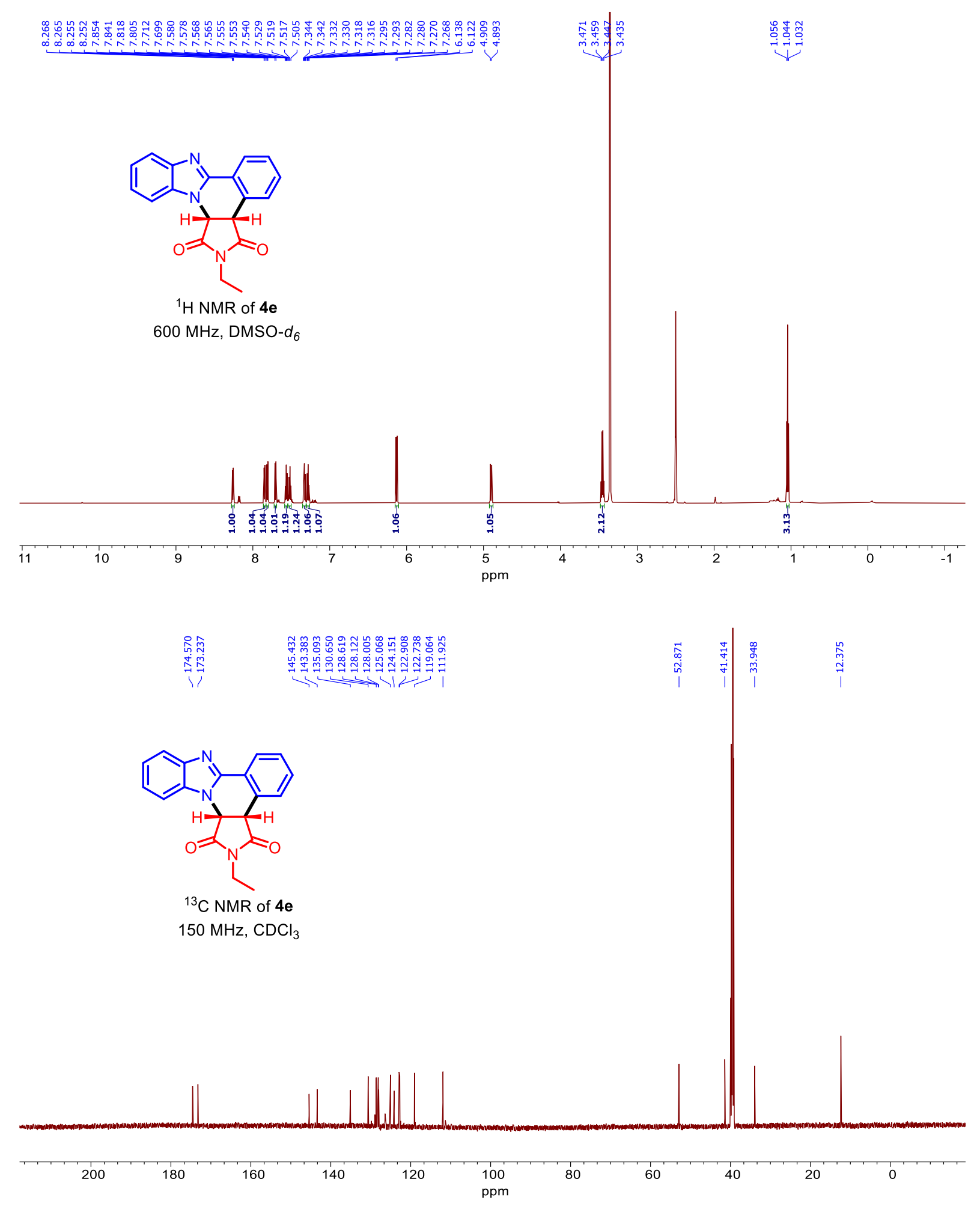




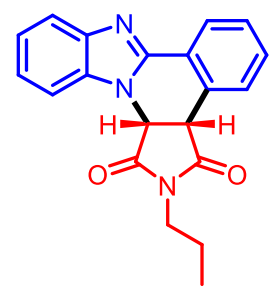

${ }^{1} \mathrm{H}$ NMR of $\mathbf{4} f$

$600 \mathrm{MHz}, \mathrm{CDCl}_{3}$
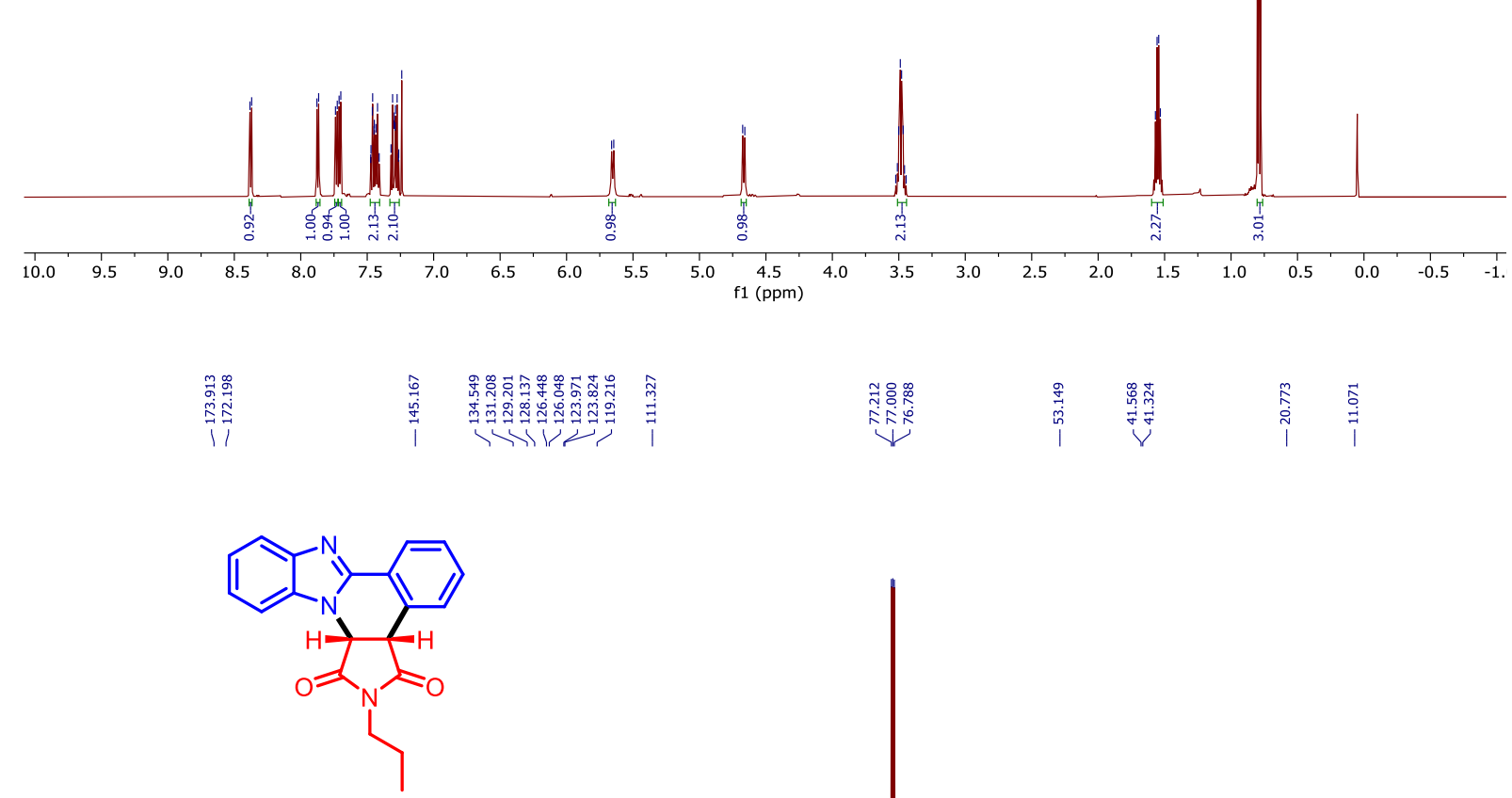

${ }^{13} \mathrm{C}$ NMR of $4 \mathrm{f}$

$150 \mathrm{MHz}, \mathrm{CDCl}_{3}$

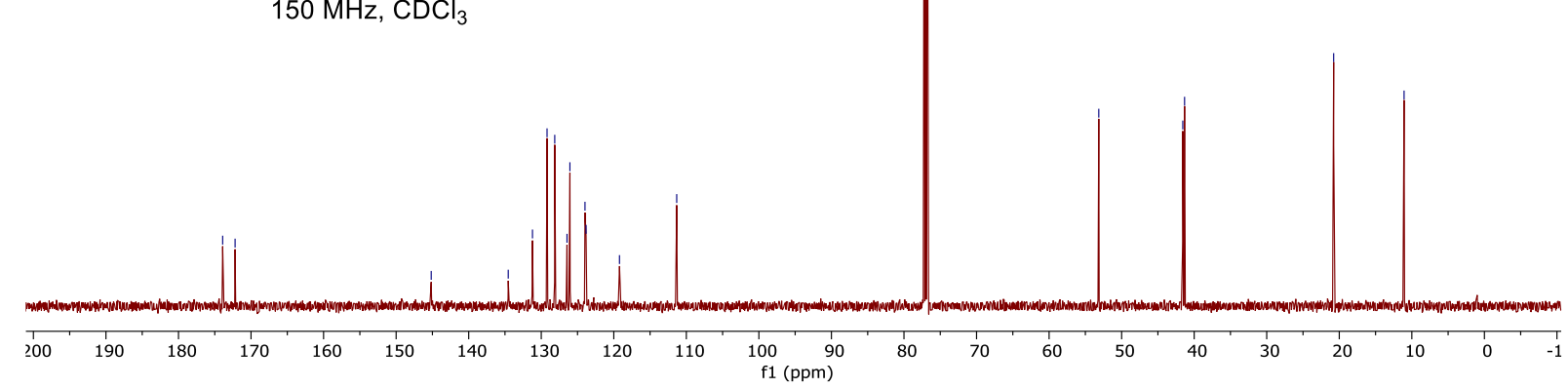




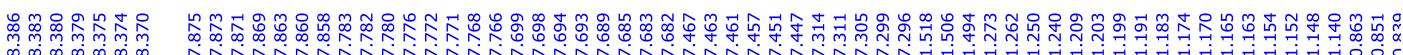
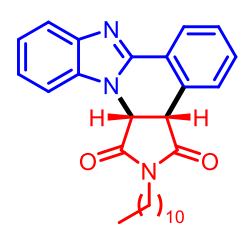

${ }^{1} \mathrm{H}$ NMR of $4 \mathrm{~g}$

$600 \mathrm{MHz}^{\mathrm{CDCl}_{3}}$

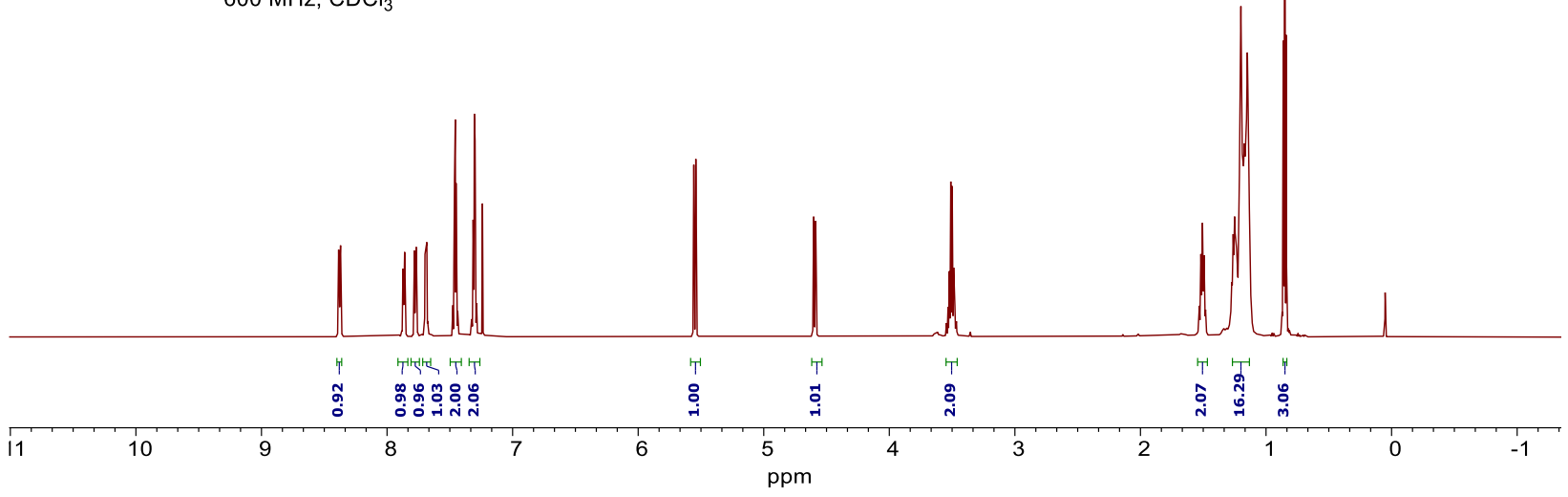

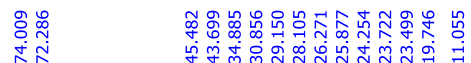

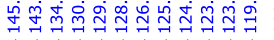

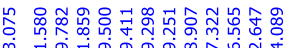

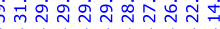

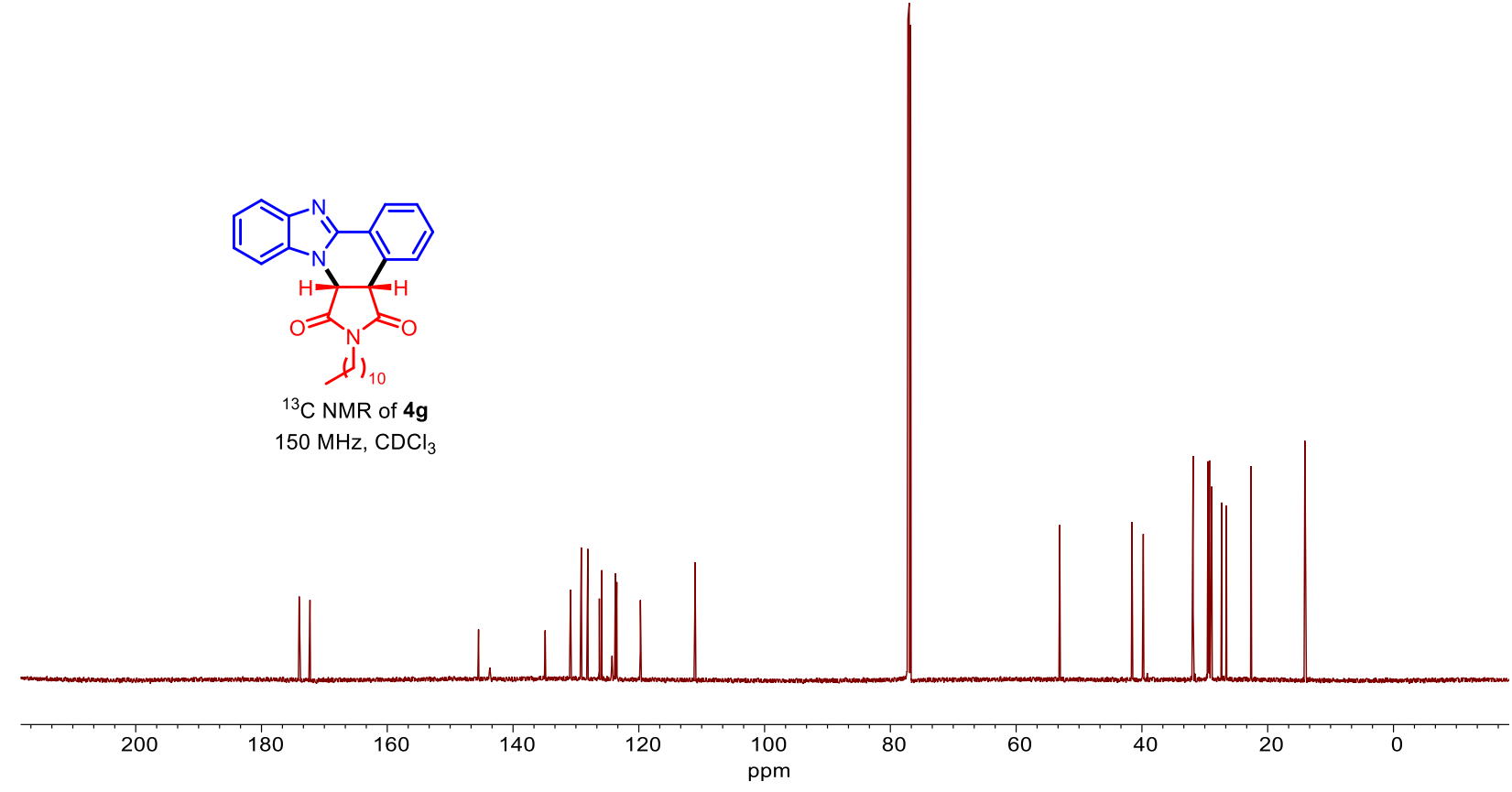



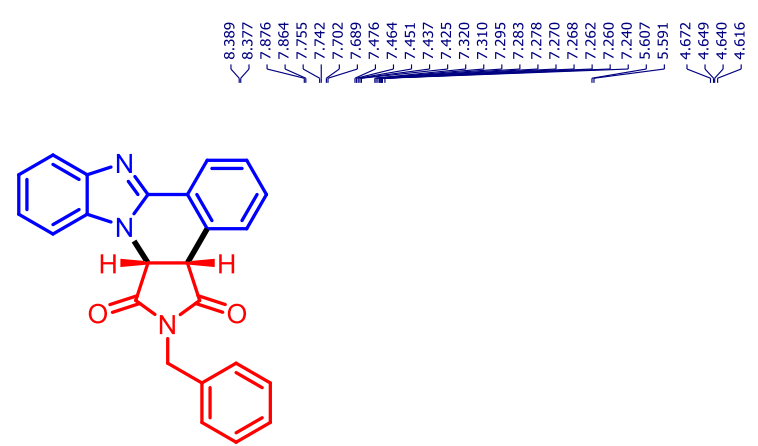

${ }^{1} \mathrm{H}$ NMR of $4 \mathrm{~h}$ $600 \mathrm{MHz}, \mathrm{CDCl}_{3}$
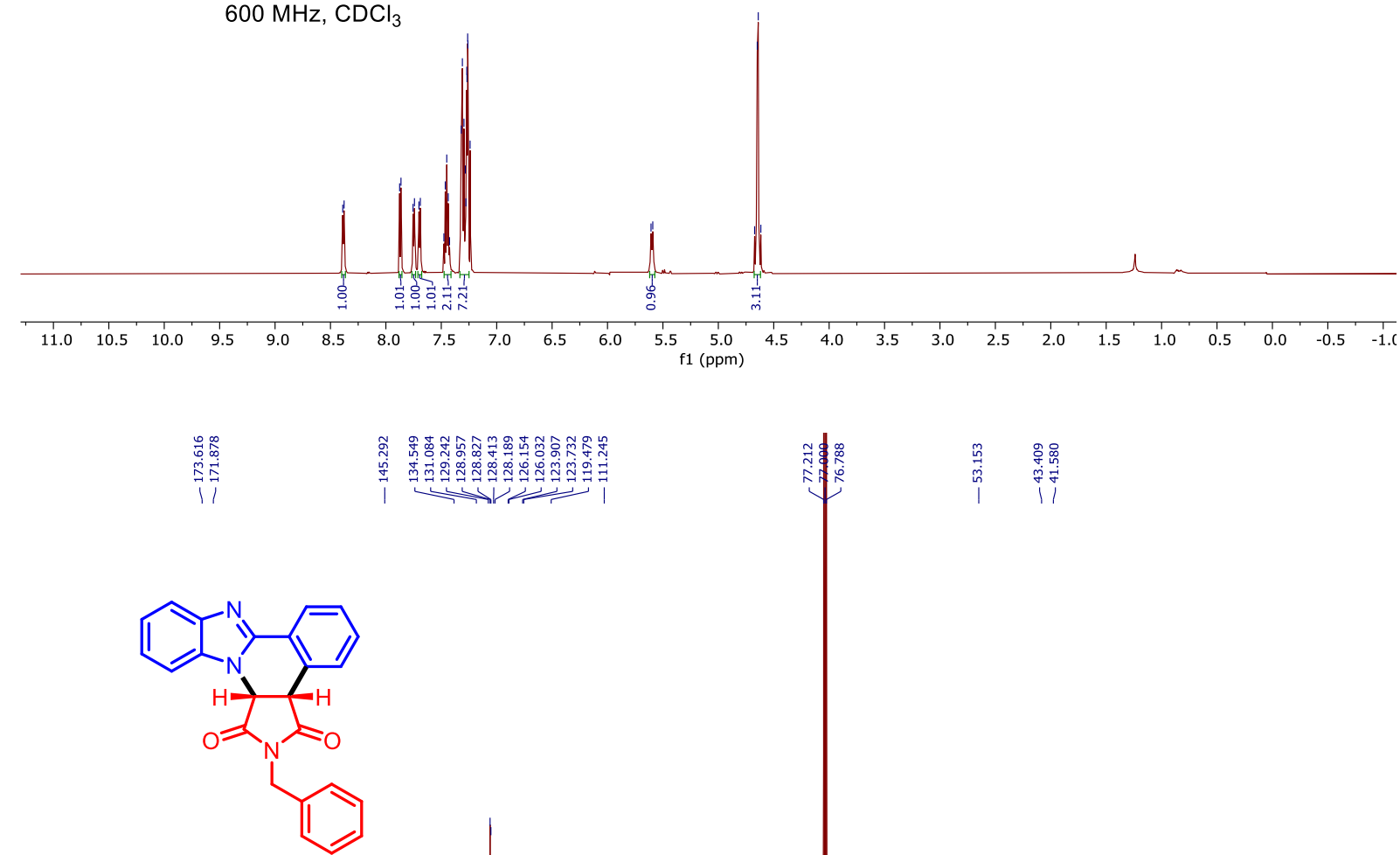

${ }^{13} \mathrm{C}$ NMR of $\mathbf{4 h}$ $150 \mathrm{MHz}, \mathrm{CDCl}_{3}$

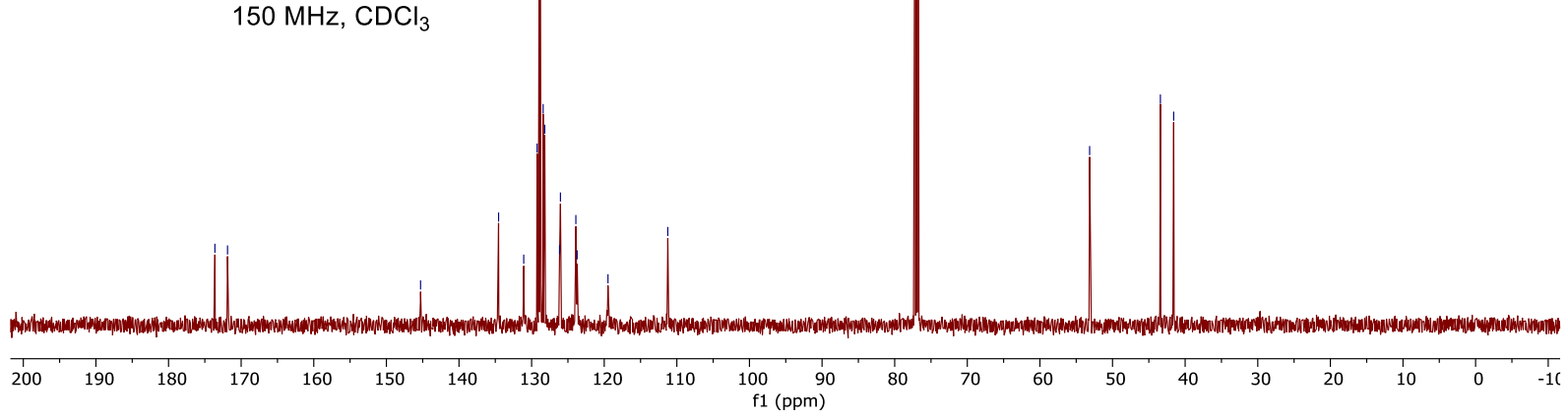




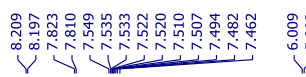

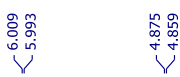

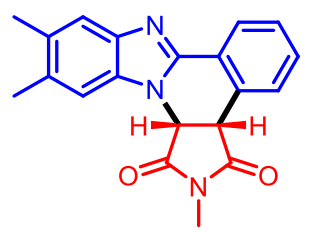

${ }^{1} \mathrm{H}$ NMR of $4 \mathbf{i}$

$600 \mathrm{MHz}, \mathrm{DMSO}-d_{6}$

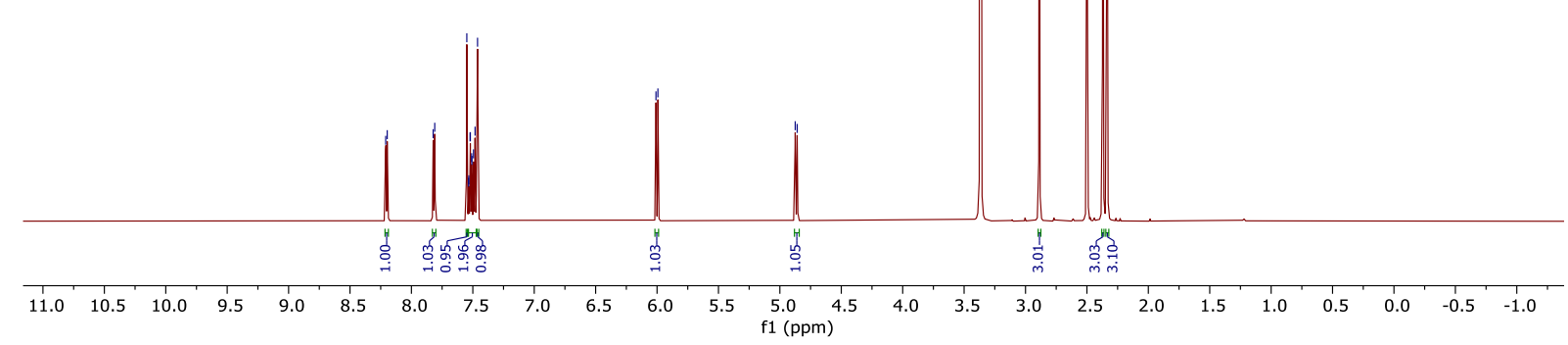

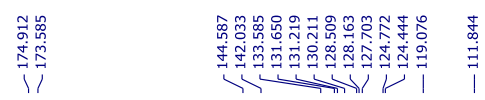

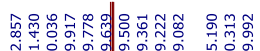

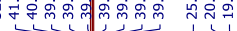

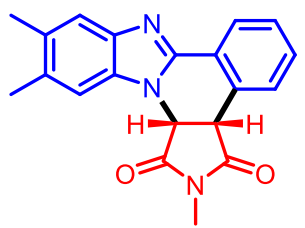

${ }^{13} \mathrm{C}$ NMR of $4 \mathbf{i}$

$150 \mathrm{MHz}$, DMSO- $d_{6}$

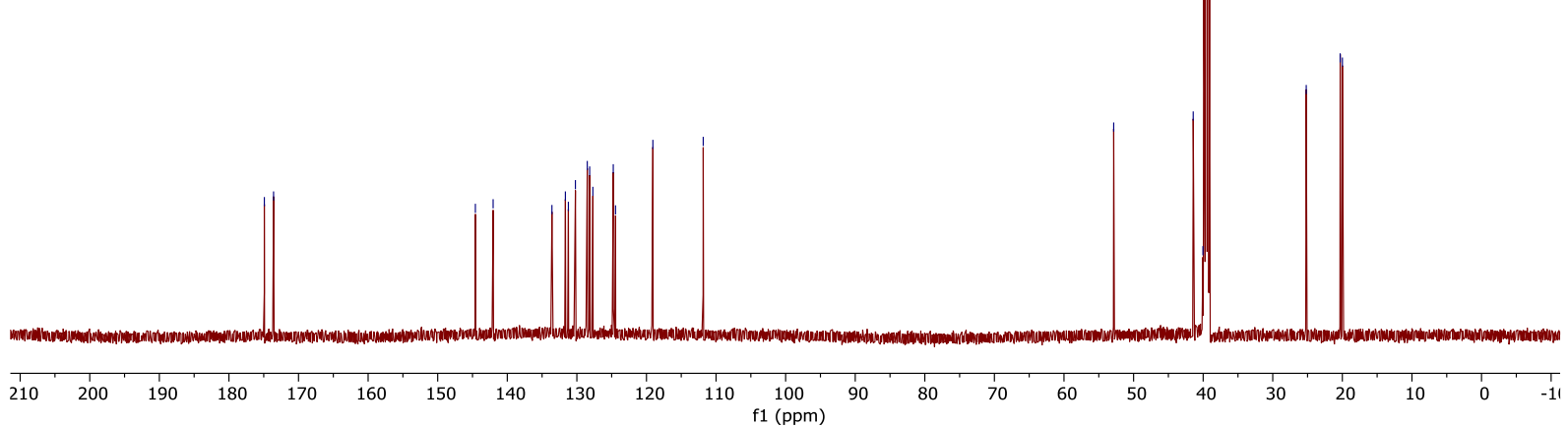



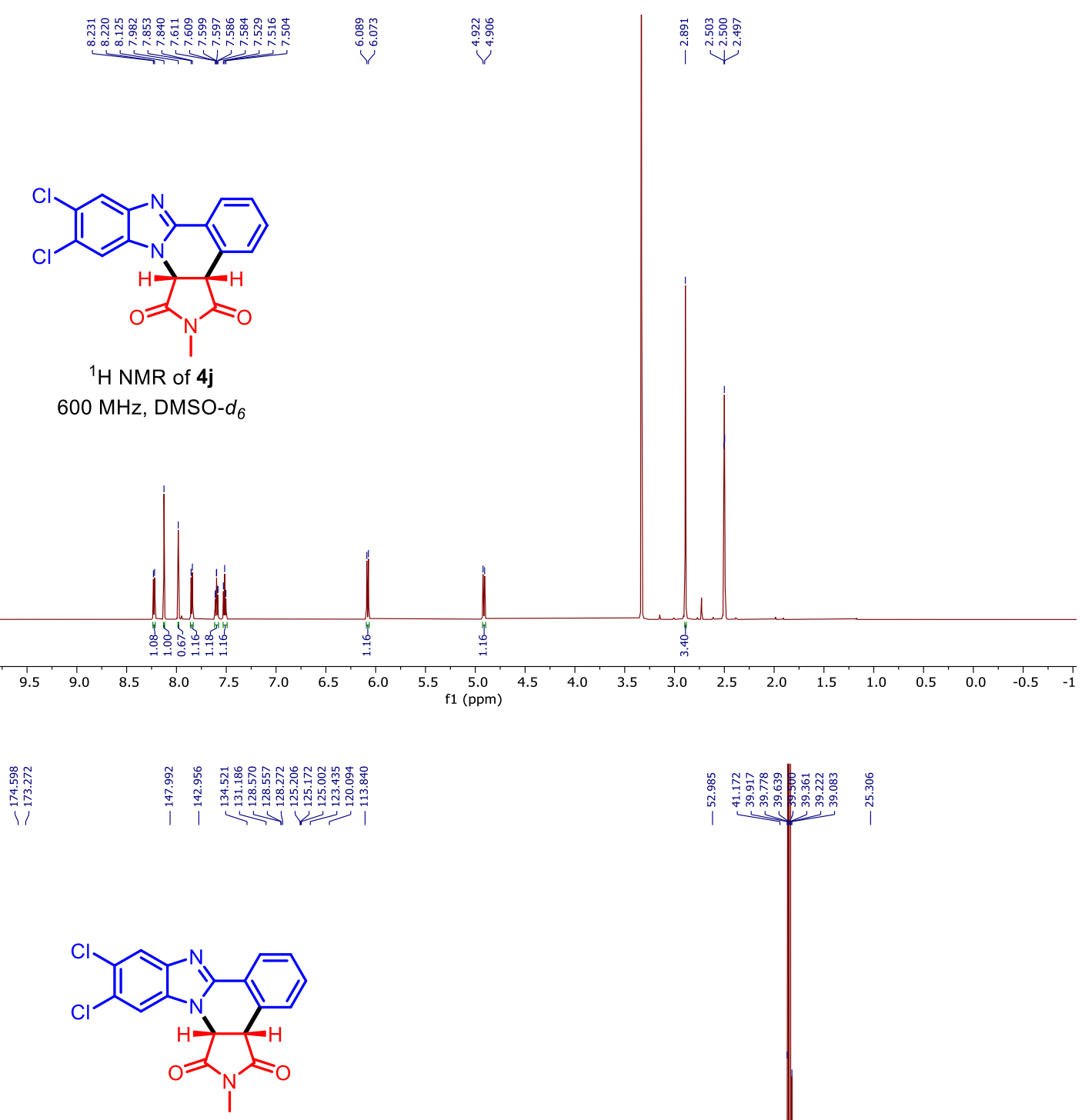

${ }^{13} \mathrm{C}$ NMR of $4 \mathbf{j}$

$150 \mathrm{MHz}, \mathrm{DMSO}-d_{6}$

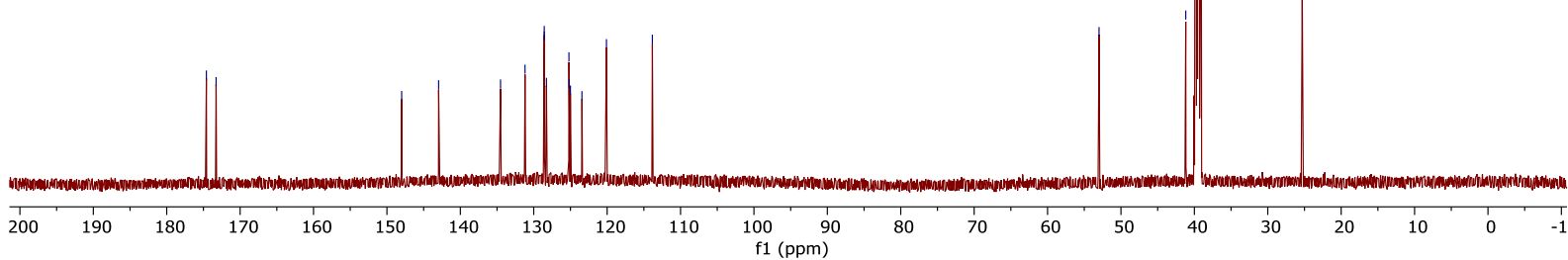



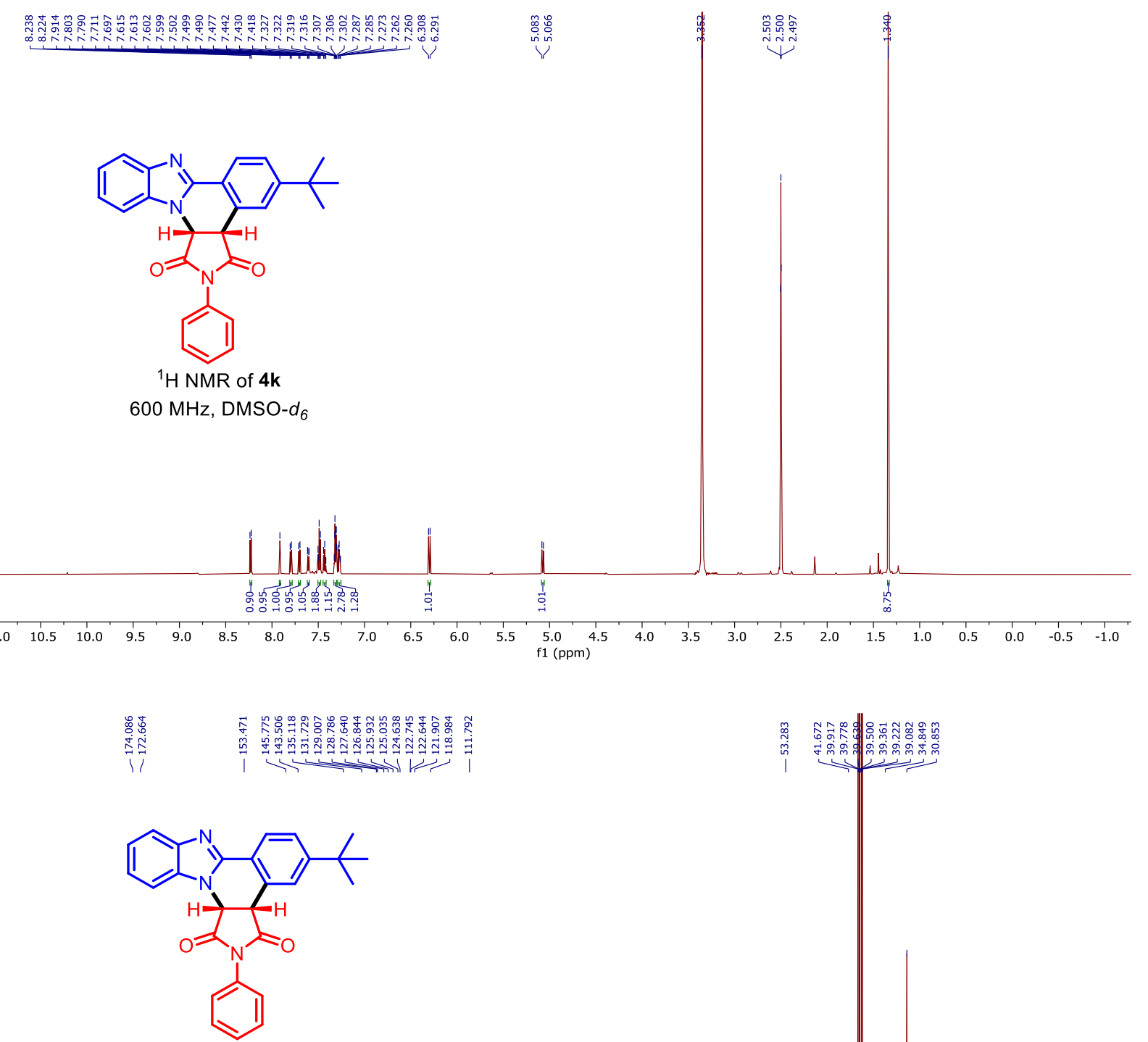

${ }^{13} \mathrm{C}$ NMR of $4 \mathbf{k}$

$150 \mathrm{MHz}, \mathrm{DMSO}-d_{6}$

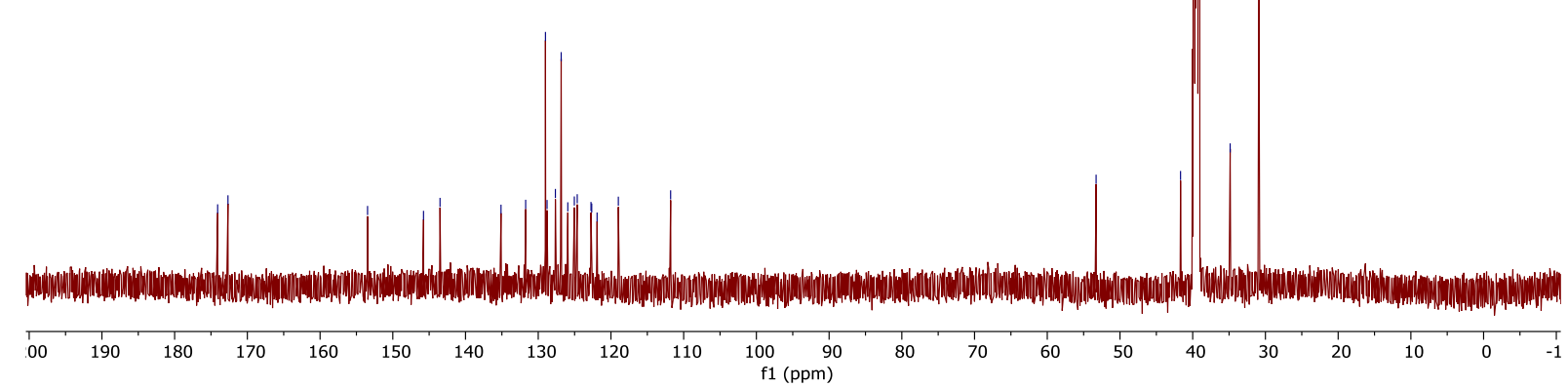




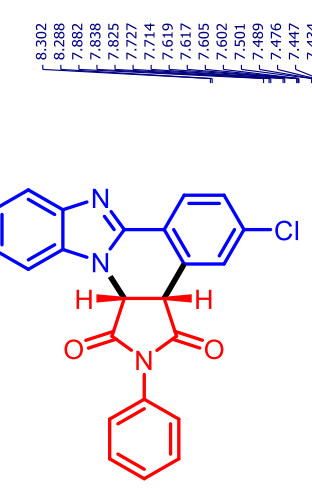

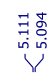

${ }^{1} \mathrm{H}$ NMR of 4

$600 \mathrm{MHz}, \mathrm{DMSO}-d_{6}$

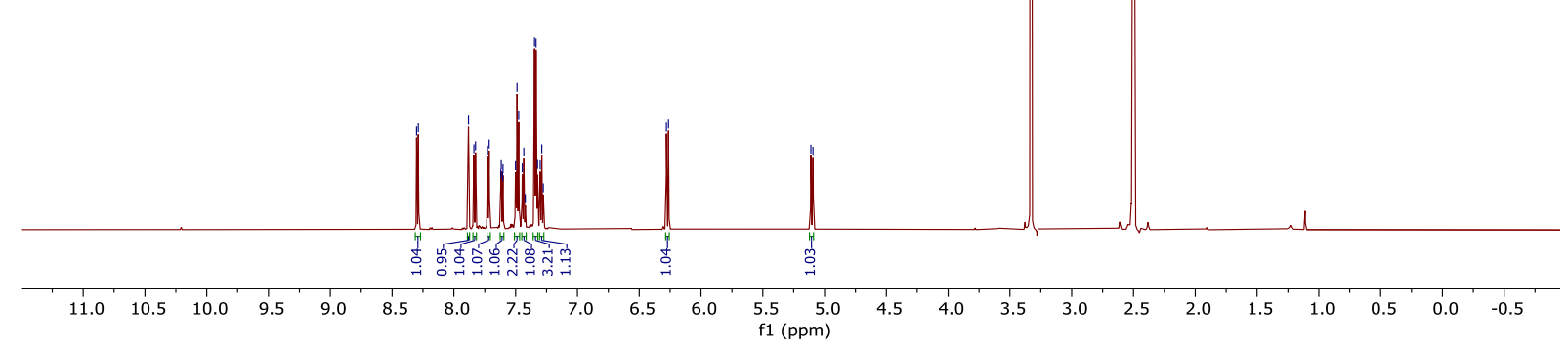

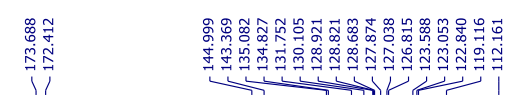

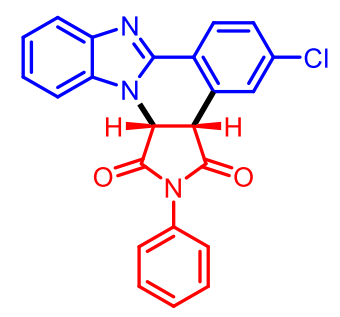

${ }^{13} \mathrm{C}$ NMR of $4 \mathrm{I}$

$150 \mathrm{MHz}$, DMSO- $d_{6}$

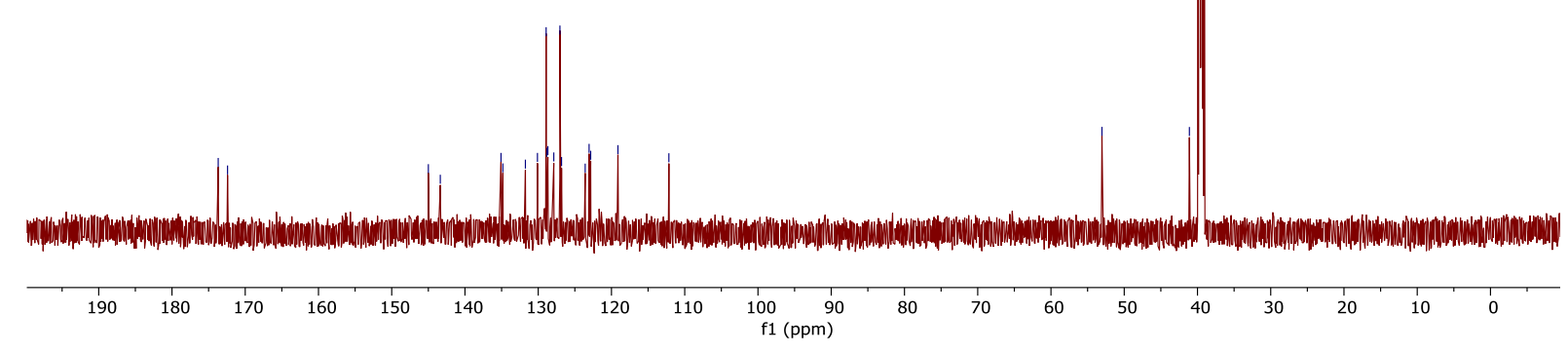



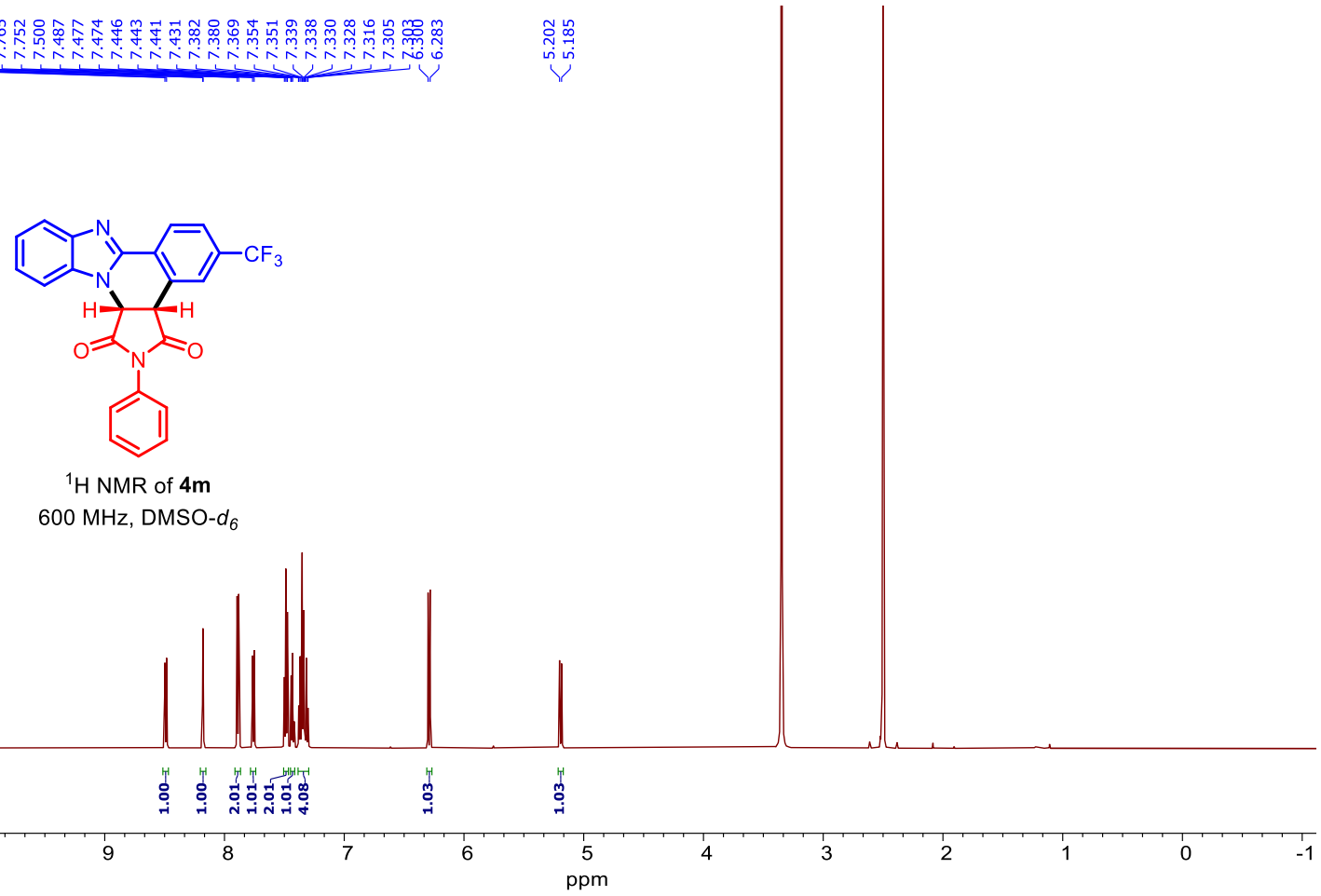

$600 \mathrm{MHz}$, DMSO- $d_{6}$
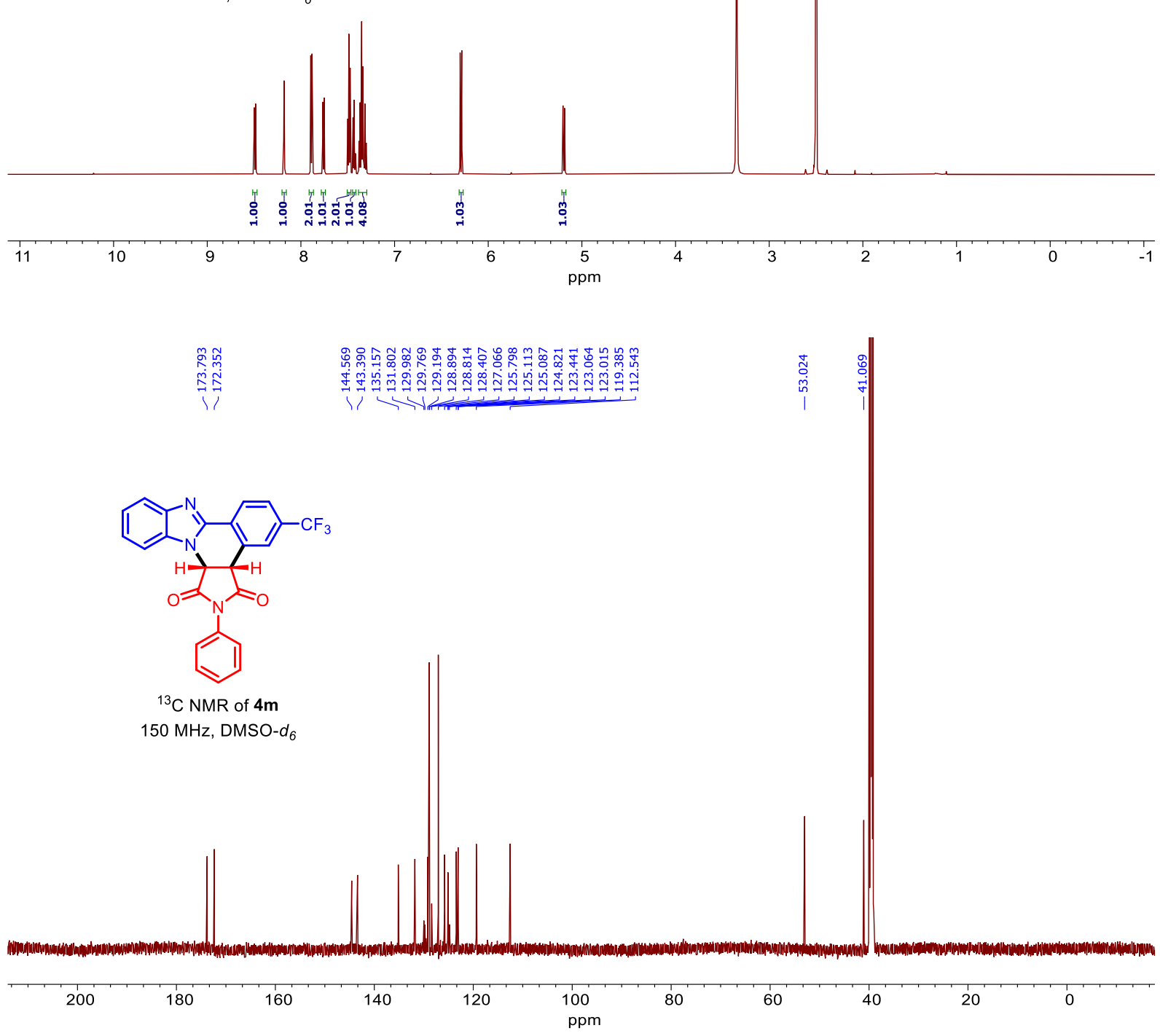


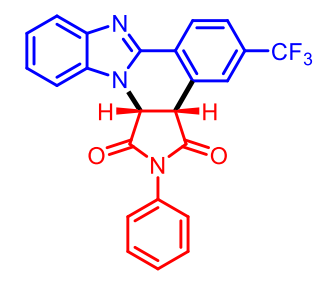

${ }^{19} \mathrm{~F}$ NMR of $4 \mathrm{~m}$ $565 \mathrm{MHz}$, DMSO- $d_{6}$

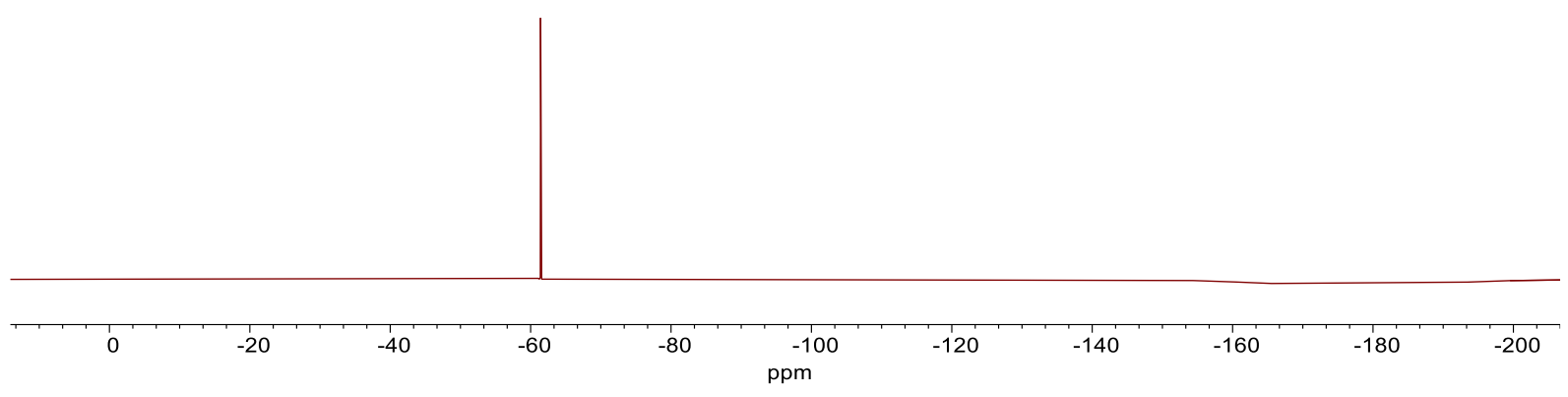




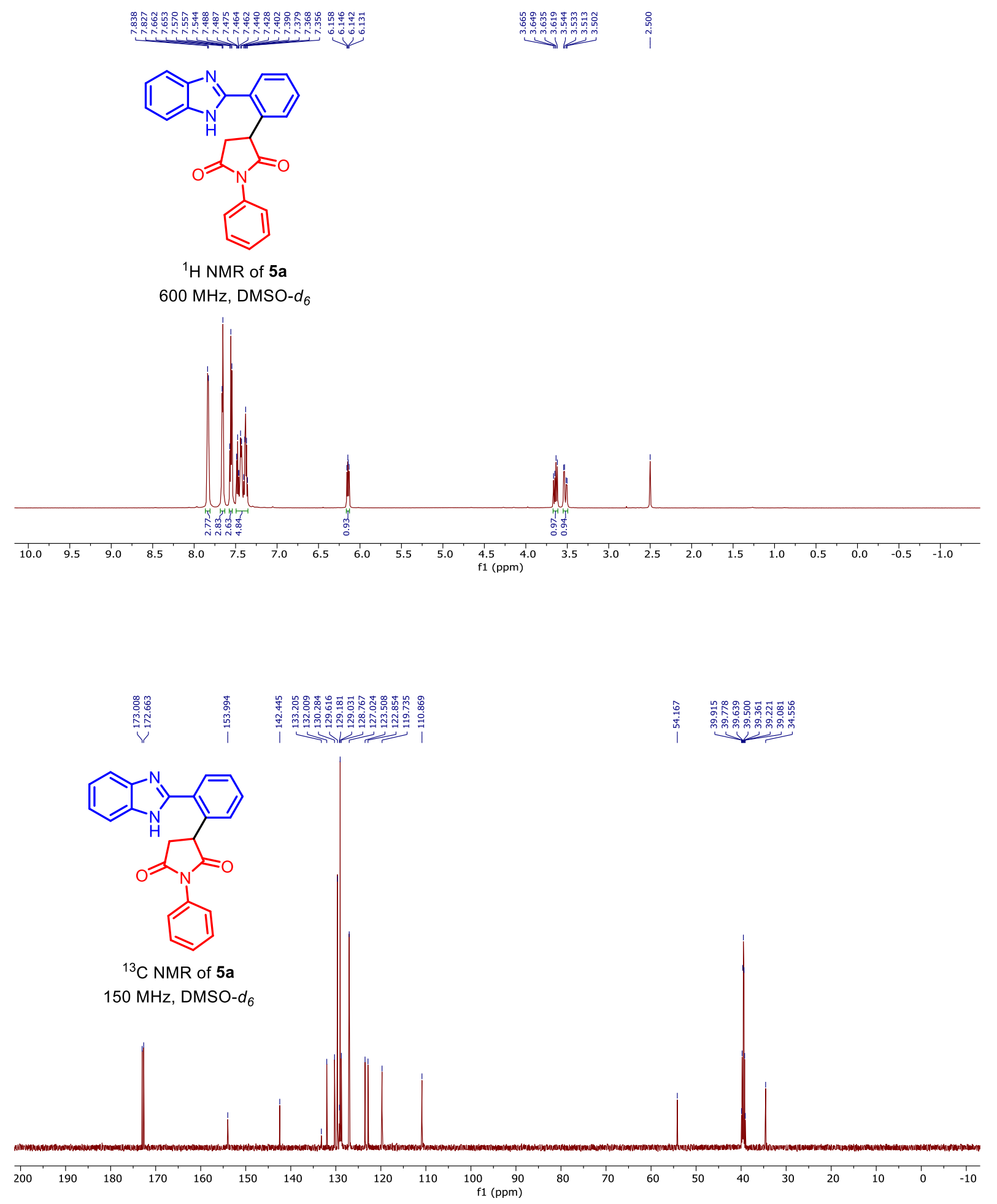




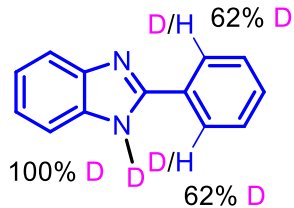

${ }^{1} \mathrm{H}$ NMR of 1a-D

$600 \mathrm{MHz}, \mathrm{DMSO}^{-d_{6}}$
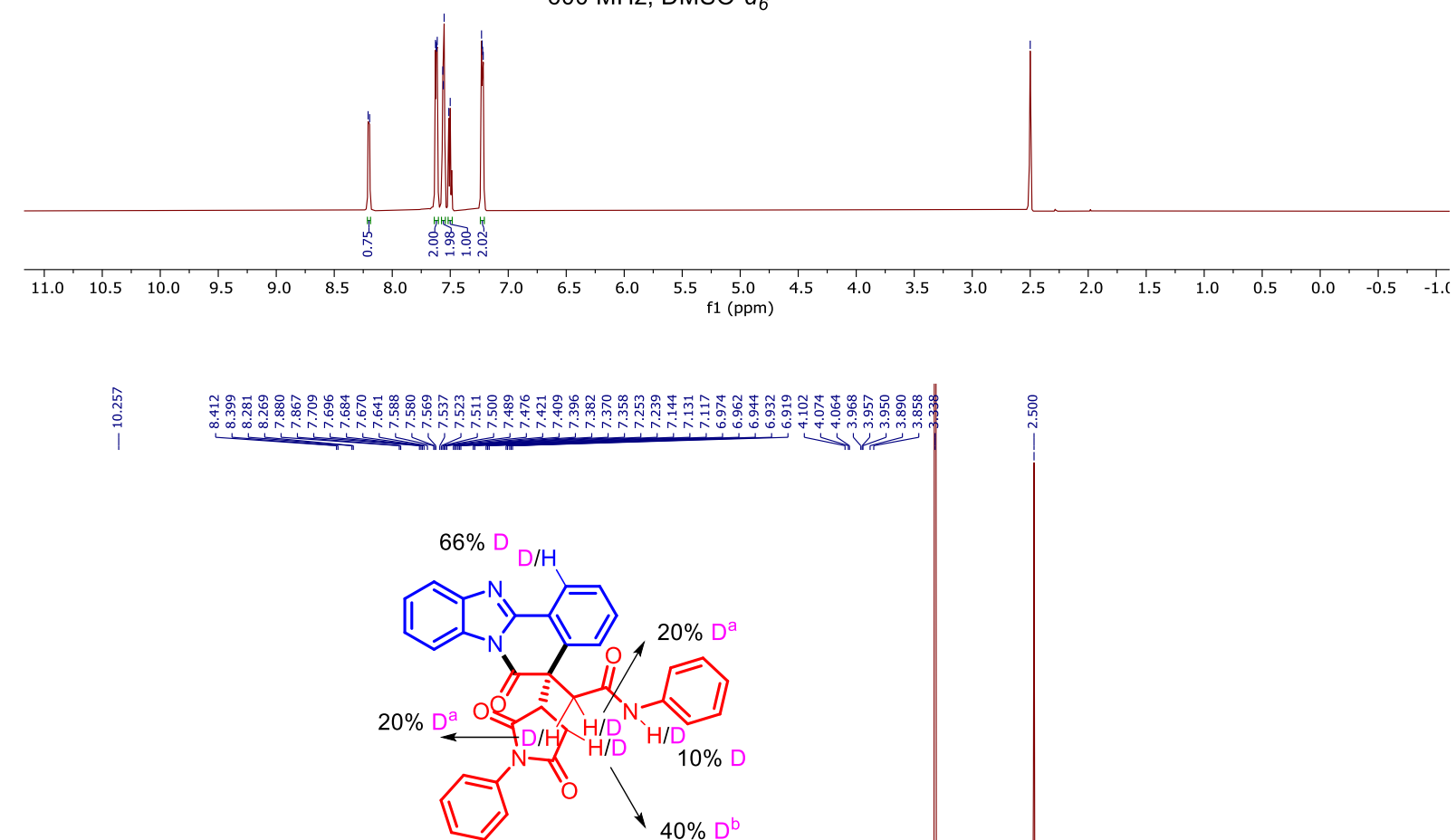

${ }^{1} \mathrm{H}$ NMR of 3a/3a-D

$600 \mathrm{MHz}$, DMSO- $d_{6}$

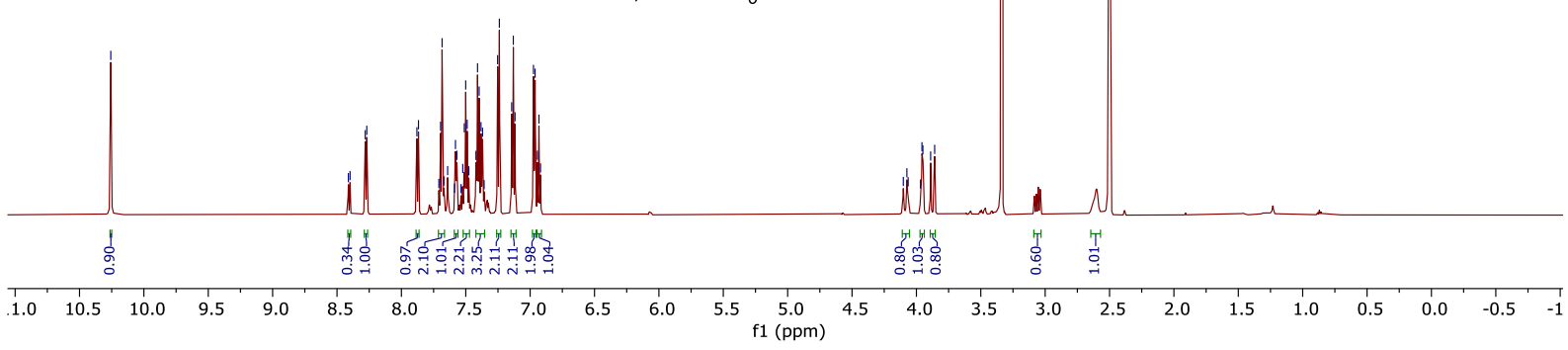




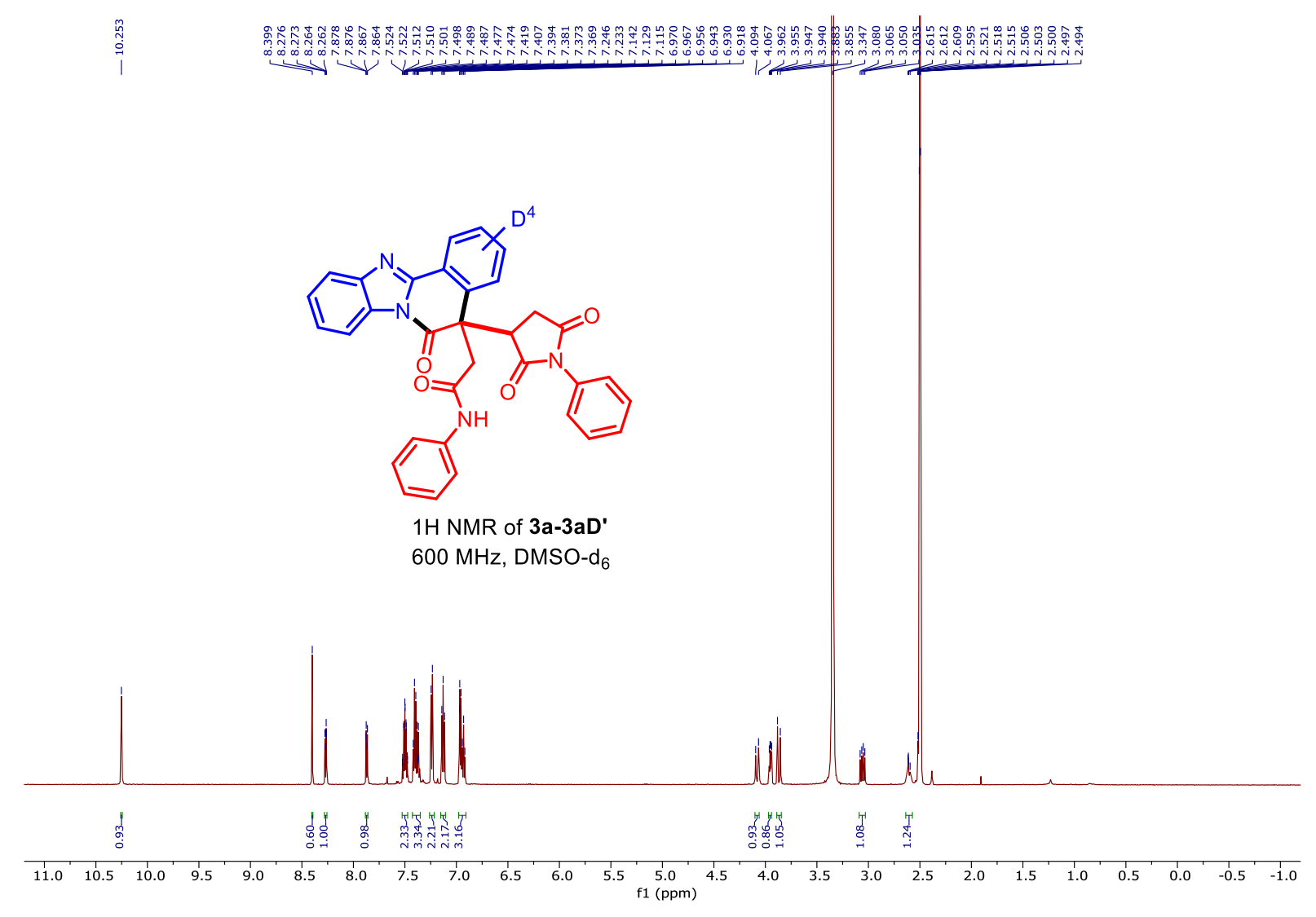




\section{Details of fluorophore analysis for heavy metal sensing}

\section{Procedure for optical properties with sensing measurements}

UV-Vis and fluorescence spectra were recorded using a UV 3220 spectrometer (Optizen) and spectrofluorometer (HITACHI) F-2700 equipped with a Xe arc lamp, respectively. A stock solution of $\mathbf{3 i}(\mathbf{L})(0.1 \mu \mathrm{M})$ was prepared in DMSO. Stock solutions of various cations $(20 \mu \mathrm{M}$; $\mathrm{Ag}^{+}, \mathrm{Al}^{3+}, \mathrm{Ba}^{2+}, \mathrm{Ca}^{2+}, \mathrm{Cd}^{2+}, \mathrm{Ce}^{3+}, \mathrm{Co}^{2+}, \mathrm{Cr}^{3+}, \mathrm{Cu}^{2+}, \mathrm{Fe}^{3+}, \mathrm{Hg}^{2+}, \mathrm{Ir}^{3+}, \mathrm{Mg}^{2+}, \mathrm{Mn}^{2+}, \mathrm{Na}^{+}, \mathrm{Ni}^{2+}$,

$\mathrm{Pb}^{2+}, \mathrm{Sn}^{2+}, \mathrm{Sr}^{2+}$, and $\mathrm{Zn}^{2+}$ ) were prepared in deionized water. Before the spectroscopic measurements, test solutions were prepared in $975 \mu \mathrm{L}$ of distilled water and $995 \mu \mathrm{L}$ of DMSO mixed with $5 \mu \mathrm{L}$ stock solution of $\mathbf{L}$ and $25 \mu \mathrm{L}$ of each metal ions stock into cuvettes with the final volume of $2.0 \mathrm{~mL}$ respectively. $\mathrm{Hg}^{2+}, \mathrm{Cu}^{2+}$, and $\mathrm{Fe}^{3+}$ metal ions were detected by adding a different aliquot of stock solution of $0-20 \mu \mathrm{M}$ with $\mathbf{L}$. All UV-Vis spectra were recorded at room temperature after the addition of samples for a few seconds. All the fluorescence measurements were carried out after 2 min of incubation at $328 \mathrm{~nm}\left(\lambda_{\text {exc }}\right)$ emission wavelength range from $300-600 \mathrm{~nm}$ with $5 \mathrm{~nm}$ of slit width, $250 \mathrm{~V}$ of photomultiplier tube voltage, and a scan speed of $240 \mathrm{~nm} / \mathrm{min}$.

\section{Fluorescent chemosensors for heavy metal sensing}

Heavy metal ions pollution has become a critical and urgent issue around the world due to the potential for harm to the human body even at very low concentrations. ${ }^{7}$ The development of chemosensors with highly selective and sensitive detection of the heavy metal ions considerable importance has raised concerns about potential negative effects on ecosystems in recent years. ${ }^{8}$ Among the heavy metal ions, mercury $\left(\mathrm{Hg}^{2+}\right)$ is known to be a highly toxic and widespread global pollutant, which is considered as one of the most poisonous and hazardous contaminants metal elements in the environment. ${ }^{9} \mathrm{Hg}^{2+}$ damages accumulation in the body can cause serious health problems, kidney failure, damaging central nervous, and leading to various cognitive and motion disorders. ${ }^{10}$ On the contrary, $\mathrm{Cu}^{2+}$ and $\mathrm{Fe}^{3+}$ ions are important environmental issues and critical trace elements in biological systems. ${ }^{11}$ However, high concentrations of $\mathrm{Cu}^{2+}$ and $\mathrm{Fe}^{3+}$ ions can be dangerous as highly reactive oxygen species $\left(\mathrm{Cu}^{2+}\right)$ and the direct oxidation of protein side chains $\left(\mathrm{Fe}^{3+}\right)$ and have been shown to nucleate protein aggregation linked to a variety of amyloid-based diseases, including Alzheimer's disease, Parkinson's disease, prion diseases, and dialysis-related amyloidosis. ${ }^{12}$ In contrast, since the 
diamagnetic property of $\mathrm{Hg}^{2+}$ and the paramagnetic property of $\mathrm{Cu}^{2+}$ and $\mathrm{Fe}^{3+}$ ions are dependent on fluorescence quenching mechanisms, ${ }^{13}$ it is still difficult to find a variety of metal ion sensors with high selectivity. In sensor design, achieving high selectivity for the analytes of interest in the presence of a complex background of potentially competing species is challenging work.

Chemosensors that respond to fluorescence properties to metal ions binding have a wide range of possible applications in biological, medical, and environmental research. The detailed experimental procedure and results and discussions for the fluorophore with sensing analysis are described. Briefly, the optical properties such as absorption and emission spectra of the synthesized compounds $\mathbf{3 b}-\mathbf{3 t}$ and $\mathbf{4 a - 4 k}$ was carried out in mixed aqueous solution DMSO/ $\mathrm{H}_{2} \mathrm{O}(5 / 5, \mathrm{v} / \mathrm{v})$ (Figs. S2, S3). Among the tested compounds, 3i (L) is a highly fluorescent active, which can bind with metal ions due to the presence of different active sites, such as carbonyl $(\mathrm{C}=\mathrm{O})$, amine $(-\mathrm{N}-)$ groups. Correspondingly, the absorption and fluorescence response of the $\mathbf{L}$ toward 20 different metal ions in mixed aqueous solutions, a shifted excitation and emission maxima with quenching in fluorescence intensity of $\mathbf{L}$ was observed by the addition of $\mathrm{Hg}^{2+}$ (quenching at $355 \mathrm{~nm}$ ), $\mathrm{Cu}^{2+}$ (quenching with red-shifted from $355 \mathrm{~nm}$ to $360 \mathrm{~nm}$ ), and $\mathrm{Fe}^{3+}$ (quenching with red-shifted from $355 \mathrm{~nm}$ to $371 \mathrm{~nm}$ ) ions, when excited at $328 \mathrm{~nm}$ (Fig. S4a,b). It was found that in response to the highly selective "turn-off" complex formation of $\mathbf{L}-\mathrm{Hg}^{2+}, \mathbf{L}-\mathrm{Cu}^{2+}$, and $\mathbf{L}-\mathrm{Fe}^{3+}$, respectively. ${ }^{14}$ In comparison, no effect on the absorption and fluorescence of $\mathbf{L}$ was observed with the addition of several metal ions (Fig. $\mathrm{S} 4 \mathrm{a}, \mathrm{b})$. We decided that the sensor will most likely chelate $\mathrm{Hg}^{2+}, \mathrm{Cu}^{2+}$, and $\mathrm{Fe}^{3+}$ metal ions through the $\mathrm{O}$ and $\mathrm{N}$ atoms, which are close to those of previously reported sensors. ${ }^{15}$ Subsequently, a fluorescence titration experiment was carried out to further sensitivity of $\mathbf{3 i}$ (L) toward $\mathrm{Hg}^{2+}, \mathrm{Cu}^{2+}$, and $\mathrm{Fe}^{3+}$ ions by the various concentrations of 0-20 $\mu \mathrm{M}$ (Fig. S4a-c). It has been found that the "turn-off" quenching fluorescence intensity of $\mathbf{L}$ linearly with the incremental addition of the $\mathrm{Hg}^{2+}, \mathrm{Cu}^{2+}$, and $\mathrm{Fe}^{3+}$ metal ions, which indicates that the $\mathbf{L}$ is strongly binding to the metal ions. A plot of the linear Stern-Volmer equation, ${ }^{16}$ the measured fluorescence intensity $\left[\mathrm{F}_{0} / \mathrm{F}\right]$ varied as a function of $\mathrm{Hg}^{2+}, \mathrm{Cu}^{2+}$, and $\mathrm{Fe}^{3+}$ concentration that showed good linearity with correlation coefficients $\left(\mathrm{R}^{2}=0.9944,0.9909\right.$, and 0.8979, respectively) (inset Fig. S5a-c). Thus, following the 1:1 and 2:1 stoichiometry, the possible binding mode between $\mathbf{L}$ toward $\mathrm{Hg}^{2+}, \mathrm{Cu}^{2+}$, and $\mathrm{Fe}^{3+}\left(\mathbf{L}-\mathrm{Hg}^{2+}, \mathbf{L}-\mathrm{Cu}^{2+}\right.$, and $\left.\mathbf{2} \mathbf{L}-\mathrm{Fe}^{3+}\right)$ were proposed interaction mechanisms (See Scheme S2). The limit of detection was calculated based 
on the equation $(\mathrm{LOD}=\mathrm{K} \times \mathrm{SD} / \mathrm{S}$ ) as $85.9 \mathrm{nM}, 84.8 \mathrm{nM}$, and $60.0 \mathrm{nM}$ with a linear range of 0-20 $\mu \mathrm{M}$ of metal complexes $\mathbf{L}-\mathrm{Hg}^{2+}, \mathbf{L}-\mathrm{Cu}^{2+}$, and $\mathbf{2} \mathbf{L}-\mathrm{Fe}^{3+}$, respectively (Fig. S6a-c).
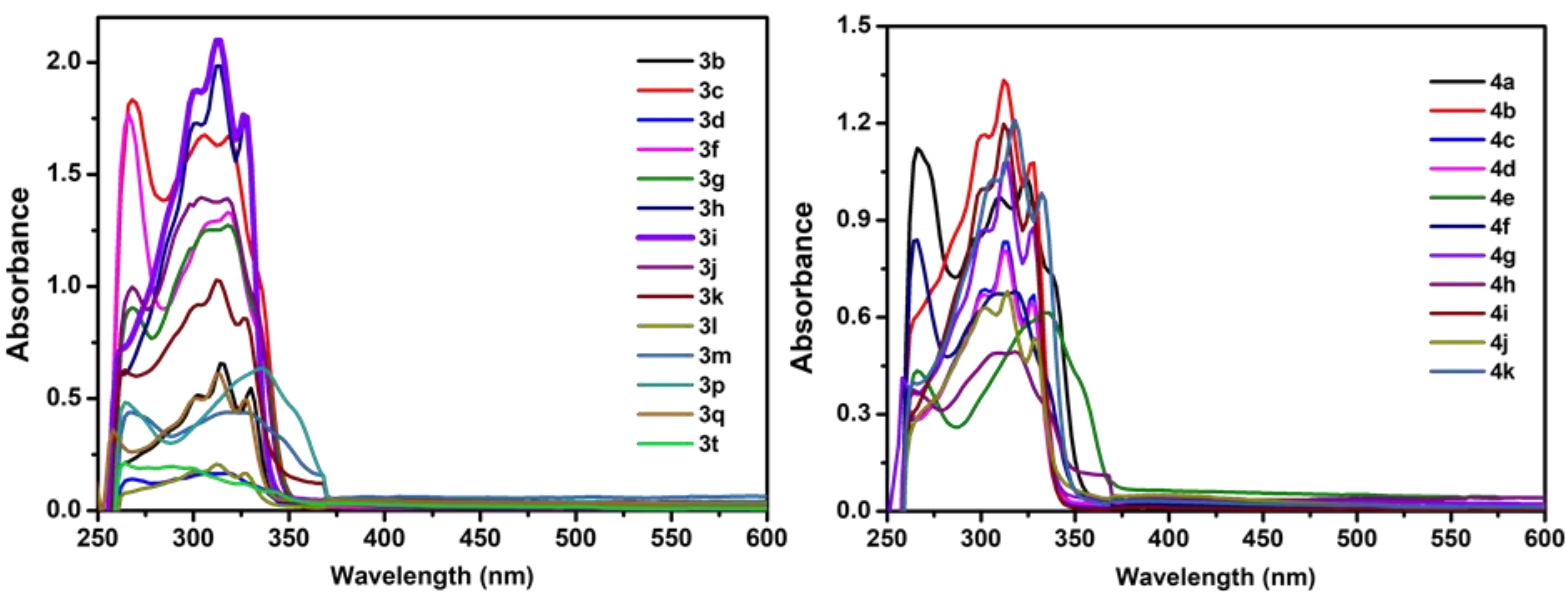

Fig. S2. UV-Visible spectral properties of $\mathbf{3 b - 3 t}$ and $\mathbf{4 a - 4 k}$.
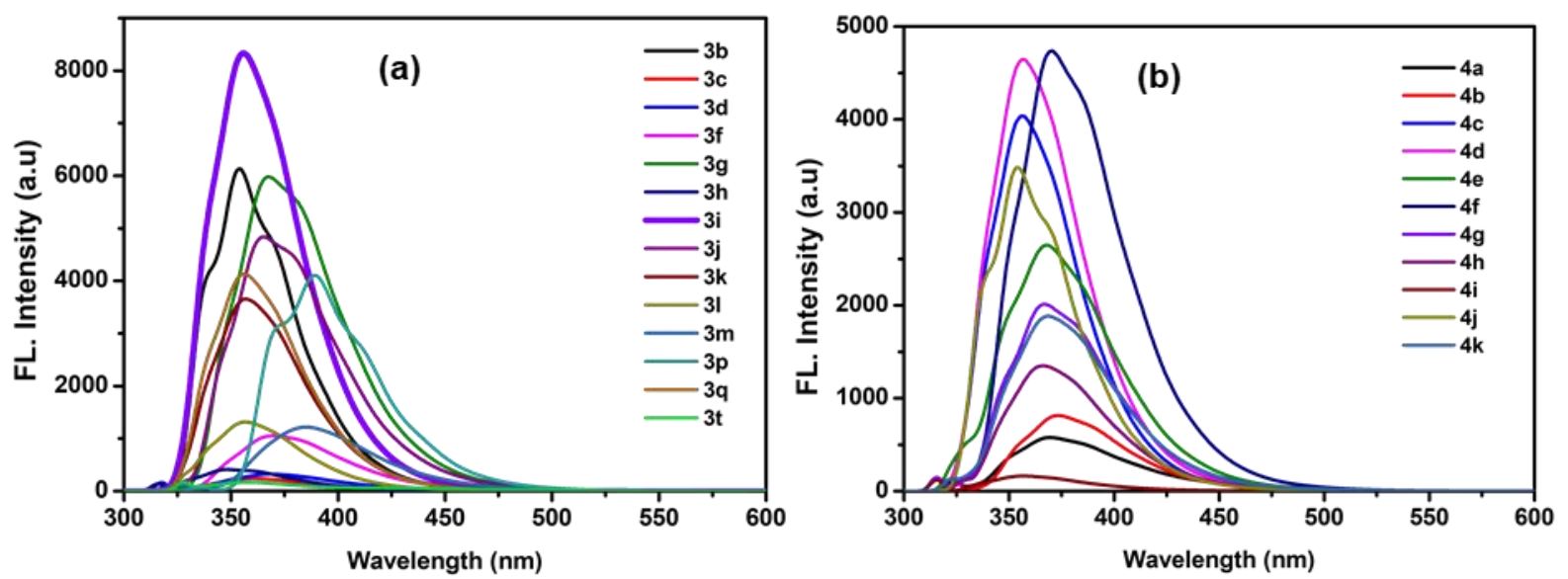

Fig. S3. Fluorescence spectral properties of $3 \mathbf{b}-\mathbf{3 t}$ and $\mathbf{4 a - 4 k}$. 

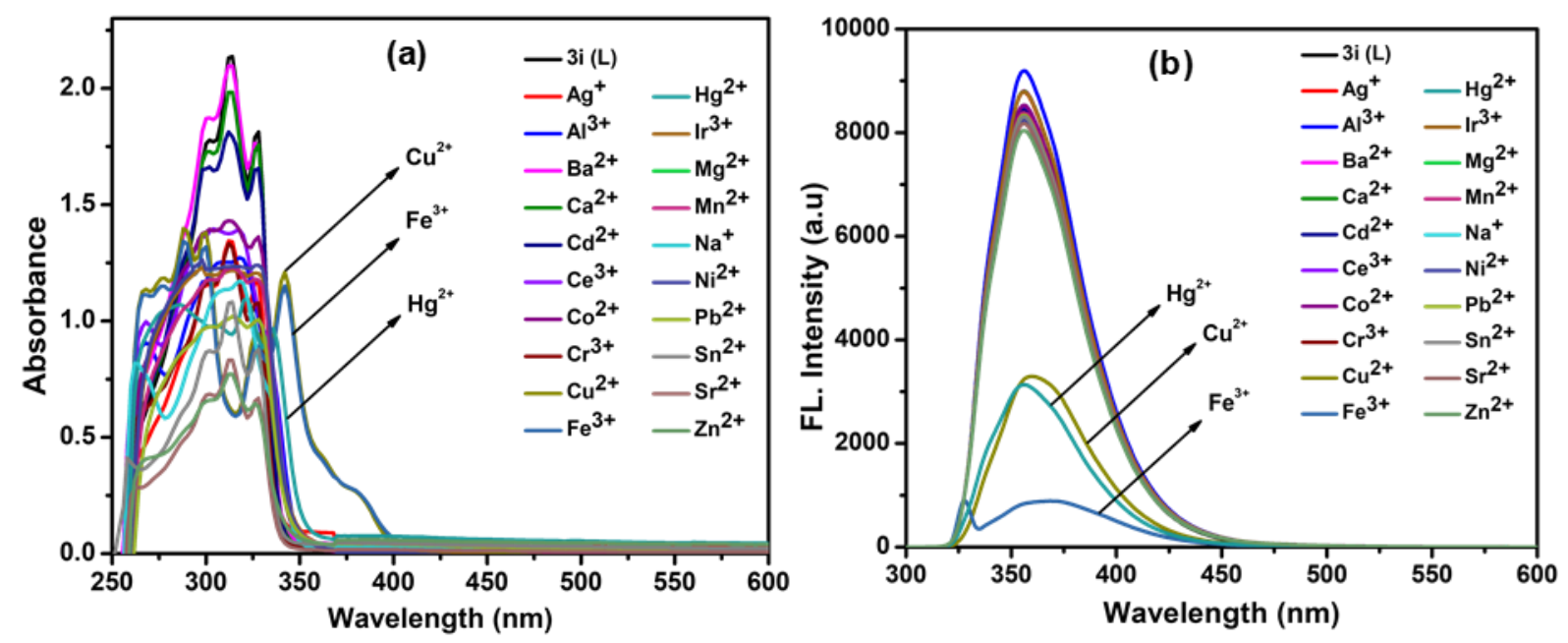

Fig. S4. (a) UV-Visible and (b) Fluorescence spectra of $\mathbf{L}(0.1 \mu \mathrm{M})$ with the addition of various metal ions $(20 \mu \mathrm{M})$ 

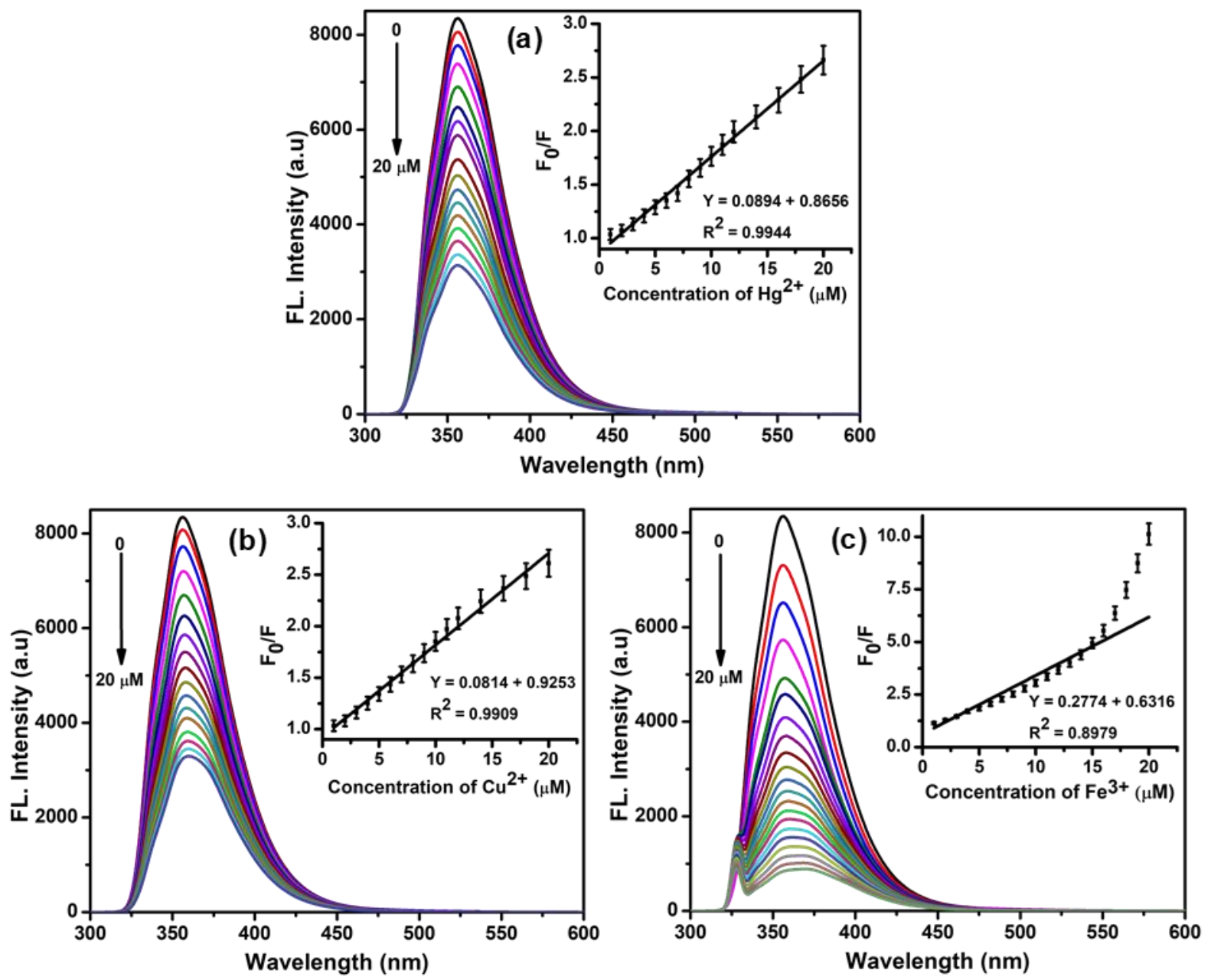

Fig. S5. Fluorescence spectra of $\mathbf{L}(0.1 \mu \mathrm{M})$ following the addition of $0-20 \mu \mathrm{M}$ of (a) $\mathrm{Hg}^{2+}$, (b) $\mathrm{Cu}^{2+}$, and (c) $\mathrm{Fe}^{3+}$ metal ions. All the spectra used in the solvent mixture of $\mathrm{DMSO} / \mathrm{H}_{2} \mathrm{O}(5 / 5$, $\mathrm{v} / \mathrm{v}$ ) at room temperature. Excitation wavelength: $328 \mathrm{~nm}$. The inset figure in linear SternVolmer plot of $\mathrm{F}_{0} / \mathrm{F}$ versus (a) $\mathrm{Hg}^{2+}$, (b) $\mathrm{Cu}^{2+}$, and (c) $\mathrm{Fe}^{3+}$ metal ions concentration. 

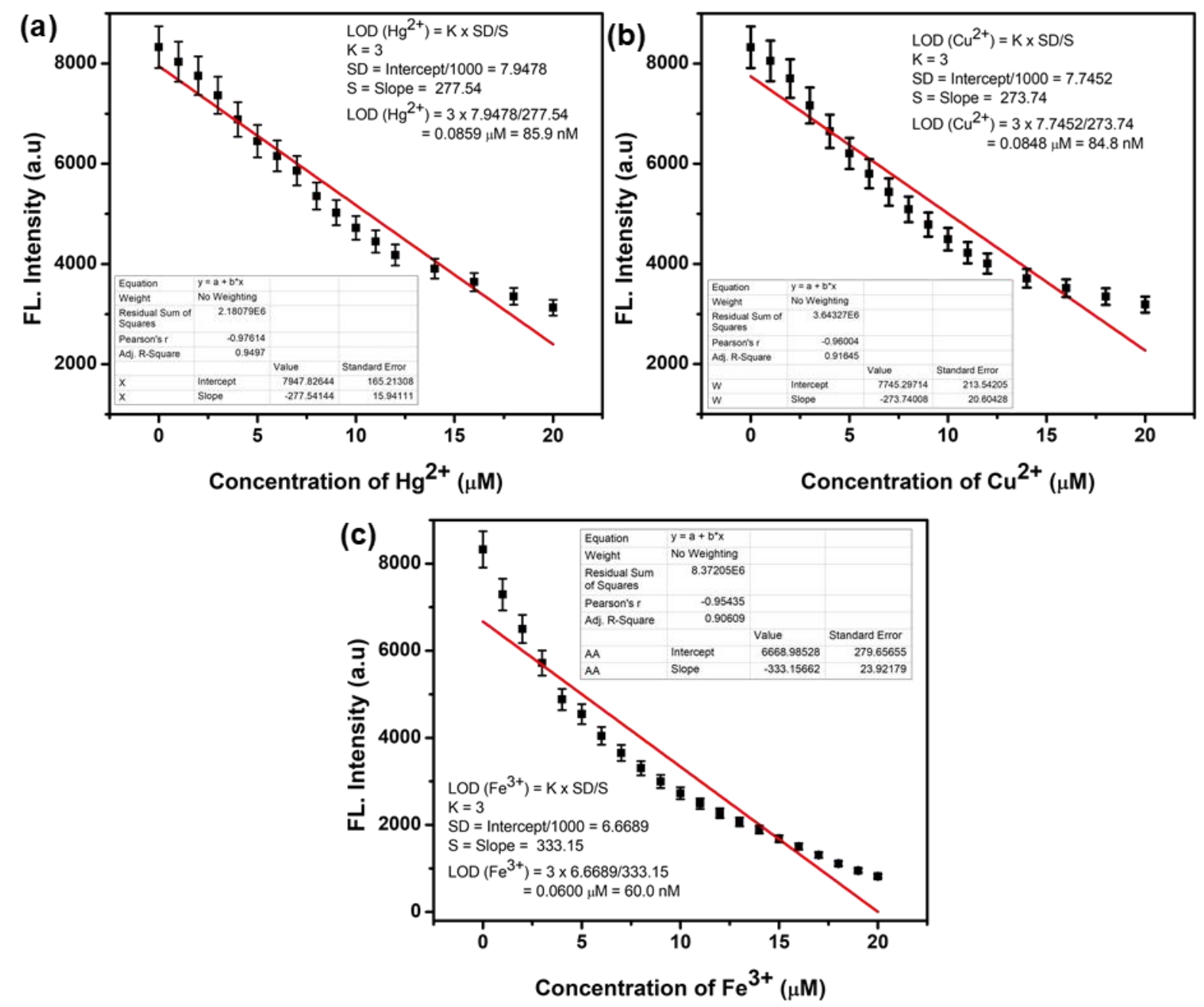

Fig. S6. Calibration curve of $\mathbf{L}(0.1 \mu \mathrm{M})$ in the presence of (a) $\mathrm{Hg}^{2+}$, (b) $\mathrm{Cu}^{2+}$, and (c) $\mathrm{Fe}^{3+}$ metal ions using the monitored emission wavelength at $355 \mathrm{~nm}$ in all these systems. The limit of detection (LOD) was determined from the following equation: $\mathrm{LOD}=\mathrm{K} \times \mathrm{SD} / \mathrm{S}$, where $\mathrm{K}$ $=3 ; \mathrm{SD}$ is the standard deviation of the blank solution; $\mathrm{S}$ is the slope of the calibration curve. 


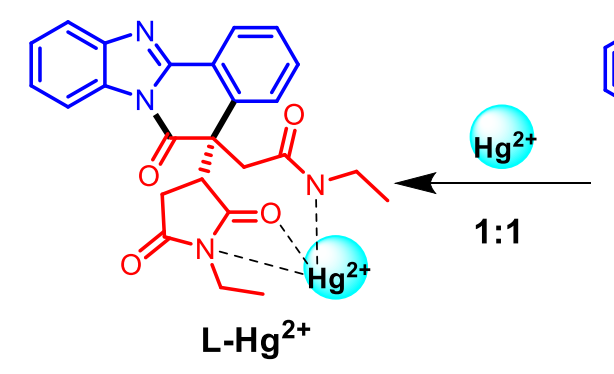

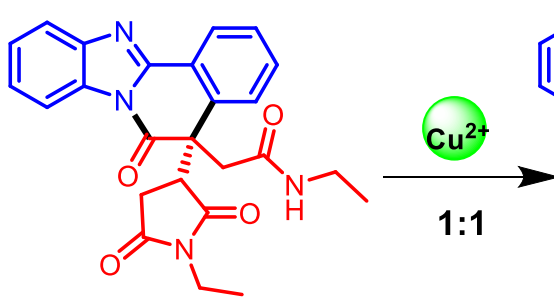

$\mathbf{L}$

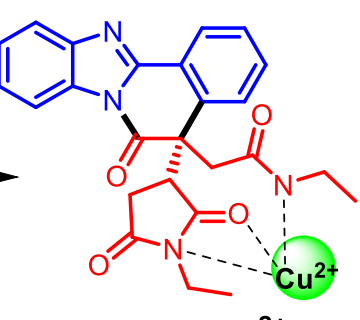

$\mathrm{L}-\mathrm{Cu}^{2+}$

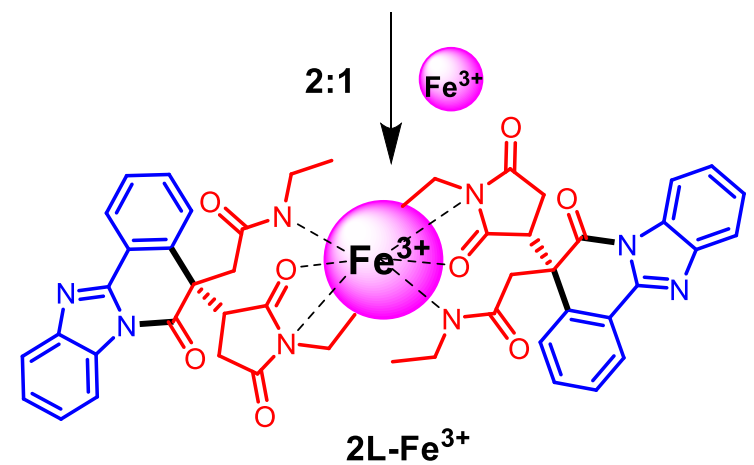

Scheme S2. The Proposed Complexation Mechanism of Fluorescent Chemosensor $\mathrm{L}$ with $\mathrm{Hg}^{2+}, \mathrm{Cu}^{2+}$, and $\mathrm{Fe}^{3+}$ Metal Ions.

\section{References:}

1. Hikawa, H.; Ichinose, R.; Kikkawa, S.; Azumaya, I. Palladium-Catalyzed Dehydrogenation of Benzyl Alcohols for Construction of 2-Arylbenzimidazoles "On Water". Asian J. Org. Chem. 2018, 7, 416.

2. Gan, H. Fe/S-Catalyzed Redox Condensation of $o$-Nitrophenols/Thiophenols/Anilines with Dibenzyl Disulfide: Access to 2-Arylbenzazoles. ChemistrySelect 2020, 5, 717.

3. Zuo, M.; Guo, W.; Pang, Y.; Guo, R.; Hou, C.; Sun, S.; Wu, H.; Sun, Z.; Chu, W. Direct Synthesis of 2-Substituted Benzimidazoles via Dehydrogenative Coupling of Aromatic-Diamine and Primary Alcohol Catalyzed by a Co Complex. New J. Chem. 2020, 44, 14490.

4. Saha, M.; Mukherjee, P.; Das, A. R. Practical Application of $\mathrm{PhI}(\mathrm{OAc})_{2} / \mathrm{I}_{2}$ Combination to Synthesize Benzimidazoles from 2-Aminobenzylamine through Ring Distortion Strategy. Tetrahedron Lett. 2017, 58, 1046.

5. Wang, Y.; Sarris, K., Sauer, D. R.; Djuric, S. W. A Simple and Efficient One Step Synthesis of Benzoxazoles and Benzimidazoles from Carboxylic Acids. Tetrahedron Lett. 2006, 47, 4823. 
6. Kalgutkar, A. S.; Crews, B. C.; Marnett, L. J. Design, Synthesis, and Biochemical Evaluation of $\mathrm{N}$-Substituted Maleimides as Inhibitors of Prostaglandin Endoperoxide Synthases. J. Med. Chem. 1996, 39, 1692.

7. Briffa, J.; Sinagra, E.; Blundell, R. Heavy Metal Pollution in the Environment and Their Toxicological Effects on Humans. Heliyon 2020, 6, e04691.

8. (a) Mon, M.; Lloret, F.; Ferrando-Soria, J.; Martí-Gastaldo, C.; Armentano, D.; Pardo, E., Selective and Efficient Removal of Mercury from Aqueous Media with the Highly Flexible Arms of a BioMOF. Angew. Chem. Int. Ed. 2016, 128, 11333. (b) Liu, D.; Deng, S.; Du, R.; Tao, L.; Sun, J.; Yu, G. Efficient and Selective Removal of Copper from Aqueous Solution by Nanosized Hydrated Zirconium Oxides Loaded in Vermiculite. J. Environ. Chem. Eng. 2020, 104315. (c) Lv, R.; Hu, Y.; Jia, Z.; Li, R.; Zhang, X.; Liu, J.; Fan, C.; Feng, J.; Zhang, L.; Wang, Z.; Removal of $\mathrm{Fe}^{3+}$ and $\mathrm{Al}^{3+}$ Ions from Phosphoric Acid-Nitric Acid Solutions with Chelating Resins. Hydrometallurgy 2019, 188, 194.

9. Tchounwou, P. B.; Ayensu, W. K.; Ninashvili, N.; Sutton, D. Environmental Exposure to Mercury and its Toxicopathologic Implications for Public Health. Environ. Toxicol. 2003, 18, 149.

10. (a) Culzoni, M. J.; De La Peña, A. M.; Machuca, A.; Goicoechea, H. C.; Babiano, R.; Rhodamine and BODIPY Chemodosimeters and Chemosensors for the Detection of $\mathrm{Hg}$ ${ }^{2+}$, Based on Fluorescence Enhancement Effects. Analytical Methods 2013, 5, 30-49. (b) Saha, S.; Mahato, P.; Suresh, E.; Chakrabarty, A.; Baidya, M.; Ghosh, S. K.; Das, A. Recognition of $\mathrm{Hg}^{2+}$ and $\mathrm{Cr}^{3+}$ in Physiological Conditions by a Rhodamine Derivative and its Application as a Reagent for Cell-Imaging Studies. Inorganic chemistry 2012, 5, 336.

11. Zheng, X.; Cheng, W.; Ji, C.; Zhang, J.; Yin, M. Detection of Metal Ions in Biological Systems: A Review. Crit. Rev. Anal. Chem. 2020, 39, 231.

12. (a) Taylor, A. B.; Stoj, C. S.; Ziegler, L.; Kosman, D. J.; Hart, P. J. The Copper-Iron Connection in Biology: Structure of the Metallo-Oxidase Fet3p. PNAS, 2005, 102, 15459. (b) Liu, T.; Ramesh, A.; Ma, Z.; Ward, S. K.; Zhang, L.; George, G. N.; Talaat, A. M.; Sacchettini, J. C.; Giedroc, D. P. CsoR is a Novel Mycobacterium Tuberculosis Copper-Sensing Transcriptional Regulator. Nat. Chem. Biol. 2007, 3, 60.

13. (a) Choi, Y. W.; Lee, J. J.; You, G. R.; Kim, C. Fluorescence ‘on-off-on' Chemosensor for the Sequential Recognition of $\mathrm{Hg}^{2+}$ and Cysteine in Water. RSC Adv. 2015, 5, 
38308. (b) Tamil Selvan, G.; Varadaraju, C.; Tamil Selvan, R.; Enoch, I. V.; Mosae Selvakumar, P.; On/off Fluorescent Chemosensor for Selective Detection of Divalent Iron and Copper Ions: Molecular Logic Operation and Protein Binding. ACS omega 2018, 3, 7985. (c) He, W.; Liu, Z. A Fluorescent Sensor for $\mathrm{Cu}^{2+}$ and $\mathrm{Fe}^{3+}$ Based on Multiple Mechanisms. RSC Adv. 2016, 6, 59073.

14. (a) Choi, Y. W.; Lee, J. J.; You, G. R.; Kim, C. Fluorescence 'on-off-on' Chemosensor for the Sequential Recognition of $\mathrm{Hg}^{2+}$ and Cysteine in Water. RSC Adv. 2015, 5, 38308. (b) He, W.; Liu, Z. A Fluorescent Sensor for $\mathrm{Cu}^{2+}$ and $\mathrm{Fe}^{3+}$ Based on Multiple Mechanisms. RSC Adv. 2016, 6, 59073.

15. (a) Yang, Y.; Gao, C.; Li, B.; Xu, L.; Duan, L. A Rhodamine-Based Colorimetric and Reversible Fluorescent Chemosensor for Selectively Detection of $\mathrm{Cu}^{2+}$ and $\mathrm{Hg}^{2+}$ Ions. Sens. Actuators B Chem. 2014, 199, 121. (b) Yin, P.; Niu, Q.; Liu, J.; Wei, T.; Hu, T.; Li, T.; Qin, X.; Chen, J.; A New AIEE-Active Carbazole Based Colorimetric/Fluorimetric Chemosensor for Ultra-Rapid and Nano-Level Determination of $\mathrm{Hg}^{2+}$ and $\mathrm{Al}^{3+}$ in Food/Environmental Samples and Living Cells. Sens. Actuators B Chem. 2021, 331, 129418. (c) Zuo, Z.; Tang, Y.; Lei, F.; Jin, R.; Yin, P.; Li, Y.; Niu, Q.; New Thiophene Hydrazide Dual-Functional Chemosensor: Colorimetric Sensor for $\mathrm{Cu}^{2+} \&$ Fluorescent Sensor for $\mathrm{Al}^{3+}$ Spectrochim. Acta A Mol. Biomol. Spectrosc. 2020, 242, 118712.

16. Mohandoss, S.; Atchudan, R.; Edison, T. N. J. I.; Mishra, K.; Tamargo, R. J. I.; Palanisamy, S.; Yelithao, K.; You, S.; Lee, Y. R. Rapid Response and Highly Selective Sensing of Adenosine Based on Novel Photoluminescent Vanadium Nanoclusters Anchored on MoS2 Nanosheets. Sens. Actuators B Chem. 2020, 306, 127581. 


\section{X-ray crystallographic structure and data of compounds ( $3 \mathrm{~b}$ and $4 \mathrm{e}$ )}

The crystal structure was determined by single crystal diffraction method at the Korea Basic Science Institute (KBSI, Western Seoul Center, Korea). Colorless block crystal $\left(0.262 \times 0.187 \times 0.174 \mathrm{~mm}^{3}\right)$ was picked up with paraton oil and mounted on a Bruker D8 Venture PHOTON III M14 diffractometer equipped with a graphite-monochromated Mo $\mathrm{K} \alpha(\lambda=0.71073 \AA)$ radiation source and a nitrogen cold stream $\left(-50{ }^{\circ} \mathrm{C}\right)$. Data collection and integration were performed with SMART APEX3 (Bruker, 2016) and SAINT (Bruker, 2016) [1]. Absorption correction was performed by multi-scan method implemented in SADABS [2]. The structure was solved by direct methods and refined by full-matrix least-squares on $F^{2}$ using SHELXTL [3]. All the nonhydrogen atoms were refined anisotropically, and hydrogen atoms were added to their geometrically ideal positions.

[1] SMART, SAINT and SADABS, Bruker AXS Inc., Madison, Wisconsin, USA, 2016.

[2] G. M. Sheldrick, SADABS v 2.03, University of Göttingen, Germany, 2002.

[3] SHELXTL v 6.10; Bruker AXS, Inc: Madison, Wisconsin, USA, 2000.

\section{Method of crystal growth for compounds 3b:}

In a $5 \mathrm{~mL}$ vial, $50 \mathrm{mg}$ of the pure compound (3b) was dissolved in minimum volume of chloroform at room temperature. Then, the solution was shaken well to obtain a homogenous and clear solution. Then, the solution was covered with a porous cap and allowed to stand undisturbed at room temperature for $24 \mathrm{~h}$ until the crystals were formed via slow evaporation method.

\section{Method of crystal growth for compounds 4e:}

In a $5 \mathrm{~mL}$ vial, $75 \mathrm{mg}$ of the pure compound was dissolved in minimum volume of ethyl acetate at room temperature. Then, $1 \mathrm{~mL}$ of hexane was added to the mixture. The solution was shaken well to obtain a homogenous and clear solution. The solution was covered with a porous cap and allowed to stand undisturbed at room temperature for 2 days until the crystal was formed via slow evaporation method. 


\section{Crystal refinement data for compound $3 b$}

Empirical Formula- $\mathrm{C}_{41} \mathrm{H}_{40} \mathrm{~N}_{4} \mathrm{O}_{4}, \mathrm{M}=652.77$, Orthorhombic, Space group Pbca, a = 14.8850(3) $\AA, \mathrm{b}=13.5999(2) \AA, \mathrm{c}=43.4537(8) \AA, \mathrm{V}=8796.5(3) \AA^{3}, \mathrm{Z}=8, \mathrm{~T}=223(2)$ $\mathrm{K}, \rho$ calcd $=0.986 \mathrm{Mg} / \mathrm{m}^{3}, 2 \Theta_{\max .}=72.293^{\circ}$, Refinement of 2768 parameters on 8340 independent reflections out of 61526 collected reflections $\left(R_{i n t}=0.0479\right)$ led to $R_{1}=0$. $0791[\mathrm{I}>2 \sigma(\mathrm{I})], \mathrm{wR}_{2}=0.2414$ (all data) and $\mathrm{S}=1.059$ with the largest difference peak and hole of 0.386 and -0.395 e. $\AA^{-3}$ respectively. The crystal structure has been deposited at the Cambridge Crystallographic Data Centre (CCDC 2085983). The data can be obtained free of charge via the Internet at www.ccdc.cam.ac.uk/data_request/cif

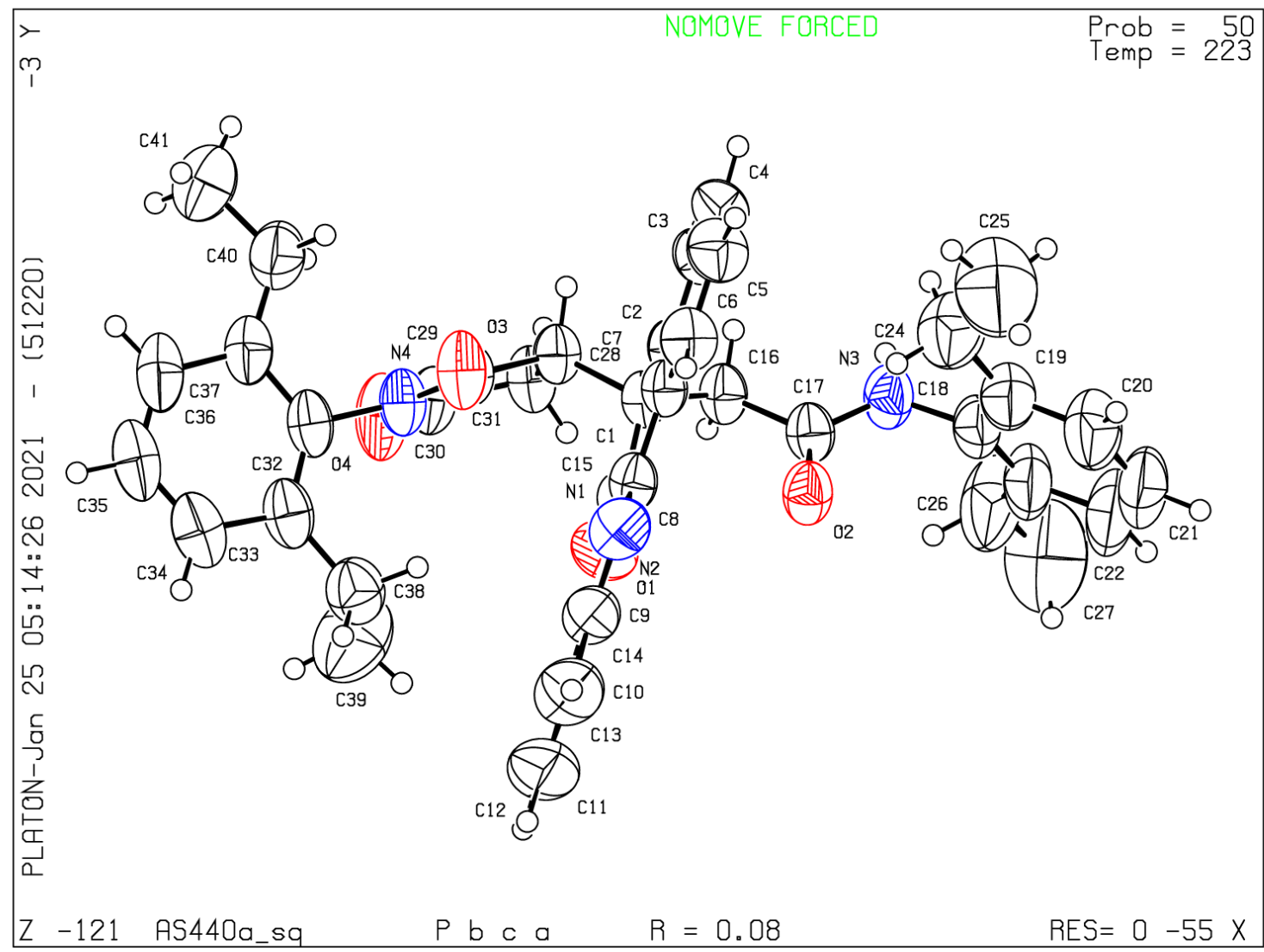

Fig. S7. X-ray structure of compound $\mathbf{3 b}$ (ellipsoid contour \% probability-50). 
Table S1. Crystal data and structure refinement for 3b (AS440a_sq).

Identification code

Empirical formula

Formula weight

Temperature

Wavelength

Crystal system

Space group

Unit cell dimensions

Volume

$\mathrm{Z}$

Density (calculated)

Absorption coefficient

$\mathrm{F}(000)$

Crystal size

Theta range for data collection

Index ranges

Reflections collected

Independent reflections

Completeness to theta $=67.679^{\circ}$

Absorption correction

Max. and min. transmission

Refinement method

Data / restraints / parameters

Goodness-of-fit on $\mathrm{F}^{2}$

Final $\mathrm{R}$ indices [I $>2 \operatorname{sigma}(\mathrm{I})]$

$\mathrm{R}$ indices (all data)

Extinction coefficient

Largest diff. peak and hole
AS440a_sq

C41 H40 N4 O4

652.77

223(2) K

$1.54178 \AA$

Orthorhombic

Pbca

$\begin{array}{ll}\mathrm{a}=14.8850(3) \AA & \alpha=90^{\circ} . \\ \mathrm{b}=13.5999(2) \AA & \beta=90^{\circ} . \\ \mathrm{c}=43.4537(8) \AA & \gamma=90^{\circ} .\end{array}$

$8796.5(3) \AA^{3}$

8

$0.986 \mathrm{Mg} / \mathrm{m}^{3}$

$0.511 \mathrm{~mm}^{-1}$

\section{8}

$0.424 \times 0.202 \times 0.175 \mathrm{~mm}^{3}$

4.520 to $72.293^{\circ}$.

$-18<=\mathrm{h}<=18,-16<=\mathrm{k}<=13,-53<=1<=52$

61526

$8340[\mathrm{R}(\mathrm{int})=0.0479]$

$96.3 \%$

Semi-empirical from equivalents

0.7536 and 0.3877

Full-matrix least-squares on $\mathrm{F}^{2}$

8340 / 0 / 446

1.059

$\mathrm{R} 1=0.0791, \mathrm{wR} 2=0.2244$

$\mathrm{R} 1=0.1009, \mathrm{wR} 2=0.2414$

$\mathrm{n} / \mathrm{a}$

0.386 and -0.395 e. $\AA^{-3}$ 
Table S2. Atomic coordinates ( $\left.\quad 10^{4}\right)$ and equivalent isotropic displacement parameters $\left(\AA^{2} \mathrm{x}\right.$ $\left.10^{3}\right)$

for AS440a_sq. $\quad U(e q)$ is defined as one third of the trace of the orthogonalized $U^{i j}$ tensor.

\begin{tabular}{|c|c|c|c|c|}
\hline & $\mathrm{x}$ & $\mathrm{y}$ & $\mathrm{z}$ & $\mathrm{U}(\mathrm{eq})$ \\
\hline $\mathrm{C}(1)$ & $7113(2)$ & $3680(2)$ & $6253(1)$ & $53(1)$ \\
\hline $\mathrm{C}(2)$ & $7366(2)$ & 4163(2) & $6557(1)$ & $53(1)$ \\
\hline$C(3)$ & $7981(2)$ & $3704(3)$ & $6753(1)$ & $63(1)$ \\
\hline $\mathrm{C}(4)$ & $8222(3)$ & 4131(3) & $7028(1)$ & $77(1)$ \\
\hline$C(5)$ & $7834(3)$ & $5013(3)$ & 7121(1) & $80(1)$ \\
\hline$C(6)$ & $7234(3)$ & $5472(3)$ & 6931(1) & $70(1)$ \\
\hline$C(7)$ & $6997(2)$ & $5057(2)$ & $6650(1)$ & $55(1)$ \\
\hline $\mathrm{C}(8)$ & $6370(2)$ & $5557(2)$ & $6450(1)$ & $58(1)$ \\
\hline $\mathrm{N}(1)$ & $6004(2)$ & $6421(2)$ & $6492(1)$ & $75(1)$ \\
\hline $\mathrm{C}(9)$ & $5447(3)$ & $6557(3)$ & $6238(1)$ & $77(1)$ \\
\hline $\mathrm{C}(10)$ & $4890(3)$ & $7350(3)$ & $6170(2)$ & $108(2)$ \\
\hline$C(11)$ & 4401(4) & $7309(4)$ & $5905(2)$ & $120(2)$ \\
\hline$C(12)$ & $4436(3)$ & $6498(5)$ & $5706(1)$ & $114(2)$ \\
\hline$C(13)$ & 4988(3) & $5695(3)$ & $5769(1)$ & $88(1)$ \\
\hline$C(14)$ & $5483(2)$ & $5757(3)$ & $6038(1)$ & $70(1)$ \\
\hline $\mathrm{N}(2)$ & 6093(2) & $5113(2)$ & $6178(1)$ & $58(1)$ \\
\hline$C(15)$ & $6379(2)$ & $4203(2)$ & $6073(1)$ & $58(1)$ \\
\hline $\mathrm{O}(1)$ & $6087(2)$ & $3876(2)$ & $5835(1)$ & $82(1)$ \\
\hline$C(16)$ & $6794(2)$ & 2611(2) & $6307(1)$ & $60(1)$ \\
\hline $\mathrm{C}(17)$ & $6003(2)$ & $2596(2)$ & $6526(1)$ & $61(1)$ \\
\hline $\mathrm{O}(2)$ & $5478(2)$ & $3293(2)$ & $6539(1)$ & $70(1)$ \\
\hline $\mathrm{N}(3)$ & $5923(2)$ & $1786(2)$ & $6698(1)$ & $76(1)$ \\
\hline $\mathrm{C}(18)$ & $5203(3)$ & $1647(3)$ & 6911(1) & $77(1)$ \\
\hline$C(19)$ & $5245(3)$ & $2137(3)$ & 7194(1) & $88(1)$ \\
\hline $\mathrm{C}(20)$ & $4518(4)$ & 2010(4) & 7392(1) & $113(2)$ \\
\hline$C(21)$ & $3811(4)$ & $1409(5)$ & 7311(1) & $133(2)$ \\
\hline $\mathrm{C}(22)$ & $3818(4)$ & $916(6)$ & $7036(1)$ & $145(3)$ \\
\hline$C(23)$ & $4518(3)$ & 1004(4) & 6831(1) & $107(2)$ \\
\hline$C(24)$ & $6051(4)$ & 2761(4) & $7273(1)$ & $112(2)$ \\
\hline$C(25)$ & $6136(6)$ & $3100(7)$ & $7596(2)$ & $185(4)$ \\
\hline$C(26)$ & $4580(5)$ & $413(6)$ & $6530(1)$ & $161(3)$ \\
\hline
\end{tabular}




$\begin{array}{lrrrr}\mathrm{C}(27) & 3901(6) & -279(5) & 6455(2) & 183(3) \\ \mathrm{C}(28) & 7960(2) & 3650(2) & 6039(1) & 58(1) \\ \mathrm{C}(29) & 8193(2) & 4672(2) & 5916(1) & 60(1) \\ \mathrm{N}(4) & 8349(2) & 4599(2) & 5604(1) & 64(1) \\ \mathrm{C}(30) & 8271(2) & 3630(3) & 5497(1) & 69(1) \\ \mathrm{C}(31) & 7940(3) & 2998(2) & 5753(1) & 65(1) \\ \mathrm{O}(3) & 8232(2) & 5429(2) & 6061(1) & 78(1) \\ \mathrm{O}(4) & 8429(2) & 3390(2) & 5234(1) & 95(1) \\ \mathrm{C}(32) & 8627(3) & 5414(3) & 5415(1) & 68(1) \\ \mathrm{C}(33) & 7976(3) & 5968(3) & 5268(1) & 75(1) \\ \mathrm{C}(34) & 8278(4) & 6781(3) & 5096(1) & 89(1) \\ \mathrm{C}(35) & 9173(4) & 6991(3) & 5074(1) & 96(1) \\ \mathrm{C}(36) & 9798(4) & 6412(4) & 5217(1) & 95(1) \\ \mathrm{C}(37) & 9550(3) & 5604(3) & 5398(1) & 77(1) \\ \mathrm{C}(38) & 6993(3) & 5732(3) & 5284(1) & 88(1) \\ \mathrm{C}(39) & 6666(4) & 5086(6) & 5032(2) & 150(2) \\ \mathrm{C}(40) & 10212(3) & 4946(4) & 5557(1) & 96(1) \\ \mathrm{C}(41) & 11187(3) & 5122(4) & 5496(1) & 117(2)\end{array}$

Table S3. Bond lengths $[\AA]$ and angles $\left[{ }^{\circ}\right]$ for $\quad$ AS440a_sq.

\begin{tabular}{ll}
\hline $\mathrm{C}(1)-\mathrm{C}(15)$ & $1.520(4)$ \\
$\mathrm{C}(1)-\mathrm{C}(2)$ & $1.523(4)$ \\
$\mathrm{C}(1)-\mathrm{C}(16)$ & $1.549(4)$ \\
$\mathrm{C}(1)-\mathrm{C}(28)$ & $1.566(4)$ \\
$\mathrm{C}(2)-\mathrm{C}(7)$ & $1.394(4)$ \\
$\mathrm{C}(2)-\mathrm{C}(3)$ & $1.399(4)$ \\
$\mathrm{C}(3)-\mathrm{C}(4)$ & $1.374(5)$ \\
$\mathrm{C}(3)-\mathrm{H}(3)$ & 0.9400 \\
$\mathrm{C}(4)-\mathrm{C}(5)$ & $1.390(5)$ \\
$\mathrm{C}(4)-\mathrm{H}(4)$ & 0.9400 \\
$\mathrm{C}(5)-\mathrm{C}(6)$ & $1.367(5)$ \\
$\mathrm{C}(5)-\mathrm{H}(5)$ & 0.9400 \\
$\mathrm{C}(6)-\mathrm{C}(7)$ & $1.392(4)$ \\
$\mathrm{C}(6)-\mathrm{H}(6)$ & 0.9400 \\
$\mathrm{C}(7)-\mathrm{C}(8)$ & $1.445(5)$
\end{tabular}




\begin{tabular}{|c|c|}
\hline $\mathrm{C}(8)-\mathrm{N}(1)$ & $1.307(4)$ \\
\hline $\mathrm{C}(8)-\mathrm{N}(2)$ & $1.389(4)$ \\
\hline $\mathrm{N}(1)-\mathrm{C}(9)$ & $1.394(5)$ \\
\hline$C(9)-C(10)$ & $1.391(6)$ \\
\hline$C(9)-C(14)$ & $1.395(6)$ \\
\hline $\mathrm{C}(10)-\mathrm{C}(11)$ & $1.364(8)$ \\
\hline $\mathrm{C}(10)-\mathrm{H}(10)$ & 0.9400 \\
\hline $\mathrm{C}(11)-\mathrm{C}(12)$ & $1.402(8)$ \\
\hline $\mathrm{C}(11)-\mathrm{H}(11)$ & 0.9400 \\
\hline $\mathrm{C}(12)-\mathrm{C}(13)$ & $1.393(7)$ \\
\hline $\mathrm{C}(12)-\mathrm{H}(12)$ & 0.9400 \\
\hline $\mathrm{C}(13)-\mathrm{C}(14)$ & $1.381(6)$ \\
\hline $\mathrm{C}(13)-\mathrm{H}(13)$ & 0.9400 \\
\hline $\mathrm{C}(14)-\mathrm{N}(2)$ & $1.401(4)$ \\
\hline $\mathrm{N}(2)-\mathrm{C}(15)$ & $1.387(4)$ \\
\hline $\mathrm{C}(15)-\mathrm{O}(1)$ & $1.206(4)$ \\
\hline $\mathrm{C}(16)-\mathrm{C}(17)$ & $1.512(4)$ \\
\hline $\mathrm{C}(16)-\mathrm{H}(16 \mathrm{~A})$ & 0.9800 \\
\hline $\mathrm{C}(16)-\mathrm{H}(16 \mathrm{~B})$ & 0.9800 \\
\hline $\mathrm{C}(17)-\mathrm{O}(2)$ & $1.229(4)$ \\
\hline $\mathrm{C}(17)-\mathrm{N}(3)$ & $1.338(4)$ \\
\hline $\mathrm{N}(3)-\mathrm{C}(18)$ & $1.430(5)$ \\
\hline $\mathrm{N}(3)-\mathrm{H}(3 \mathrm{~A})$ & 0.8700 \\
\hline $\mathrm{C}(18)-\mathrm{C}(23)$ & $1.389(6)$ \\
\hline $\mathrm{C}(18)-\mathrm{C}(19)$ & $1.396(6)$ \\
\hline$C(19)-C(20)$ & $1.393(6)$ \\
\hline $\mathrm{C}(19)-\mathrm{C}(24)$ & $1.509(6)$ \\
\hline$C(20)-C(21)$ & $1.379(8)$ \\
\hline $\mathrm{C}(20)-\mathrm{H}(20)$ & 0.9400 \\
\hline $\mathrm{C}(21)-\mathrm{C}(22)$ & $1.366(8)$ \\
\hline $\mathrm{C}(21)-\mathrm{H}(21)$ & 0.9400 \\
\hline$C(22)-C(23)$ & $1.377(7)$ \\
\hline $\mathrm{C}(22)-\mathrm{H}(22)$ & 0.9400 \\
\hline$C(23)-C(26)$ & $1.539(8)$ \\
\hline $\mathrm{C}(24)-\mathrm{C}(25)$ & $1.484(8)$ \\
\hline $\mathrm{C}(24)-\mathrm{H}(24 \mathrm{~A})$ & 0.9800 \\
\hline $\mathrm{C}(24)-\mathrm{H}(24 \mathrm{~B})$ & 0.9800 \\
\hline
\end{tabular}




\begin{tabular}{|c|c|}
\hline $\mathrm{C}(25)-\mathrm{H}(25 \mathrm{~A})$ & 0.9700 \\
\hline $\mathrm{C}(25)-\mathrm{H}(25 \mathrm{~B})$ & 0.9700 \\
\hline $\mathrm{C}(25)-\mathrm{H}(25 \mathrm{C})$ & 0.9700 \\
\hline$C(26)-C(27)$ & $1.418(9)$ \\
\hline $\mathrm{C}(26)-\mathrm{H}(26 \mathrm{~A})$ & 0.9800 \\
\hline $\mathrm{C}(26)-\mathrm{H}(26 \mathrm{~B})$ & 0.9800 \\
\hline $\mathrm{C}(27)-\mathrm{H}(27 \mathrm{~A})$ & 0.9700 \\
\hline $\mathrm{C}(27)-\mathrm{H}(27 \mathrm{~B})$ & 0.9700 \\
\hline $\mathrm{C}(27)-\mathrm{H}(27 \mathrm{C})$ & 0.9700 \\
\hline $\mathrm{C}(28)-\mathrm{C}(31)$ & $1.529(4)$ \\
\hline$C(28)-C(29)$ & $1.530(4)$ \\
\hline $\mathrm{C}(28)-\mathrm{H}(28)$ & 0.9900 \\
\hline $\mathrm{C}(29)-\mathrm{O}(3)$ & $1.209(4)$ \\
\hline $\mathrm{C}(29)-\mathrm{N}(4)$ & $1.376(4)$ \\
\hline $\mathrm{N}(4)-\mathrm{C}(30)$ & $1.402(4)$ \\
\hline $\mathrm{N}(4)-\mathrm{C}(32)$ & $1.442(4)$ \\
\hline $\mathrm{C}(30)-\mathrm{O}(4)$ & $1.210(4)$ \\
\hline $\mathrm{C}(30)-\mathrm{C}(31)$ & $1.490(5)$ \\
\hline $\mathrm{C}(31)-\mathrm{H}(31 \mathrm{~A})$ & 0.9800 \\
\hline $\mathrm{C}(31)-\mathrm{H}(31 \mathrm{~B})$ & 0.9800 \\
\hline$C(32)-C(33)$ & $1.383(5)$ \\
\hline$C(32)-C(37)$ & $1.400(5)$ \\
\hline $\mathrm{C}(33)-\mathrm{C}(34)$ & $1.408(5)$ \\
\hline$C(33)-C(38)$ & $1.499(6)$ \\
\hline $\mathrm{C}(34)-\mathrm{C}(35)$ & $1.367(7)$ \\
\hline $\mathrm{C}(34)-\mathrm{H}(34)$ & 0.9400 \\
\hline$C(35)-C(36)$ & $1.369(7)$ \\
\hline $\mathrm{C}(35)-\mathrm{H}(35)$ & 0.9400 \\
\hline$C(36)-C(37)$ & $1.399(5)$ \\
\hline $\mathrm{C}(36)-\mathrm{H}(36)$ & 0.9400 \\
\hline $\mathrm{C}(37)-\mathrm{C}(40)$ & $1.500(6)$ \\
\hline $\mathrm{C}(38)-\mathrm{C}(39)$ & $1.485(7)$ \\
\hline $\mathrm{C}(38)-\mathrm{H}(38 \mathrm{~A})$ & 0.9800 \\
\hline $\mathrm{C}(38)-\mathrm{H}(38 \mathrm{~B})$ & 0.9800 \\
\hline $\mathrm{C}(39)-\mathrm{H}(39 \mathrm{~A})$ & 0.9700 \\
\hline $\mathrm{C}(39)-\mathrm{H}(39 \mathrm{~B})$ & 0.9700 \\
\hline C(39)-H(39C) & 0.9700 \\
\hline
\end{tabular}




\begin{tabular}{|c|c|}
\hline$C(40)-C(41)$ & $1.494(6)$ \\
\hline $\mathrm{C}(40)-\mathrm{H}(40 \mathrm{~A})$ & 0.9800 \\
\hline $\mathrm{C}(40)-\mathrm{H}(40 \mathrm{~B})$ & 0.9800 \\
\hline $\mathrm{C}(41)-\mathrm{H}(41 \mathrm{~A})$ & 0.9700 \\
\hline $\mathrm{C}(41)-\mathrm{H}(41 \mathrm{~B})$ & 0.9700 \\
\hline $\mathrm{C}(41)-\mathrm{H}(41 \mathrm{C})$ & 0.9700 \\
\hline $\mathrm{C}(15)-\mathrm{C}(1)-\mathrm{C}(2)$ & $115.1(2)$ \\
\hline$C(15)-C(1)-C(16)$ & 107.3(3) \\
\hline $\mathrm{C}(2)-\mathrm{C}(1)-\mathrm{C}(16)$ & $110.4(2)$ \\
\hline $\mathrm{C}(15)-\mathrm{C}(1)-\mathrm{C}(28)$ & $106.6(2)$ \\
\hline $\mathrm{C}(2)-\mathrm{C}(1)-\mathrm{C}(28)$ & $109.0(2)$ \\
\hline$C(16)-C(1)-C(28)$ & $108.2(2)$ \\
\hline $\mathrm{C}(7)-\mathrm{C}(2)-\mathrm{C}(3)$ & 118.1(3) \\
\hline$C(7)-C(2)-C(1)$ & $122.0(3)$ \\
\hline $\mathrm{C}(3)-\mathrm{C}(2)-\mathrm{C}(1)$ & $120.0(3)$ \\
\hline$C(4)-C(3)-C(2)$ & $120.8(3)$ \\
\hline $\mathrm{C}(4)-\mathrm{C}(3)-\mathrm{H}(3)$ & 119.6 \\
\hline $\mathrm{C}(2)-\mathrm{C}(3)-\mathrm{H}(3)$ & 119.6 \\
\hline$C(3)-C(4)-C(5)$ & $120.6(4)$ \\
\hline $\mathrm{C}(3)-\mathrm{C}(4)-\mathrm{H}(4)$ & 119.7 \\
\hline $\mathrm{C}(5)-\mathrm{C}(4)-\mathrm{H}(4)$ & 119.7 \\
\hline$C(6)-C(5)-C(4)$ & 119.3(3) \\
\hline $\mathrm{C}(6)-\mathrm{C}(5)-\mathrm{H}(5)$ & 120.3 \\
\hline $\mathrm{C}(4)-\mathrm{C}(5)-\mathrm{H}(5)$ & 120.3 \\
\hline$C(5)-C(6)-C(7)$ & $120.7(3)$ \\
\hline $\mathrm{C}(5)-\mathrm{C}(6)-\mathrm{H}(6)$ & 119.7 \\
\hline $\mathrm{C}(7)-\mathrm{C}(6)-\mathrm{H}(6)$ & 119.7 \\
\hline$C(6)-C(7)-C(2)$ & $120.5(3)$ \\
\hline$C(6)-C(7)-C(8)$ & $120.0(3)$ \\
\hline$C(2)-C(7)-C(8)$ & $119.5(3)$ \\
\hline $\mathrm{N}(1)-\mathrm{C}(8)-\mathrm{N}(2)$ & $112.7(3)$ \\
\hline $\mathrm{N}(1)-\mathrm{C}(8)-\mathrm{C}(7)$ & $127.5(3)$ \\
\hline $\mathrm{N}(2)-\mathrm{C}(8)-\mathrm{C}(7)$ & 119.8(3) \\
\hline $\mathrm{C}(8)-\mathrm{N}(1)-\mathrm{C}(9)$ & $104.9(3)$ \\
\hline $\mathrm{C}(10)-\mathrm{C}(9)-\mathrm{N}(1)$ & $128.7(4)$ \\
\hline$C(10)-C(9)-C(14)$ & $119.8(4)$ \\
\hline $\mathrm{N}(1)-\mathrm{C}(9)-\mathrm{C}(14)$ & $111.5(3)$ \\
\hline
\end{tabular}




$\begin{array}{ll}\mathrm{C}(11)-\mathrm{C}(10)-\mathrm{C}(9) & 117.7(5) \\ \mathrm{C}(11)-\mathrm{C}(10)-\mathrm{H}(10) & 121.2 \\ \mathrm{C}(9)-\mathrm{C}(10)-\mathrm{H}(10) & 121.2 \\ \mathrm{C}(10)-\mathrm{C}(11)-\mathrm{C}(12) & 122.2(5) \\ \mathrm{C}(10)-\mathrm{C}(11)-\mathrm{H}(11) & 118.9 \\ \mathrm{C}(12)-\mathrm{C}(11)-\mathrm{H}(11) & 118.9 \\ \mathrm{C}(13)-\mathrm{C}(12)-\mathrm{C}(11) & 121.2(5) \\ \mathrm{C}(13)-\mathrm{C}(12)-\mathrm{H}(12) & 119.4 \\ \mathrm{C}(11)-\mathrm{C}(12)-\mathrm{H}(12) & 119.4 \\ \mathrm{C}(14)-\mathrm{C}(13)-\mathrm{C}(12) & 115.7(5) \\ \mathrm{C}(14)-\mathrm{C}(13)-\mathrm{H}(13) & 122.2 \\ \mathrm{C}(12)-\mathrm{C}(13)-\mathrm{H}(13) & 122.2 \\ \mathrm{C}(13)-\mathrm{C}(14)-\mathrm{C}(9) & 123.6(4) \\ \mathrm{C}(13)-\mathrm{C}(14)-\mathrm{N}(2) & 132.5(4) \\ \mathrm{C}(9)-\mathrm{C}(14)-\mathrm{N}(2) & 103.9(3) \\ \mathrm{C}(15)-\mathrm{N}(2)-\mathrm{C}(8) & 125.3(3) \\ \mathrm{C}(15)-\mathrm{N}(2)-\mathrm{C}(14) & 127.7(3) \\ \mathrm{C}(8)-\mathrm{N}(2)-\mathrm{C}(14) & 106.9(3) \\ \mathrm{O}(1)-\mathrm{C}(15)-\mathrm{N}(2) & 120.1(3) \\ \mathrm{O}(1)-\mathrm{C}(15)-\mathrm{C}(1) & 121.9(3) \\ \mathrm{N}(2)-\mathrm{C}(15)-\mathrm{C}(1) & 117.8(3) \\ \mathrm{C}(17)-\mathrm{C}(16)-\mathrm{C}(1) & 110.3(2) \\ \mathrm{C}(17)-\mathrm{C}(16)-\mathrm{H}(16 \mathrm{~A}) & 109.6 \\ \mathrm{C}(1)-\mathrm{C}(16)-\mathrm{H}(16 \mathrm{~A}) & 109.6 \\ \mathrm{C}(17)-\mathrm{C}(16)-\mathrm{H}(16 \mathrm{~B}) & 109.6 \\ \mathrm{C}(1)-\mathrm{C}(16)-\mathrm{H}(16 \mathrm{~B}) & 109.6 \\ \mathrm{H}(16 \mathrm{~A})-\mathrm{C}(16)-\mathrm{H}(16 \mathrm{~B}) & 108.1 \\ \mathrm{O}(2)-\mathrm{C}(17)-\mathrm{N}(3) & 123.4(3) \\ \mathrm{O}(2)-\mathrm{C}(17)-\mathrm{C}(16) & 121.0(3) \\ \mathrm{N}(3)-\mathrm{C}(17)-\mathrm{C}(16) & 115.6(3) \\ \mathrm{C}(17)-\mathrm{N}(3)-\mathrm{C}(18) & 122.6(3) \\ \mathrm{C}(17)-\mathrm{N}(3)-\mathrm{H}(3 \mathrm{~A}) & 118.7 \\ \mathrm{C}(18)-\mathrm{N}(3)-\mathrm{H}(3 \mathrm{~A}) & 118.7 \\ \mathrm{C}(23)-\mathrm{C}(18)-\mathrm{C}(19) & 123.7(4) \\ \mathrm{C}(19)-\mathrm{C}(18) & 118.0(4) \\ & \\ \mathrm{C}(3) & \\ \mathrm{C}(1) .6(4) \\ \end{array}$




\begin{tabular}{|c|c|}
\hline$C(20)-C(19)-C(24)$ & $123.0(4)$ \\
\hline $\mathrm{C}(18)-\mathrm{C}(19)-\mathrm{C}(24)$ & $120.4(4)$ \\
\hline$C(21)-C(20)-C(19)$ & $120.6(5)$ \\
\hline $\mathrm{C}(21)-\mathrm{C}(20)-\mathrm{H}(20)$ & 119.7 \\
\hline $\mathrm{C}(19)-\mathrm{C}(20)-\mathrm{H}(20)$ & 119.7 \\
\hline$C(22)-C(21)-C(20)$ & $120.5(5)$ \\
\hline $\mathrm{C}(22)-\mathrm{C}(21)-\mathrm{H}(21)$ & 119.8 \\
\hline $\mathrm{C}(20)-\mathrm{C}(21)-\mathrm{H}(21)$ & 119.8 \\
\hline$C(21)-C(22)-C(23)$ & $121.9(5)$ \\
\hline $\mathrm{C}(21)-\mathrm{C}(22)-\mathrm{H}(22)$ & 119.0 \\
\hline $\mathrm{C}(23)-\mathrm{C}(22)-\mathrm{H}(22)$ & 119.0 \\
\hline$C(22)-C(23)-C(18)$ & $116.5(5)$ \\
\hline$C(22)-C(23)-C(26)$ & $123.5(5)$ \\
\hline $\mathrm{C}(18)-\mathrm{C}(23)-\mathrm{C}(26)$ & $120.0(4)$ \\
\hline$C(25)-C(24)-C(19)$ & $117.4(5)$ \\
\hline $\mathrm{C}(25)-\mathrm{C}(24)-\mathrm{H}(24 \mathrm{~A})$ & 108.0 \\
\hline $\mathrm{C}(19)-\mathrm{C}(24)-\mathrm{H}(24 \mathrm{~A})$ & 108.0 \\
\hline $\mathrm{C}(25)-\mathrm{C}(24)-\mathrm{H}(24 \mathrm{~B})$ & 108.0 \\
\hline $\mathrm{C}(19)-\mathrm{C}(24)-\mathrm{H}(24 \mathrm{~B})$ & 108.0 \\
\hline $\mathrm{H}(24 \mathrm{~A})-\mathrm{C}(24)-\mathrm{H}(24 \mathrm{~B})$ & 107.2 \\
\hline $\mathrm{C}(24)-\mathrm{C}(25)-\mathrm{H}(25 \mathrm{~A})$ & 109.5 \\
\hline $\mathrm{C}(24)-\mathrm{C}(25)-\mathrm{H}(25 \mathrm{~B})$ & 109.5 \\
\hline $\mathrm{H}(25 \mathrm{~A})-\mathrm{C}(25)-\mathrm{H}(25 \mathrm{~B})$ & 109.5 \\
\hline $\mathrm{C}(24)-\mathrm{C}(25)-\mathrm{H}(25 \mathrm{C})$ & 109.5 \\
\hline $\mathrm{H}(25 \mathrm{~A})-\mathrm{C}(25)-\mathrm{H}(25 \mathrm{C})$ & 109.5 \\
\hline$H(25 B)-C(25)-H(25 C)$ & 109.5 \\
\hline$C(27)-C(26)-C(23)$ & $119.8(5)$ \\
\hline $\mathrm{C}(27)-\mathrm{C}(26)-\mathrm{H}(26 \mathrm{~A})$ & 107.4 \\
\hline $\mathrm{C}(23)-\mathrm{C}(26)-\mathrm{H}(26 \mathrm{~A})$ & 107.4 \\
\hline $\mathrm{C}(27)-\mathrm{C}(26)-\mathrm{H}(26 \mathrm{~B})$ & 107.4 \\
\hline $\mathrm{C}(23)-\mathrm{C}(26)-\mathrm{H}(26 \mathrm{~B})$ & 107.4 \\
\hline$H(26 A)-C(26)-H(26 B)$ & 106.9 \\
\hline $\mathrm{C}(26)-\mathrm{C}(27)-\mathrm{H}(27 \mathrm{~A})$ & 109.5 \\
\hline $\mathrm{C}(26)-\mathrm{C}(27)-\mathrm{H}(27 \mathrm{~B})$ & 109.5 \\
\hline $\mathrm{H}(27 \mathrm{~A})-\mathrm{C}(27)-\mathrm{H}(27 \mathrm{~B})$ & 109.5 \\
\hline $\mathrm{C}(26)-\mathrm{C}(27)-\mathrm{H}(27 \mathrm{C})$ & 109.5 \\
\hline $\mathrm{H}(27 \mathrm{~A})-\mathrm{C}(27)-\mathrm{H}(27 \mathrm{C})$ & 109.5 \\
\hline
\end{tabular}




\begin{tabular}{|c|c|}
\hline$H(27 B)-C(27)-H(27 C)$ & 109.5 \\
\hline$C(31)-C(28)-C(29)$ & $104.2(2)$ \\
\hline $\mathrm{C}(31)-\mathrm{C}(28)-\mathrm{C}(1)$ & 118.7(3) \\
\hline$C(29)-C(28)-C(1)$ & $111.5(2)$ \\
\hline $\mathrm{C}(31)-\mathrm{C}(28)-\mathrm{H}(28)$ & 107.3 \\
\hline $\mathrm{C}(29)-\mathrm{C}(28)-\mathrm{H}(28)$ & 107.3 \\
\hline $\mathrm{C}(1)-\mathrm{C}(28)-\mathrm{H}(28)$ & 107.3 \\
\hline $\mathrm{O}(3)-\mathrm{C}(29)-\mathrm{N}(4)$ & $124.5(3)$ \\
\hline $\mathrm{O}(3)-\mathrm{C}(29)-\mathrm{C}(28)$ & $126.9(3)$ \\
\hline $\mathrm{N}(4)-\mathrm{C}(29)-\mathrm{C}(28)$ & $108.5(3)$ \\
\hline $\mathrm{C}(29)-\mathrm{N}(4)-\mathrm{C}(30)$ & $112.4(3)$ \\
\hline$C(29)-N(4)-C(32)$ & $123.6(3)$ \\
\hline $\mathrm{C}(30)-\mathrm{N}(4)-\mathrm{C}(32)$ & $123.8(2)$ \\
\hline $\mathrm{O}(4)-\mathrm{C}(30)-\mathrm{N}(4)$ & $123.4(3)$ \\
\hline $\mathrm{O}(4)-\mathrm{C}(30)-\mathrm{C}(31)$ & $127.8(3)$ \\
\hline $\mathrm{N}(4)-\mathrm{C}(30)-\mathrm{C}(31)$ & $108.7(2)$ \\
\hline $\mathrm{C}(30)-\mathrm{C}(31)-\mathrm{C}(28)$ & $105.4(3)$ \\
\hline $\mathrm{C}(30)-\mathrm{C}(31)-\mathrm{H}(31 \mathrm{~A})$ & 110.7 \\
\hline $\mathrm{C}(28)-\mathrm{C}(31)-\mathrm{H}(31 \mathrm{~A})$ & 110.7 \\
\hline $\mathrm{C}(30)-\mathrm{C}(31)-\mathrm{H}(31 \mathrm{~B})$ & 110.7 \\
\hline $\mathrm{C}(28)-\mathrm{C}(31)-\mathrm{H}(31 \mathrm{~B})$ & 110.7 \\
\hline $\mathrm{H}(31 \mathrm{~A})-\mathrm{C}(31)-\mathrm{H}(31 \mathrm{~B})$ & 108.8 \\
\hline $\mathrm{C}(33)-\mathrm{C}(32)-\mathrm{C}(37)$ & $124.3(3)$ \\
\hline $\mathrm{C}(33)-\mathrm{C}(32)-\mathrm{N}(4)$ & $118.8(3)$ \\
\hline $\mathrm{C}(37)-\mathrm{C}(32)-\mathrm{N}(4)$ & $116.9(3)$ \\
\hline $\mathrm{C}(32)-\mathrm{C}(33)-\mathrm{C}(34)$ & $116.6(4)$ \\
\hline $\mathrm{C}(32)-\mathrm{C}(33)-\mathrm{C}(38)$ & 123.1(3) \\
\hline $\mathrm{C}(34)-\mathrm{C}(33)-\mathrm{C}(38)$ & $120.3(4)$ \\
\hline$C(35)-C(34)-C(33)$ & $120.8(4)$ \\
\hline $\mathrm{C}(35)-\mathrm{C}(34)-\mathrm{H}(34)$ & 119.6 \\
\hline $\mathrm{C}(33)-\mathrm{C}(34)-\mathrm{H}(34)$ & 119.6 \\
\hline $\mathrm{C}(34)-\mathrm{C}(35)-\mathrm{C}(36)$ & $120.7(4)$ \\
\hline $\mathrm{C}(34)-\mathrm{C}(35)-\mathrm{H}(35)$ & 119.6 \\
\hline $\mathrm{C}(36)-\mathrm{C}(35)-\mathrm{H}(35)$ & 119.6 \\
\hline$C(35)-C(36)-C(37)$ & $121.8(4)$ \\
\hline $\mathrm{C}(35)-\mathrm{C}(36)-\mathrm{H}(36)$ & 119.1 \\
\hline $\mathrm{C}(37)-\mathrm{C}(36)-\mathrm{H}(36)$ & 119.1 \\
\hline
\end{tabular}




$\begin{array}{ll}\mathrm{C}(36)-\mathrm{C}(37)-\mathrm{C}(32) & 115.7(4) \\ \mathrm{C}(36)-\mathrm{C}(37)-\mathrm{C}(40) & 123.6(4) \\ \mathrm{C}(32)-\mathrm{C}(37)-\mathrm{C}(40) & 120.7(3) \\ \mathrm{C}(39)-\mathrm{C}(38)-\mathrm{C}(33) & 114.3(4) \\ \mathrm{C}(39)-\mathrm{C}(38)-\mathrm{H}(38 \mathrm{~A}) & 108.7 \\ \mathrm{C}(33)-\mathrm{C}(38)-\mathrm{H}(38 \mathrm{~A}) & 108.7 \\ \mathrm{C}(39)-\mathrm{C}(38)-\mathrm{H}(38 \mathrm{~B}) & 108.7 \\ \mathrm{C}(33)-\mathrm{C}(38)-\mathrm{H}(38 \mathrm{~B}) & 108.7 \\ \mathrm{H}(38 \mathrm{~A})-\mathrm{C}(38)-\mathrm{H}(38 \mathrm{~B}) & 107.6 \\ \mathrm{C}(38)-\mathrm{C}(39)-\mathrm{H}(39 \mathrm{~A}) & 109.5 \\ \mathrm{C}(38)-\mathrm{C}(39)-\mathrm{H}(39 \mathrm{~B}) & 109.5 \\ \mathrm{H}(39 \mathrm{~A})-\mathrm{C}(39)-\mathrm{H}(39 \mathrm{~B}) & 109.5 \\ \mathrm{C}(38)-\mathrm{C}(39)-\mathrm{H}(39 \mathrm{C}) & 109.5 \\ \mathrm{H}(39 \mathrm{~A})-\mathrm{C}(39)-\mathrm{H}(39 \mathrm{C}) & 109.5 \\ \mathrm{H}(39 \mathrm{~B})-\mathrm{C}(39)-\mathrm{H}(39 \mathrm{C}) & 109.5 \\ \mathrm{C}(41)-\mathrm{C}(40)-\mathrm{C}(37) & 117.4(4) \\ \mathrm{C}(41)-\mathrm{C}(40)-\mathrm{H}(40 \mathrm{~A}) & 107.9 \\ \mathrm{C}(37)-\mathrm{C}(40)-\mathrm{H}(40 \mathrm{~A}) & 107.9 \\ \mathrm{C}(41)-\mathrm{C}(40)-\mathrm{H}(40 \mathrm{~B}) & 107.9 \\ \mathrm{C}(37)-\mathrm{C}(40)-\mathrm{H}(40 \mathrm{~B}) & 107.9 \\ \mathrm{H}(40 \mathrm{~A})-\mathrm{C}(40)-\mathrm{H}(40 \mathrm{~B}) & 107.2 \\ \mathrm{C}(40)-\mathrm{C}(41)-\mathrm{H}(41 \mathrm{~A}) & 109.5 \\ \mathrm{C}(40)-\mathrm{C}(41)-\mathrm{H}(41 \mathrm{~B}) & 109.5 \\ \mathrm{H}(41 \mathrm{~A})-\mathrm{C}(41)-\mathrm{H}(41 \mathrm{~B}) & 109.5 \\ \mathrm{C}(40)-\mathrm{C}(41)-\mathrm{H}(41 \mathrm{C}) & 109.5 \\ \mathrm{H}(41 \mathrm{~A})-\mathrm{C}(41)-\mathrm{H}(41 \mathrm{C}) & 109.5 \\ \mathrm{H}(41 \mathrm{~B})-\mathrm{C}(41)-\mathrm{H}(41 \mathrm{C}) & 109.5 \\ & \end{array}$

Symmetry transformations used to generate equivalent atoms: 
Table S4. Anisotropic displacement parameters $\left(\AA^{2} \mathrm{x} 10^{3}\right)$ for AS440a_sq. The anisotropic displacement factor exponent takes the form: $-2 \pi^{2}\left[h^{2} a^{* 2} U^{11}+\ldots \quad+2 h k\right.$ a* $\left.b^{*} U^{12}\right]$

\begin{tabular}{|c|c|c|c|c|c|c|}
\hline & $\mathrm{U}^{11}$ & $\mathrm{U}^{22}$ & $\mathrm{U}^{33}$ & $\mathrm{U}^{23}$ & $\mathrm{U}^{13}$ & $\mathrm{U}^{12}$ \\
\hline $\mathrm{C}(1)$ & $67(2)$ & $40(2)$ & $51(1)$ & $-2(1)$ & $13(1)$ & $0(1)$ \\
\hline$C(2)$ & $64(2)$ & $43(2)$ & $53(1)$ & $2(1)$ & $13(1)$ & $-3(1)$ \\
\hline$C(3)$ & $76(2)$ & $56(2)$ & $58(2)$ & $5(2)$ & $6(2)$ & $6(2)$ \\
\hline $\mathrm{C}(4)$ & $85(2)$ & $82(3)$ & $63(2)$ & $7(2)$ & $-4(2)$ & $2(2)$ \\
\hline$C(5)$ & $96(3)$ & $87(3)$ & $57(2)$ & $-14(2)$ & $0(2)$ & $-4(2)$ \\
\hline$C(6)$ & $86(2)$ & $62(2)$ & $60(2)$ & $-16(2)$ & $9(2)$ & $-2(2)$ \\
\hline$C(7)$ & $66(2)$ & $44(2)$ & $55(2)$ & $-2(1)$ & 11(1) & $-3(1)$ \\
\hline $\mathrm{C}(8)$ & $68(2)$ & $38(2)$ & $67(2)$ & $-3(1)$ & $11(2)$ & $-4(1)$ \\
\hline $\mathrm{N}(1)$ & $77(2)$ & $44(2)$ & $105(2)$ & $-8(2)$ & $7(2)$ & $4(1)$ \\
\hline $\mathrm{C}(9)$ & $70(2)$ & $54(2)$ & $108(3)$ & $11(2)$ & $6(2)$ & $6(2)$ \\
\hline$C(10)$ & $93(3)$ & $66(3)$ & $165(5)$ & $18(3)$ & $7(3)$ & $14(2)$ \\
\hline $\mathrm{C}(11)$ & $96(3)$ & $98(4)$ & $165(5)$ & $42(4)$ & $-6(4)$ & $26(3)$ \\
\hline$C(12)$ & $90(3)$ & $131(5)$ & $120(4)$ & $55(4)$ & $-10(3)$ & $17(3)$ \\
\hline$C(13)$ & $85(3)$ & $97(3)$ & $82(2)$ & $27(2)$ & $0(2)$ & $7(2)$ \\
\hline$C(14)$ & $66(2)$ & $58(2)$ & $84(2)$ & $24(2)$ & $8(2)$ & $4(2)$ \\
\hline $\mathrm{N}(2)$ & $69(2)$ & $44(1)$ & $63(1)$ & $7(1)$ & $7(1)$ & $2(1)$ \\
\hline$C(15)$ & $72(2)$ & $47(2)$ & $57(2)$ & $-1(1)$ & $8(1)$ & $-1(1)$ \\
\hline $\mathrm{O}(1)$ & $98(2)$ & $81(2)$ & $65(1)$ & $-14(1)$ & $-13(1)$ & $5(1)$ \\
\hline$C(16)$ & $74(2)$ & $44(2)$ & $63(2)$ & $-1(1)$ & $16(2)$ & $0(1)$ \\
\hline $\mathrm{C}(17)$ & $75(2)$ & $46(2)$ & $61(2)$ & $-1(1)$ & $10(2)$ & $-3(2)$ \\
\hline $\mathrm{O}(2)$ & $79(2)$ & $54(1)$ & $78(1)$ & $5(1)$ & $23(1)$ & $4(1)$ \\
\hline $\mathrm{N}(3)$ & $81(2)$ & $54(2)$ & $92(2)$ & $15(2)$ & $18(2)$ & $-3(1)$ \\
\hline $\mathrm{C}(18)$ & $82(2)$ & $74(2)$ & $75(2)$ & $17(2)$ & $9(2)$ & $-16(2)$ \\
\hline$C(19)$ & $93(3)$ & $88(3)$ & $85(3)$ & $11(2)$ & $10(2)$ & $-12(2)$ \\
\hline $\mathrm{C}(20)$ & $127(4)$ & 130(4) & $82(3)$ & $5(3)$ & $25(3)$ & $-22(3)$ \\
\hline $\mathrm{C}(21)$ & $115(4)$ & 183(6) & $102(4)$ & $40(4)$ & $30(3)$ & $-44(4)$ \\
\hline $\mathrm{C}(22)$ & $131(5)$ & $212(7)$ & 91(3) & $30(4)$ & $13(3)$ & $-91(5)$ \\
\hline$C(23)$ & $113(3)$ & $127(4)$ & $81(3)$ & $24(3)$ & $7(2)$ & $-55(3)$ \\
\hline $\mathrm{C}(24)$ & $120(4)$ & 111(4) & $106(3)$ & $-10(3)$ & $2(3)$ & $-27(3)$ \\
\hline$C(25)$ & $220(8)$ & 212(8) & $124(5)$ & $-9(5)$ & $-23(5)$ & $-87(7)$ \\
\hline$C(26)$ & $165(6)$ & $230(8)$ & $89(3)$ & $-7(4)$ & $4(3)$ & $-114(6)$ \\
\hline$C(27)$ & $244(10)$ & $114(5)$ & $190(7)$ & $-36(5)$ & $58(7)$ & $-28(6)$ \\
\hline
\end{tabular}




$\begin{array}{lcccccc}\mathrm{C}(28) & 72(2) & 50(2) & 53(2) & -5(1) & 14(1) & -3(1) \\ \mathrm{C}(29) & 74(2) & 50(2) & 57(2) & -5(1) & 18(1) & -4(1) \\ \mathrm{N}(4) & 87(2) & 51(2) & 55(1) & -7(1) & 21(1) & -9(1) \\ \mathrm{C}(30) & 85(2) & 64(2) & 58(2) & -14(2) & 23(2) & -11(2) \\ \mathrm{C}(31) & 91(2) & 42(2) & 62(2) & -6(1) & 20(2) & -3(2) \\ \mathrm{O}(3) & 118(2) & 47(1) & 68(1) & -12(1) & 33(1) & -17(1) \\ \mathrm{O}(4) & 139(2) & 79(2) & 68(1) & -22(1) & 41(2) & -32(2) \\ \mathrm{C}(32) & 94(3) & 60(2) & 51(2) & -1(2) & 21(2) & -7(2) \\ \mathrm{C}(33) & 105(3) & 58(2) & 61(2) & -1(2) & 20(2) & -3(2) \\ \mathrm{C}(34) & 131(4) & 65(2) & 70(2) & 6(2) & 20(2) & 2(2) \\ \mathrm{C}(35) & 140(4) & 73(3) & 75(2) & 15(2) & 25(3) & -23(3) \\ \mathrm{C}(36) & 119(3) & 95(3) & 70(2) & 7(2) & 26(2) & -34(3) \\ \mathrm{C}(37) & 92(3) & 78(2) & 62(2) & 2(2) & 18(2) & -18(2) \\ \mathrm{C}(38) & 97(3) & 88(3) & 80(2) & 3(2) & 14(2) & 5(2) \\ \mathrm{C}(39) & 135(5) & 174(6) & 140(5) & -39(5) & 16(4) & -37(5) \\ \mathrm{C}(40) & 91(3) & 115(4) & 83(3) & 12(3) & 13(2) & -16(3) \\ \mathrm{C}(41) & 101(3) & 133(4) & 117(4) & 1(3) & 27(3) & -6(3)\end{array}$

Table S5. Hydrogen coordinates ( x 104) and isotropic displacement parameters $\left(\AA^{2} \mathrm{x} 10^{3}\right)$ for AS440a_sq.

\begin{tabular}{|c|c|c|c|c|}
\hline & $\mathrm{x}$ & $\mathrm{y}$ & $\mathrm{z}$ & $\mathrm{U}(\mathrm{eq})$ \\
\hline $\mathrm{H}(3)$ & 8233 & 3097 & 6697 & 76 \\
\hline $\mathrm{H}(4)$ & 8652 & 3824 & 7154 & 92 \\
\hline $\mathrm{H}(5)$ & 7984 & 5290 & 7312 & 96 \\
\hline $\mathrm{H}(6)$ & 6978 & 6074 & 6991 & 83 \\
\hline $\mathrm{H}(10)$ & 4853 & 7896 & 6302 & 130 \\
\hline $\mathrm{H}(11)$ & 4028 & 7842 & 5854 & 144 \\
\hline $\mathrm{H}(12)$ & 4081 & 6495 & 5527 & 136 \\
\hline $\mathrm{H}(13)$ & 5022 & 5148 & 5638 & 105 \\
\hline $\mathrm{H}(16 \mathrm{~A})$ & 6620 & 2313 & 6111 & 72 \\
\hline $\mathrm{H}(16 \mathrm{~B})$ & 7287 & 2222 & 6394 & 72 \\
\hline $\mathrm{H}(3 \mathrm{~A})$ & 6324 & 1325 & 6679 & 91 \\
\hline $\mathrm{H}(20)$ & 4510 & 2338 & 7582 & 135 \\
\hline
\end{tabular}




\begin{tabular}{|c|c|c|c|c|}
\hline $\mathrm{H}(21)$ & 3320 & 1337 & 7445 & 160 \\
\hline $\mathrm{H}(22)$ & 3332 & 505 & 6987 & 174 \\
\hline $\mathrm{H}(24 \mathrm{~A})$ & 6593 & 2387 & 7221 & 135 \\
\hline $\mathrm{H}(24 \mathrm{~B})$ & 6042 & 3343 & 7140 & 135 \\
\hline $\mathrm{H}(25 \mathrm{~A})$ & 5597 & 3454 & 7655 & 278 \\
\hline $\mathrm{H}(25 \mathrm{~B})$ & 6652 & 3531 & 7615 & 278 \\
\hline $\mathrm{H}(25 \mathrm{C})$ & 6214 & 2536 & 7730 & 278 \\
\hline $\mathrm{H}(26 \mathrm{~A})$ & 4610 & 887 & 6360 & 193 \\
\hline $\mathrm{H}(26 \mathrm{~B})$ & 5153 & 60 & 6532 & 193 \\
\hline $\mathrm{H}(27 \mathrm{~A})$ & 3340 & 64 & 6418 & 274 \\
\hline $\mathrm{H}(27 \mathrm{~B})$ & 3824 & -733 & 6625 & 274 \\
\hline $\mathrm{H}(27 \mathrm{C})$ & 4074 & -640 & 6272 & 274 \\
\hline $\mathrm{H}(28)$ & 8473 & 3430 & 6167 & 70 \\
\hline $\mathrm{H}(31 \mathrm{~A})$ & 8330 & 2424 & 5780 & 78 \\
\hline $\mathrm{H}(31 \mathrm{~B})$ & 7327 & 2770 & 5712 & 78 \\
\hline $\mathrm{H}(34)$ & 7858 & 7186 & 4996 & 107 \\
\hline $\mathrm{H}(35)$ & 9362 & 7538 & 4959 & 115 \\
\hline $\mathrm{H}(36)$ & 10411 & 6561 & 5194 & 114 \\
\hline $\mathrm{H}(38 \mathrm{~A})$ & 6869 & 5411 & 5481 & 106 \\
\hline $\mathrm{H}(38 \mathrm{~B})$ & 6652 & 6348 & 5279 & 106 \\
\hline $\mathrm{H}(39 \mathrm{~A})$ & 6715 & 5430 & 4837 & 225 \\
\hline $\mathrm{H}(39 \mathrm{~B})$ & 6044 & 4913 & 5069 & 225 \\
\hline $\mathrm{H}(39 \mathrm{C})$ & 7028 & 4493 & 5025 & 225 \\
\hline $\mathrm{H}(40 \mathrm{~A})$ & 10112 & 5002 & 5779 & 116 \\
\hline $\mathrm{H}(40 \mathrm{~B})$ & 10075 & 4266 & 5499 & 116 \\
\hline $\mathrm{H}(41 \mathrm{~A})$ & 11297 & 5102 & 5276 & 175 \\
\hline $\mathrm{H}(41 \mathrm{~B})$ & 11540 & 4617 & 5597 & 175 \\
\hline $\mathrm{H}(41 \mathrm{C})$ & 11356 & 5762 & 5576 & 175 \\
\hline
\end{tabular}


Table S6. Torsion angles $\left[{ }^{\circ}\right]$ for AS440a_sq.

\begin{tabular}{|c|c|}
\hline$C(15)-C(1)-C(2)-C(7)$ & $-4.7(4)$ \\
\hline $\mathrm{C}(16)-\mathrm{C}(1)-\mathrm{C}(2)-\mathrm{C}(7)$ & $-126.3(3)$ \\
\hline $\mathrm{C}(28)-\mathrm{C}(1)-\mathrm{C}(2)-\mathrm{C}(7)$ & $115.0(3)$ \\
\hline$C(15)-C(1)-C(2)-C(3)$ & $174.9(3)$ \\
\hline$C(16)-C(1)-C(2)-C(3)$ & $53.3(4)$ \\
\hline $\mathrm{C}(28)-\mathrm{C}(1)-\mathrm{C}(2)-\mathrm{C}(3)$ & $-65.4(3)$ \\
\hline$C(7)-C(2)-C(3)-C(4)$ & $-0.8(5)$ \\
\hline$C(1)-C(2)-C(3)-C(4)$ & $179.6(3)$ \\
\hline$C(2)-C(3)-C(4)-C(5)$ & $2.2(6)$ \\
\hline$C(3)-C(4)-C(5)-C(6)$ & $-2.4(6)$ \\
\hline$C(4)-C(5)-C(6)-C(7)$ & $1.1(6)$ \\
\hline$C(5)-C(6)-C(7)-C(2)$ & $0.3(5)$ \\
\hline$C(5)-C(6)-C(7)-C(8)$ & $-179.2(3)$ \\
\hline$C(3)-C(2)-C(7)-C(6)$ & $-0.5(4)$ \\
\hline$C(1)-C(2)-C(7)-C(6)$ & 179.1(3) \\
\hline$C(3)-C(2)-C(7)-C(8)$ & 179.1(3) \\
\hline $\mathrm{C}(1)-\mathrm{C}(2)-\mathrm{C}(7)-\mathrm{C}(8)$ & $-1.3(4)$ \\
\hline $\mathrm{C}(6)-\mathrm{C}(7)-\mathrm{C}(8)-\mathrm{N}(1)$ & $3.9(5)$ \\
\hline $\mathrm{C}(2)-\mathrm{C}(7)-\mathrm{C}(8)-\mathrm{N}(1)$ & $-175.6(3)$ \\
\hline $\mathrm{C}(6)-\mathrm{C}(7)-\mathrm{C}(8)-\mathrm{N}(2)$ & $-176.0(3)$ \\
\hline $\mathrm{C}(2)-\mathrm{C}(7)-\mathrm{C}(8)-\mathrm{N}(2)$ & $4.5(4)$ \\
\hline $\mathrm{N}(2)-\mathrm{C}(8)-\mathrm{N}(1)-\mathrm{C}(9)$ & $0.7(4)$ \\
\hline $\mathrm{C}(7)-\mathrm{C}(8)-\mathrm{N}(1)-\mathrm{C}(9)$ & $-179.2(3)$ \\
\hline $\mathrm{C}(8)-\mathrm{N}(1)-\mathrm{C}(9)-\mathrm{C}(10)$ & $179.0(4)$ \\
\hline $\mathrm{C}(8)-\mathrm{N}(1)-\mathrm{C}(9)-\mathrm{C}(14)$ & $-0.3(4)$ \\
\hline $\mathrm{N}(1)-\mathrm{C}(9)-\mathrm{C}(10)-\mathrm{C}(11)$ & $-179.7(4)$ \\
\hline$C(14)-C(9)-C(10)-C(11)$ & $-0.3(7)$ \\
\hline$C(9)-C(10)-C(11)-C(12)$ & $0.9(8)$ \\
\hline $\mathrm{C}(10)-\mathrm{C}(11)-\mathrm{C}(12)-\mathrm{C}(13)$ & $-0.9(9)$ \\
\hline$C(11)-C(12)-C(13)-C(14)$ & $0.4(7)$ \\
\hline$C(12)-C(13)-C(14)-C(9)$ & $0.2(6)$ \\
\hline $\mathrm{C}(12)-\mathrm{C}(13)-\mathrm{C}(14)-\mathrm{N}(2)$ & $179.3(4)$ \\
\hline$C(10)-C(9)-C(14)-C(13)$ & $-0.2(6)$ \\
\hline $\mathrm{N}(1)-\mathrm{C}(9)-\mathrm{C}(14)-\mathrm{C}(13)$ & $179.2(3)$ \\
\hline $\mathrm{C}(10)-\mathrm{C}(9)-\mathrm{C}(14)-\mathrm{N}(2)$ & $-179.5(4)$ \\
\hline
\end{tabular}




\begin{tabular}{|c|c|}
\hline $\mathrm{N}(1)-\mathrm{C}(9)-\mathrm{C}(14)-\mathrm{N}(2)$ & $-0.1(4)$ \\
\hline $\mathrm{N}(1)-\mathrm{C}(8)-\mathrm{N}(2)-\mathrm{C}(15)$ & 179.1(3) \\
\hline $\mathrm{C}(7)-\mathrm{C}(8)-\mathrm{N}(2)-\mathrm{C}(15)$ & $-1.0(4)$ \\
\hline $\mathrm{N}(1)-\mathrm{C}(8)-\mathrm{N}(2)-\mathrm{C}(14)$ & $-0.8(4)$ \\
\hline $\mathrm{C}(7)-\mathrm{C}(8)-\mathrm{N}(2)-\mathrm{C}(14)$ & 179.1(3) \\
\hline$C(13)-C(14)-N(2)-C(15)$ & $1.4(6)$ \\
\hline $\mathrm{C}(9)-\mathrm{C}(14)-\mathrm{N}(2)-\mathrm{C}(15)$ & $-179.3(3)$ \\
\hline $\mathrm{C}(13)-\mathrm{C}(14)-\mathrm{N}(2)-\mathrm{C}(8)$ & $-178.8(4)$ \\
\hline $\mathrm{C}(9)-\mathrm{C}(14)-\mathrm{N}(2)-\mathrm{C}(8)$ & $0.5(3)$ \\
\hline $\mathrm{C}(8)-\mathrm{N}(2)-\mathrm{C}(15)-\mathrm{O}(1)$ & $179.0(3)$ \\
\hline $\mathrm{C}(14)-\mathrm{N}(2)-\mathrm{C}(15)-\mathrm{O}(1)$ & $-1.1(5)$ \\
\hline $\mathrm{C}(8)-\mathrm{N}(2)-\mathrm{C}(15)-\mathrm{C}(1)$ & $-5.3(4)$ \\
\hline $\mathrm{C}(14)-\mathrm{N}(2)-\mathrm{C}(15)-\mathrm{C}(1)$ & $174.5(3)$ \\
\hline $\mathrm{C}(2)-\mathrm{C}(1)-\mathrm{C}(15)-\mathrm{O}(1)$ & $-176.6(3)$ \\
\hline $\mathrm{C}(16)-\mathrm{C}(1)-\mathrm{C}(15)-\mathrm{O}(1)$ & $-53.4(4)$ \\
\hline $\mathrm{C}(28)-\mathrm{C}(1)-\mathrm{C}(15)-\mathrm{O}(1)$ & $62.4(4)$ \\
\hline $\mathrm{C}(2)-\mathrm{C}(1)-\mathrm{C}(15)-\mathrm{N}(2)$ & $7.8(4)$ \\
\hline $\mathrm{C}(16)-\mathrm{C}(1)-\mathrm{C}(15)-\mathrm{N}(2)$ & $131.0(3)$ \\
\hline $\mathrm{C}(28)-\mathrm{C}(1)-\mathrm{C}(15)-\mathrm{N}(2)$ & $-113.2(3)$ \\
\hline$C(15)-C(1)-C(16)-C(17)$ & $-68.2(3)$ \\
\hline$C(2)-C(1)-C(16)-C(17)$ & $57.9(4)$ \\
\hline$C(28)-C(1)-C(16)-C(17)$ & 177.1(3) \\
\hline $\mathrm{C}(1)-\mathrm{C}(16)-\mathrm{C}(17)-\mathrm{O}(2)$ & 29.4(4) \\
\hline $\mathrm{C}(1)-\mathrm{C}(16)-\mathrm{C}(17)-\mathrm{N}(3)$ & $-150.6(3)$ \\
\hline $\mathrm{O}(2)-\mathrm{C}(17)-\mathrm{N}(3)-\mathrm{C}(18)$ & $1.2(6)$ \\
\hline $\mathrm{C}(16)-\mathrm{C}(17)-\mathrm{N}(3)-\mathrm{C}(18)$ & $-178.8(3)$ \\
\hline $\mathrm{C}(17)-\mathrm{N}(3)-\mathrm{C}(18)-\mathrm{C}(23)$ & $104.8(5)$ \\
\hline $\mathrm{C}(17)-\mathrm{N}(3)-\mathrm{C}(18)-\mathrm{C}(19)$ & $-77.5(5)$ \\
\hline$C(23)-C(18)-C(19)-C(20)$ & $-4.6(7)$ \\
\hline $\mathrm{N}(3)-\mathrm{C}(18)-\mathrm{C}(19)-\mathrm{C}(20)$ & $177.9(4)$ \\
\hline$C(23)-C(18)-C(19)-C(24)$ & $175.1(5)$ \\
\hline $\mathrm{N}(3)-\mathrm{C}(18)-\mathrm{C}(19)-\mathrm{C}(24)$ & $-2.4(6)$ \\
\hline$C(18)-C(19)-C(20)-C(21)$ & $1.5(8)$ \\
\hline $\mathrm{C}(24)-\mathrm{C}(19)-\mathrm{C}(20)-\mathrm{C}(21)$ & $-178.2(5)$ \\
\hline$C(19)-C(20)-C(21)-C(22)$ & $0.9(10)$ \\
\hline $\mathrm{C}(20)-\mathrm{C}(21)-\mathrm{C}(22)-\mathrm{C}(23)$ & $-0.6(11)$ \\
\hline $\mathrm{C}(21)-\mathrm{C}(22)-\mathrm{C}(23)-\mathrm{C}(18)$ & $-2.2(10)$ \\
\hline
\end{tabular}




\begin{tabular}{|c|c|}
\hline$C(21)-C(22)-C(23)-C(26)$ & $176.0(7)$ \\
\hline$C(19)-C(18)-C(23)-C(22)$ & $4.9(8)$ \\
\hline $\mathrm{N}(3)-\mathrm{C}(18)-\mathrm{C}(23)-\mathrm{C}(22)$ & $-177.6(5)$ \\
\hline$C(19)-C(18)-C(23)-C(26)$ & $-173.4(5)$ \\
\hline $\mathrm{N}(3)-\mathrm{C}(18)-\mathrm{C}(23)-\mathrm{C}(26)$ & $4.2(7)$ \\
\hline$C(20)-C(19)-C(24)-C(25)$ & 11.3(9) \\
\hline $\mathrm{C}(18)-\mathrm{C}(19)-\mathrm{C}(24)-\mathrm{C}(25)$ & $-168.4(6)$ \\
\hline$C(22)-C(23)-C(26)-C(27)$ & $-1.2(12)$ \\
\hline $\mathrm{C}(18)-\mathrm{C}(23)-\mathrm{C}(26)-\mathrm{C}(27)$ & $176.9(7)$ \\
\hline $\mathrm{C}(15)-\mathrm{C}(1)-\mathrm{C}(28)-\mathrm{C}(31)$ & $-69.1(3)$ \\
\hline$C(2)-C(1)-C(28)-C(31)$ & $166.1(3)$ \\
\hline $\mathrm{C}(16)-\mathrm{C}(1)-\mathrm{C}(28)-\mathrm{C}(31)$ & $46.1(4)$ \\
\hline $\mathrm{C}(15)-\mathrm{C}(1)-\mathrm{C}(28)-\mathrm{C}(29)$ & $52.1(3)$ \\
\hline $\mathrm{C}(2)-\mathrm{C}(1)-\mathrm{C}(28)-\mathrm{C}(29)$ & $-72.7(3)$ \\
\hline $\mathrm{C}(16)-\mathrm{C}(1)-\mathrm{C}(28)-\mathrm{C}(29)$ & $167.3(3)$ \\
\hline $\mathrm{C}(31)-\mathrm{C}(28)-\mathrm{C}(29)-\mathrm{O}(3)$ & $175.7(4)$ \\
\hline $\mathrm{C}(1)-\mathrm{C}(28)-\mathrm{C}(29)-\mathrm{O}(3)$ & $46.4(5)$ \\
\hline $\mathrm{C}(31)-\mathrm{C}(28)-\mathrm{C}(29)-\mathrm{N}(4)$ & $-3.9(4)$ \\
\hline $\mathrm{C}(1)-\mathrm{C}(28)-\mathrm{C}(29)-\mathrm{N}(4)$ & $-133.1(3)$ \\
\hline $\mathrm{O}(3)-\mathrm{C}(29)-\mathrm{N}(4)-\mathrm{C}(30)$ & $179.1(4)$ \\
\hline $\mathrm{C}(28)-\mathrm{C}(29)-\mathrm{N}(4)-\mathrm{C}(30)$ & $-1.3(4)$ \\
\hline $\mathrm{O}(3)-\mathrm{C}(29)-\mathrm{N}(4)-\mathrm{C}(32)$ & $3.4(6)$ \\
\hline $\mathrm{C}(28)-\mathrm{C}(29)-\mathrm{N}(4)-\mathrm{C}(32)$ & $-177.1(3)$ \\
\hline $\mathrm{C}(29)-\mathrm{N}(4)-\mathrm{C}(30)-\mathrm{O}(4)$ & $-175.7(4)$ \\
\hline $\mathrm{C}(32)-\mathrm{N}(4)-\mathrm{C}(30)-\mathrm{O}(4)$ & $0.0(6)$ \\
\hline $\mathrm{C}(29)-\mathrm{N}(4)-\mathrm{C}(30)-\mathrm{C}(31)$ & $6.2(4)$ \\
\hline $\mathrm{C}(32)-\mathrm{N}(4)-\mathrm{C}(30)-\mathrm{C}(31)$ & $-178.1(3)$ \\
\hline $\mathrm{O}(4)-\mathrm{C}(30)-\mathrm{C}(31)-\mathrm{C}(28)$ & $173.8(4)$ \\
\hline $\mathrm{N}(4)-\mathrm{C}(30)-\mathrm{C}(31)-\mathrm{C}(28)$ & $-8.3(4)$ \\
\hline $\mathrm{C}(29)-\mathrm{C}(28)-\mathrm{C}(31)-\mathrm{C}(30)$ & $7.2(4)$ \\
\hline $\mathrm{C}(1)-\mathrm{C}(28)-\mathrm{C}(31)-\mathrm{C}(30)$ & $132.0(3)$ \\
\hline $\mathrm{C}(29)-\mathrm{N}(4)-\mathrm{C}(32)-\mathrm{C}(33)$ & $-92.3(4)$ \\
\hline $\mathrm{C}(30)-\mathrm{N}(4)-\mathrm{C}(32)-\mathrm{C}(33)$ & $92.4(4)$ \\
\hline $\mathrm{C}(29)-\mathrm{N}(4)-\mathrm{C}(32)-\mathrm{C}(37)$ & $86.5(4)$ \\
\hline $\mathrm{C}(30)-\mathrm{N}(4)-\mathrm{C}(32)-\mathrm{C}(37)$ & $-88.8(4)$ \\
\hline $\mathrm{C}(37)-\mathrm{C}(32)-\mathrm{C}(33)-\mathrm{C}(34)$ & $-1.4(5)$ \\
\hline $\mathrm{N}(4)-\mathrm{C}(32)-\mathrm{C}(33)-\mathrm{C}(34)$ & $177.3(3)$ \\
\hline
\end{tabular}




$\begin{array}{lc}\mathrm{C}(37)-\mathrm{C}(32)-\mathrm{C}(33)-\mathrm{C}(38) & 178.4(4) \\ \mathrm{N}(4)-\mathrm{C}(32)-\mathrm{C}(33)-\mathrm{C}(38) & -2.9(5) \\ \mathrm{C}(32)-\mathrm{C}(33)-\mathrm{C}(34)-\mathrm{C}(35) & 1.2(6) \\ \mathrm{C}(38)-\mathrm{C}(33)-\mathrm{C}(34)-\mathrm{C}(35) & -178.5(4) \\ \mathrm{C}(33)-\mathrm{C}(34)-\mathrm{C}(35)-\mathrm{C}(36) & 0.2(7) \\ \mathrm{C}(34)-\mathrm{C}(35)-\mathrm{C}(36)-\mathrm{C}(37) & -1.6(7) \\ \mathrm{C}(35)-\mathrm{C}(36)-\mathrm{C}(37)-\mathrm{C}(32) & 1.5(6) \\ \mathrm{C}(35)-\mathrm{C}(36)-\mathrm{C}(37)-\mathrm{C}(40) & 179.5(4) \\ \mathrm{C}(33)-\mathrm{C}(32)-\mathrm{C}(37)-\mathrm{C}(36) & 0.0(5) \\ \mathrm{N}(4)-\mathrm{C}(32)-\mathrm{C}(37)-\mathrm{C}(36) & -178.7(3) \\ \mathrm{C}(33)-\mathrm{C}(32)-\mathrm{C}(37)-\mathrm{C}(40) & -178.0(4) \\ \mathrm{N}(4)-\mathrm{C}(32)-\mathrm{C}(37)-\mathrm{C}(40) & 3.2(5) \\ \mathrm{C}(32)-\mathrm{C}(33)-\mathrm{C}(38)-\mathrm{C}(39) & -91.8(6) \\ \mathrm{C}(34)-\mathrm{C}(33)-\mathrm{C}(38)-\mathrm{C}(39) & 87.9(6) \\ \mathrm{C}(36)-\mathrm{C}(37)-\mathrm{C}(40)-\mathrm{C}(41) & -5.2(7) \\ \mathrm{C}(32)-\mathrm{C}(37)-\mathrm{C}(40)-\mathrm{C}(41) & 172.7(4)\end{array}$

Symmetry transformations used to generate equivalent atoms: 


\section{Crystal refinement data for compound $4 \mathrm{e}$}

Empirical Formula- $\mathrm{C}_{19} \mathrm{H}_{15} \mathrm{~N}_{3} \mathrm{O}_{2}, \mathrm{M}=317.34$, Monoclinic, Space group $\mathrm{P} 21 / \mathrm{c}, \mathrm{a}=$ 14.129(18) $\AA, \mathrm{b}=10.513(10) \AA, \mathrm{c}=10.840(10) \AA, \mathrm{V}=1510(3) \AA^{3}, \mathrm{Z}=4, \mathrm{~T}=223(2)$ $\mathrm{K}$, pcalcd $=1.396 \mathrm{Mg} / \mathrm{m}^{3}, 2 \Theta_{\max .}=28.343^{\circ}$, Refinement of 664 parameters on 3763 independent reflections out of 18435 collected reflections $\left(R_{\text {int }}=0.0819\right)$ led to $R_{1}=$ $0.0555[\mathrm{I}>2 \sigma(\mathrm{I})], \mathrm{wR}_{2}=0.1833$ (all data) and $\mathrm{S}=1.004$ with the largest difference peak and hole of 0.178 and $-0.222 \mathrm{e} . \AA^{-3}$ respectively. The crystal structure has been deposited at the Cambridge Crystallographic Data Centre (CCDC 2085989). The data can be obtained free of charge via the Internet at www.ccdc.cam.ac.uk/data_request/cif

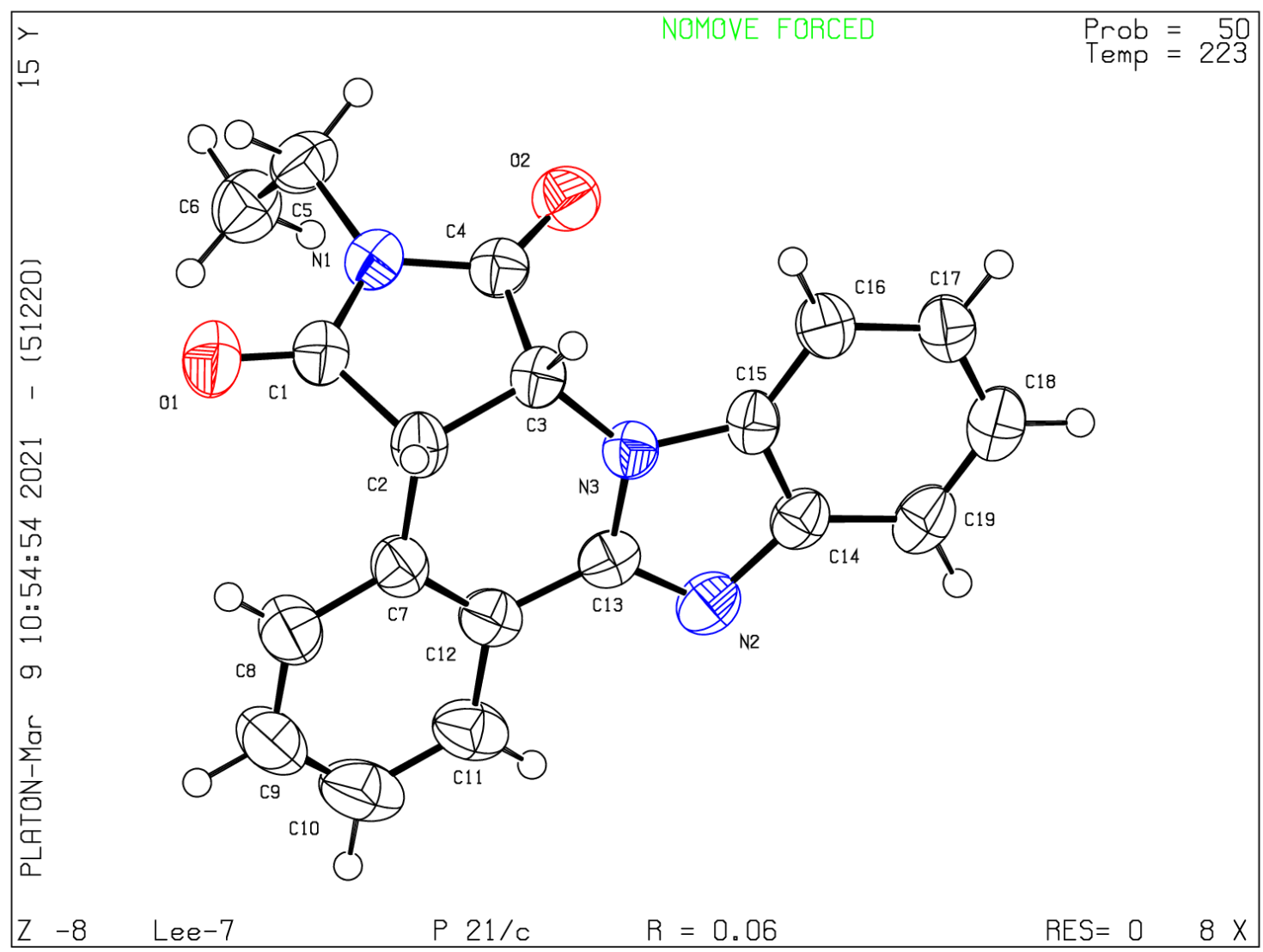

Fig. S8. X-ray structure of compound $\mathbf{4 e}$ (ellipsoid contour \% probability-50). 
Table S7. Crystal data and structure refinement for (4e) Lee-7.

Identification code

Empirical formula

Formula weight

Temperature

Wavelength

Crystal system

Space group

Unit cell dimensions

Volume

$\mathrm{Z}$

Density (calculated)

Absorption coefficient

$\mathrm{F}(000)$

Crystal size

Theta range for data collection

Index ranges

Reflections collected

Independent reflections

Completeness to theta $=25.242^{\circ}$

Absorption correction

Max. and min. transmission

Refinement method

Data / restraints / parameters

Goodness-of-fit on $\mathrm{F}^{2}$

Final $\mathrm{R}$ indices [I $>2 \operatorname{sigma}(\mathrm{I})]$

$\mathrm{R}$ indices (all data)

Extinction coefficient

Largest diff. peak and hole
Lee-7

C19 H15 N3 O2

317.34

223(2) K

$0.71073 \AA$

Monoclinic

$\mathrm{P} 21 / \mathrm{c}$

$\mathrm{a}=14.129(18) \AA \quad \alpha=90^{\circ}$.

$\mathrm{b}=10.513(10) \AA \quad \beta=110.36(4)^{\circ}$.

$\mathrm{c}=10.840(10) \AA \quad \gamma=90^{\circ}$.

1510(3) $\AA^{3}$

4

$1.396 \mathrm{Mg} / \mathrm{m}^{3}$

$0.093 \mathrm{~mm}^{-1}$

664

$0.399 \times 0.090 \times 0.053 \mathrm{~mm}^{3}$

2.473 to $28.343^{\circ}$.

$-18<=\mathrm{h}<=18,-14<=\mathrm{k}<=14,-10<=\mathrm{l}<=14$

18435

$3763[\mathrm{R}(\mathrm{int})=0.0819]$

$99.9 \%$

Semi-empirical from equivalents

0.7457 and 0.6080

Full-matrix least-squares on $\mathrm{F}^{2}$

3763 / 0 / 218

1.004

$\mathrm{R} 1=0.0555, \mathrm{wR} 2=0.1366$

$\mathrm{R} 1=0.1343, \mathrm{wR} 2=0.1833$

$\mathrm{n} / \mathrm{a}$

0.178 and -0.222 e. $\AA^{-3}$ 
Table S8. Atomic coordinates $\left(\mathrm{x} 10^{4}\right)$ and equivalent isotropic displacement parameters $\left(\AA^{2} \mathrm{x}\right.$ $10^{3}$ )

for Lee-7. $\quad \mathrm{U}(\mathrm{eq})$ is defined as one third of the trace of the orthogonalized $\mathrm{U}^{\mathrm{ij}}$ tensor.

\begin{tabular}{|c|c|c|c|c|}
\hline & $\mathrm{x}$ & $\mathrm{y}$ & $\mathrm{z}$ & $\mathrm{U}(\mathrm{eq})$ \\
\hline $\mathrm{N}(1)$ & $2277(1)$ & $8539(2)$ & $5308(2)$ & $44(1)$ \\
\hline $\mathrm{C}(1)$ & $2116(2)$ & $7555(2)$ & $6050(2)$ & $47(1)$ \\
\hline$C(2)$ & $2813(2)$ & $6460(2)$ & $6034(2)$ & $45(1)$ \\
\hline $\mathrm{C}(3)$ & $3550(2)$ & $7057(2)$ & $5459(2)$ & $40(1)$ \\
\hline $\mathrm{C}(4)$ & $3068(2)$ & $8322(2)$ & $4885(2)$ & $42(1)$ \\
\hline$C(5)$ & $1669(2)$ & $9691(2)$ & $5018(3)$ & $54(1)$ \\
\hline$C(6)$ & $670(2)$ & $9465(2)$ & $3926(3)$ & $61(1)$ \\
\hline $\mathrm{O}(1)$ & $1496(1)$ & $7586(2)$ & $6591(2)$ & $68(1)$ \\
\hline $\mathrm{O}(2)$ & $3339(1)$ & $9029(2)$ & 4194(2) & $54(1)$ \\
\hline $\mathrm{C}(7)$ & $2205(2)$ & $5330(2)$ & $5287(2)$ & $45(1)$ \\
\hline $\mathrm{C}(8)$ & $1476(2)$ & $4780(3)$ & $5707(3)$ & $65(1)$ \\
\hline $\mathrm{C}(9)$ & $932(2)$ & $3746(3)$ & $5063(4)$ & $72(1)$ \\
\hline$C(10)$ & $1107(2)$ & $3237(3)$ & 3996(3) & $70(1)$ \\
\hline$C(11)$ & $1830(2)$ & $3753(2)$ & $3577(3)$ & $56(1)$ \\
\hline $\mathrm{C}(12)$ & $2384(2)$ & $4803(2)$ & $4214(2)$ & $44(1)$ \\
\hline$C(13)$ & $3202(2)$ & $5280(2)$ & $3835(2)$ & $41(1)$ \\
\hline $\mathrm{N}(2)$ & $3542(1)$ & $4789(2)$ & $2952(2)$ & $48(1)$ \\
\hline $\mathrm{N}(3)$ & $3772(1)$ & $6290(2)$ & $4495(2)$ & $39(1)$ \\
\hline$C(14)$ & $4384(2)$ & $5514(2)$ & $3041(2)$ & $43(1)$ \\
\hline$C(15)$ & $4539(2)$ & $6459(2)$ & $3998(2)$ & $40(1)$ \\
\hline$C(16)$ & $5339(2)$ & $7309(2)$ & $4299(2)$ & $47(1)$ \\
\hline $\mathrm{C}(17)$ & 5982(2) & $7188(2)$ & $3609(2)$ & $49(1)$ \\
\hline$C(18)$ & $5845(2)$ & $6255(2)$ & $2651(2)$ & $54(1)$ \\
\hline $\mathrm{C}(19)$ & $5050(2)$ & $5413(2)$ & $2355(2)$ & $51(1)$ \\
\hline
\end{tabular}


Table S9. Bond lengths $[\AA]$ and angles $\left[{ }^{\circ}\right]$ for Lee-7.

\begin{tabular}{|c|c|}
\hline $\mathrm{N}(1)-\mathrm{C}(4)$ & $1.366(3)$ \\
\hline $\mathrm{N}(1)-\mathrm{C}(1)$ & $1.377(3)$ \\
\hline $\mathrm{N}(1)-\mathrm{C}(5)$ & $1.455(3)$ \\
\hline $\mathrm{C}(1)-\mathrm{O}(1)$ & $1.211(3)$ \\
\hline $\mathrm{C}(1)-\mathrm{C}(2)$ & $1.519(3)$ \\
\hline $\mathrm{C}(2)-\mathrm{C}(3)$ & $1.523(3)$ \\
\hline$C(2)-C(7)$ & $1.524(3)$ \\
\hline $\mathrm{C}(2)-\mathrm{H}(2)$ & 0.9900 \\
\hline $\mathrm{C}(3)-\mathrm{N}(3)$ & $1.439(3)$ \\
\hline$C(3)-C(4)$ & $1.525(3)$ \\
\hline $\mathrm{C}(3)-\mathrm{H}(3)$ & 0.9900 \\
\hline $\mathrm{C}(4)-\mathrm{O}(2)$ & $1.209(3)$ \\
\hline$C(5)-C(6)$ & $1.513(3)$ \\
\hline $\mathrm{C}(5)-\mathrm{H}(5 \mathrm{~A})$ & 0.9800 \\
\hline $\mathrm{C}(5)-\mathrm{H}(5 \mathrm{~B})$ & 0.9800 \\
\hline $\mathrm{C}(6)-\mathrm{H}(6 \mathrm{~A})$ & 0.9700 \\
\hline $\mathrm{C}(6)-\mathrm{H}(6 \mathrm{~B})$ & 0.9700 \\
\hline $\mathrm{C}(6)-\mathrm{H}(6 \mathrm{C})$ & 0.9700 \\
\hline$C(7)-C(12)$ & $1.388(4)$ \\
\hline$C(7)-C(8)$ & $1.390(4)$ \\
\hline $\mathrm{C}(8)-\mathrm{C}(9)$ & $1.374(4)$ \\
\hline $\mathrm{C}(8)-\mathrm{H}(8)$ & 0.9400 \\
\hline $\mathrm{C}(9)-\mathrm{C}(10)$ & $1.373(4)$ \\
\hline $\mathrm{C}(9)-\mathrm{H}(9)$ & 0.9400 \\
\hline $\mathrm{C}(10)-\mathrm{C}(11)$ & $1.367(4)$ \\
\hline $\mathrm{C}(10)-\mathrm{H}(10)$ & 0.9400 \\
\hline $\mathrm{C}(11)-\mathrm{C}(12)$ & $1.390(3)$ \\
\hline $\mathrm{C}(11)-\mathrm{H}(11)$ & 0.9400 \\
\hline$C(12)-C(13)$ & $1.445(4)$ \\
\hline $\mathrm{C}(13)-\mathrm{N}(2)$ & $1.316(3)$ \\
\hline $\mathrm{C}(13)-\mathrm{N}(3)$ & $1.373(3)$ \\
\hline $\mathrm{N}(2)-\mathrm{C}(14)$ & $1.388(3)$ \\
\hline $\mathrm{N}(3)-\mathrm{C}(15)$ & $1.380(3)$ \\
\hline$C(14)-C(19)$ & $1.392(3)$ \\
\hline$C(14)-C(15)$ & $1.398(3)$ \\
\hline
\end{tabular}




\begin{tabular}{|c|c|}
\hline$C(15)-C(16)$ & $1.387(3)$ \\
\hline$C(16)-C(17)$ & $1.369(3)$ \\
\hline $\mathrm{C}(16)-\mathrm{H}(16)$ & 0.9400 \\
\hline $\mathrm{C}(17)-\mathrm{C}(18)$ & $1.392(3)$ \\
\hline $\mathrm{C}(17)-\mathrm{H}(17)$ & 0.9400 \\
\hline $\mathrm{C}(18)-\mathrm{C}(19)$ & $1.377(4)$ \\
\hline $\mathrm{C}(18)-\mathrm{H}(18)$ & 0.9400 \\
\hline C(19)-H(19) & 0.9400 \\
\hline $\mathrm{C}(4)-\mathrm{N}(1)-\mathrm{C}(1)$ & $113.0(2)$ \\
\hline $\mathrm{C}(4)-\mathrm{N}(1)-\mathrm{C}(5)$ & $123.9(2)$ \\
\hline $\mathrm{C}(1)-\mathrm{N}(1)-\mathrm{C}(5)$ & $123.1(2)$ \\
\hline $\mathrm{O}(1)-\mathrm{C}(1)-\mathrm{N}(1)$ & $123.8(2)$ \\
\hline $\mathrm{O}(1)-\mathrm{C}(1)-\mathrm{C}(2)$ & $127.3(2)$ \\
\hline $\mathrm{N}(1)-\mathrm{C}(1)-\mathrm{C}(2)$ & $108.8(2)$ \\
\hline $\mathrm{C}(1)-\mathrm{C}(2)-\mathrm{C}(3)$ & $103.3(2)$ \\
\hline $\mathrm{C}(1)-\mathrm{C}(2)-\mathrm{C}(7)$ & $110.4(2)$ \\
\hline$C(3)-C(2)-C(7)$ & $116.3(2)$ \\
\hline $\mathrm{C}(1)-\mathrm{C}(2)-\mathrm{H}(2)$ & 108.8 \\
\hline $\mathrm{C}(3)-\mathrm{C}(2)-\mathrm{H}(2)$ & 108.8 \\
\hline $\mathrm{C}(7)-\mathrm{C}(2)-\mathrm{H}(2)$ & 108.8 \\
\hline $\mathrm{N}(3)-\mathrm{C}(3)-\mathrm{C}(2)$ & $114.78(19)$ \\
\hline $\mathrm{N}(3)-\mathrm{C}(3)-\mathrm{C}(4)$ & $111.84(18)$ \\
\hline$C(2)-C(3)-C(4)$ & 104.71(19) \\
\hline $\mathrm{N}(3)-\mathrm{C}(3)-\mathrm{H}(3)$ & 108.4 \\
\hline $\mathrm{C}(2)-\mathrm{C}(3)-\mathrm{H}(3)$ & 108.4 \\
\hline $\mathrm{C}(4)-\mathrm{C}(3)-\mathrm{H}(3)$ & 108.4 \\
\hline $\mathrm{O}(2)-\mathrm{C}(4)-\mathrm{N}(1)$ & $125.3(2)$ \\
\hline $\mathrm{O}(2)-\mathrm{C}(4)-\mathrm{C}(3)$ & $126.6(2)$ \\
\hline $\mathrm{N}(1)-\mathrm{C}(4)-\mathrm{C}(3)$ & $108.1(2)$ \\
\hline $\mathrm{N}(1)-\mathrm{C}(5)-\mathrm{C}(6)$ & $111.0(2)$ \\
\hline $\mathrm{N}(1)-\mathrm{C}(5)-\mathrm{H}(5 \mathrm{~A})$ & 109.4 \\
\hline $\mathrm{C}(6)-\mathrm{C}(5)-\mathrm{H}(5 \mathrm{~A})$ & 109.4 \\
\hline $\mathrm{N}(1)-\mathrm{C}(5)-\mathrm{H}(5 \mathrm{~B})$ & 109.4 \\
\hline $\mathrm{C}(6)-\mathrm{C}(5)-\mathrm{H}(5 \mathrm{~B})$ & 109.4 \\
\hline $\mathrm{H}(5 \mathrm{~A})-\mathrm{C}(5)-\mathrm{H}(5 \mathrm{~B})$ & 108.0 \\
\hline $\mathrm{C}(5)-\mathrm{C}(6)-\mathrm{H}(6 \mathrm{~A})$ & 109.5 \\
\hline $\mathrm{C}(5)-\mathrm{C}(6)-\mathrm{H}(6 \mathrm{~B})$ & 109.5 \\
\hline
\end{tabular}




\begin{tabular}{|c|c|}
\hline $\mathrm{H}(6 \mathrm{~A})-\mathrm{C}(6)-\mathrm{H}(6 \mathrm{~B})$ & 109.5 \\
\hline $\mathrm{C}(5)-\mathrm{C}(6)-\mathrm{H}(6 \mathrm{C})$ & 109.5 \\
\hline $\mathrm{H}(6 \mathrm{~A})-\mathrm{C}(6)-\mathrm{H}(6 \mathrm{C})$ & 109.5 \\
\hline $\mathrm{H}(6 \mathrm{~B})-\mathrm{C}(6)-\mathrm{H}(6 \mathrm{C})$ & 109.5 \\
\hline $\mathrm{C}(12)-\mathrm{C}(7)-\mathrm{C}(8)$ & $118.7(2)$ \\
\hline$C(12)-C(7)-C(2)$ & $121.6(2)$ \\
\hline $\mathrm{C}(8)-\mathrm{C}(7)-\mathrm{C}(2)$ & 119.7(2) \\
\hline $\mathrm{C}(9)-\mathrm{C}(8)-\mathrm{C}(7)$ & $120.7(3)$ \\
\hline $\mathrm{C}(9)-\mathrm{C}(8)-\mathrm{H}(8)$ & 119.7 \\
\hline $\mathrm{C}(7)-\mathrm{C}(8)-\mathrm{H}(8)$ & 119.7 \\
\hline $\mathrm{C}(10)-\mathrm{C}(9)-\mathrm{C}(8)$ & $120.3(3)$ \\
\hline $\mathrm{C}(10)-\mathrm{C}(9)-\mathrm{H}(9)$ & 119.9 \\
\hline $\mathrm{C}(8)-\mathrm{C}(9)-\mathrm{H}(9)$ & 119.9 \\
\hline $\mathrm{C}(11)-\mathrm{C}(10)-\mathrm{C}(9)$ & $120.0(3)$ \\
\hline $\mathrm{C}(11)-\mathrm{C}(10)-\mathrm{H}(10)$ & 120.0 \\
\hline $\mathrm{C}(9)-\mathrm{C}(10)-\mathrm{H}(10)$ & 120.0 \\
\hline $\mathrm{C}(10)-\mathrm{C}(11)-\mathrm{C}(12)$ & $120.5(3)$ \\
\hline $\mathrm{C}(10)-\mathrm{C}(11)-\mathrm{H}(11)$ & 119.8 \\
\hline $\mathrm{C}(12)-\mathrm{C}(11)-\mathrm{H}(11)$ & 119.8 \\
\hline$C(7)-C(12)-C(11)$ & $119.9(2)$ \\
\hline$C(7)-C(12)-C(13)$ & $119.8(2)$ \\
\hline $\mathrm{C}(11)-\mathrm{C}(12)-\mathrm{C}(13)$ & $120.1(2)$ \\
\hline $\mathrm{N}(2)-\mathrm{C}(13)-\mathrm{N}(3)$ & $112.7(2)$ \\
\hline $\mathrm{N}(2)-\mathrm{C}(13)-\mathrm{C}(12)$ & $126.9(2)$ \\
\hline $\mathrm{N}(3)-\mathrm{C}(13)-\mathrm{C}(12)$ & $120.1(2)$ \\
\hline $\mathrm{C}(13)-\mathrm{N}(2)-\mathrm{C}(14)$ & $104.7(2)$ \\
\hline $\mathrm{C}(13)-\mathrm{N}(3)-\mathrm{C}(15)$ & $107.08(19)$ \\
\hline $\mathrm{C}(13)-\mathrm{N}(3)-\mathrm{C}(3)$ & $125.2(2)$ \\
\hline $\mathrm{C}(15)-\mathrm{N}(3)-\mathrm{C}(3)$ & $127.64(18)$ \\
\hline $\mathrm{N}(2)-\mathrm{C}(14)-\mathrm{C}(19)$ & $130.1(2)$ \\
\hline $\mathrm{N}(2)-\mathrm{C}(14)-\mathrm{C}(15)$ & $110.6(2)$ \\
\hline$C(19)-C(14)-C(15)$ & $119.3(2)$ \\
\hline $\mathrm{N}(3)-\mathrm{C}(15)-\mathrm{C}(16)$ & $132.6(2)$ \\
\hline $\mathrm{N}(3)-\mathrm{C}(15)-\mathrm{C}(14)$ & $104.9(2)$ \\
\hline$C(16)-C(15)-C(14)$ & $122.5(2)$ \\
\hline$C(17)-C(16)-C(15)$ & $116.9(2)$ \\
\hline $\mathrm{C}(17)-\mathrm{C}(16)-\mathrm{H}(16)$ & 121.5 \\
\hline
\end{tabular}




$\begin{array}{ll}\mathrm{C}(15)-\mathrm{C}(16)-\mathrm{H}(16) & 121.5 \\ \mathrm{C}(16)-\mathrm{C}(17)-\mathrm{C}(18) & 121.7(2) \\ \mathrm{C}(16)-\mathrm{C}(17)-\mathrm{H}(17) & 119.1 \\ \mathrm{C}(18)-\mathrm{C}(17)-\mathrm{H}(17) & 119.1 \\ \mathrm{C}(19)-\mathrm{C}(18)-\mathrm{C}(17) & 121.2(3) \\ \mathrm{C}(19)-\mathrm{C}(18)-\mathrm{H}(18) & 119.4 \\ \mathrm{C}(17)-\mathrm{C}(18)-\mathrm{H}(18) & 119.4 \\ \mathrm{C}(18)-\mathrm{C}(19)-\mathrm{C}(14) & 118.3(2) \\ \mathrm{C}(18)-\mathrm{C}(19)-\mathrm{H}(19) & 120.8 \\ \mathrm{C}(14)-\mathrm{C}(19)-\mathrm{H}(19) & 120.8\end{array}$

Symmetry transformations used to generate equivalent atoms:

Table S10. Anisotropic displacement parameters $\left(\AA^{2} \mathrm{x} 10^{3}\right)$ for Lee-7. The anisotropic displacement factor exponent takes the form: $\quad-2 \pi^{2}\left[\mathrm{~h}^{2} \mathrm{a}^{* 2} \mathrm{U}^{11}+\ldots+2 \mathrm{~h} \mathrm{k} \mathrm{a}^{*} \mathrm{~b}^{*} \mathrm{U}^{12}\right]$

\begin{tabular}{|c|c|c|c|c|c|c|}
\hline & $\mathrm{U}^{11}$ & $\mathrm{U}^{22}$ & $\mathrm{U}^{33}$ & $\mathrm{U}^{23}$ & $\mathrm{U}^{13}$ & $\mathrm{U}^{12}$ \\
\hline $\mathrm{N}(1)$ & $43(1)$ & $45(1)$ & $45(1)$ & $-1(1)$ & $15(1)$ & $4(1)$ \\
\hline $\mathrm{C}(1)$ & $45(1)$ & $52(1)$ & $48(1)$ & $-1(1)$ & $20(1)$ & $1(1)$ \\
\hline $\mathrm{C}(2)$ & $43(1)$ & $53(1)$ & $41(1)$ & $5(1)$ & $17(1)$ & $3(1)$ \\
\hline$C(3)$ & $38(1)$ & $47(1)$ & $34(1)$ & $-1(1)$ & 11(1) & $2(1)$ \\
\hline $\mathrm{C}(4)$ & $43(1)$ & $45(1)$ & $35(1)$ & $-5(1)$ & 11(1) & $-2(1)$ \\
\hline$C(5)$ & $51(2)$ & $46(1)$ & $61(2)$ & $-5(1)$ & $15(1)$ & $8(1)$ \\
\hline$C(6)$ & $51(2)$ & $65(2)$ & $61(2)$ & $0(1)$ & $13(1)$ & $7(1)$ \\
\hline $\mathrm{O}(1)$ & $68(1)$ & $71(1)$ & $84(1)$ & $3(1)$ & $48(1)$ & $7(1)$ \\
\hline $\mathrm{O}(2)$ & $67(1)$ & $50(1)$ & $50(1)$ & $5(1)$ & $26(1)$ & 1(1) \\
\hline$C(7)$ & $39(1)$ & $46(1)$ & $51(1)$ & $10(1)$ & $16(1)$ & $4(1)$ \\
\hline$C(8)$ & $55(2)$ & $59(2)$ & $86(2)$ & $11(2)$ & $33(2)$ & $2(1)$ \\
\hline $\mathrm{C}(9)$ & $54(2)$ & $59(2)$ & $104(3)$ & $18(2)$ & $30(2)$ & $-7(1)$ \\
\hline$C(10)$ & $53(2)$ & $50(2)$ & $92(2)$ & $9(2)$ & $4(2)$ & $-9(1)$ \\
\hline $\mathrm{C}(11)$ & $50(2)$ & $48(1)$ & $60(2)$ & $4(1)$ & $5(1)$ & $-6(1)$ \\
\hline $\mathrm{C}(12)$ & $38(1)$ & $41(1)$ & $46(1)$ & $6(1)$ & $6(1)$ & $2(1)$ \\
\hline $\mathrm{C}(13)$ & $41(1)$ & $40(1)$ & $36(1)$ & $2(1)$ & $6(1)$ & $5(1)$ \\
\hline $\mathrm{N}(2)$ & $52(1)$ & $48(1)$ & $42(1)$ & $-2(1)$ & $14(1)$ & $6(1)$ \\
\hline $\mathrm{N}(3)$ & $39(1)$ & $42(1)$ & $37(1)$ & $-2(1)$ & $13(1)$ & $0(1)$ \\
\hline $\mathrm{C}(14)$ & $45(1)$ & $43(1)$ & $40(1)$ & $4(1)$ & $14(1)$ & $9(1)$ \\
\hline
\end{tabular}




$\begin{array}{lllllll}\mathrm{C}(15) & 38(1) & 49(1) & 35(1) & 7(1) & 14(1) & 7(1) \\ \mathrm{C}(16) & 43(1) & 53(1) & 44(1) & 2(1) & 15(1) & 0(1) \\ \mathrm{C}(17) & 43(1) & 55(1) & 51(1) & 10(1) & 19(1) & 5(1) \\ \mathrm{C}(18) & 54(2) & 63(2) & 49(1) & 15(1) & 25(1) & 18(1) \\ \mathrm{C}(19) & 59(2) & 54(2) & 42(1) & 5(1) & 20(1) & 17(1)\end{array}$

Table S11. Hydrogen coordinates ( x 104) and isotropic displacement parameters $\left(\AA^{2} \times 10^{3}\right)$ for Lee-7.

\begin{tabular}{lrrrl}
\hline & $\mathrm{x}$ & $\mathrm{y}$ & $\mathrm{z}$ & $\mathrm{U}(\mathrm{eq})$ \\
\hline $\mathrm{H}(2)$ & & & & \\
$\mathrm{H}(3)$ & 3188 & 6198 & 6952 & 54 \\
$\mathrm{H}(5 \mathrm{~A})$ & 4190 & 7235 & 6188 & 48 \\
$\mathrm{H}(5 B)$ & 10367 & 4756 & 65 \\
$\mathrm{H}(6 \mathrm{~A})$ & 2038 & 9972 & 5811 & 65 \\
$\mathrm{H}(6 \mathrm{~B})$ & 1546 & 3143 & 92 \\
$\mathrm{H}(6 \mathrm{C})$ & 791 & 3734 & 92 \\
$\mathrm{H}(8)$ & 287 & 10251 & 4199 & 92 \\
$\mathrm{H}(9)$ & 292 & 8822 & 6440 & 78 \\
$\mathrm{H}(10)$ & 1354 & 5119 & 5354 & 86 \\
$\mathrm{H}(11)$ & 438 & 3386 & 3554 & 85 \\
$\mathrm{H}(16)$ & 729 & 2533 & 2853 & 68 \\
$\mathrm{H}(17)$ & 1954 & 3395 & 4947 & 56 \\
$\mathrm{H}(18)$ & 5435 & 7938 & 3786 & 59 \\
$\mathrm{H}(19)$ & 6530 & 7751 & 2199 & 64 \\
& 6303 & 6198 & 1706 & 61 \\
\hline
\end{tabular}

Table S12. Torsion angles $\left[{ }^{\circ}\right]$ for Lee-7.

\begin{tabular}{lc}
\hline $\mathrm{C}(4)-\mathrm{N}(1)-\mathrm{C}(1)-\mathrm{O}(1)$ & $175.2(2)$ \\
$\mathrm{C}(5)-\mathrm{N}(1)-\mathrm{C}(1)-\mathrm{O}(1)$ & $-4.4(4)$ \\
$\mathrm{C}(4)-\mathrm{N}(1)-\mathrm{C}(1)-\mathrm{C}(2)$ & $-6.2(2)$ \\
$\mathrm{C}(5)-\mathrm{N}(1)-\mathrm{C}(1)-\mathrm{C}(2)$ & $174.21(18)$ \\
$\mathrm{O}(1)-\mathrm{C}(1)-\mathrm{C}(2)-\mathrm{C}(3)$ & $-168.9(2)$
\end{tabular}




\begin{tabular}{|c|c|}
\hline $\mathrm{N}(1)-\mathrm{C}(1)-\mathrm{C}(2)-\mathrm{C}(3)$ & $12.5(2)$ \\
\hline $\mathrm{O}(1)-\mathrm{C}(1)-\mathrm{C}(2)-\mathrm{C}(7)$ & $66.1(3)$ \\
\hline $\mathrm{N}(1)-\mathrm{C}(1)-\mathrm{C}(2)-\mathrm{C}(7)$ & $-112.5(2)$ \\
\hline $\mathrm{C}(1)-\mathrm{C}(2)-\mathrm{C}(3)-\mathrm{N}(3)$ & $-136.66(19)$ \\
\hline $\mathrm{C}(7)-\mathrm{C}(2)-\mathrm{C}(3)-\mathrm{N}(3)$ & $-15.5(3)$ \\
\hline $\mathrm{C}(1)-\mathrm{C}(2)-\mathrm{C}(3)-\mathrm{C}(4)$ & $-13.6(2)$ \\
\hline $\mathrm{C}(7)-\mathrm{C}(2)-\mathrm{C}(3)-\mathrm{C}(4)$ & $107.5(2)$ \\
\hline $\mathrm{C}(1)-\mathrm{N}(1)-\mathrm{C}(4)-\mathrm{O}(2)$ & $178.0(2)$ \\
\hline $\mathrm{C}(5)-\mathrm{N}(1)-\mathrm{C}(4)-\mathrm{O}(2)$ & $-2.4(3)$ \\
\hline $\mathrm{C}(1)-\mathrm{N}(1)-\mathrm{C}(4)-\mathrm{C}(3)$ & $-3.1(2)$ \\
\hline $\mathrm{C}(5)-\mathrm{N}(1)-\mathrm{C}(4)-\mathrm{C}(3)$ & $176.50(18)$ \\
\hline $\mathrm{N}(3)-\mathrm{C}(3)-\mathrm{C}(4)-\mathrm{O}(2)$ & $-45.4(3)$ \\
\hline $\mathrm{C}(2)-\mathrm{C}(3)-\mathrm{C}(4)-\mathrm{O}(2)$ & $-170.3(2)$ \\
\hline $\mathrm{N}(3)-\mathrm{C}(3)-\mathrm{C}(4)-\mathrm{N}(1)$ & $135.76(19)$ \\
\hline $\mathrm{C}(2)-\mathrm{C}(3)-\mathrm{C}(4)-\mathrm{N}(1)$ & $10.9(2)$ \\
\hline $\mathrm{C}(4)-\mathrm{N}(1)-\mathrm{C}(5)-\mathrm{C}(6)$ & $101.9(3)$ \\
\hline $\mathrm{C}(1)-\mathrm{N}(1)-\mathrm{C}(5)-\mathrm{C}(6)$ & $-78.5(3)$ \\
\hline $\mathrm{C}(1)-\mathrm{C}(2)-\mathrm{C}(7)-\mathrm{C}(12)$ & $123.1(2)$ \\
\hline $\mathrm{C}(3)-\mathrm{C}(2)-\mathrm{C}(7)-\mathrm{C}(12)$ & $5.8(3)$ \\
\hline $\mathrm{C}(1)-\mathrm{C}(2)-\mathrm{C}(7)-\mathrm{C}(8)$ & $-59.0(3)$ \\
\hline $\mathrm{C}(3)-\mathrm{C}(2)-\mathrm{C}(7)-\mathrm{C}(8)$ & $-176.3(2)$ \\
\hline $\mathrm{C}(12)-\mathrm{C}(7)-\mathrm{C}(8)-\mathrm{C}(9)$ & $-0.9(4)$ \\
\hline $\mathrm{C}(2)-\mathrm{C}(7)-\mathrm{C}(8)-\mathrm{C}(9)$ & $-178.8(2)$ \\
\hline $\mathrm{C}(7)-\mathrm{C}(8)-\mathrm{C}(9)-\mathrm{C}(10)$ & $0.4(4)$ \\
\hline $\mathrm{C}(8)-\mathrm{C}(9)-\mathrm{C}(10)-\mathrm{C}(11)$ & $0.5(4)$ \\
\hline $\mathrm{C}(9)-\mathrm{C}(10)-\mathrm{C}(11)-\mathrm{C}(12)$ & $-0.8(4)$ \\
\hline $\mathrm{C}(8)-\mathrm{C}(7)-\mathrm{C}(12)-\mathrm{C}(11)$ & $0.6(3)$ \\
\hline$C(2)-C(7)-C(12)-C(11)$ & 178.41(19) \\
\hline $\mathrm{C}(8)-\mathrm{C}(7)-\mathrm{C}(12)-\mathrm{C}(13)$ & $-174.1(2)$ \\
\hline $\mathrm{C}(2)-\mathrm{C}(7)-\mathrm{C}(12)-\mathrm{C}(13)$ & $3.7(3)$ \\
\hline$C(10)-C(11)-C(12)-C(7)$ & $0.3(3)$ \\
\hline $\mathrm{C}(10)-\mathrm{C}(11)-\mathrm{C}(12)-\mathrm{C}(13)$ & $174.9(2)$ \\
\hline $\mathrm{C}(7)-\mathrm{C}(12)-\mathrm{C}(13)-\mathrm{N}(2)$ & $171.3(2)$ \\
\hline $\mathrm{C}(11)-\mathrm{C}(12)-\mathrm{C}(13)-\mathrm{N}(2)$ & $-3.4(3)$ \\
\hline $\mathrm{C}(7)-\mathrm{C}(12)-\mathrm{C}(13)-\mathrm{N}(3)$ & $-3.0(3)$ \\
\hline $\mathrm{C}(11)-\mathrm{C}(12)-\mathrm{C}(13)-\mathrm{N}(3)$ & $-177.68(19)$ \\
\hline $\mathrm{N}(3)-\mathrm{C}(13)-\mathrm{N}(2)-\mathrm{C}(14)$ & $0.2(2)$ \\
\hline
\end{tabular}




$\begin{array}{lc}\mathrm{C}(12)-\mathrm{C}(13)-\mathrm{N}(2)-\mathrm{C}(14) & -174.5(2) \\ \mathrm{N}(2)-\mathrm{C}(13)-\mathrm{N}(3)-\mathrm{C}(15) & -0.1(2) \\ \mathrm{C}(12)-\mathrm{C}(13)-\mathrm{N}(3)-\mathrm{C}(15) & 174.97(18) \\ \mathrm{N}(2)-\mathrm{C}(13)-\mathrm{N}(3)-\mathrm{C}(3) & 176.33(18) \\ \mathrm{C}(12)-\mathrm{C}(13)-\mathrm{N}(3)-\mathrm{C}(3) & -8.6(3) \\ \mathrm{C}(2)-\mathrm{C}(3)-\mathrm{N}(3)-\mathrm{C}(13) & 17.8(3) \\ \mathrm{C}(4)-\mathrm{C}(3)-\mathrm{N}(3)-\mathrm{C}(13) & -101.3(2) \\ \mathrm{C}(2)-\mathrm{C}(3)-\mathrm{N}(3)-\mathrm{C}(15) & -166.55(18) \\ \mathrm{C}(4)-\mathrm{C}(3)-\mathrm{N}(3)-\mathrm{C}(15) & 74.3(3) \\ \mathrm{C}(13)-\mathrm{N}(2)-\mathrm{C}(14)-\mathrm{C}(19) & 178.9(2) \\ \mathrm{C}(13)-\mathrm{N}(2)-\mathrm{C}(14)-\mathrm{C}(15) & -0.2(2) \\ \mathrm{C}(13)-\mathrm{N}(3)-\mathrm{C}(15)-\mathrm{C}(16) & -178.9(2) \\ \mathrm{C}(3)-\mathrm{N}(3)-\mathrm{C}(15)-\mathrm{C}(16) & 4.8(4) \\ \mathrm{C}(13)-\mathrm{N}(3)-\mathrm{C}(15)-\mathrm{C}(14) & 0.0(2) \\ \mathrm{C}(3)-\mathrm{N}(3)-\mathrm{C}(15)-\mathrm{C}(14) & -176.36(18) \\ \mathrm{N}(2)-\mathrm{C}(14)-\mathrm{C}(15)-\mathrm{N}(3) & 0.2(2) \\ \mathrm{C}(19)-\mathrm{C}(14)-\mathrm{C}(15)-\mathrm{N}(3) & -179.10(18) \\ \mathrm{N}(2)-\mathrm{C}(14)-\mathrm{C}(15)-\mathrm{C}(16) & 179.17(19) \\ \mathrm{C}(19)-\mathrm{C}(14)-\mathrm{C}(15)-\mathrm{C}(16) & -0.1(3) \\ \mathrm{N}(3)-\mathrm{C}(15)-\mathrm{C}(16)-\mathrm{C}(17) & 178.8(2) \\ \mathrm{C}(14)-\mathrm{C}(15)-\mathrm{C}(16)-\mathrm{C}(17) & 0.2(3) \\ \mathrm{C}(15)-\mathrm{C}(16)-\mathrm{C}(17)-\mathrm{C}(18) & -0.2(3) \\ \mathrm{C}(16)-\mathrm{C}(17)-\mathrm{C}(18)-\mathrm{C}(19) & 0.3(3) \\ \mathrm{C}(17)-\mathrm{C}(18)-\mathrm{C}(19)-\mathrm{C}(14) & -0.2(3) \\ \mathrm{N}(2)-\mathrm{C}(14)-\mathrm{C}(19)-\mathrm{C}(18) & -179.0(2) \\ \mathrm{C}(15)-\mathrm{C}(14)-\mathrm{C}(19)-\mathrm{C}(18) & 0.1(3) \\ & \\ & \end{array}$

Symmetry transformations used to generate equivalent atoms: 